\title{
Generation of Alkyl Radical through Direct Excitation of Boracene-based Alkylborate
}

Yukiya Sato $^{\dagger \perp}$, Kei Nakamura ${ }^{\dagger}$, Yuto Sumida ${ }^{\dagger *}$, Daisuke Hashizume ${ }^{\S}$, Takamitsu Hosoya ${ }^{\ddagger}$, and Hirohisa Ohmiya ${ }^{\dagger \# *}$

$\dagger$ Division of Pharmaceutical Sciences, Graduate School of Medical Sciences, Kanazawa University, Kakuma-machi, Kanazawa 920-1192, Japan

\$ Laboratory for Chemical Biology, RIKEN Center for Biosystems Dynamics Research (BDR), 6-7-3 Minatojima-minamimachi, Chuo-ku, Kobe 650-0047, Japan

$\S$ RIKEN Center for Emergent Matter Science (CEMS), 2-1 Hirosawa, Wako, Saitama 351-0198, Japan

" Laboratory of Chemical Bioscience, Institute of Biomaterials and Bioengineering, Tokyo Medical and Dental University (TMDU), 2-3-10 Kanda-Surugadai, Chiyoda-ku, Tokyo 101-0062, Japan \# JST, PRESTO, 4-1-8 Honcho, Kawaguchi, Saitama, 332-0012, Japan

*E-mail: Yuto Sumida: sumida@p.kanazawa-u.ac.jp

Takamitsu Hosoya: thosoya.cb@tmd.ac.jp

Hirohisa Ohmiya: ohmiya@p.kanazawa-u.ac.jp

\section{Table of Contents}

- Instrumentation and Chemicals $\quad$ S2

- Characterization Data for tert-butylborate 2a $\quad$ S3

- X-ray diffraction analysis for tert-butylborate 2a $\quad$ S4

- Stability Test of tert-butylborate 2 a $\quad$ S6

- UV-Vis Spectra of tert-butylborate 2a and Other Components $\quad$ S7

- Fluorescence Spectra of tert-butylborate 2a $\quad$ S8

- Cyclic Voltammetry of tert-butylborate 2a $\quad$ S8

- Estimation of the redox potential of $\mathbf{2 a} \quad$ S9

- Procedure for Radical Trap Experiment $\quad$ S10

- Dimerization via direct excitaion $\quad$ S11

- Procedure for Decyanoalkylation and a possible pathway $\quad$ S12

- Procedure for Giese Addition and a possible pathway $\quad$ S13

- Optimization of Ni-Catalyzed Alkylation $\quad$ S14

- Prorotocols for Ni-Catalyzed Alkylation $\quad$ S15

- Procedure for Ni-Catalyzed Three-Cmponent Coupling S16

- Characterization Data for Alkylation Products $\quad$ S17

- References $\quad$ S32

- NMR Spectra $\quad$ S33 


\section{- Instrumentation and Chemicals}

NMR spectra were recorded on a JNM-ECS400 or a JEOL $400 \mathrm{SS}$, operating at $400 \mathrm{MHz}$ for ${ }^{1} \mathrm{H}$ NMR, $100.5 \mathrm{MHz}$ for ${ }^{13} \mathrm{C} \mathrm{NMR}$, and, $127 \mathrm{MHz}$ for ${ }^{11} \mathrm{~B} \mathrm{NMR}$, and JNM-ECA600, operating at 600 $\mathrm{MHz}$ for ${ }^{1} \mathrm{H} \mathrm{NMR}$ and $150.9 \mathrm{MHz}$ for ${ }^{13} \mathrm{C}$ NMR. Chemical shift values for ${ }^{1} \mathrm{H},{ }^{13} \mathrm{C}$, and, ${ }^{11} \mathrm{~B}$ NMR are referenced to $\mathrm{Me}_{4} \mathrm{Si}$, the residual solvent resonances, and $\mathrm{BF}_{3} \cdot \mathrm{OEt}_{2}\left(\delta 0.0 \mathrm{ppm}\right.$ in $\left.\mathrm{CDCl}_{3}\right)$, respectively. Chemical shifts are reported in $\delta$ ppm. Mass spectra were obtained with a Thermo Scientific Exactive Plus Orbitrap or JMS-T100TD (DART). TLC analyses were performed on commercial glass plates bearing $0.25-\mathrm{mm}$ layer of Merck Silica gel $60 \mathrm{~F}_{254}$. Silica gel (Kanto Chemical Co., Silica gel 60 N, spherical, neutral) was used for column chromatography. IR spectra were measured with a Thermo Scientific iD7 ATR Accessory for the Thermo Scientific Nicolet iS5 FT-IR Spectrometer. Melting points were measured on a Yanaco MP-500D apparatus. CV measurements were recorded with a a CH Instruments: BAS Model 600E Series Electrochemical Analyzer. UV-Vis absorption spectra were recorded on a Shimadzu UV-1900. Fluorescence spectra were recorded on a Shimadzu RF-6000. Kessil PR160L $440 \mathrm{~nm}$ (highest blue and intensity setting) was used as a light source. Single-crystal X-ray diffraction data were collected on a Rigaku AFC-8 diffractometer equippted with Saturn70 CCD detector. The structures were solved by dual space method (SHELXT-2018) $)^{1}$ and refined by the full-matrix least-squares on $F^{2}$ (SHELXL-2018) ${ }^{2}$. CCDC 1871613 (compounds 2a) contain the supplementary crystallographic data for these structures. These data can be obtained free of charge from the Cambridge Crystallographic Data Centre (CCDC) via www.ccdc.cam.ac.uk/data_request/cif.

All reactions were carried out under nitrogen or argon atmosphere. Materials were obtained from commercial suppliers stored under nitrogen, and used as received or prepared according to standard procedures unless otherwise noted. 8,9-Dioxa-8a-borabenzo[fg]tetracene (boracene) (1) was prepared by the reported procedure ${ }^{3,4}$ or purchased by Sumika Technoservice Corporation, and used as received. Dimethylacetoamide was purchased from Nacalai Tesque Inc. Dehydrated tetrahydrofuran, acetonitrile, and methanol were purchased from FUJIFILM Wako Pure Chemical Co. tertButyllithium (in $n$-pentane, $1.6 \mathrm{~mol} / \mathrm{L}$ ) was purchased from Kanto Chemical Co. Inc. Ni(acac)2, $\mathrm{Ni}(\mathrm{TMHD})_{2}, \mathrm{NiBr} \cdot$ diglyme, 4,4'-di-tert-butylbiphenyl, lithium wire (in mineral oil, diam. $3.2 \mathrm{~mm}$, 99.9\% trace metals basis), and dtbpy, were purchased from Sigma-Aldrich. THF- $d_{8}$ was purchased from Eurisotop. 2-Chloro-2-methylpropane- $d_{9}(99.3 \% \mathrm{D})$ was purchased from CDN isotopes. 


\section{tert-Butylborate 2a}
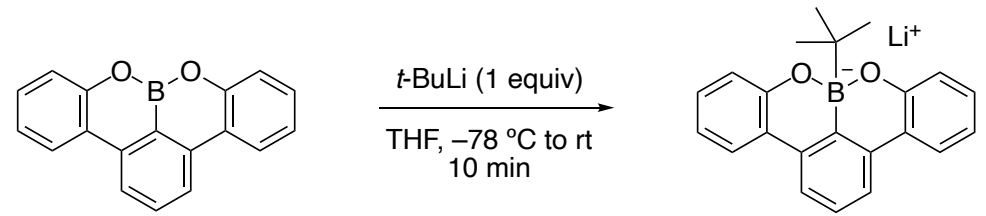

To a suspension of boracene $(270 \mathrm{mg}, 1.00 \mathrm{mmol})$ in THF $(10 \mathrm{~mL})$ was added $t$-butyllithium $(1.6$ $\mathrm{M}$ in $n$-pentane, $0.6 \mathrm{~mL}, 1.0 \mathrm{mmol}$ ) at $-78^{\circ} \mathrm{C}$. After warming to room temperature, the mixture was stirred for $10 \mathrm{~min}$ at the same temperature. The mixture was concentrated under reduced pressure to give $\mathbf{2 a}(475 \mathrm{mg}, 993 \mu \mathrm{mol}, 99.3 \%)$ as a colorless solid.

The molecular weight of $\mathbf{2 a}$ was calculated as $[t-\mathrm{Bu}$ (boracene) $] \mathrm{Li} \cdot 2 \mathrm{THF}(\mathrm{MW}: 478.36)$ determined from ${ }^{1} \mathrm{H}$ NMR and the X-ray structure, for the use of the experiments.

M.p. $163^{\circ} \mathrm{C}$ (decomp.).

IR (ZnSe, $\left.\mathrm{cm}^{-1}\right): 731,754,899,918,947,1042,1130,1234,1290,1406,1447,1578,2843,2884$, 2920; The number of coordinated THF was determined from the integral value of ${ }^{1} \mathrm{H}$ NMR.

${ }^{1}$ H NMR (400 MHz, $\mathrm{CD}_{2} \mathrm{Cl}_{2}$ ) $\delta 0.46$ (s, 9H), 1.61-1.72 (m, THF), 3.54-3.65 (m, THF), 6.80-6.89 (m, 4H), $7.10(\mathrm{ddd}, J=1.6,8.0,8.0 \mathrm{~Hz}, 2 \mathrm{H}), 7.31(\mathrm{t}, J=8.0 \mathrm{~Hz}, 1 \mathrm{H}), 7.56(\mathrm{~d}, J=8.0 \mathrm{~Hz}, 2 \mathrm{H}), 7.76$ $(\mathrm{dd}, J=1.6,8.0 \mathrm{~Hz}, 2 \mathrm{H})$

${ }^{13} \mathrm{C}$ NMR (100 MHz, $\mathrm{CD}_{2} \mathrm{Cl}_{2}$ ) $\delta 25.6$ (THF), 27.3 (3C), 68.6 (THF), 119.0 (2C), 119.5 (2C), 120.4 (2C), 124.0 (2C), 127.1, 128.4 (2C), 128.6 (2C), 135.7 (2C), 156.7 (2C). The signals for the carbons attached to the boron atom were not observed.

${ }^{11}$ B NMR (127 MHz, $\left.\mathrm{CD}_{2} \mathrm{Cl}_{2}\right) \delta 7.49$ (br).

HRMS $\left(\mathrm{ESI}^{-} \mathrm{m} / z\right)$ : [M-( $\left.\left.\mathrm{Li}+2 \mathrm{THF}\right)\right]^{-}$calcd for $\mathrm{C}_{22} \mathrm{H}_{20} \mathrm{BO}_{2}^{-}, 327.1562$; found, 327.1564 . 

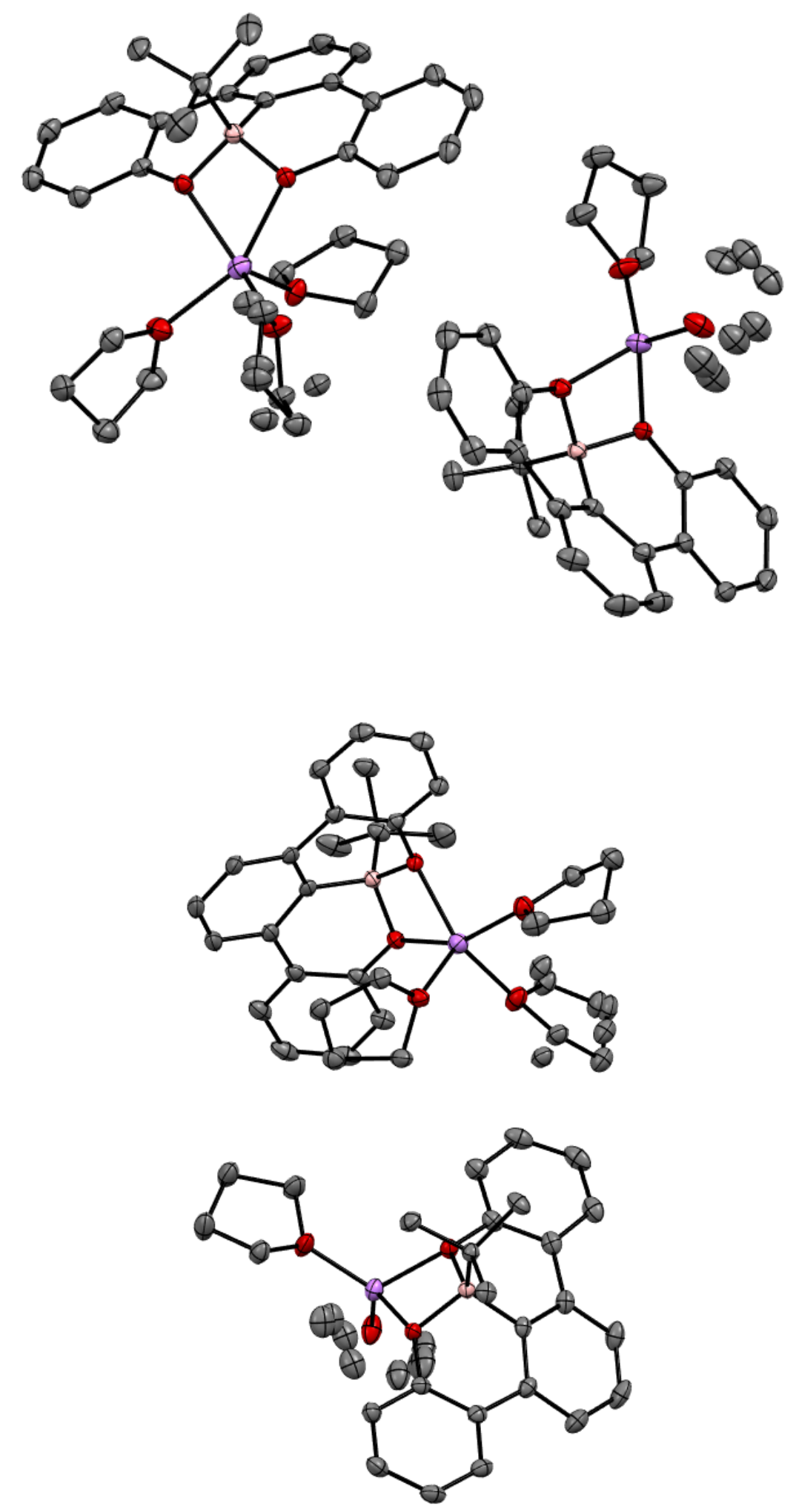

Figure S1. ORTEP diagram of 2a. Ellipsoids are drawn at 50\% probability. A single crystal used for the analysis was obtained by recrystallization from $n$-hexane and tetrahydrofuran. Hydrogen atoms are omitted for clarity. 
Table S1. Crystal data and structure refinement for $\mathbf{2 a}$

Identification code

Empirical formula moiety

Empirical formula sum

Formula weight sum

Temperature

Wavelength

Crystal system

Space group

Unit cell dimensions

\section{$Z$}

$D_{\mathrm{X}}$

$\mu$

$F(000)$

Crystal size

Theta range for data collection

Index ranges

Reflections collected

Independent reflections

Completeness

Absorption correction

Max. and min. transmission

Refinement method

Data / restraints / parameters

Goodness-of-fit on $F^{2}$

Final $R$ indices $[I>2 \sigma(I)]$

$R$ indices (all data)

Largest diff. peak and hole compound 2a

$\mathrm{C}_{34} \mathrm{H}_{44} \mathrm{BLiO}_{5}, \mathrm{C}_{30} \mathrm{H}_{36} \mathrm{BLiO}_{4}$

$\mathrm{C}_{64} \mathrm{H}_{80} \mathrm{~B}_{2} \mathrm{Li}_{2} \mathrm{O}_{9}$

1028.78

$90 \mathrm{~K}$

$0.71073 \AA$

Monoclinic

$P 2{ }_{1} / n$

$a=10.82397(12) \AA$

$b=16.16979(18) \AA$

$c=32.6888(4) \AA$

$V=2589.27 \AA^{3}$

4

$1.203 \mathrm{Mg} / \mathrm{m}^{3}$

$0.077 \mathrm{~mm}^{-1}$

2208

$0.152 \times 0.117 \times 0.112 \mathrm{~mm}^{3}$

1.906 to $32.683^{\circ}$

$-14 \leq h \leq 14,-20 \leq k \leq 20,-42 \leq l \leq 42$

224284

$13012\left[R_{\text {int }}=0.0573\right]$

$100 \%$

multi-scan

1.00000 and 0.92851

Full-matrix least-squares on $F^{2}$

13012 / 26 / 726

1.029

$R(F)=0.0456, w R\left(F^{2}\right)=0.1082$

$R(F)=0.0570, w R\left(F^{2}\right)=0.1152$

0.925 and -0.310 e $\AA^{-3}$ 
- Stability Test of tert-butylborate 2a a

The stability of $\mathbf{2 a},(2.5 \mathrm{mg})$ was examined by monitoring the decrease of the complex by ${ }^{1} \mathrm{H}$ NMR in $\mathrm{CD}_{2} \mathrm{Cl}_{2}(500 \mu \mathrm{L})$ at room temperature (Figures $\mathrm{S} 2$ ).

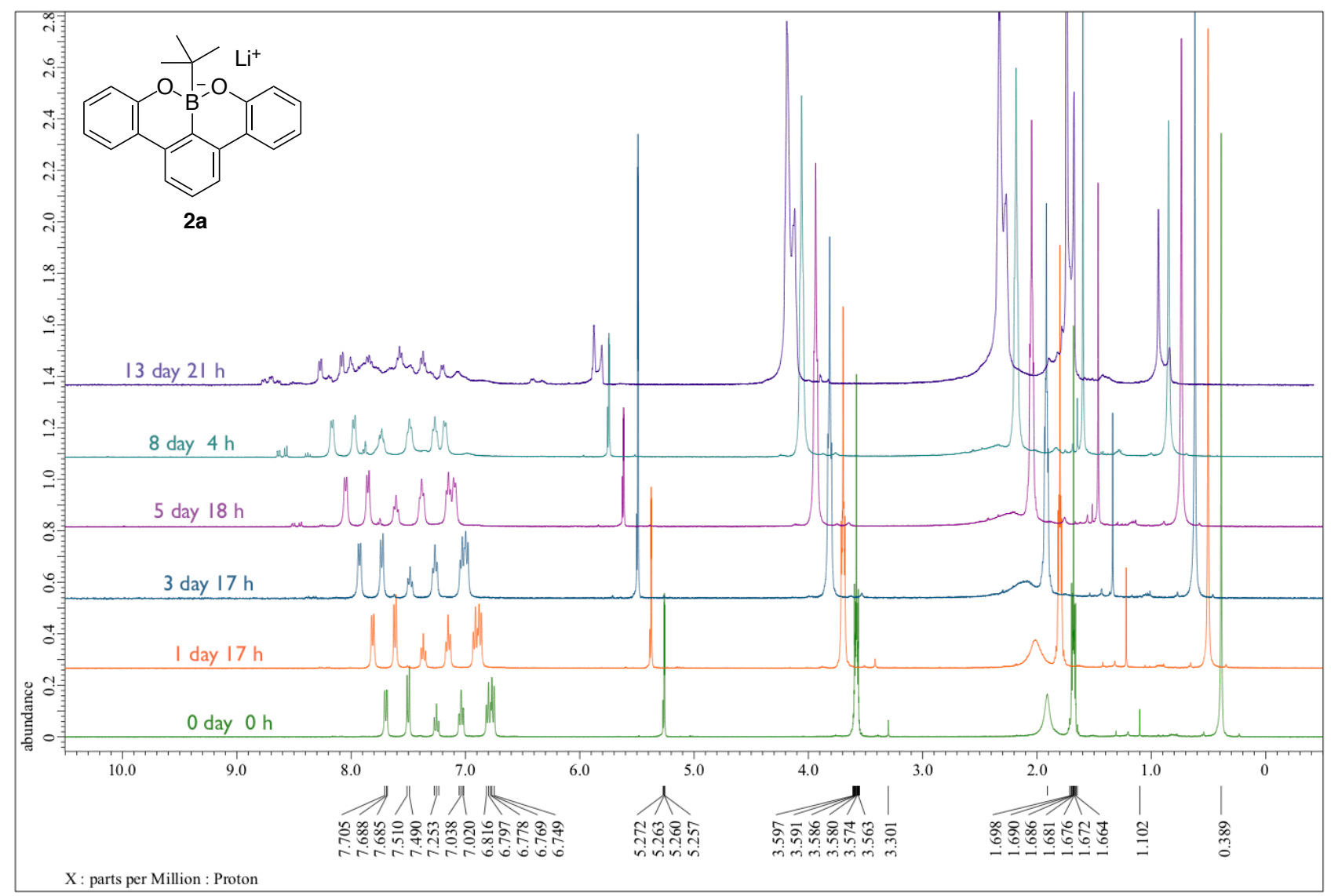

Figure S2. Monitoring of time-dependent degradation of 2a. 
- UV-Vis Spectra of tert-butylborate 2a and Other Components

$\mathrm{UV}$-Vis absorption spectra of boracene $\mathbf{1}$ and tert-butylborate $\mathbf{2 a}$ were measured with $20 \mu \mathrm{M}$ or 0.1 M DMA solution.
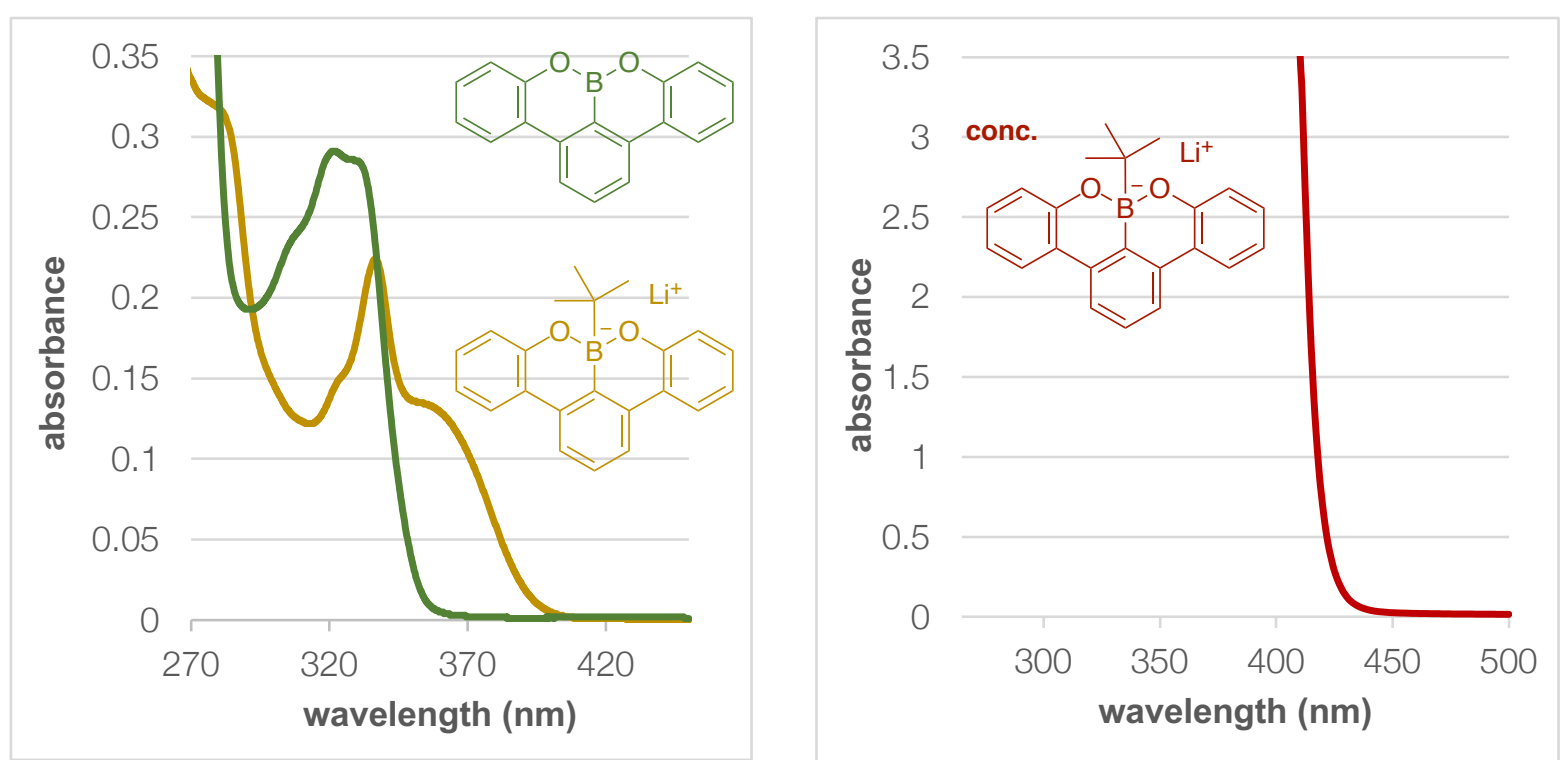

Figure S3. UV-Vis absorption spectra of $\mathbf{1}$ and 2a. Green line: boracene $\mathbf{1}$ (20 $\mu \mathrm{M}$ in DMA). Yellow line tert-butylborate 2a (100 $\mu \mathrm{M}$ in DMA). Red line: tert-butylborate 2a (0.1 M in DMA).

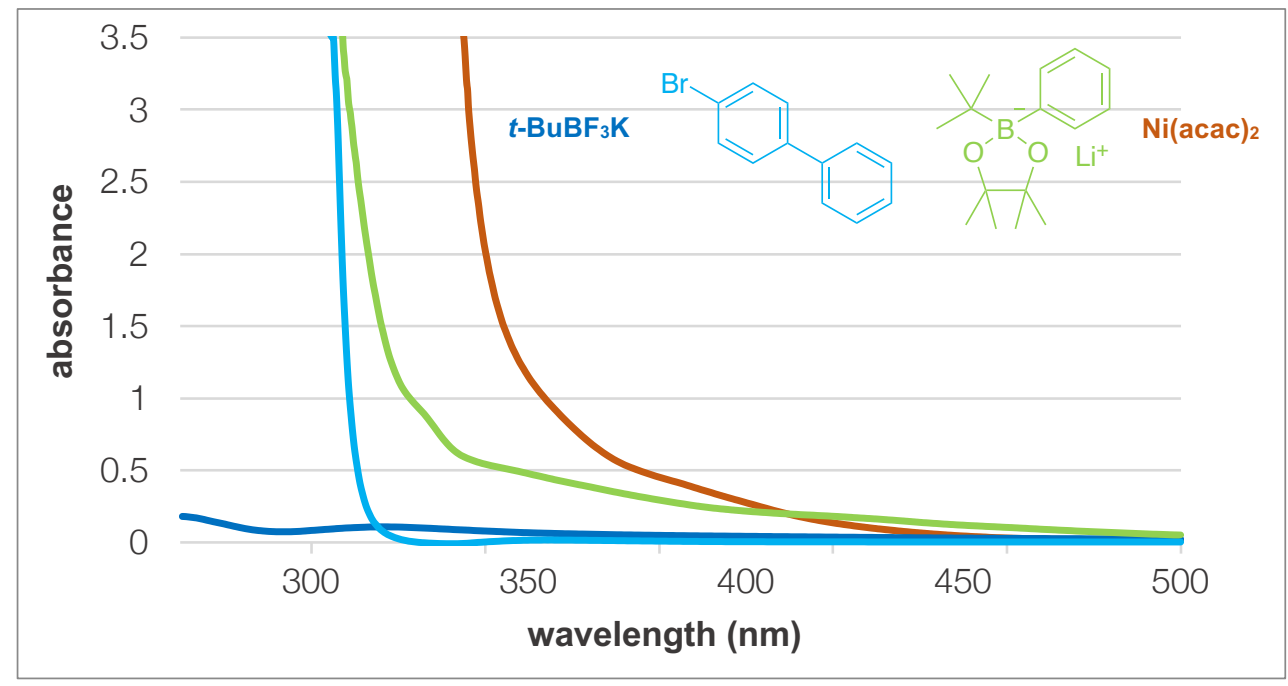

Figure S4. UV-Vis absorption spectra of $\mathrm{Ni}(\mathrm{acac})_{2},[t-\mathrm{Bu}(\mathrm{Ph}) \mathrm{Bpin}] \mathrm{Li}$, 4-bromobiphenyl, and $t$ $\mathrm{BuBF}_{3} \mathrm{~K}$. Orange line: $\mathrm{Ni}(\mathrm{acac})_{2}(0.1 \mathrm{M}$ in DMA). Light green line: $t$-Bu(Ph)Bpin]Li $(0.1 \mathrm{M}$ in DMA). Light blue line: 4-bromobiphenyl (0.1 M in DMA). Blue line: $t$-BuBF ${ }_{3} \mathrm{~K}$. (0.1 M in DMA). 
- Fluorescence Spectra of tert-butylborate 2a

Fluorescence spectra of boracene 1 and tert-butylborate $\mathbf{2 a}$ were measured with $20 \mu \mathrm{M}$ DMA solution.
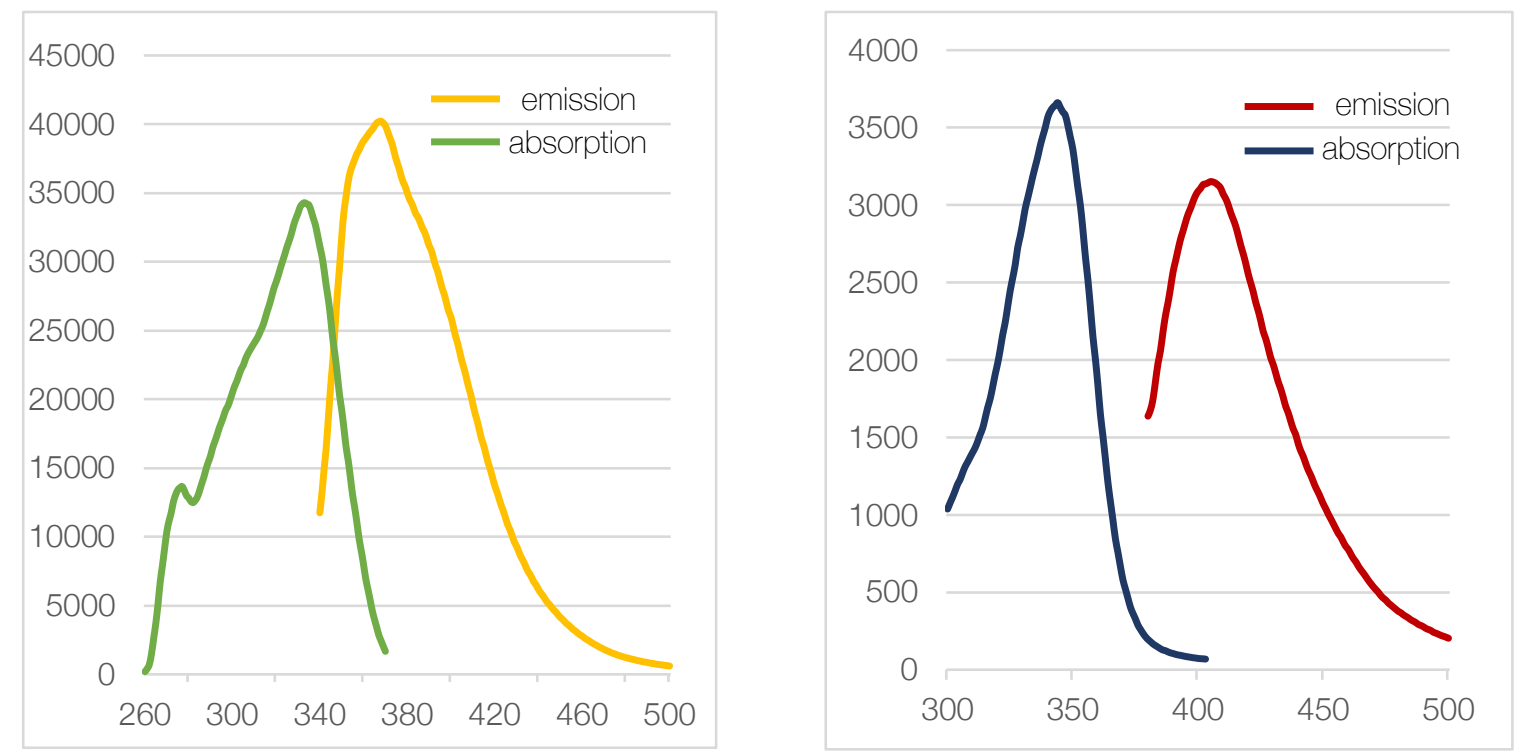

Figure S5. Fluorescence spectra of boracene $\mathbf{1}$ and $t$-butylborate $\mathbf{2 a}(20 \mu \mathrm{M}$ in DMA). Green line: absorption spectra of $\mathbf{1}$, yellow line: emission spectra of $\mathbf{1}$ (excitation at $330 \mathrm{~nm}$ ). Blue line: absorption spectra of 2a, red line: emission spectra of $\mathbf{2 a}$ (excitation at $370 \mathrm{~nm}$ ).

\section{- Cyclic Voltammetry of tert-butylborate 2a}

CV voltammogram of alkylborate $2 \mathbf{a}$ was recorded in dry, degassed acetonitrile solution with 0.1 $\mathrm{M}$ of tetrabutylammonium hexafluorophosphate $\left(\left[\mathrm{N}(n \mathrm{Bu})_{4}\right] \mathrm{PF}_{6}\right)$ as a supporting electrolyte. Measurements used a glassy carbon working electrode (area $=0.07 \mathrm{~cm}^{2}$ ), an saturated calomel reference electrode (SCE), and a Pt wire counter electrode. The concentration of the sample solution was fixed at $2 \mathrm{mM}$ and the sweep rates were set to $100 \mathrm{mV} / \mathrm{s}$.

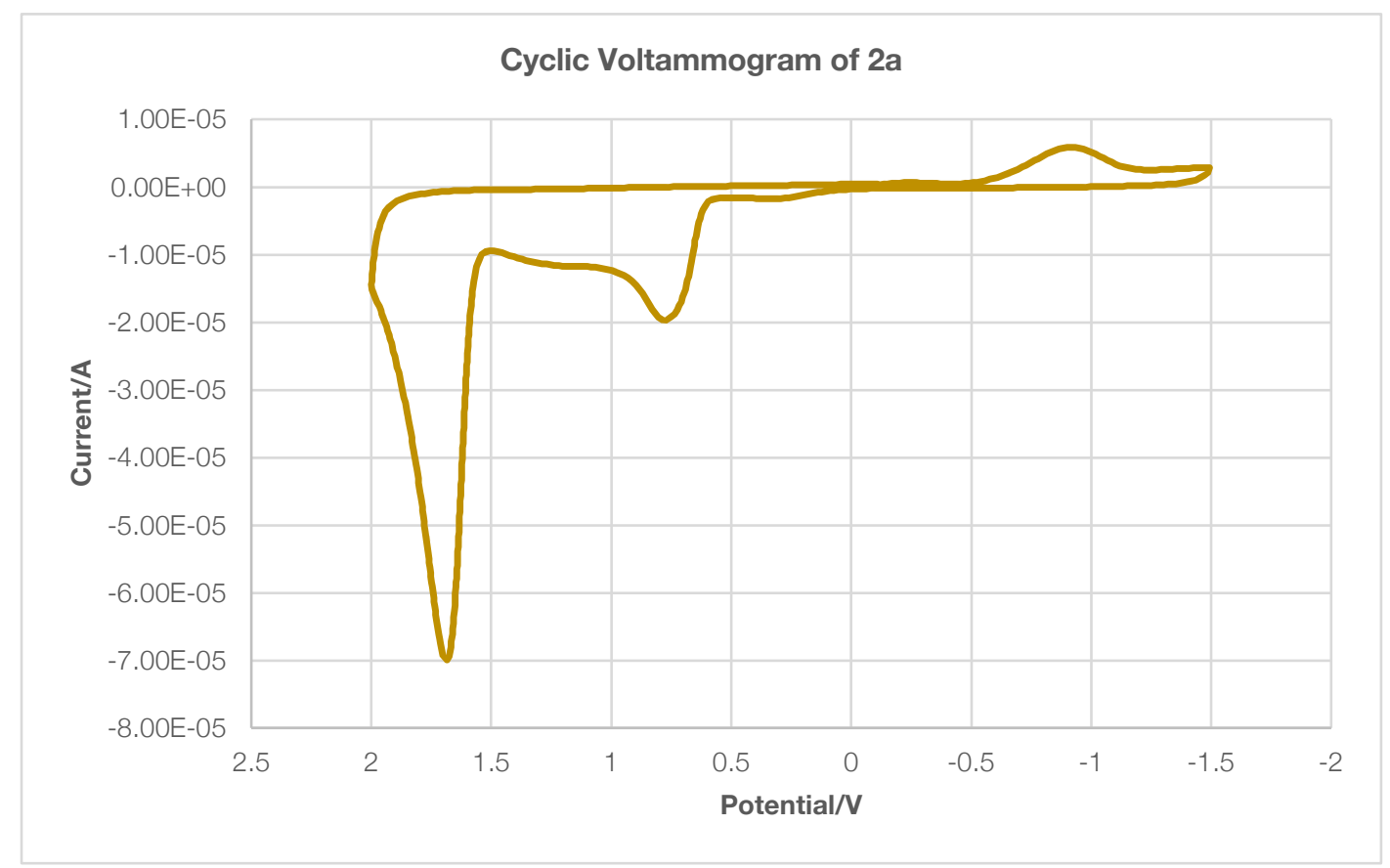

Figure S6. Cyclic voltammogram of $2 \mathbf{a}$. 


\section{- Estimation of the redox potential of 2a}

Based on the data obtained from the UV/Vis, fluorescence spectra and the cyclic voltammetry, RehmWeller equation described below afforded the apploximated redox potential value of the photoexcited tert-butylborate $\mathbf{2 a}$ *.

Rehm-Weller equation:

$$
\begin{gathered}
\mathrm{E}\left(\mathbf{2} \mathbf{a}^{+\bullet} / \mathbf{2} \mathbf{a}^{*}\right)=\mathrm{E}\left(\mathbf{2} \mathbf{a}^{+\bullet} / \mathbf{2} \mathbf{a}\right)-E_{00}(\mathbf{2} \mathbf{a} * / \mathbf{2 a}) \\
\mathrm{E}\left(\mathbf{2} \mathbf{a}^{+\bullet} / \mathbf{2} \mathbf{a}^{*}\right)=0.78-3.02=-\mathbf{2 . 2 4} \mathbf{V} \text { vs. SCE. }
\end{gathered}
$$

According to cyclic voltammetry, the electrochemical oxidation of tert-butylborate $\mathbf{2 a}$ is an irreversible step. Therefore, we used an anodic half peak potential $\mathrm{E}_{\mathrm{pa} / 2}(=+0.78 \mathrm{~V}$ vs. SCE $)$ as $\mathrm{E}$ $\left(\mathbf{2} \mathbf{a}^{+\bullet} / \mathbf{2 a}\right)$. The excited state energy of tert-butylborate $\mathbf{2 a}, \mathrm{E}_{00}(\mathbf{2} \mathbf{a} * / \mathbf{2 a})$, could be estimated from the tail of the wavelength tail of the UV/Vis absorption and excitation spectra (410 nm, Figure S3 and 5), which converted to $3.02 \mathrm{eV}$ and used as $\mathrm{E}_{00}\left(\mathbf{2 a}^{+\bullet} / \mathbf{2} \mathbf{a}^{*}\right)$. 
- Procedure for Radical Trap Experiment a<smiles>CC(C)(C)B1Oc2ccccc2-c2cccc3c2B1Oc1ccccc1-3</smiles>

2a<smiles>CC1(C)CCCC(C)(C)N1[O]</smiles>

TEMPO

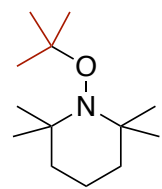

3a

In a glovebox, to an oven-dried vial with a stirring bar was added tert-butylborate $\mathbf{2 a}(95.7 \mathrm{mg}, 0.20$ mmol), 2,2,6,6-tetramethylpiperidine 1-oxyl, TEMPO (15.6 mg, $0.20 \mathrm{mmol}$ ) and MeCN (400 $\mu \mathrm{l})$. After sealing the vial with parafilm, the reaction was placed in EvoluChem photoreactor (PhotoRedOx Duo) equiped with a 45W blue LED (Figure S7). After stirred for $14 \mathrm{~h}$, the reaction was quenched with a short plug of silica gel using ethyl acetate. After volatiles were removed under reduced pressure, purification by flash column chromatography on silica gel gave the 3a (27.5 mg, $64 \%$ isolated yield) with the recovery of boracene 1 as a colorless solid.
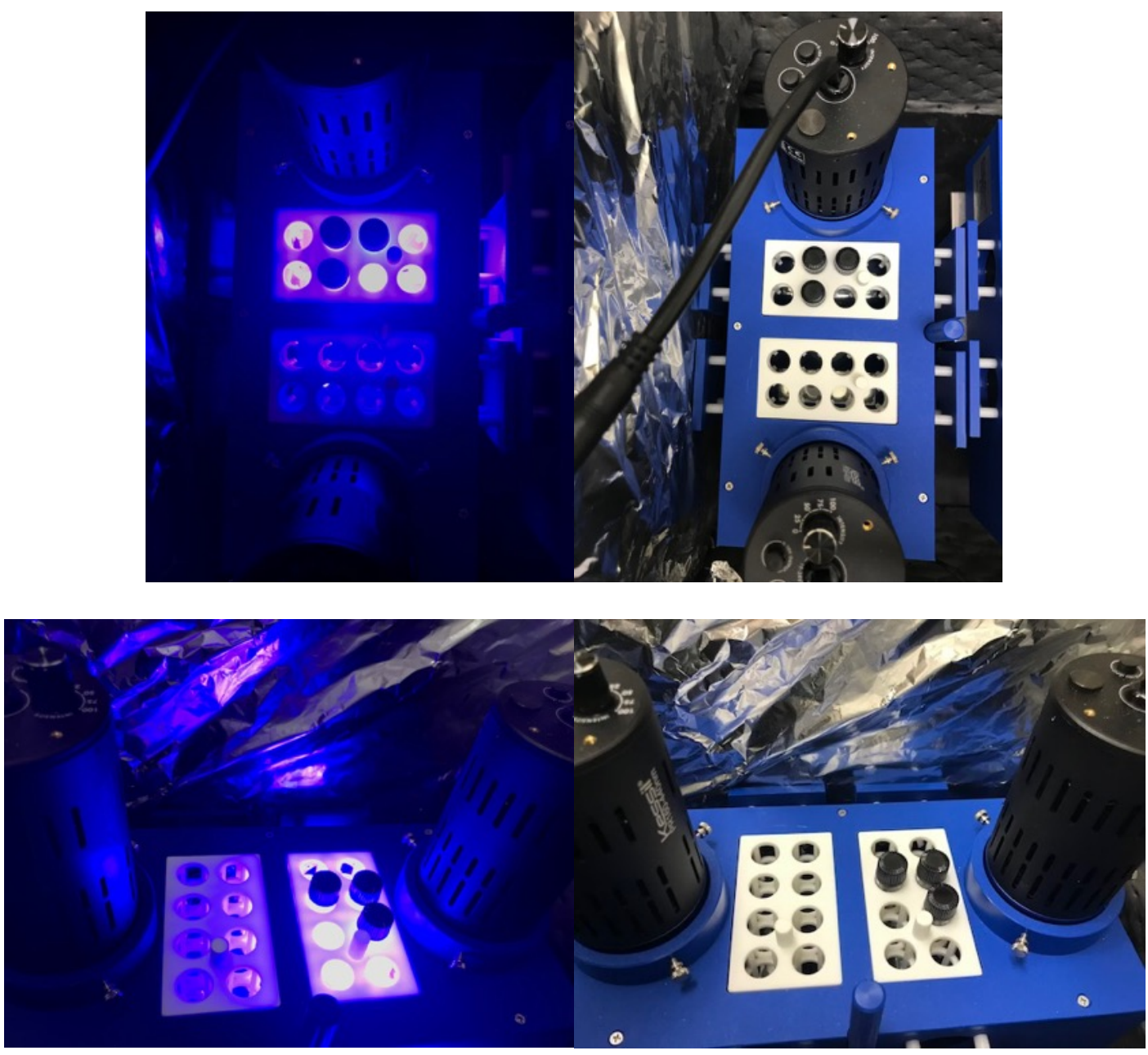

Figure S7. Light set up 
- Dimerization via direct excitaion<smiles>C1=CC2=C3B(Oc4ccccc43)c3ccccc3C2=C1</smiles>

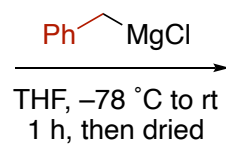

$1 \mathrm{~h}$, then dried

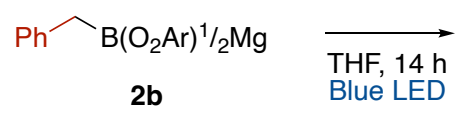<smiles>c1ccc(Pc2ccccc2)cc1</smiles>

To a suspension of boracene 1 ( $54 \mathrm{mg}, 0.2 \mathrm{mmol})$ in THF (200 $\mu \mathrm{l})$ was added benzylmagnesium chloride solution $(300 \mu \mathrm{l}, 0.22 \mathrm{mmol})$ in THF $(0.75 \mathrm{M})$ prepared by conventional method at $-78{ }^{\circ} \mathrm{C}$ and then allowed to warm up to room temperature. After stirred for $2 \mathrm{~h}$, passed through a short plug of $\mathrm{N}-\mathrm{H}$ silica gel to give benzylborate $\mathbf{2 b}$ in THF solution. the reaction was placed in EvoluChem photoreactor (PhotoRedOx Duo) equiped with a 45W blue LED (Figure S7). After stirred for $14 \mathrm{~h}$, the reaction was quenched with a short plug of silica gel using ethylacetate. After volatiles were removed under reduced pressure, the residue was dissolved in $\mathrm{CDCl}_{3}(500 \mu \mathrm{L})$ and added 1,1,2,2tetrachloroethane $(11 \mu \mathrm{L}, 10.5 \mu \mathrm{mol})$ as an internal standard. ${ }^{1} \mathrm{H}$ NMR (400 MHz) yield (quantitative) was obtained by comparing the relative value of integration for the doublet peak observed at 2.92 ppm of bibenzyl with that of 1,1,2,2-tetrachloroethane observed at 5.95 ppm (Figure S8).

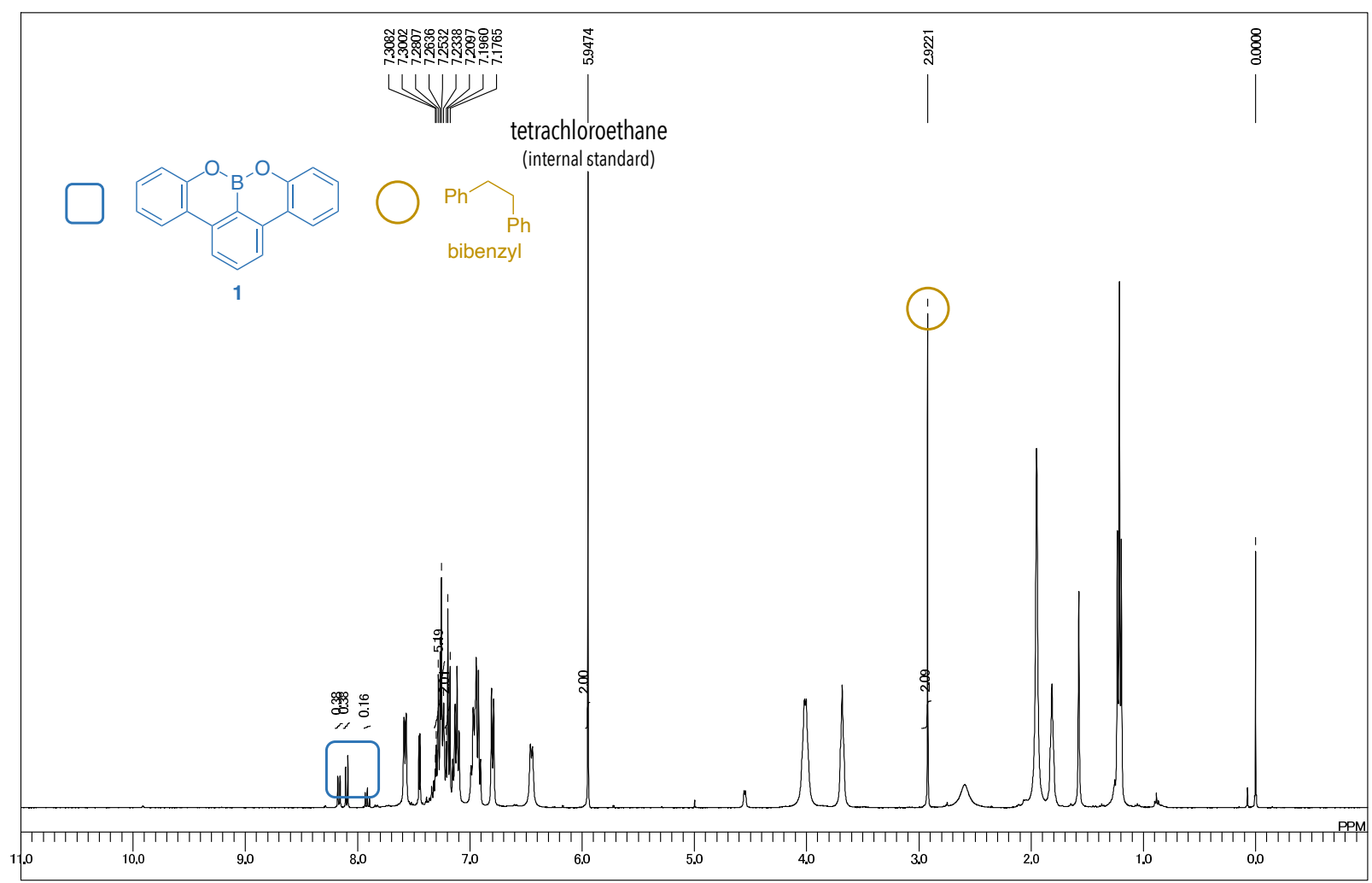

Figure S8. ${ }^{1} \mathrm{H}$ NMR analysis 


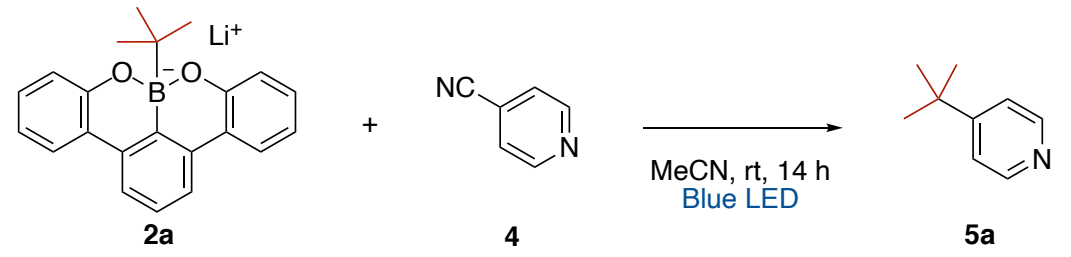

In a glovebox, to an oven-dried vial with a stirring bar was added tert-butylborate $2 \mathrm{a}(71.8 \mathrm{mg}$, $0.15 \mathrm{mmol})$, 4-cyanopyridine (4) $(10.4 \mathrm{mg}, 0.1 \mathrm{mmol})$ and $\mathrm{MeCN}(100 \mu \mathrm{l})$. After sealing the vial with parafilm, the reaction was placed in EvoluChem photoreactor (PhotoRedOx Duo) equiped with a 45W blue LED (Figure S7). After stirred for $14 \mathrm{~h}$, the reaction was quenched with a short plug of silica gel using ethylacetate. After volatiles were removed under reduced pressure, the residue was dissolved in $\mathrm{CDCl}_{3}(500 \mu \mathrm{L})$ and added 1,1,2,2-tetrachloroethane $(11 \mu \mathrm{L}, 10.5 \mu \mathrm{mol})$ as an internal standard. ${ }^{1} \mathrm{H}$ NMR $(400 \mathrm{MHz})$ yield $(80 \%)$ was obtained by comparing the relative value of integration for the doublet peak observed at $8.40 \mathrm{ppm}$ of $\mathbf{5 a}$ with that of 1,1,2,2-tetrachloroethane observed at 5.96 ppm (Figure S9). The generation of 5a was also confirmed by observation of the corresponding molecular ion peak with GC-MS analysis.

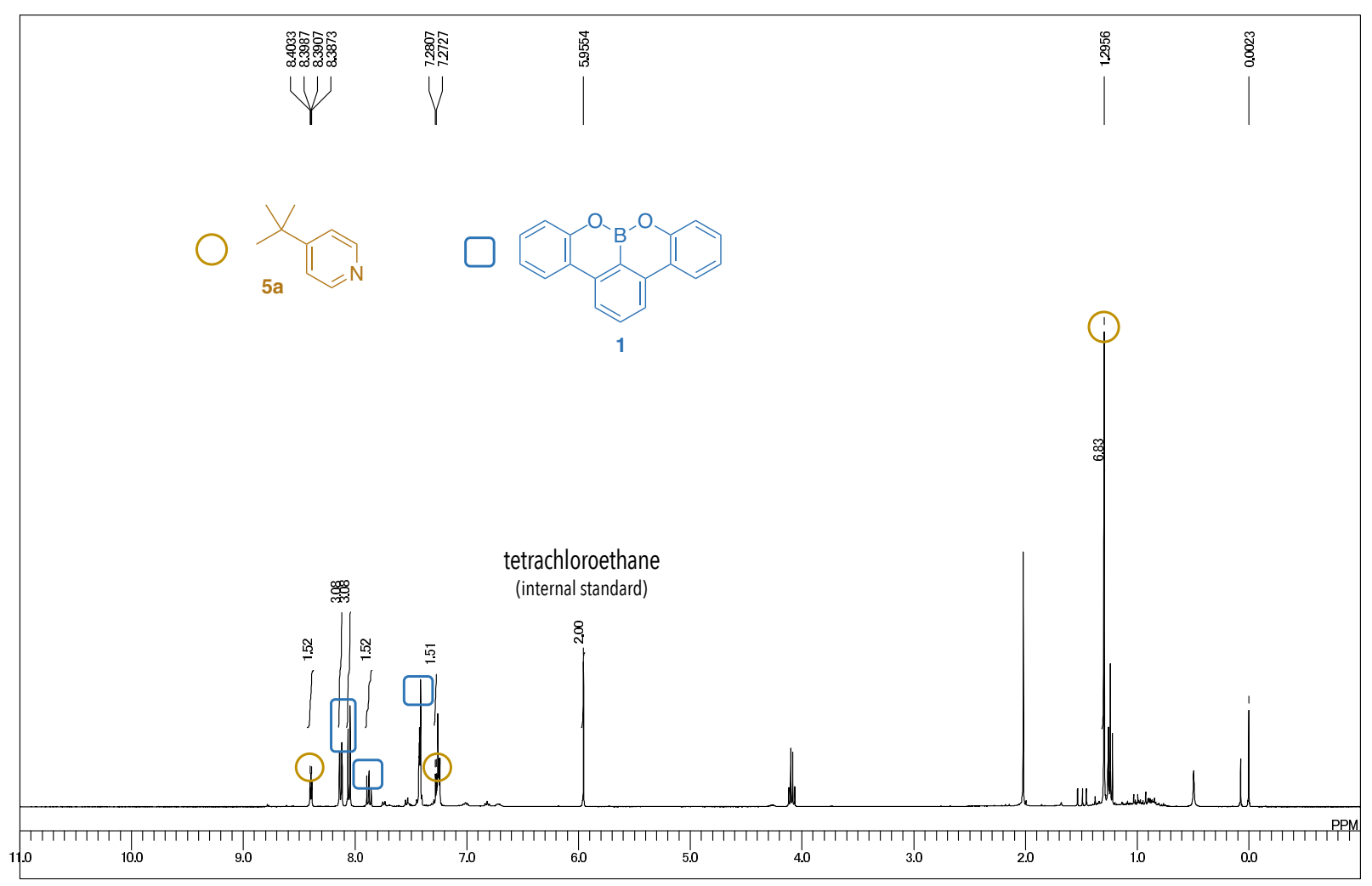

Figure S9. ${ }^{1} \mathrm{H}$ NMR analysis 
- Possible pathway for decyanoalkylation of 4-cyanopyridine

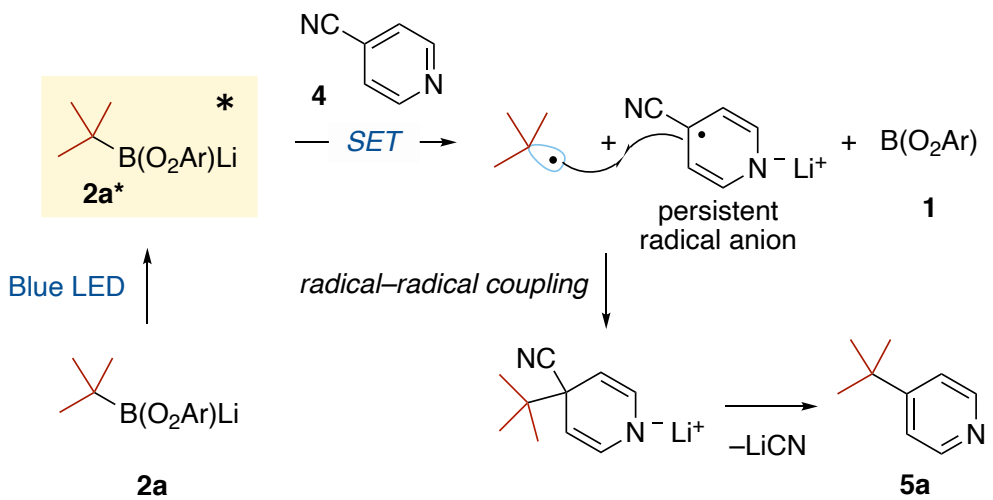

\section{- Procedure for Giese Addition}

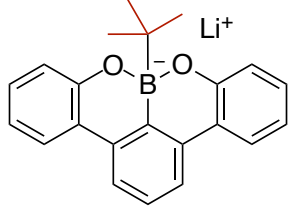

2a

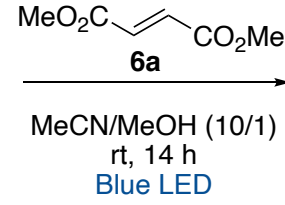

Blue LED

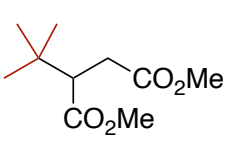

7 aa

In a glovebox, to an oven-dried vial with a stirring bar was added tert-butylborate $\mathbf{2 a}(71.8 \mathrm{mg}$, $0.15 \mathrm{mmol})$, dimethylfumarate $6 \mathbf{a}(14.4 \mathrm{mg}, 0.1 \mathrm{mmol})$ and $\mathrm{MeCN} / \mathrm{MeOH}(9 / 1,100 \mu \mathrm{l})$. After sealing the vial with parafilm, the reaction was placed in EvoluChem photoreactor (PhotoRedOx Duo) equiped with a $45 \mathrm{~W}$ blue LED (Figure S7). After stirred for $14 \mathrm{~h}$, the reaction was quenched with a short plug of silica gel using ethyl acetate. After volatiles were removed under reduced pressure, purification by flash column chromatography on silica gel gave the 7aa (14.0 $\mathrm{mg}, 69 \%$ isolated yield) with the recovery of boracene $\mathbf{1}$ as a colorless solid.

\section{- Possible pathway for Giese addition}

In addition to that simple photo-irradiation can trigger the generation of alkyl radical, since other Michael acceptor, such as acrylate, can be applied (cf. Table 2 in Manuscript), 1,4-addition of an alkyl radical would be reasonable.

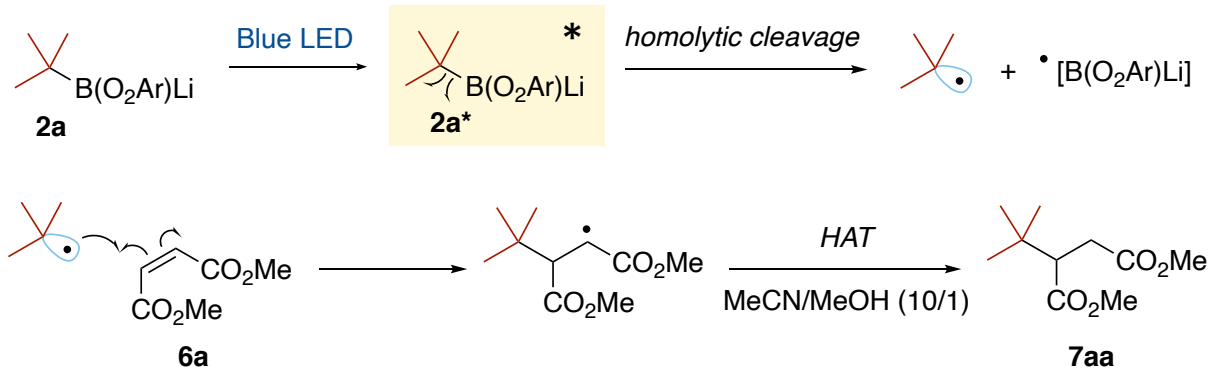




\section{- Optimization of Ni-Catalyzed Alkylation}

Table S2. Optimization of reaction conditions.

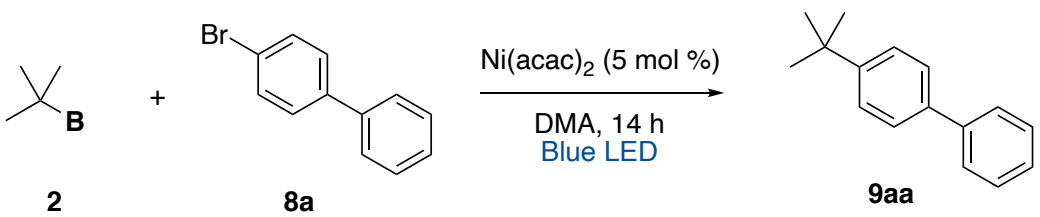

\begin{tabular}{|c|c|c|}
\hline Entry & deviation from standard conditions $\mathrm{s}^{\mathrm{a}}$ & Yield (\%) \\
\hline 1 & none (Scheme 1) & 74 \\
\hline 2 & $390 \mathrm{~nm}$ light instead of $440 \mathrm{~nm}$ & $40^{\mathrm{b}}$ \\
\hline 3 & no light & 0 \\
\hline 4 & tert-BuBF $\mathbf{B}_{3} \mathbf{K}$ instead of $\mathbf{2 a}$ & 0 \\
\hline 5 & tert-BuBF $\mathbf{B}_{3} \mathbf{K}$ instead of $2 \mathrm{a}^{\mathrm{c}}$ & 0 \\
\hline 6 & {$\left[\right.$ tert-Bu(Ph)Bpin] $\mathbf{L i}^{\mathrm{d}}$, instead of $\mathbf{2 a}$} & 0 \\
\hline 7 & $\mathrm{Ni}(\operatorname{cod})_{2}$ instead of $\mathrm{Ni}(\mathrm{acac})_{2}$ & trace \\
\hline 8 & $\mathrm{Ni}(\operatorname{cod})_{2}$, acetylacetone ${ }^{\mathrm{e}}, \mathbf{K O t B u}^{\mathrm{e}}$, instead of $\mathrm{Ni}(\mathrm{acac})_{2}$ & $59^{\mathrm{b}}$ \\
\hline 9 & $\mathbf{N i}(\text { HFPD })_{2}{ }^{\mathrm{f}}$ instead of $\mathrm{Ni}(\mathrm{acac})_{2}$ & $9^{b}$ \\
\hline 10 & $\mathbf{N i ( T M H D})_{2}{ }^{\mathrm{g}}$ instead of $\mathrm{Ni}(\mathrm{acac})_{2}$ & $74^{\mathrm{b}}$ \\
\hline 11 & $\mathbf{F e}(\mathbf{a c a c})_{2}$ instead of $\mathrm{Ni}(\mathrm{acac})_{2}$ & 0 \\
\hline 12 & $\mathbf{P d}(\mathbf{a c a c})_{2}$ instead of $\mathrm{Ni}(\mathrm{acac})_{2}$ & 0 \\
\hline 13 & $\mathbf{C o}(\mathbf{a c a c})_{2}$ instead of $\mathrm{Ni}(\mathrm{acac})_{2}$ & 0 \\
\hline 14 & $\mathbf{C u}(\mathbf{a c a c})_{2}$ instead of $\mathrm{Ni}(\mathrm{acac})_{2}$ & 0 \\
\hline
\end{tabular}

${ }^{a}$ Reaction was carried out with $2 \mathbf{a}(0.12 \mathrm{mmol}), \mathbf{8 a}(0.1 \mathrm{mmol})$, catalyst $(5.0 \mu \mathrm{mol})$ in DMA $(1 \mathrm{~mL})$ under blue LED (440 $\mathrm{nm}$ ) irradiation for 14 h. ${ }^{b} \mathrm{H}$ NMR yield based on $\mathbf{8 a} .{ }^{c} 10 \mathrm{~mol} \%$ of Ni(TMHD), $\mathrm{ZnBr}_{2}$, and, 1.0 equiv of $\mathrm{K}_{2} \mathrm{HPO}_{4}$ were used. ${ }^{d}$ The borate was prepared from precomplexation of tert-BuBpin with PhLi. ${ }^{e} 10$ mol $\%$ was used. ${ }^{f} \mathrm{HFPD}=$ 1,1,1,5,5,5-hexafluoropentane-2,4-dione. ${ }^{g}$ TMHD = 2,2,6,6-tetramethyl-5-heptanedione. 


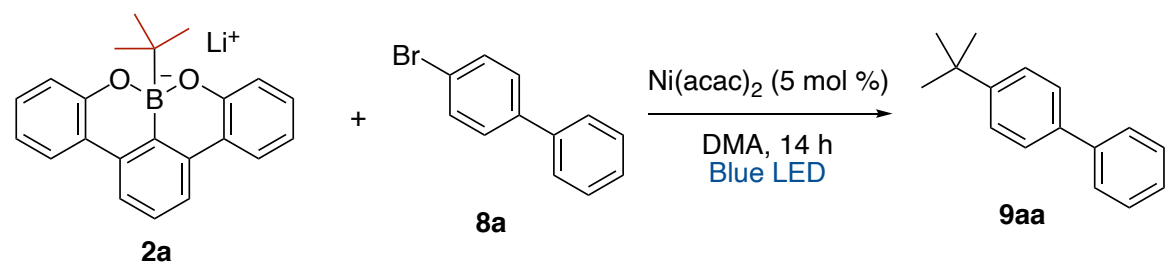

Method A (synthesis of 9aa as a representative): In a glovebox, to an oven-dried vial with a stirring bar was added $\mathrm{Ni}(\mathrm{acac})_{2}(2.6 \mathrm{mg}, 0.01 \mathrm{mmol})$, pre-prepared tert-butylborate $2 \mathrm{a}(143.5 \mathrm{mg}$, $0.3 \mathrm{mmol})$, arylbromide $\mathbf{8 a}(46.6 \mathrm{mg}, 0.2 \mathrm{mmol})$ and DMA $(500 \mu \mathrm{l})$. After sealing the vial with parafilm, the reaction was placed in EvoluChem photoreactor (PhotoRedOx Duo) equiped with a 45W blue LED (Figure S1). After stirred for $14 \mathrm{~h}$, the reaction was quenched with water and extracted with diethyl ether (ca. $2 \mathrm{~mL} \times 3$ ). The combined organic extract was dried over $\mathrm{Na}_{2} \mathrm{SO}_{4}$ and after filtration, the filtrate was concentrated under reduced pressure. The residue was washed with $\mathrm{MeOH}$ to remove most of boracene and after $\mathrm{MeOH}$ were removed under reduced pressure, purified by flash column chromatography on silica gel gave 9 aa $(32.0 \mathrm{mg}, 76 \%)$ as a white solid.

(1)

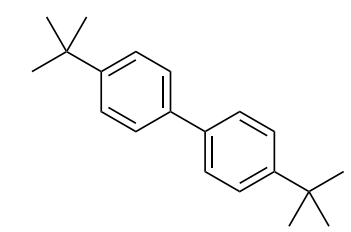

di-tert-butylbiphenyl (DBB) (2 equiv)
(2)

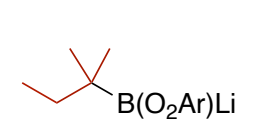

$2 e$
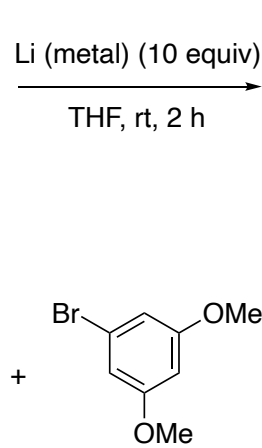

8d
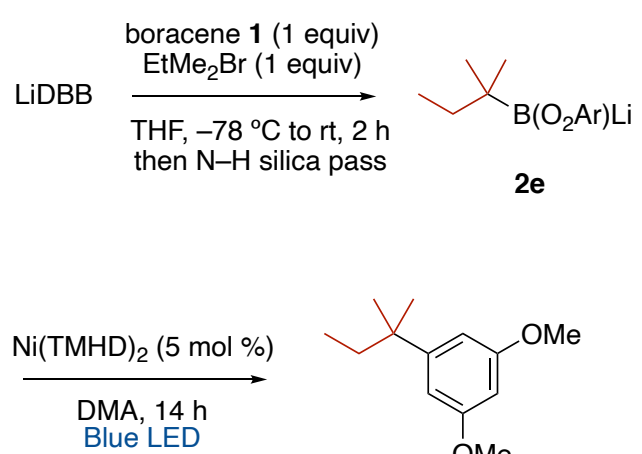

Blue LED

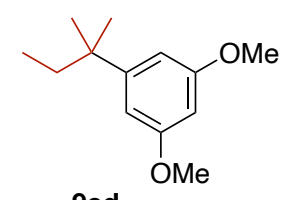

9ed

Method B (synthesis of 9ed as a representative): To a suspension of boracene 1 (54 $\mathrm{mg}, 0.2$ $\mathrm{mmol})$ and 2-bromo-2-methylbutane $(\mu \mathrm{l}, 0.2 \mathrm{mmol})$ in THF $(1.0 \mathrm{ml})$ was added LiDBB solution in THF (0.4 M), prepared from 4,4'-di-tert-butylbiphenyl (DBB, $107 \mathrm{mg}, 0.4 \mathrm{mmol}, 4$ equiv) and lithium wire (13.9 mg, $2.0 \mathrm{mmol}, 20$ equiv) by according to the literature ${ }^{5}$, at $-78{ }^{\circ} \mathrm{C}$ and then allowed to warm up to room temperature. After stirred for $2 \mathrm{~h}$, passed through a short plug of $\mathrm{N}-\mathrm{H}$ silica gel and THF was removed by spraying nitrogen gas to give tert-pentylborate $2 \mathbf{e}$. The borate was used without further purification.

In a glovebox, to an oven-dried vial with a stirring bar was added $\mathrm{Ni}(\mathrm{TMHD})_{2}(2.1 \mathrm{mg}, 5.0 \mu \mathrm{mol})$ and 1-bromo-3,5-dimethoxybenzene $8 \mathbf{d}(21.7 \mathrm{mg}, 0.100 \mathrm{mmol})$. To the mixture was added tertpentylborate 2e dissolved in DMA $(1.0 \mathrm{ml})$. After sealing the vial with parafilm, the reaction was placed in EvoluChem photoreactor (PhotoRedOx Duo) equiped with a 45W blue LED (Figure S1). After stirred for $24 \mathrm{~h}$, the reaction was quenched with water and extracted with diethyl ether (ca. 2 $\mathrm{mL} \times 3$ ). The combined organic extract was dried over $\mathrm{Na}_{2} \mathrm{SO}_{4}$ and after filtration, the filtrate was concentrated under reduced pressure. The residue was washed with $\mathrm{MeOH}$ to remove most of 
boracene and after $\mathrm{MeOH}$ were removed under reduced pressure, purified by flash column chromatography on silica gel gave 9ed (15.3 mg, 73\%) as a colorless oil.

(1)

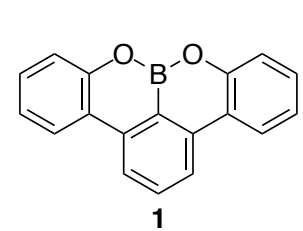

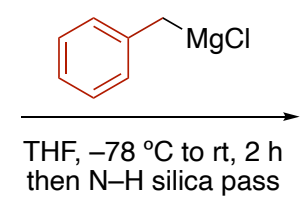

then $\mathrm{N}-\mathrm{H}$ silica pass

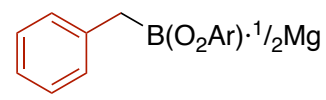

$2 \mathbf{b}$

(2)

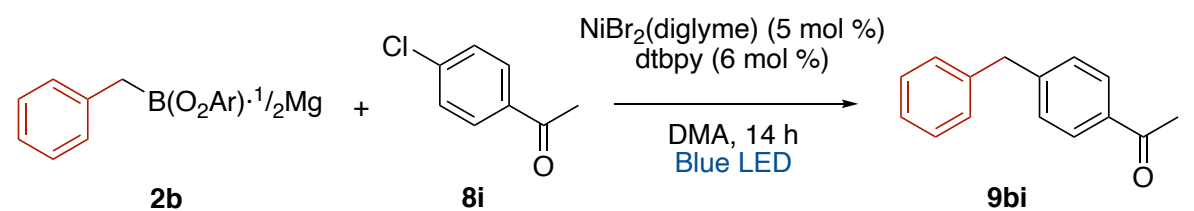

Method C (synthesis of 9bi as a representative): To a suspension of boracene 1 in THF (1.0 ml) was added benzylmagnesium chloride solution in THF $(1.0 \mathrm{M})$ prepared by conventional method at $-78^{\circ} \mathrm{C}$ and then allowed to warm up to room temperature. After stirred for $2 \mathrm{~h}$, passed through a short plug of $\mathrm{N}-\mathrm{H}$ silica gel to give benzylborate $\mathbf{2 b}$ in THF solution. The mixture was used without further purification.

In a glovebox, to an oven-dried vial with a stirring bar was added $\mathrm{NiBr}_{2} \cdot$ diglyme $(1.5 \mathrm{mg}, 5.0$ $\mu \mathrm{mol})$, dtbpy (4,4'-di-tert-butyl-2,2'-bipyridyl) (1.6 mg, $6.0 \mu \mathrm{mol})$ and 4'-chloroacetophenone $8 \mathbf{i}$ (15.5 $\mathrm{mg}, 0.1 \mathrm{mmol})$. To the mixture was added benzylborate $\mathbf{2 b}$ in THF solution $(1.0 \mathrm{~mL})$. After sealing the vial with parafilm, the reaction was placed in EvoluChem photoreactor (PhotoRedOx Duo) equiped with a $45 \mathrm{~W}$ blue LED (Figure S1). After stirred for $24 \mathrm{~h}$, the reaction was quenched with water and extracted with diethyl ether (ca. $2 \mathrm{~mL} \times 3$ ). The combined organic extract was dried over $\mathrm{Na}_{2} \mathrm{SO}_{4}$ and after filtration, the filtrate was concentrated under reduced pressure. The residue was washed with $\mathrm{MeOH}$ to remove most of boracene and after $\mathrm{MeOH}$ were removed under reduced pressure, purified by flash column chromatography on silica gel gave 9bi (18.8 $\mathrm{mg}, 89 \%)$ as a colorless oil.

\section{- Procedure for Ni-Catalyzed Three-Cmponent Coupling}

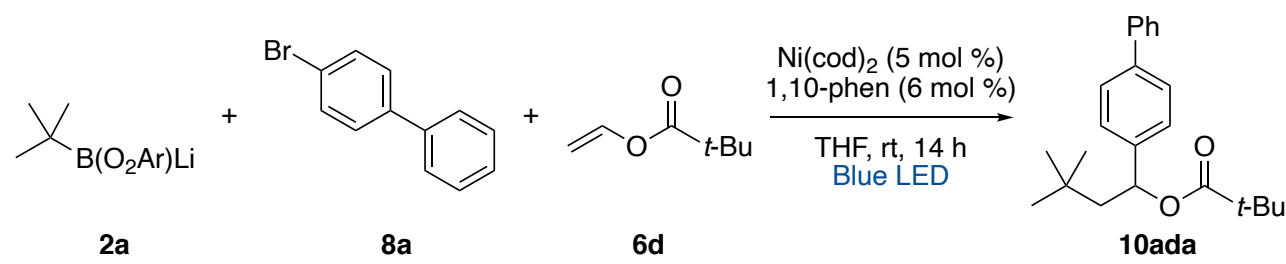

In a glovebox, to an oven-dried vial with a stirring bar was added $\mathrm{Ni}(\operatorname{cod})_{2}(1.4 \mathrm{mg}, 5.0 \mu \mathrm{mol})$ and 1,10-phenanthroline $(1.1 \mathrm{mg}, 6.0 \mu \mathrm{mol})$. To the mixture was added tert-butylborate $(\mathbf{2 a})(71.8 \mathrm{mg}$, $0.15 \mathrm{mmol})$, vinyl pivalate (6d) $(25.6 \mathrm{mg}, 29.1 \mu \mathrm{l}, 0.2 \mathrm{mmol}), 4$-bromo-1,1'-biphenyl (8a) (23.3 mg, $0.1 \mathrm{mmol})$ and THF $(1.0 \mathrm{ml})$. After sealing the vial with parafilm, the reaction was placed in EvoluChem photoreactor (PhotoRedOx Duo) equiped with a 45W blue LED (Figure S1). After stirred for $14 \mathrm{~h}$, the reaction was quenched with a short plug of silica gel using ethyl acetate. After volatiles were removed under reduced pressure, purification by flash column chromatography on silica gel gave the 10ada $(24.5 \mathrm{mg}, 72 \%)$ with the recovery of boracene $\mathbf{1}$ as a colorless solid. 


\section{1-(tert-butoxy)-2,2,6,6-tetramethylpiperidine (3a)}

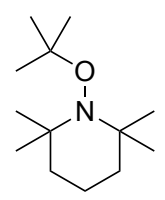

The product 3a was purified by flash chromatography on silica gel (100:0-90:10, hexane $/ \mathrm{CH}_{2} \mathrm{Cl}_{2}$ ) (Figure 1C; $27.5 \mathrm{mg}, 0.129 \mathrm{mmol}, 64 \%$ isolated yield).

${ }^{1} \mathbf{H}$ NMR $\left(400 \mathrm{MHz}, \mathrm{CDCl}_{3}\right) \delta 1.07(\mathrm{~s}, 6 \mathrm{H}), 1.12(\mathrm{~s}, 6 \mathrm{H}), 1.26(\mathrm{~s}, 9 \mathrm{H}), 1.23-1.28(\mathrm{~m}, 1 \mathrm{H}), 1.30$ $1.61(\mathrm{~m}, 5 \mathrm{H})$.

${ }^{13} \mathrm{C}$ NMR (100.5 MHz, $\left.\mathrm{CDCl}_{3}\right) \delta$ 17.2, 20.4 (2C), 29.4 (3C), 34.8 (2C), 40.8 (2C), 59.0 (2C), 77.1. The ${ }^{1} \mathrm{H}$ and ${ }^{13} \mathrm{C}$ NMR spectra data of $\mathbf{3 a}$ were consistent with the literature. ${ }^{6}$

\section{dimethyl 2-(tert-butyl)succinate (7aa)}

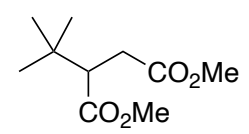

The product 7aa was purified by flash chromatography on silica gel (100:0-10:1, hexane/EtOAc) (Figure 1F; $14.0 \mathrm{mg}, 0.069 \mathrm{mmol}, 69 \%$ isolated yield).

${ }^{1} \mathbf{H}$ NMR $\left(400 \mathrm{MHz}, \mathrm{CDCl}_{3}\right) \delta 0.96(\mathrm{~s}, 9 \mathrm{H}), 2.48(\mathrm{dd}, J=2.0,11.2 \mathrm{~Hz}, 1 \mathrm{H}), 2.65(\mathrm{dd}, J=2.0,8.0$ $\mathrm{Hz}, 1 \mathrm{H}), 2.80(\mathrm{dd}, J=8.0,11.2 \mathrm{~Hz}, 1 \mathrm{H}), 3.66(\mathrm{~s}, 3 \mathrm{H}), 3.70(\mathrm{~s}, 3 \mathrm{H})$.

${ }^{13} \mathrm{C}$ NMR $\left(100.5 \mathrm{MHz}, \mathrm{CDCl}_{3}\right) \delta 27.8$ (4C), 32.6, 51.2, 51.4, 51.8, 173.2, 174.7.

The ${ }^{1} \mathrm{H}$ and ${ }^{13} \mathrm{C}$ NMR spectra data of 7 aa were consistent with the literature. ${ }^{7}$

\section{dimethyl 2-(sec-butyl)succinate (7ca)}

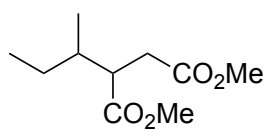

The product 7ca (1:1 mixture of diastereomers) was purified by flash chromatography on silica gel (100:0-10:1, hexane/EtOAc) (Figure 1F; $11.3 \mathrm{mg}, 0.056 \mathrm{mmol}, 56 \%$ isolated yield). Colorless oil.

IR (neat) 754, 845, 1005, 1165, 1253, 1347, 1437, 1734, $2961 \mathrm{~cm}^{-1}$.

${ }^{1} \mathbf{H}$ NMR $\left(600 \mathrm{MHz}, \mathrm{CDCl}_{3}\right) \delta 0.85-0.93(\mathrm{~m}, 3 \mathrm{H}), 1.14-1.46(\mathrm{~m}, 5 \mathrm{H}), 1.70(\mathrm{~m}, 0.5 \mathrm{H}), 1.83(\mathrm{~m}$, $0.5 \mathrm{H}), 2.35(\mathrm{dd}, J=3.6,16.8 \mathrm{~Hz}, 0.5 \mathrm{H}), 2.40(\mathrm{dd}, J=3.6,16.8 \mathrm{~Hz}, 0.5 \mathrm{H}), 2.75(\mathrm{~m}, 1 \mathrm{H}), 2.88(\mathrm{~m}$, $1 \mathrm{H}), 3.67(\mathrm{~d}, J=1.2 \mathrm{~Hz}, 3 \mathrm{H}), 3.70(\mathrm{~d}, J=3.0 \mathrm{~Hz}, 3 \mathrm{H})$.

${ }^{13} \mathbf{C}$ NMR $\left(150.9 \mathrm{MHz}, \mathrm{CDCl}_{3}\right) \delta 11.6,11.7,15.8,16.4,26.7,27.2,31.4,33.4,36.3,36.9,45.5$, 45.8, 51.6, 51.7, 51.8 (2C), 173.0, 173.1, 174.7, 175.2 .

HRMS-DART $(\mathrm{m} / \mathrm{z}):[\mathrm{M}+\mathrm{H}]^{+}$calcd for $\mathrm{C}_{10} \mathrm{H}_{19} \mathrm{O}_{4}{ }^{+}, 203.1283$; found, 203.1286 .

\section{dimethyl 2-butylsuccinate (7da)}

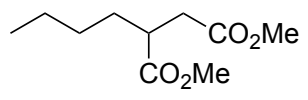


The product 7da was purified by flash chromatography on silica gel (100:0-10:1, hexane/EtOAc) (Figure 1F; $6.3 \mathrm{mg}, 0.031 \mathrm{mmol}, 31 \%$ isolated yield).

${ }^{1} \mathbf{H}$ NMR $\left(600 \mathrm{MHz}, \mathrm{CDCl}_{3}\right) \delta 0.88(\mathrm{t}, J=7.2 \mathrm{~Hz}, 3 \mathrm{H}), 1.14-1.38(\mathrm{~m}, 4 \mathrm{H}), 1.51(\mathrm{~m}, 1 \mathrm{H}), 1.64(\mathrm{~m}$, 1H), $2.44(\mathrm{dd}, J=4.8,16.8 \mathrm{~Hz}, 1 \mathrm{H}), 2.72(\mathrm{dd}, J=9.0,16.8 \mathrm{~Hz}, 1 \mathrm{H}), 2.84(\mathrm{~m}, 1 \mathrm{H}), 3.67$ (s, 3H), 3.70 $(\mathrm{s}, 3 \mathrm{H})$.

${ }^{13}$ C NMR (150.9 MHz, $\left.\mathrm{CDCl}_{3}\right) \delta 13.8,21.0,29.1,31.6,35.8,41.1,51.7,51.8,172.5,175.5$.

The ${ }^{1} \mathrm{H}$ and ${ }^{13} \mathrm{C}$ NMR spectra data of 7da were consistent with the literature. ${ }^{8}$

\section{4-(tert-butyl)-1,1'-biphenyl (9aa)}

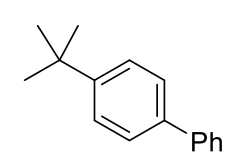

The product 9aa was synthesized according to Method A, and purified by flash chromatography on NH-silica gel (100:0-90:10, hexane/ $\mathrm{CH}_{2} \mathrm{Cl}_{2}$ ) (Table 1; $32.0 \mathrm{mg}, 0.15 \mathrm{mmol}, 76 \%$ isolated yield).

${ }^{1} \mathbf{H}$ NMR $\left(400 \mathrm{MHz}, \mathrm{CDCl}_{3}\right) \delta 1.37$ (s, 9H), $7.32(\mathrm{~m}, 1 \mathrm{H}), 7.41-7.48(\mathrm{~m}, 4 \mathrm{H}), 7.53-7.55(\mathrm{~m}, 2 \mathrm{H})$, 7.58-7.60 (m, 2H).

${ }^{13}$ C NMR (100.5 MHz, $\left.\mathrm{CDCl}_{3}\right) \delta 31.4$ (3C), 34.5, 125.7 (2C), $126.8(2 \mathrm{C}), 127.0,127.0$ (2C), 128.7 (2C), 138.3, 141.0, 150.2.

The ${ }^{1} \mathrm{H}$ and ${ }^{13} \mathrm{C}$ NMR spectra data of 9aa were consistent with the literature. ${ }^{9}$

\section{1-(tert-butyl)-4-methoxybenzene (9ab)}

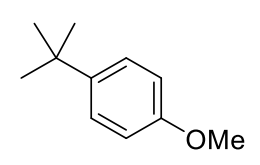

The product 9ab was synthesized according to Method A, and purified by flash chromatography on $\mathrm{NH}$-silica gel (100:0-90:10, hexane $/ \mathrm{CH}_{2} \mathrm{Cl}_{2}$ ) (Table 1; $23.9 \mathrm{mg}, 0.15 \mathrm{mmol}, 73 \%$ isolated yield, including isomerization product).

${ }^{1}$ H NMR $\left(400 \mathrm{MHz}, \mathrm{CDCl}_{3}\right) \delta 1.30(\mathrm{~s}, 9 \mathrm{H}), 3.80(\mathrm{~s}, 3 \mathrm{H}), 6.85(\mathrm{~d}, J=9.2 \mathrm{~Hz}, 2 \mathrm{H}), 7.31(\mathrm{~d}, J=9.2$ $\mathrm{Hz}, 2 \mathrm{H})$.

${ }^{13}$ C NMR (100.5 MHz, $\mathrm{CDCl}_{3}$ ) $\delta 31.5$ (3C), 34.0, 55.2, 113.3 (2C), 126.2 (2C), 143.3, 157.3.

The ${ }^{1} \mathrm{H}$ and ${ }^{13} \mathrm{C}$ NMR spectra data of product 9ab were consistent with the literature. ${ }^{9}$

\section{4-(tert-butyl)-N,N-diethylaniline (9ac)}

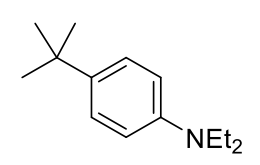

The product 9ac was synthesized according to Method A, and purified by flash chromatography on $\mathrm{NH}$-silica gel (100:0-90:10, hexane $/ \mathrm{CH}_{2} \mathrm{Cl}_{2}$ ) (Table 1; $25.2 \mathrm{mg}, 0.12 \mathrm{mmol}, 61 \%$ isolated yield, including isomerization product). Pale orange oil.

IR (neat) 812, 1205, 1263, 1361, 1373, 1518, 1614, $2962 \mathrm{~cm}^{-1}$. 
${ }^{1} \mathbf{H}$ NMR $\left(600 \mathrm{MHz}, \mathrm{CDCl}_{3}\right) \delta 1.15(\mathrm{t}, J=7.2 \mathrm{~Hz}, 6 \mathrm{H}), 1.28(\mathrm{~s}, 9 \mathrm{H}), 3.32(\mathrm{q}, J=7.2 \mathrm{~Hz}, 4 \mathrm{H}), 6.64$ $(\mathrm{d}, J=9.0 \mathrm{~Hz}, 2 \mathrm{H}), 7.24(\mathrm{~d}, J=9.0 \mathrm{~Hz}, 2 \mathrm{H})$.

${ }^{13} \mathrm{C}$ NMR (150.9 MHz, $\left.\mathrm{CDCl}_{3}\right) \delta 12.7$ (2C), 31.6 (3C), 33.6, 44.3 (2C), 111.5 (2C), 126.0 (2C), 137.9, 145.6.

HRMS-DART $(\mathrm{m} / \mathrm{z}):[\mathrm{M}+\mathrm{H}]^{+}$calcd for $\mathrm{C}_{14} \mathrm{H}_{24} \mathrm{~N}^{+}$, 206.1903; found, 206.1907.

\section{1-(tert-butyl)-3,5-dimethoxybenzene (9ad)}<smiles>COc1cc(OC)cc(C(C)(C)C)c1</smiles>

The product 9ad was synthesized according to Method A, and purified by flash chromatography on NH-silica gel (100:0-90:10, hexane $/ \mathrm{CH}_{2} \mathrm{Cl}_{2}$ ) (Table 1; $31.0 \mathrm{mg}, 0.16 \mathrm{mmol}, 80 \%$ isolated yield).

${ }^{1} \mathrm{H}$ NMR $\left(400 \mathrm{MHz}, \mathrm{CDCl}_{3}\right) \delta 1.30(\mathrm{~s}, 9 \mathrm{H}), 3.80(\mathrm{~s}, 6 \mathrm{H}), 6.31(\mathrm{t}, J=2.0 \mathrm{~Hz}, 1 \mathrm{H}), 6.55(\mathrm{~d}, J=2.0$ $\mathrm{Hz}, 2 \mathrm{H})$.

${ }^{13}$ C NMR (100.5 MHz, $\mathrm{CDCl}_{3}$ ) $\delta 31.2$ (3C), 34.9, 55.2 (2C), 96.7, 104.0 (2C), 153.8, 160.5 (2C).

The ${ }^{1} \mathrm{H}$ and ${ }^{13} \mathrm{C}$ NMR spectra data of product 9ad were consistent with the literature. ${ }^{9}$

\section{1,3,5-tri-tert-butylbenzene (9ae)}

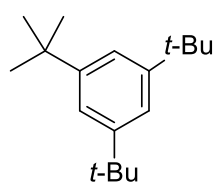

The product 9ae was synthesized according to Method A, and purified by flash chromatography on $\mathrm{NH}$-silica gel (100:0-90:10, hexane/ $\mathrm{CH}_{2} \mathrm{Cl}_{2}$ ) (Table 1; $34.3 \mathrm{mg}, 0.14 \mathrm{mmol}, 70 \%$ isolated yield, including isomerization product).

${ }^{1} \mathbf{H}$ NMR $\left(400 \mathrm{MHz}, \mathrm{CDCl}_{3}\right) \delta 1.34(\mathrm{~s}, 27 \mathrm{H}), 7.26(\mathrm{~s}, 3 \mathrm{H})$.

${ }^{13}$ C NMR (100.5 MHz, CDCl $) \delta 31.6$ (9C), 35.0 (3C), 119.5 (3C), 149.9 (3C).

The ${ }^{1} \mathrm{H}$ and ${ }^{13} \mathrm{C}$ NMR spectra data of product 9ae were consistent with the literature. ${ }^{10}$

\section{1-(tert-butyl)-4-(trifluoromethoxy)benzene (9af)}

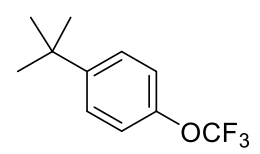

The product 9af synthesized according to Method A (Table $1 ; 69 \%{ }^{1} \mathrm{H}$ NMR yield).

${ }^{1} \mathbf{H}$ NMR $\left(600 \mathrm{MHz}, \mathrm{CDCl}_{3}\right) \delta 1.32(\mathrm{~s}, 9 \mathrm{H}), 7.13(\mathrm{~d}, J=8.4 \mathrm{~Hz}, 2 \mathrm{H}), 7.39$ (d, $\left.J=8.4 \mathrm{~Hz}, 2 \mathrm{H}\right)$.

${ }^{13} \mathrm{C} \mathrm{NMR}\left(150.9 \mathrm{MHz}, \mathrm{CDCl}_{3}\right) \delta 31.3,(3 \mathrm{C}), 34.5,120.4(2 \mathrm{C}), 120.4$ (q, $\left.J=270.0 \mathrm{~Hz}\right), 126.6(2 \mathrm{C})$, 146.9, 149.8 .

${ }^{19}$ F NMR $\left(376 \mathrm{MHz}, \mathrm{CDCl}_{3}\right) \delta-56.6$.

The ${ }^{1} \mathrm{H},{ }^{13} \mathrm{C}$, and, ${ }^{19} \mathrm{~F}$ NMR spectra data of product 9af were consistent with the literature. ${ }^{11}$

\section{4-(tert-butyl)benzonitrile (9ag)}




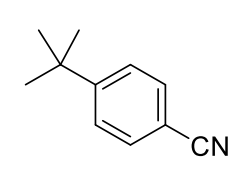

The product 9ag was syntheesized according to $\operatorname{Method~} \mathbf{A}$, and purified by flash chromatography on $\mathrm{NH}$-silica gel (100:0-90:10, hexane/ $\mathrm{CH}_{2} \mathrm{Cl}_{2}$ ) (Table 1; $13.3 \mathrm{mg}, 0.084 \mathrm{mmol}, 42 \%$ isolated yield).

${ }^{1} \mathbf{H}$ NMR $\left(600 \mathrm{MHz}, \mathrm{CDCl}_{3}\right) \delta 1.33(\mathrm{~s}, 9 \mathrm{H}), 7.48(\mathrm{~d}, J=8.4 \mathrm{~Hz}, 2 \mathrm{H}), 7.59(\mathrm{~d}, J=8.4 \mathrm{~Hz}, 2 \mathrm{H})$.

${ }^{13}$ C NMR (100.5 MHz, $\left.\mathrm{CDCl}_{3}\right) \delta 30.9$ (3C), 35.3, 109.3, 119.2, 126.2 (2C), 132.0 (2C), 156.6.

The ${ }^{1} \mathrm{H}$ and ${ }^{13} \mathrm{C}$ NMR spectra data of product 9ag were consistent with the literature. ${ }^{12}$

\section{4-(tert-butyl)benzaldehyde (9ah)}

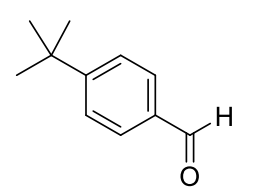

The product 9ah was synthesized according to Method A, and purified by flash chromatography on silica gel (100:0-80:20, hexane/ $\mathrm{CH}_{2} \mathrm{Cl}_{2}$ ) (Table 1; $13.0 \mathrm{mg}, 0.080 \mathrm{mmol}, 40 \%$ isolated yield, including isomerization product and inseparable impurity).

${ }^{1} \mathbf{H}$ NMR $\left(400 \mathrm{MHz}, \mathrm{CDCl}_{3}\right) \delta 1.36(\mathrm{~s}, 9 \mathrm{H}), 7.56(\mathrm{~d}, J=8.4 \mathrm{~Hz}, 2 \mathrm{H}), 7.82(\mathrm{~d}, J=8.4 \mathrm{~Hz}, 2 \mathrm{H})$, $9.99(\mathrm{~s}, 1 \mathrm{H})$.

${ }^{13}$ C NMR (100.5 MHz, $\left.\mathrm{CDCl}_{3}\right) \delta 31.1$ (3C), 35.3, 126.0 (2C), 129.7 (2C), 134.1, 158.4, 192.1.

The ${ }^{1} \mathrm{H}$ and ${ }^{13} \mathrm{C}$ NMR spectra data of product 9ah were consistent with the literature. ${ }^{12}$

\section{1-(4-(tert-butyl)phenyl)ethan-1-one (9ai)}

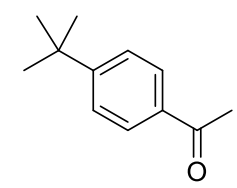

The product 9ai was synthesized according to Method A, and purified by flash chromatography on NH-silica gel (100:0-90:10, hexane $/ \mathrm{CH}_{2} \mathrm{Cl}_{2}$ ) (Table 1; $25.4 \mathrm{mg}, 0.14 \mathrm{mmol}, 72 \%$ isolated yield, including isomerization product).

${ }^{1}$ H NMR $\left(400 \mathrm{MHz}, \mathrm{CDCl}_{3}\right) \delta 1.35(\mathrm{~s}, 9 \mathrm{H}), 2.59(\mathrm{~s}, 3 \mathrm{H}), 7.48(\mathrm{~d}, J=8.4 \mathrm{~Hz}, 2 \mathrm{H}), 7.90(\mathrm{~d}, J=8.4$ $\mathrm{Hz}, 2 \mathrm{H})$.

${ }^{13}$ C NMR (100.5 MHz, $\left.\mathrm{CDCl}_{3}\right) \delta 26.5,31.1$ (3C), 35.1, 125.5 (2C), 128.3 (2C), 134.6, 156.8, 197.9.

The ${ }^{1} \mathrm{H}$ and ${ }^{13} \mathrm{C}$ NMR spectra data of product 9ai were consistent with the literature. ${ }^{12}$

\section{1-(tert-butyl)-4-(methylsulfonyl)benzene (9aj)}

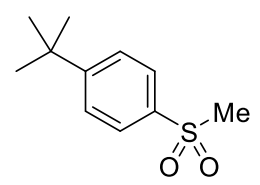

The product 9aj was synthesized according to Method A, and purified by flash chromatography on NH-silica gel (100:0-90:10, hexane $/ \mathrm{CH}_{2} \mathrm{Cl}_{2}$ ) (Table 1; $24.1 \mathrm{mg}, 0.11 \mathrm{mmol}, 57 \%$ isolated yield).

${ }^{1} \mathbf{H}$ NMR $\left(400 \mathrm{MHz}, \mathrm{CDCl}_{3}\right) \delta 1.36(\mathrm{~s}, 9 \mathrm{H}), 3.05(\mathrm{~s}, 3 \mathrm{H}), 7.58(\mathrm{~d}, J=8.4 \mathrm{~Hz}, 2 \mathrm{H}), 7.87$ (d, $J=8.4$ $\mathrm{Hz}, 2 \mathrm{H})$. 
${ }^{13}$ C NMR (100.5 MHz, $\mathrm{CDCl}_{3}$ ) $\delta 31.0$ (3C), 35.2, 44.6, 126.3 (2C), 127.2 (2C), 137.6, 157.6.

The ${ }^{1} \mathrm{H}$ and ${ }^{13} \mathrm{C}$ NMR spectra data of product 9aj were consistent with the literature. ${ }^{11}$

methyl 4-(tert-butyl)benzoate (9ak)

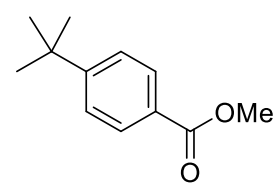

The product 9ak was synthesized according to Method A, and purified by flash chromatography on $\mathrm{NH}$-silica gel (100:0-90:10, hexane $/ \mathrm{CH}_{2} \mathrm{Cl}_{2}$ ) (Table 1; $23.5 \mathrm{mg}, 0.12 \mathrm{mmol}, 61 \%$ isolated yield).

${ }^{1} \mathbf{H}$ NMR $\left(400 \mathrm{MHz} \mathrm{CDCl}_{3}\right) \delta 1.34(\mathrm{~s}, 9 \mathrm{H}), 3.90(\mathrm{~s}, 3 \mathrm{H}), 7.45(\mathrm{~d}, J=8.8 \mathrm{~Hz}, 2 \mathrm{H}), 7.97(\mathrm{~d}, J=8.8$ $\mathrm{Hz}, 2 \mathrm{H})$.

${ }^{13}$ C NMR (100.5 MHz, $\left.\mathrm{CDCl}_{3}\right) \delta 31.1$ (3C), 35.0, 51.9, 125.3 (2C), 127.3, 129.4 (2C), 156.5, 167.1.

The ${ }^{1} \mathrm{H}$ and ${ }^{13} \mathrm{C}$ NMR spectra data of product 9ak were consistent with the literature. ${ }^{13}$

\section{2-(4-(tert-butyl)phenyl)-4,4,5,5-tetramethyl-1,3,2-dioxaborolane (9al)}

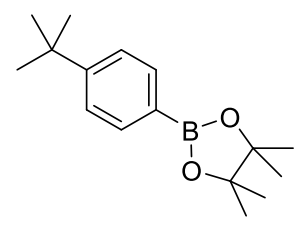

The product 9al was synthesized according to $\mathbf{M e t h o d ~ A , ~ a n d ~ p u r i f i e d ~ b y ~ f l a s h ~ c h r o m a t o g r a p h y ~}$ on silica gel (100:0-85:15, hexane/toluene) (Table 1; $27.0 \mathrm{mg}, 0.10 \mathrm{mmol}, 52 \%$ isolated yield, including isomerization product).

${ }^{1} \mathbf{H}$ NMR $\left(400 \mathrm{MHz}, \mathrm{CDCl}_{3}\right) \delta 1.32(\mathrm{~s}, 9 \mathrm{H}), 1.33(\mathrm{~s}, 12 \mathrm{H}), 7.41(\mathrm{~d}, J=8.0 \mathrm{~Hz}, 2 \mathrm{H}), 7.76(\mathrm{~d}, J=$ $8.0 \mathrm{~Hz}, 2 \mathrm{H})$.

${ }^{13}$ C NMR (100.5 MHz, $\left.\mathrm{CDCl}_{3}\right) \delta 24.8$ (4C), 31.2 (3C), 34.9, 83.6 (2C), 124.7 (2C), 134.7 (2C), 154.5. The signal for the carbon attached to the boron atom was not observed.

The ${ }^{1} \mathrm{H}$ and ${ }^{13} \mathrm{C}$ NMR spectra data of product 9al were consistent with the literature. ${ }^{14}$

\section{(E)-(3,3-dimethylbut-1-en-1-yl)benzene (9am)}

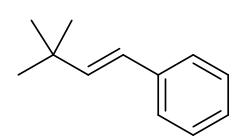

The product 9am synthesized according to Method A (Table 1; $65 \%{ }^{1} \mathrm{H}$ NMR yield).

${ }^{1} \mathbf{H}$ NMR $\left(400 \mathrm{MHz}, \mathrm{CDCl}_{3}\right) \delta 1.12(\mathrm{~s}, 9 \mathrm{H}), 6.25(\mathrm{~d}, J=16.4 \mathrm{~Hz}, 1 \mathrm{H}), 6.31(\mathrm{~d}, J=16.4 \mathrm{~Hz}, 1 \mathrm{H})$, $7.19(\mathrm{~m}, 1 \mathrm{H}), 7.24-7.31(\mathrm{~m}, 2 \mathrm{H}), 7.36(\mathrm{~d}, J=7.6 \mathrm{~Hz}, 2 \mathrm{H})$.

${ }^{13} \mathrm{C}$ NMR (100.5 MHz, $\left.\mathrm{CDCl}_{3}\right) \delta 29.6$ (3C), 33.3, 124.5, 126.0 (2C), 126.7, 128.5 (2C), 138.0, 141.8 .

The ${ }^{1} \mathrm{H}$ and ${ }^{13} \mathrm{C}$ NMR spectra data of product 9am were consistent with the literature. ${ }^{15}$

\section{2-(tert-butyl)naphthalene (9an)}




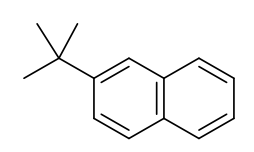

The product 9an was synthesized according to Method A, and purified by flash chromatography on $\mathrm{NH}-$ silica gel (100:0-90:10, hexane $\left./ \mathrm{CH}_{2} \mathrm{Cl}_{2}\right)$ (Table 1; $21.1 \mathrm{mg}, 0.11 \mathrm{mmol}, 57 \%$ isolated yield).

${ }^{1} \mathbf{H}$ NMR $\left(400 \mathrm{MHz}, \mathrm{CDCl}_{3}\right) \delta 1.42(\mathrm{~s}, 9 \mathrm{H}), 7.41-7.45(\mathrm{~m}, 2 \mathrm{H}), 7.58(\mathrm{~m}, 1 \mathrm{H}), 7.76-7.82(\mathrm{~m}, 4 \mathrm{H})$.

${ }^{13} \mathbf{C}$ NMR (100.5 MHz, $\left.\mathrm{CDCl}_{3}\right) \delta 31.2$ (3C), 34.8, 122.8, 124.8, 125.2, 125.8, 127.3, 127.5, 127.9, 131.6, 133.3, 148.5.

The ${ }^{1} \mathrm{H}$ and ${ }^{13} \mathrm{C}$ NMR spectra data of product 9an were consistent with the literature. ${ }^{9}$

\section{1,3-dimethoxy-5-(tert-pentyl)benzene (9ed)}

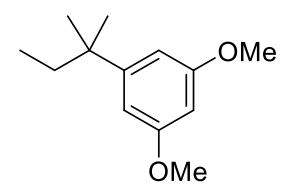

The product 9ed was synthesized according to Method B using 2-bromo-2-methylbutane with LiDBB, and purified by flash chromatography on silica gel (100:0-80:20, hexane/ $\mathrm{CH}_{2} \mathrm{Cl}_{2}$ ) (Table 1; $15.2 \mathrm{mg}, 0.073 \mathrm{mmol}, 73 \%$ isolated yield). Colorless oil.

IR (neat) 831, 1155, 1205, 1422, 1456, 1595, 2835, $2962 \mathrm{~cm}^{-1}$.

${ }^{1} \mathbf{H}$ NMR (400 MHz, $\left.\mathrm{CDCl}_{3}\right) \delta 0.69$ (t, $\left.J=7.2 \mathrm{~Hz}, 3 \mathrm{H}\right), 1.25(\mathrm{~s}, 6 \mathrm{H}), 1.61$ (q, $\left.J=7.2 \mathrm{~Hz}, 2 \mathrm{H}\right), 3.80$ (s, 6H), 6.30 (t, $J=2.4 \mathrm{~Hz}, 1 \mathrm{H}), 6.49$ (d, $J=2.4 \mathrm{~Hz}, 2 \mathrm{H})$.

${ }^{13} \mathrm{C}$ NMR (150.9 MHz, $\left.\mathrm{CDCl}_{3}\right) \delta$ 9.1, 28.4 (2C), 36.8, 38.2, 55.2 (2C), 96.6, 104.8 (2C), 152.2, $160.4(2 \mathrm{C})$.

HRMS-DART $(\mathrm{m} / \mathrm{z}):[\mathrm{M}+\mathrm{H}]^{+}$calcd for $\mathrm{C}_{13} \mathrm{H}_{21} \mathrm{O}_{2}{ }^{+}, 209.1536$; found, 209.1545 .

\section{1,3-dimethoxy-5-(2-methyl-1-phenylpropan-2-yl)benzene (9fd)}

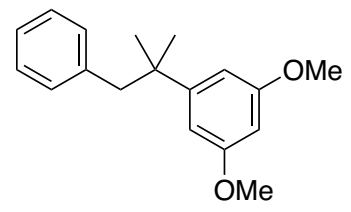

The product 9fd was synthesized according to Method B using 2-bromo-2-methyl-1phenylpropane with LiDBB, and purified by flash chromatography on silica gel (100:0-80:20, hexane $/ \mathrm{CH}_{2} \mathrm{Cl}_{2}$ ) (Table 1; $14.4 \mathrm{mg}, 0.053 \mathrm{mmol}, 53 \%$ isolated yield). Colorless oil.

IR (neat) 702, 831, 1056, 1155, 1204, 1341, 1422, 1455, 1495, $2961 \mathrm{~cm}^{-1}$.

${ }^{1} \mathrm{H}$ NMR $\left(600 \mathrm{MHz}, \mathrm{CDCl}_{3}\right) \delta 1.28(\mathrm{~s}, 6 \mathrm{H}), 2.84(\mathrm{~s}, 2 \mathrm{H}), 3.77(\mathrm{~s}, 6 \mathrm{H}), 6.33(\mathrm{~s}, 1 \mathrm{H}), 6.45(\mathrm{~s}, 2 \mathrm{H})$, 6.87-6.89 (m, 2H), 7.14-7.17 (m, 3H).

${ }^{13} \mathrm{C}$ NMR (150.9 MHz, $\left.\mathrm{CDCl}_{3}\right) \delta 28.2(2 \mathrm{C}), 39.0,50.8,55.2(2 \mathrm{C}), 97.1,105.0(2 \mathrm{C}), 125.9,127.5$ (2C), 130.4 (2C), 138.8, 151.8, $160.3(2 \mathrm{C})$.

HRMS-DART $(\mathrm{m} / \mathrm{z})$ : [M] ${ }^{+}$calcd for $\mathrm{C}_{18} \mathrm{H}_{22} \mathrm{O}_{2}{ }^{+}, 270.1614$; found, 270.1616 .

\section{1,3-dimethoxy-5-(2-(methyl- $\left.d_{3}\right)$ propan-2-yl-1,1,1,3,3,3- $\left.d_{6}\right)$ benzene (9gd)}




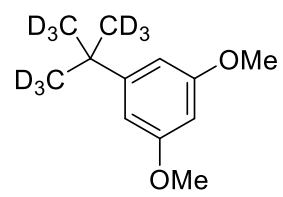

The product 9gd was synthesized according to Method B using 2-chloro-2-methylpropane- $d_{9}$ $(99.3 \% \mathrm{D})$ with LiDBB, and purified by flash chromatography on silica gel (100:0-80:10, hexane $/ \mathrm{CH}_{2} \mathrm{Cl}_{2}$ ) (Table 1; $8.3 \mathrm{mg}, 0.041 \mathrm{mmol}, 41 \%$ isolated yield, 98.9\%D). Colorless oil.

IR (neat) 852, 1072, 1154, 1204, 1456, 1595, 2215, $2937 \mathrm{~cm}^{-1}$.

${ }^{1} \mathbf{H}$ NMR $\left(400 \mathrm{MHz}, \mathrm{CDCl}_{3}\right) \delta 3.80(\mathrm{~s}, 6 \mathrm{H}), 6.31(\mathrm{t}, J=2.4 \mathrm{~Hz}, 1 \mathrm{H}), 6.54(\mathrm{t}, J=2.4 \mathrm{~Hz}, 2 \mathrm{H})$.

${ }^{13}$ C NMR (100.5 MHz, $\left.\mathrm{CDCl}_{3}\right) \delta 55.2$ (2C), 96.7, 104.1 (2C), 160.5 (2C).

HRMS-DART $(\mathrm{m} / z)$ : $[\mathrm{M}+\mathrm{H}]^{+}$calcd for $\mathrm{C}_{12} \mathrm{H}_{10} \mathrm{D}_{9} \mathrm{O}_{2}{ }^{+}, 204.1944$; found, 204.1951.

\section{(3r,5r,7r)-1-(3,5-dimethoxyphenyl)adamantane (9hd)}

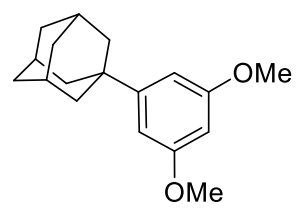

The product 9hd was synthesized according to Method B using 1-bromoadamantane with LiDBB, and purified by flash chromatography on silica gel (100:0-80:10, hexane/ $\left.\mathrm{CH}_{2} \mathrm{Cl}_{2}\right)$ (Table 1; $17.5 \mathrm{mg}$, $0.064 \mathrm{mmol}, 64 \%$ isolated yield). Pale yellow oil.

IR (neat) 694, 1150, 1203, 1453, 1593, 2846, $2899 \mathrm{~cm}^{-1}$.

${ }^{1}$ H NMR $\left(400 \mathrm{MHz}, \mathrm{CDCl}_{3}\right) \delta 1.72-1.80(\mathrm{~m}, 6 \mathrm{H}), 1.90(\mathrm{~s}, 6 \mathrm{H}), 2.08(\mathrm{~s}, 3 \mathrm{H}), 3.80(\mathrm{~s}, 6 \mathrm{H}), 6.31(\mathrm{t}$, $J=2.4 \mathrm{~Hz}, 1 \mathrm{H}), 6.53(\mathrm{~d}, J=2.4 \mathrm{~Hz}, 2 \mathrm{H})$.

${ }^{13}$ C NMR (150.9 MHz, $\left.\mathrm{CDCl}_{3}\right) \delta 28.9(3 \mathrm{C}), 36.5,36.8(3 \mathrm{C}), 43.1$ (3C), $55.2(2 \mathrm{C}), 97.0,103.5(2 \mathrm{C})$, $154.1,160.5(2 \mathrm{C})$.

HRMS-DART $(\mathrm{m} / \mathrm{z}):[\mathrm{M}+\mathrm{H}]^{+}$calcd for $\mathrm{C}_{18} \mathrm{H}_{25} \mathrm{O}_{2}{ }^{+}, 273.1849$; found, 273.1850.

\section{4-(sec-butyl)-1,1'-biphenyl (9ca)}

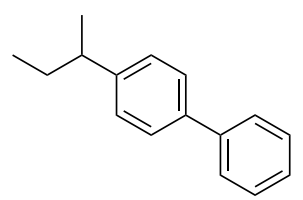

The product 9ca was synthesized according to Method A using sec-butyllithium, and purified by flash chromatography on silica gel (100:0-90:10, hexane $/ \mathrm{CH}_{2} \mathrm{Cl}_{2}$ ) (Table 1; $12.4 \mathrm{mg}, 0.059 \mathrm{mmol}$, $59 \%$ isolated yield).

${ }^{1} \mathbf{H}$ NMR $\left(400 \mathrm{MHz}, \mathrm{CDCl}_{3}\right) \delta 0.86(\mathrm{t}, J=7.8 \mathrm{~Hz}, 3 \mathrm{H}), 1.27(\mathrm{~d}, J=7.3 \mathrm{~Hz}, 3 \mathrm{H}), 1.63(\mathrm{dq}, J=6.9$, $7.8 \mathrm{~Hz}, 2 \mathrm{H}), 2.64$ (tq, $J=6.9,7.3 \mathrm{~Hz}, 1 \mathrm{H}), 7.26(\mathrm{~d}, J=8.2 \mathrm{~Hz}, 2 \mathrm{H}), 7.32$ (dd, $J=7.3,7.3 \mathrm{~Hz}, 1 \mathrm{H})$, $7.43(\mathrm{dd}, J=7.3,7.8 \mathrm{~Hz}, 2 \mathrm{H}), 7.52(\mathrm{~d}, J=8.3 \mathrm{~Hz}, 2 \mathrm{H}), 7.60(\mathrm{~d}, J=8.2 \mathrm{~Hz}, 2 \mathrm{H})$.

${ }^{13} \mathrm{C}$ NMR (100.5 MHz, $\left.\mathrm{CDCl}_{3}\right) \delta 12.3,21.8,31.2,41.3,126.9,127.0$ (4C), 127.4 (2C), 128.7 (2C), $138.7,141.2,146.8$.

The ${ }^{1} \mathrm{H}$ and ${ }^{13} \mathrm{C}$ NMR spectra data of product 9ca were consistent with the literature. ${ }^{16}$ 


\section{4-isopropyl-1,1'-biphenyl (9ia)}

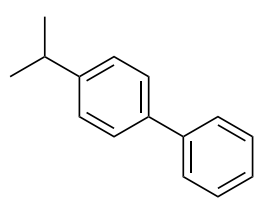

The product 9ia was synthesized according to Method $\mathbf{C}$ using iso-propylmagnesium bromide, and purified by flash chromatography on silica gel (100:0-90:10, hexane/ $\left.\mathrm{CH}_{2} \mathrm{Cl}_{2}\right)$ (Table 1; $12.3 \mathrm{mg}$, $0.063 \mathrm{mmol}, 63 \%$ isolated yield).

${ }^{1} \mathbf{H}$ NMR $\left(400 \mathrm{MHz} \mathrm{CDCl}_{3}\right) \delta 1.29(\mathrm{~d}, J=6.9 \mathrm{~Hz}, 6 \mathrm{H}), 2.96(\mathrm{sep}, J=6.9 \mathrm{~Hz}, 1 \mathrm{H}), 7.28-7.35$ (m, $3 \mathrm{H}), 7.43(\mathrm{dd}, J=7.3,7.8 \mathrm{~Hz}, 2 \mathrm{H}), 7.53(\mathrm{~d}, J=8.2 \mathrm{~Hz}, 2 \mathrm{H}), 7.58(\mathrm{~d}, J=8.2 \mathrm{~Hz}, 2 \mathrm{H})$.

${ }^{13} \mathrm{C}$ NMR (100.5 MHz, $\left.\mathrm{CDCl}_{3}\right) \delta 24.0(2 \mathrm{C}), 33.8,126.8$ (2C), 126.9 (2C), $127.0(2 \mathrm{C}), 127.1,128.7$ (2C), 138.7, 141.2, 148.0 .

The ${ }^{1} \mathrm{H}$ and ${ }^{13} \mathrm{C}$ NMR spectra data of product 9ia were consistent with the literature. ${ }^{16}$

\section{4-cyclohexyl-1,1'-biphenyl (9ja)}

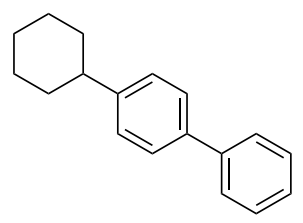

The product 9ja was synthesized according to Method $\mathbf{C}$ using cyclohexylmagnesium bromide, and purified by flash chromatography on silica gel (100:0-90:10, hexane $/ \mathrm{CH}_{2} \mathrm{Cl}_{2}$ ) (Table 1; $8.3 \mathrm{mg}$, $0.035 \mathrm{mmol}, 35 \%$ isolated yield).

${ }^{1} \mathbf{H}$ NMR $\left(400 \mathrm{MHz}, \mathrm{CDCl}_{3}\right) \delta 1.22-1.48(\mathrm{~m}, 5 \mathrm{H}), 1.77(\mathrm{~m}, 1 \mathrm{H}), 1.82-195(\mathrm{~m}, 4 \mathrm{H}), 2.55(\mathrm{~m}, 1 \mathrm{H})$, $7.28(\mathrm{~d}, J=8.2 \mathrm{~Hz}, 2 \mathrm{H}), 7.32(\mathrm{dd}, J=7.3,7.3 \mathrm{~Hz}, 1 \mathrm{H}), 7.42(\mathrm{dd}, J=7.3,7.8 \mathrm{~Hz}, 2 \mathrm{H}), 7.52(\mathrm{~d}, J=$ $7.3 \mathrm{~Hz}, 2 \mathrm{H}), 7.58(\mathrm{~d}, J=7.8 \mathrm{~Hz}, 2 \mathrm{H})$.

${ }^{13} \mathrm{C}$ NMR (100.5 MHz, $\left.\mathrm{CDCl}_{3}\right) \delta 26.2,26.9$ (2C), 34.4 (2C), 44,2, 126.9, 127.0 (4C), 127.2 (2C), 128.7 (2C), 138.7, 141.2, 147.2 .

The ${ }^{1} \mathrm{H}$ and ${ }^{13} \mathrm{C}$ NMR spectra data of product 9 ja were consistent with the literature. ${ }^{17}$

\section{4-cyclopentyl-1,1'-biphenyl (9ka)}

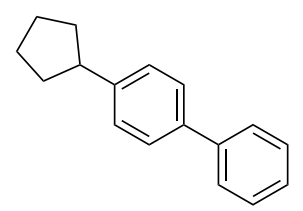

The product 9ka was synthesized according to Method $\mathbf{C}$ using cyclopentylmagnesium chloride, and purified by flash chromatography on silica gel (100:0-90:10, hexane/ $\left.\mathrm{CH}_{2} \mathrm{Cl}_{2}\right)$ (Table 1; $10.9 \mathrm{mg}$, $0.049 \mathrm{mmol}, 49 \%$ isolated yield).

${ }^{1} \mathbf{H}$ NMR $\left(400 \mathrm{MHz}, \mathrm{CDCl}_{3}\right) \delta 1.58-1.75(\mathrm{~m}, 4 \mathrm{H}), 1.78-1.85(\mathrm{~m}, 2 \mathrm{H}), 2.05-2.30(\mathrm{~m}, 2 \mathrm{H}), 3.03$ (quint., $J=7.8 \mathrm{~Hz}, 1 \mathrm{H}), 7.30-7.34(\mathrm{~m}, 3 \mathrm{H}), 7.39$ (dd, $J=7.8,7.8 \mathrm{~Hz}, 2 \mathrm{H}), 7.54(\mathrm{~d}, J=8.2 \mathrm{~Hz}, 2 \mathrm{H})$, $7.58(\mathrm{~d}, J=7.3 \mathrm{~Hz}, 2 \mathrm{H})$. 
${ }^{13}$ C NMR (100.5 MHz, $\left.\mathrm{CDCl}_{3}\right) \delta 25.5$ (2C), $34.6(2 \mathrm{C}), 45.6,126.97,126.99$ (4C), 127.5 (2C), 128.7 (2C), 138.6, 141.2, 145.7 .

The ${ }^{1} \mathrm{H}$ and ${ }^{13} \mathrm{C}$ NMR spectra data of product 9 ka were consistent with the literature. ${ }^{17}$

\section{4-cyclobutyl-1,1'-biphenyl (9ia)}

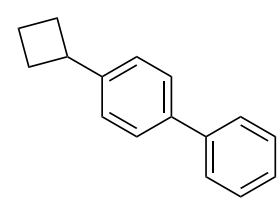

The product 9ia was synthesized according to Method $\mathbf{C}$ using cyclobutylmagnesium bromide, and purified by flash chromatography on silica gel (100:0-90:10, hexane/ $\mathrm{CH}_{2} \mathrm{Cl}_{2}$ ) (Table 1; $14.3 \mathrm{mg}$, $0.069 \mathrm{mmol}, 69 \%$ isolated yield).

${ }^{1}$ H NMR $\left(400 \mathrm{MHz}, \mathrm{CDCl}_{3}\right) \delta 1.88(\mathrm{~m}, 1 \mathrm{H}), 2.04(\mathrm{~m}, 1 \mathrm{H}), 2.15-2.25(\mathrm{~m}, 2 \mathrm{H}), 2.34-2.44(\mathrm{~m}, 2 \mathrm{H})$, 3.59 (quint., $J=8.7 \mathrm{~Hz}, 1 \mathrm{H}), 7.30-7.34(\mathrm{~m}, 3 \mathrm{H}), 7.39$ (dd, $J=7.8,7.8 \mathrm{~Hz}, 2 \mathrm{H}), 7.54$ (d, $J=8.2 \mathrm{~Hz}$, $2 \mathrm{H}), 7.58(\mathrm{~d}, J=7.3 \mathrm{~Hz}, 2 \mathrm{H})$.

${ }^{13}$ C NMR (100.5 MHz, $\left.\mathrm{CDCl}_{3}\right) \delta 18.3,29.8$ (2C), 40.1, 126.7, 126.95 (2C), 126.99 (2C), 127.0 (2C), 128.7 (2C), 138.7, 141.2, 145.4.

The ${ }^{1} \mathrm{H}$ and ${ }^{13} \mathrm{C}$ NMR spectra data of product 9ia were consistent with the literature. ${ }^{17}$

\section{1-(4-butylphenyl)ethan-1-one (9di)}

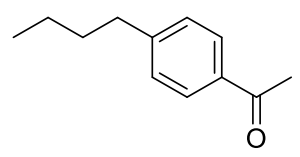

The product 9di was synthesized according to Method A using $n$-butyllithium, and purified by flash chromatography on $\mathrm{NH}-$ silica gel (100:0-90:10, hexane $/ \mathrm{CH}_{2} \mathrm{Cl}_{2}$ ) (Table 1; $18.0 \mathrm{mg}, 0.10 \mathrm{mmol}$, $51 \%$ isolated yield).

${ }^{1} \mathbf{H}$ NMR $\left(400 \mathrm{MHz}, \mathrm{CDCl}_{3}\right) \delta 0.93(\mathrm{t}, J=7.2 \mathrm{~Hz}, 3 \mathrm{H}), 1.31-1.40(\mathrm{~m}, 2 \mathrm{H}), 1.58-1.65(\mathrm{~m}, 2 \mathrm{H})$, $2.58(\mathrm{~s}, 3 \mathrm{H}), 2.66(\mathrm{t}, J=7.2 \mathrm{~Hz}, 2 \mathrm{H}), 7.26(\mathrm{~d}, J=8.4 \mathrm{~Hz}, 2 \mathrm{H}), 7.88(\mathrm{~d}, J=8.4 \mathrm{~Hz}, 2 \mathrm{H})$.

${ }^{13}$ C NMR (150.9 MHz, $\left.\mathrm{CDCl}_{3}\right) \delta 13.9,22.3,26.6,33.2,35.7,128.5$ (2C), $128.6(2 \mathrm{C}), 134.9,148.8$, 197.9.

The ${ }^{1} \mathrm{H}$ and ${ }^{13} \mathrm{C}$ NMR spectra data of product 9di were consistent with the literature. ${ }^{18}$

\section{1-(4-phenethylphenyl)ethan-1-one (9mi)}

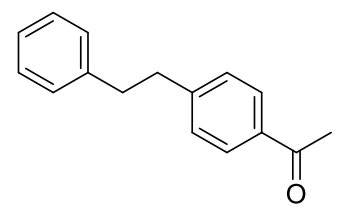

The product $9 \mathrm{mi}$ was synthesized according to Method $\mathbf{C}$ using phenylethylmagnesium bromide, and purified by flash chromatography on silica gel (100:0-90:10, hexane $/ \mathrm{CH}_{2} \mathrm{Cl}_{2}$ ) (Table 1; $9.4 \mathrm{mg}$, $0.042 \mathrm{mmol}, 42 \%$ isolated yield). 
${ }^{1} \mathbf{H}$ NMR $\left(400 \mathrm{MHz}, \mathrm{CDCl}_{3}\right) \delta 2.59(\mathrm{~s}, 3 \mathrm{H}), 2.91-.01(\mathrm{~m}, 4 \mathrm{H}), 7.15-7.30(\mathrm{~m}, 7 \mathrm{H}), 7.87(\mathrm{~d}, J=8.4$ $\mathrm{Hz}, 2 \mathrm{H})$.

${ }^{13}$ C NMR $\left(100.5 \mathrm{MHz}, \mathrm{CDCl}_{3}\right) \delta 26.6,37.4,37.8,126.1,128.39$ (2C), $128.42(2 \mathrm{C}), 128.5$ (2C), 128.7 (2C), 135.1, 141.1, 147.5, 197.9.

The ${ }^{1} \mathrm{H}$ and ${ }^{13} \mathrm{C}$ NMR spectra data of product $9 \mathrm{mi}$ were consistent with the literature. ${ }^{19}$

\section{1-(4-(but-3-en-1-yl)phenyl)ethan-1-one (9ni)}

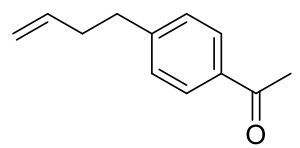

The product 9ni was synthesized according to Method C using 3-buten-1-ylmagnesium bromide, and purified by flash chromatography on silica gel (100:0-90:10, hexane/ $\mathrm{CH}_{2} \mathrm{Cl}_{2}$ ) (Table 1; $5.4 \mathrm{mg}$, $0.031 \mathrm{mmol}, 31 \%$ isolated yield, including inseparable isomerization product). Colorless oil.

IR (neat) 818, 956, 1268, 1358, 1607, 1683, 2358, $2926 \mathrm{~cm}^{-1}$.

${ }^{1} \mathbf{H}$ NMR (600 MHz, $\left.\mathrm{CDCl}_{3}\right) \delta 2.39$ (q, $\left.J=7.2 \mathrm{~Hz}, 2 \mathrm{H}\right), 2.59(\mathrm{~s}, 3 \mathrm{H}), 2.77(\mathrm{t}, J=7.2 \mathrm{~Hz}, 2 \mathrm{H})$, $4.98-5.05$ (m, 2H), $5.83(\mathrm{~m}, 1 \mathrm{H}), 7.28$ (d, $J=8.4 \mathrm{~Hz}, 2 \mathrm{H}), 7.88(\mathrm{~d}, J=8.4 \mathrm{~Hz}, 2 \mathrm{H})$.

${ }^{13}$ C NMR (150.9 MHz, $\left.\mathrm{CDCl}_{3}\right) \delta$ 26.5, 35.0, 35.3, 115.4, 128.5 (2C), 128.6 (2C), 135.0, 137.5, 147.6, 197.9.

HRMS-DART $(\mathrm{m} / \mathrm{z}):[\mathrm{M}+\mathrm{H}]^{+}$calcd for $\mathrm{C}_{12} \mathrm{H}_{15} \mathrm{O}^{+}, 175.1117$; found, 175.1121 .

\section{1-(4-benzylphenyl)ethan-1-one (9bi)}

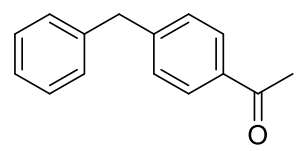

The product $9 \mathrm{bi}$ was synthesized according to Method $\mathbf{C}$, and purified by flash chromatography on silica gel (100:0-90:10, hexane/ $\mathrm{CH}_{2} \mathrm{Cl}_{2}$ ) (Table 1; $18.8 \mathrm{mg}, 0.089 \mathrm{mmol}, 89 \%$ isolated yield).

${ }^{1} \mathbf{H}$ NMR $\left(400 \mathrm{MHz}, \mathrm{CDCl}_{3}\right) \delta 2.58(\mathrm{~s}, 3 \mathrm{H}), 4.04(\mathrm{~s}, 2 \mathrm{H}), 7.18-7.30(\mathrm{~m}, 7 \mathrm{H}), 7.89(\mathrm{~d}, J=8.0 \mathrm{~Hz}$, $2 \mathrm{H})$.

${ }^{13} \mathrm{C}$ NMR (100.5 MHz, $\left.\mathrm{CDCl}_{3}\right) \delta 26.6,41.9,126.4$ (2C), 128.6 (2C), 128.9 (2C), 129.0, 129.1 (2C), 135.2, 140.0, 146.8, 197.9.

The ${ }^{1} \mathrm{H}$ and ${ }^{13} \mathrm{C}$ NMR spectra data of product 9 bi were consistent with the literature. ${ }^{19}$

\section{1-(p-tolyl)ethan-1-one (9oi)}

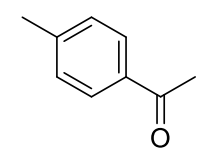

The product 9oi was synthesized according to Method $\mathbf{C}$ using methyllithium, and purified by flash chromatography on silica gel (100:0-90:10, hexane/ $\mathrm{CH}_{2} \mathrm{Cl}_{2}$ ) (Table 1; $9.8 \mathrm{mg}, 0.073 \mathrm{mmol}$, $73 \%$ isolated yield).

${ }^{1} \mathbf{H}$ NMR $\left(600 \mathrm{MHz}, \mathrm{CDCl}_{3}\right) \delta 2.41(\mathrm{~s}, 3 \mathrm{H}), 2.58(\mathrm{~s}, 3 \mathrm{H}), 7.26(\mathrm{~d}, J=8.0 \mathrm{~Hz}, 2 \mathrm{H}), 7.86(\mathrm{~d}, J=8.0$ $\mathrm{Hz}, 2 \mathrm{H})$. 
${ }^{13}$ C NMR $\left(100.5 \mathrm{MHz}, \mathrm{CDCl}_{3}\right) \delta 22.1,26.9,128.8$ (2C), 129.6, 129.7, 135.1, 144.3, 198.3.

The ${ }^{1} \mathrm{H}$ and ${ }^{13} \mathrm{C}$ NMR spectra data of product 9oi were consistent with the literature. ${ }^{20}$

\section{3-methylquinoline (90o)}

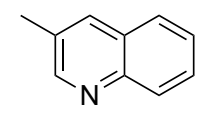

The product 900 was synthesized according to Method $\mathbf{C}$ using methyllithium, and purified by flash chromatography on silica gel (100:0-90:10, hexane $/ \mathrm{CH}_{2} \mathrm{Cl}_{2}$ ) (Table 1; $16.0 \mathrm{mg}, 0.112 \mathrm{mmol}$, $56 \%$ isolated yield).

${ }^{1} \mathbf{H}$ NMR $\left(400 \mathrm{MHz}, \mathrm{CDCl}_{3}\right) \delta 2.53(\mathrm{~s}, 3 \mathrm{H}), 7.51(\mathrm{~m}, 1 \mathrm{H}), 7.65(\mathrm{~m}, 1 \mathrm{H}), 7.75(\mathrm{~d}, J=8.4 \mathrm{~Hz}, 1 \mathrm{H})$, $7.93(\mathrm{~s}, 1 \mathrm{H}), 8.07(\mathrm{~d}, J=8.4 \mathrm{~Hz}, 1 \mathrm{H}), 8.78(\mathrm{~d}, J=2.0 \mathrm{~Hz}, 1 \mathrm{H})$.

${ }^{13} \mathrm{C}$ NMR $\left(100.5 \mathrm{MHz}, \mathrm{CDCl}_{3}\right) \delta 18.8,126.5,127.1,128.1,128.4,129.2,130.5,134.7,146.6$, 152.4 .

The ${ }^{1} \mathrm{H}$ and ${ }^{13} \mathrm{C}$ NMR spectra data of product 900 were consistent with the literature. ${ }^{21}$

\section{7-methyl-2H-chromen-2-one (9op)}

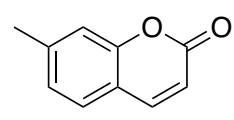

The product 9op was synthesized according to Method $\mathbf{C}$ using methyllithium, and purified by flash chromatography on silica gel (100:0-95:5, $\left.\mathrm{CH}_{2} \mathrm{Cl}_{2} / \mathrm{MeOH}\right)$ (Table 1; $24.3 \mathrm{mg}, 0.152 \mathrm{mmol}, 76 \%$ isolated yield, including impurity).

The ${ }^{1} \mathrm{H}$ spectra data of product 9op was consistent with the literature. ${ }^{22}$

methyl 2-(5-methoxy-2-methyl-1-(4-methylbenzoyl)-1 H-indol-3-yl)acetate (9op)

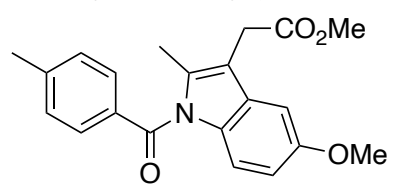

The product 9op was synthesized according to Method $\mathbf{C}$ using methyllithium, and purified by flash chromatography on silica gel (100:0-90:10, hexane/EtOAc) (Table 1; $28.2 \mathrm{mg}, 0.08 \mathrm{mmol}, 80 \%$ isolated yield).

${ }^{1} \mathbf{H}$ NMR $\left(600 \mathrm{MHz}, \mathrm{CDCl}_{3}\right) \delta 2.40(\mathrm{~s}, 3 \mathrm{H}), 2.47(\mathrm{~s}, 3 \mathrm{H}), 3.68(\mathrm{~s}, 2 \mathrm{H}), 3.71(\mathrm{~s}, 3 \mathrm{H}), 3.85(\mathrm{~s}, 3 \mathrm{H})$, $6.66(\mathrm{dd}, J=2.4,8.9 \mathrm{~Hz}, 1 \mathrm{H}), 6.89(\mathrm{~d}, J=8.9 \mathrm{~Hz}, 1 \mathrm{H}), 6.97(\mathrm{~d}, J=2.4 \mathrm{~Hz}, 1 \mathrm{H}), 7.29(\mathrm{~d}, J=8.2 \mathrm{~Hz}$, $2 \mathrm{H}), 7.63(\mathrm{~d}, J=8.2 \mathrm{~Hz}, 2 \mathrm{H})$.

${ }^{13} \mathrm{C}$ NMR $\left(150.9 \mathrm{MHz}, \mathrm{CDCl}_{3}\right) \delta 13.2,21.7,30.2,52.1,55.7,101.0,111.4,111.8,115.0,129.4$ (2C), 130.0 (2C), 130.4, 131.0, 132.7, 136.1, 143.7, 155.8, 169.4, 171.5.

The ${ }^{1} \mathrm{H}$ and ${ }^{13} \mathrm{C}$ NMR spectra data of product 9op were consistent with the literature. ${ }^{21}$

tert-butyl 2-([1,1'-biphenyl]-4-yl)-4,4-dimethylpentanoate (9aba) 


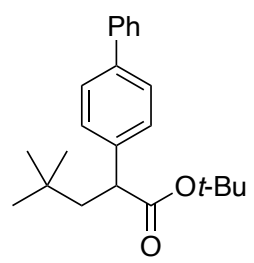

The product 9aba was purified by flash chromatography on silica gel (100:0-10:1, hexane $\left./ \mathrm{CH}_{2} \mathrm{Cl}_{2}\right)$ (Table 2; $11.7 \mathrm{mg}, 0.035 \mathrm{mmol}, 35 \%$ isolated yield). White solid.

M.p. : $84-86^{\circ} \mathrm{C}$

IR (neat) 669, 1142, 1731, 2341, 2957, $3734 \mathrm{~cm}^{-1}$.

${ }^{1}$ H NMR $\left(400 \mathrm{MHz}, \mathrm{CDCl}_{3}\right) \delta 0.93(\mathrm{~s}, 9 \mathrm{H}), 1.39$ (s, 9H), $1.53(\mathrm{dd}, J=3.6,14.4 \mathrm{~Hz}, 1 \mathrm{H}), 2.30(\mathrm{dd}$, $J=9.2,14.4 \mathrm{~Hz}, 1 \mathrm{H}), 3.58(\mathrm{dd}, J=3.6,9.2 \mathrm{~Hz}, 1 \mathrm{H}), 7.33(\mathrm{t}, J=7.2 \mathrm{~Hz}, 1 \mathrm{H}), 7.37(\mathrm{~d}, J=8.4 \mathrm{~Hz}$, 2H), 7.43 (dd, $J=7.2,7.8 \mathrm{~Hz}, 2 \mathrm{H}) 7.53(\mathrm{~d}, J=8.4 \mathrm{~Hz}, 2 \mathrm{H}), 7.58(\mathrm{~d}, J=7.8 \mathrm{~Hz}, 2 \mathrm{H})$.

${ }^{13}$ C NMR (100.5 MHz, $\left.\mathrm{CDCl}_{3}\right) \delta 27.9$ (3C), 29.5 (3C), 31.1, 47.3, 48.9, 80.4, 127.0 (4C), 128.0 (4C), 128.7 (2C), 139.5, 140.7, 173.9.

HRMS-DART $(\mathrm{m} / \mathrm{z}):[\mathrm{M}+\mathrm{H}]^{+}$calcd for $\mathrm{C}_{23} \mathrm{H}_{31} \mathrm{O}_{2}{ }^{+}, 339.2319$; found, 339.2317.

tert-butyl 2-[(1,1'-biphenyl)-4-yl]-4-methylhexanoate (10cba)

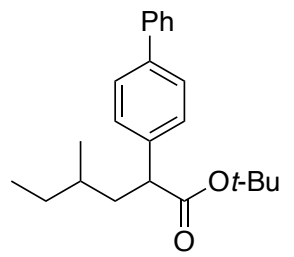

The product 10cba was purified by flash chromatography on silica gel (100:0-10:1, hexane $/ \mathrm{CH}_{2} \mathrm{Cl}_{2}$ ) (Table 2; $21.8 \mathrm{mg}, 0.64 \mathrm{mmol}, 64 \%$ isolated yield, 1:1 diastereomixture and including impurity). Colorless oil.

IR (neat) 506, 561, 648, 697, 732, 759, 843, 908, 1145, 1258, 1367, 1457, 1487, 1726, 2874, 2928, $2961 \mathrm{~cm}^{-1}$.

${ }^{1} \mathbf{H}$ NMR $\left(400 \mathrm{MHz}, \mathrm{CDCl}_{3}\right) \delta 0.82-0.95(\mathrm{~m}, 7 \mathrm{H}), 1.12-1.35(\mathrm{~m}, 1.5 \mathrm{H}), 1.37-1.51(\mathrm{~m}, 1 \mathrm{H}), 1.41$ $(\mathrm{s}, 4.5 \mathrm{H}), 1.42(\mathrm{~s}, 4.5 \mathrm{H}), 1.83(\mathrm{t}, J=6.7 \mathrm{~Hz}, 1 \mathrm{H}), 2.11-2.17(\mathrm{~m}, 0.5 \mathrm{H}), 3.57-3.64(\mathrm{~m}, 1 \mathrm{H}), 7.31-7.46$ $(\mathrm{m}, 5 \mathrm{H}), 7.55(\mathrm{~d}, J=8.2 \mathrm{~Hz}, 2 \mathrm{H}), 7.60(\mathrm{dd}, J=1.8,6.0 \mathrm{~Hz}, 2 \mathrm{H})$.

${ }^{13} \mathrm{C} \mathrm{NMR}\left(100.5 \mathrm{MHz}, \mathrm{CDCl}_{3}\right) \delta 11.13$ (0.5C), $11.14(0.5 \mathrm{C}), 18.9(0.5 \mathrm{C}), 19.1$ (0.5C), 27.97 (1.5C), 27.99 (1.5C), 29.4 (0.5C), 29.5 (0.5C), 32.0 (0.5C), 32.4 (0.5C), 40.2 (0.5C), 40.9 (0.5C), 50.09 (0.5C), $50.14(0.5 \mathrm{C}), 80.50(0.5 \mathrm{C}), 80.54(0.5 \mathrm{C}), 127.00(2 \mathrm{C}), 127.09(2 \mathrm{C}), 127.12(1 \mathrm{C}), 128.2(1 \mathrm{C})$, 128.3 (1C), 128.7 (1C), 138.9 (1C), 139.4 (1C), 139.64 (0.5C), 139.66 (0.5C), 140.8 (1C), 173.4 (0.5C), $173.7(0.5 \mathrm{C})$.

HRMS-DART $(\mathrm{m} / \mathrm{z})$ : $[\mathrm{M}]^{+}$calcd for $\mathrm{C}_{23} \mathrm{H}_{30} \mathrm{O}_{2}{ }^{+}, 338.2252$; found, 338.2240. 


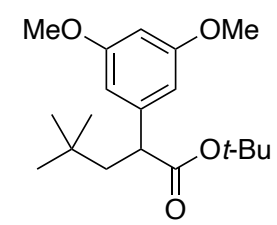

The product 10abd was purified by flash chromatography on silica gel (100:0-10:1, hexane $/ \mathrm{CH}_{2} \mathrm{Cl}_{2}$ ) (Table 2; $8.3 \mathrm{mg}, 0.026 \mathrm{mmol}, 26 \%$ isolated yield, including inseparable impurity). Colorless oil.

IR (neat) 669, 1142, 1206, 1596, 1729, 2341, $2928 \mathrm{~cm}^{-1}$.

${ }^{1}$ H NMR (400 MHz, $\left.\mathrm{CDCl}_{3}\right) \delta 0.91(\mathrm{~s}, 9 \mathrm{H}), 1.39(\mathrm{~s}, 9 \mathrm{H}), 1.46(\mathrm{dd}, J=2.8,15.6 \mathrm{~Hz}, 1 \mathrm{H}), 2.23(\mathrm{dd}$, $J=9.2,14.2 \mathrm{~Hz}, 1 \mathrm{H}), 3.45(\mathrm{dd}, J=3.2,9.6 \mathrm{~Hz}, 1 \mathrm{H}), 3.78(\mathrm{~s}, 6 \mathrm{H}), 6.33(\mathrm{t}, J=1.8 \mathrm{~Hz}, 1 \mathrm{H}), 6.46(\mathrm{~d}$, $J=2.4 \mathrm{~Hz}, 2 \mathrm{H})$.

${ }^{13}$ C NMR (100.5 MHz, $\left.\mathrm{CDCl}_{3}\right) \delta 27.9$ (3C), 29.5 (3C), 29.7, 31.0, 47.2, 55.3 (2C), 80.4, 98.7, 105.7 (2C), 139.4, 160.7 (2C), 173.5 .

HRMS-DART $(\mathrm{m} / \mathrm{z}):[\mathrm{M}+\mathrm{H}]^{+}$calcd for $\mathrm{C}_{19} \mathrm{H}_{31} \mathrm{O}_{4}{ }^{+}, 323.2222$; found, 323.2214 .

\section{tert-butyl 2-(4-acetylphenyl)-4,4-dimethylpentanoate (10abi)}

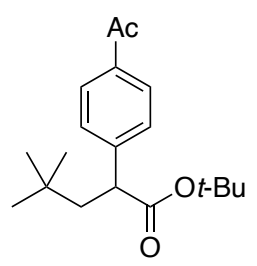

The product 10abi was purified by flash chromatography on silica gel (100:0-10:1, hexane $/ \mathrm{CH}_{2} \mathrm{Cl}_{2}$ ) (Table 2; $16.9 \mathrm{mg}, 0.056 \mathrm{mmol}, 56 \%$ isolated yield). Pale orange solid.

M.p. : $54-56{ }^{\circ} \mathrm{C}$

IR (neat) 669, 1144, 1685, 1732, 2341, 2957, $3733 \mathrm{~cm}^{-1}$.

${ }^{1} \mathbf{H}$ NMR $\left(400 \mathrm{MHz}, \mathrm{CDCl}_{3}\right) \delta 0.91(\mathrm{~s}, 9 \mathrm{H}), 1.37$ (s, 9H), $1.50(\mathrm{dd}, J=3.6,14.0 \mathrm{~Hz}, 1 \mathrm{H}), 2.28(\mathrm{dd}$, $J=8.8,14.0 \mathrm{~Hz}, 1 \mathrm{H}), 2.59$ (s, 3H), $3.60(\mathrm{dd}, J=3.6,8.8 \mathrm{~Hz}, 1 \mathrm{H}), 7.40(\mathrm{~d}, J=8.4 \mathrm{~Hz}, 2 \mathrm{H}), 7.90(\mathrm{~d}$, $J=8.4 \mathrm{~Hz}, 2 \mathrm{H})$.

${ }^{13}$ C NMR (100.5 MHz, $\left.\mathrm{CDCl}_{3}\right) \delta 26.6,27.8$ (3C), 29.5 (3C), 31.1, 47.0, 49.4, 80.8, 127.9 (2C), 128.6 (2C), 135.7, 147.1, 173.1, 197.8 .

HRMS-DART $(\mathrm{m} / z):[\mathrm{M}+\mathrm{H}]^{+}$calcd for $\mathrm{C}_{19} \mathrm{H}_{29} \mathrm{O}_{3}{ }^{+}, 305.2111$; found, 305.2106.

\section{2-(1-([1,1'-biphenyl]-4-yl)-3,3-dimethylbutyl)-4,4,5,5-tetramethyl-1,3,2-dioxaborolane (10aca)}

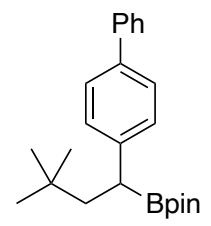

The product 10aca was purified by flash chromatography on silica gel (100:0-10:1, hexane $/ \mathrm{CH}_{2} \mathrm{Cl}_{2}$ ) (Table 2; $24.4 \mathrm{mg}, 0.067 \mathrm{mmol}, 67 \%$ isolated yield, including inseparable impurity). 
${ }^{1} \mathbf{H}$ NMR (400 MHz, $\left.\mathrm{CDCl}_{3}\right) \delta 0.92(\mathrm{~s}, 9 \mathrm{H}), 1.157(\mathrm{~s}, 6 \mathrm{H}), 1.160(\mathrm{~s}, 6 \mathrm{H}), 1.53(\mathrm{dd}, J=3.6,13.6$ $\mathrm{Hz}, 1 \mathrm{H}), 2.04(\mathrm{dd}, J=3.2,6.8 \mathrm{~Hz}, 1 \mathrm{H}), 2.44(\mathrm{dd}, J=3.6,10.0 \mathrm{~Hz}, 1 \mathrm{H}), 7.29-7.32(\mathrm{~m}, 3 \mathrm{H}), 7.41$ (t, $J=7.6 \mathrm{~Hz}, 2 \mathrm{H}), 7.49(\mathrm{~d}, J=8.4 \mathrm{~Hz}, 2 \mathrm{H}), 7.59(\mathrm{~d}, J=7.2 \mathrm{~Hz}, 2 \mathrm{H})$.

${ }^{13} \mathrm{C}$ NMR (100.5 MHz, $\left.\mathrm{CDCl}_{3}\right) \delta 24.4$ (2C), 24.6 (2C), 29.7 (3C), 30.2, 46.6, 83.3 (2C), 127.07, 127.12 (2C), 127.3 (2C), 128.6 (2C), 128.8 (2C), 137.7, 141.2, 144.1.

The ${ }^{1} \mathrm{H}$ and ${ }^{13} \mathrm{C}$ NMR spectra data of product 10aca were consistent with the literature. ${ }^{23}$

\section{2-(1-(3,5-dimethoxyphenyl)-3,3-dimethylbutyl)-4,4,5,5-tetramethyl-1,3,2-dioxaborolane} (10acd)

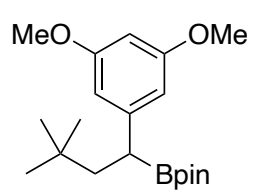

The product 10acd was purified by flash chromatography on silica gel (100:0-1:1, hexane/ $\left.\mathrm{CH}_{2} \mathrm{Cl}_{2}\right)$ (Table 2; $13.3 \mathrm{mg}, 0.038 \mathrm{mmol}, 38 \%$ isolated yield).

${ }^{1} \mathbf{H}$ NMR (400 MHz, $\left.\mathrm{CDCl}_{3}\right) \delta 0.90$ (s, 9H), 1.165 (s, 6H), $1.168(\mathrm{~s}, 6 \mathrm{H}), 1.47$ (dd, $J=3.6,13.2$ $\mathrm{Hz}, 1 \mathrm{H}), 1.98(\mathrm{dd}, J=10.0,13.2 \mathrm{~Hz}, 1 \mathrm{H}), 2.32(\mathrm{dd}, J=3.2,10.0 \mathrm{~Hz}, 1 \mathrm{H}), 3.77(\mathrm{~s}, 6 \mathrm{H}), 6.24(\mathrm{t}, J=$ $2.4 \mathrm{~Hz}, 1 \mathrm{H}), 6.41(\mathrm{~d}, J=2.4 \mathrm{~Hz}, 2 \mathrm{H})$.

${ }^{13} \mathrm{C}$ NMR (100.5 MHz, $\left.\mathrm{CDCl}_{3}\right) \delta 24.4$ (2C), 24.7 (2C), 29.6 (3C), 31.3, 46.6, $55.2(2 \mathrm{C}), 83.2(2 \mathrm{C})$, 97.4, 106.1 (2C), 147.3, 160.5 (2C).

The ${ }^{1} \mathrm{H}$ and ${ }^{13} \mathrm{C}$ NMR spectra data of product 10acd were consistent with the literature. ${ }^{23}$

\section{1-([1,1'-biphenyl]-4-yl)-3,3-dimethylbutyl pivalate (10ada)}

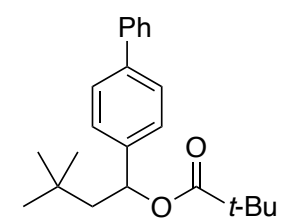

The product 10ada was purified by flash chromatography on silica gel (100:0-10:1, hexane $/ \mathrm{CH}_{2} \mathrm{Cl}_{2}$ ) (Table 2; $24.5 \mathrm{mg}, 0.072 \mathrm{mmol}, 72 \%$ isolated yield). White solid.

M.p. : $93-95{ }^{\circ} \mathrm{C}$

IR (neat) 405, 669, 1151, 1728, 1978, 2342, 2359, $2959 \mathrm{~cm}^{-1}$.

${ }^{1}$ H NMR (400 MHz, $\left.\mathrm{CDCl}_{3}\right) \delta 0.98(\mathrm{~s}, 9 \mathrm{H}), 1.20(\mathrm{~s}, 9 \mathrm{H}), 1.61(\mathrm{dd}, J=2.8,14.8 \mathrm{~Hz}, 1 \mathrm{H}), 2.01$ (dd, $J=9.2,14.8 \mathrm{~Hz}, 1 \mathrm{H}), 5.85(\mathrm{dd}, J=2.8,9.2 \mathrm{~Hz}, 1 \mathrm{H}), 7.31-7.37(\mathrm{~m}, 3 \mathrm{H}), 7.34(\mathrm{t}, J=7.6 \mathrm{~Hz}, 2 \mathrm{H})$, $7.55-7.58(\mathrm{~m}, 4 \mathrm{H})$.

${ }^{13}$ C NMR (100.5 MHz, $\left.\mathrm{CDCl}_{3}\right) \delta 27.1$ (3C), 30.0 (3C), 30.5, 38.6, 50.4, 73.7, 126.3, 127.1 (2C), 127.2 (4C), 128.7 (2C), 140.3, 140.8, 142.1, 177.6.

HRMS-DART $(m / z):\left[\mathrm{M}+\mathrm{NH}_{4}\right]^{+}$calcd for $\mathrm{C}_{23} \mathrm{H}_{34} \mathrm{~N}_{1} \mathrm{O}_{2}{ }^{+}, 356.2590$; found, 356.2593.

\section{1-(3,5-dimethoxyphenyl)-3,3-dimethylbutyl pivalate (10add)}

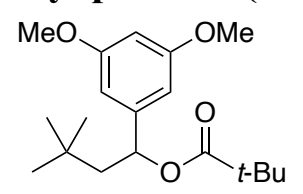


The product 10add was purified by flash chromatography on silica gel (100:0-7:3, hexane/ $\left.\mathrm{CH}_{2} \mathrm{Cl}_{2}\right)$ (Table 2; $16.7 \mathrm{mg}, 0.052 \mathrm{mmol}, 52 \%$ isolated yield). Pale yellow oil.

IR (neat) 669, 1155, 1205, 1598, 1729, 2341, $2957 \mathrm{~cm}^{-1}$.

${ }^{1} \mathbf{H}$ NMR $\left(400 \mathrm{MHz}, \mathrm{CDCl}_{3}\right) \delta 0.96$ (s, 9H), 1.20 (s, 9H), 1.55 (dd, $\left.J=2.4,14.8 \mathrm{~Hz}, 1 \mathrm{H}\right), 1.92(\mathrm{dd}$, $J=9.6,14.8 \mathrm{~Hz}, 1 \mathrm{H}), 3.77(\mathrm{~s}, 6 \mathrm{H}), 5.72(\mathrm{dd}, J=2.4,9.6 \mathrm{~Hz}, 1 \mathrm{H}), 6.33(\mathrm{t}, J=2.4 \mathrm{~Hz}, 1 \mathrm{H}), 6.43(\mathrm{~d}$, $J=2.4 \mathrm{~Hz}, 2 \mathrm{H})$.

${ }^{13}$ C NMR (100.5 MHz, $\left.\mathrm{CDCl}_{3}\right) \delta 27.1$ (3C), 29.9 (3C), 30.5, 38.6, 50.5, 55.3 (2C), 73.8, 99.2, 103.6 (2C), 145.7, $160.8(2 \mathrm{C}), 177.5$.

HRMS-DART $(\mathrm{m} / \mathrm{z}):[\mathrm{M}+\mathrm{H}]^{+}$calcd for $\mathrm{C}_{19} \mathrm{H}_{31} \mathrm{O}_{4}{ }_{4}, 323.2217$; found, 323.2219.

\section{1-(4-acetylphenyl)-3,3-dimethylbutyl pivalate (10adi)}

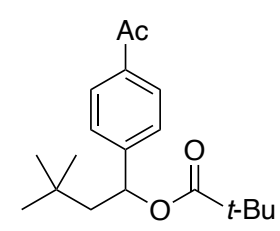

The product 10adi was purified by flash chromatography on silica gel (100:0-7:3, hexane $\left./ \mathrm{CH}_{2} \mathrm{Cl}_{2}\right)$ (Table 2; $15.8 \mathrm{mg}, 0.052 \mathrm{mmol}, 52 \%$ isolated yield). Colorless oil.

IR (neat) 669, 1151, 1267, 1689, 1729, 2340, $2958 \mathrm{~cm}^{-1}$.

${ }^{1} \mathbf{H}$ NMR $\left(600 \mathrm{MHz}, \mathrm{CDCl}_{3}\right) \delta 0.97(\mathrm{~s}, 9 \mathrm{H}), 1.19(\mathrm{~s}, 9 \mathrm{H}), 1.55(\mathrm{dd}, J=2.4,7.2 \mathrm{~Hz}, 1 \mathrm{H}), 1.97$ (dd, $J=9.0,15.0 \mathrm{~Hz}, 1 \mathrm{H}), 2.58(\mathrm{~s}, 3 \mathrm{H}), 5.82(\mathrm{dd}, J=3.0,9.0 \mathrm{~Hz}, 1 \mathrm{H}), 7.38(\mathrm{~d}, J=8.0 \mathrm{~Hz}, 1 \mathrm{H}), 7.92(\mathrm{~d}$, $J=8.0 \mathrm{~Hz}, 2 \mathrm{H})$.

${ }^{13} \mathrm{C}$ NMR (150.9 MHz, $\left.\mathrm{CDCl}_{3}\right) \delta 26.6,27.0$ (3C), 29.9 (3C), 30.5, 38.5, 50.1, 73.5, 125.9 (2C), 128.7 (2C), 136.3, 148.3, 177.5, 197.6.

HRMS-DART $(\mathrm{m} / \mathrm{z})$ : $\left[\mathrm{M}+\mathrm{NH}_{4}\right]^{+}$calcd for $\mathrm{C}_{19} \mathrm{H}_{32} \mathrm{~N}_{1} \mathrm{O}_{3}{ }^{+}, 322.2382$; found, 322.2376 .

\section{1-([1,1'-biphenyl]-4-yl)-3,3-dimethylpentyl pivalate (10bda)}

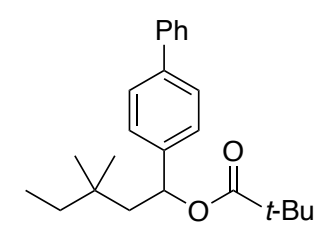

The product 10bda was purified by flash chromatography on silica gel (100:0-10:1, hexane $/ \mathrm{CH}_{2} \mathrm{Cl}_{2}$ ) (Table 2; $14.0 \mathrm{mg}, 0.040 \mathrm{mmol}, 40 \%$ isolated yield). Pale yellow solid.

M.p. : $54-56{ }^{\circ} \mathrm{C}$

IR (neat) 697, 836, 1280, 1487, 1727, 2360, $2961 \mathrm{~cm}^{-1}$.

${ }^{1} \mathbf{H}$ NMR $\left(400 \mathrm{MHz}, \mathrm{CDCl}_{3}\right) \delta 0.85(\mathrm{t}, J=7.6 \mathrm{~Hz}, 3 \mathrm{H}), 0.93(\mathrm{~d}, J=9.2 \mathrm{~Hz}, 6 \mathrm{H}), 1.20$ (s, 9H), $1.23-1.39(\mathrm{~m}, 2 \mathrm{H}), 1.59(\mathrm{dd}, J=2.8,15.2 \mathrm{~Hz}, 1 \mathrm{H}), 2.00(\mathrm{dd}, J=9.2,14.8 \mathrm{~Hz}, 1 \mathrm{H}), 5.84(\mathrm{dd}, J=2.8$, $9.2 \mathrm{~Hz}, 1 \mathrm{H}), 7.31-7.37(\mathrm{~m}, 3 \mathrm{H}), 7.43(\mathrm{t}, J=7.6 \mathrm{~Hz}, 2 \mathrm{H}), 7.44-7.58(\mathrm{~m}, 4 \mathrm{H})$.

${ }^{13}$ C NMR (100.5 MHz, $\left.\mathrm{CDCl}_{3}\right) \delta 8.5,27.0$ (3C), 27.1 (2C), 33.0, 34.7, 38.6, 47.8, 73.5, 126.2, 127.1 (2C), 127.2 (4C), 128.7 (2C), 140.3, 140.8, 142.3, 177.6.

HRMS-DART $(\mathrm{m} / \mathrm{z})$ : [M] $]^{+}$calcd for $\mathrm{C}_{24} \mathrm{H}_{32} \mathrm{O}_{2}{ }^{+}, 352.2402$; found, 352.2407. 


\section{- References}

(1) Sheldrick, G. M. Acta Crystallogr. Sect. A. 2015, 71, 3-8.

(2) Sheldrick, G. M. Acta Crystallogr. Sect. C. 2015, 71, 3-8.

(3) Sumida, Y.; Harada, R.; Kato-Sumida, T.; Johmoto, K.; Uekusa, H.; Hosoya, T. Org. Lett. 2014, $16,6240-6243$.

(4) Numano, M.; Nagami, N.; Nakatsuka, S.; Katayama, T.; Nakajima, K.; Tatsumi, S.; Yasuda, N.; Hatakeyama, T. Chem. Eur. J. 2016, 22, 11574-11577.

(5) Hill, R. R.; Rychnovsky, S. D. J. Org. Chem. 2016, 81, 10707-10714.

(6) Huang, N.; Liao, H.; Yao, H.; Xie, T.; Zhang, S.; Zou, K.; Liu, X.-W. Org. Lett. 2018, 20, $16-19$.

(7) Guo, W.-D.; Liu, L.; Yang, S.-Q.; Chen, X.-C.; Lu, Y.; VO-Thanh, G.; Liu, Y. ChemCatChem 2020, 12, 1376-1384.

(8) Liu, J.; Dong, K.; Franke, R.; Neumann, H.; Jackstell, R.; Beller, M. J. Am. Chem. Soc. 2018, 140, 10282-10288.

(9) Lohre, C.; Dröge, T.; Wang, C.; Glorius, F. Chem. - Eur. J. 2011, 17, 6052-6055.

(10) Murphy, J. A.; Garnier, J.; Park, S. R.; Schoenebeck, F.; Zhou, S.-z.; Turner, A. T. Org. Lett. 2008, 10, 1227-1230.

(11) Yang, S.; Chen, M.; Tang, P. Angew. Chem. Int. Ed. 2019, 58, 7840-7844.

(12) Primer, D. N.; Molander, G. A. J. Am. Chem. Soc. 2017, 139, 9847-9850.

(13) Hirashima, S.-i.; Nobuta, T.; Tada, N.; Miura, T.; Itoh, A. Org. Lett. 2010, 12, 3645-3647.

(14) Chow, W. K.; So, C. M.; Lau, C. P.; Kwong, F. Y. Chem. - Eur. J. 2011, 17, 6913-6917.

(15) Delcamp, J. H.; White, M. C. J. Am. Chem. Soc. 2006, 128, 15076-15077.

(16) Clausen, F.; Kischkewitz, M.; Bergander, K.; Studer, A. Chem. Sci. 2019, 10, 6210-6214.

(17) Cornella, J.; Edwards, J. T.; Qin, T.; Kawamura, S.; Wang, J. Pan, C-M.; Gianatassio, R.; Schmidt, M. A.; Eastgate, M. D.; Baran, P. S. J. Am. Chem. Soc. 2016, 138, 2174-2177

(18) Ines, B.; Moreno, I.; SanMartin, R.; Dominguez, E. J. Org. Chem. 2008, 73, 8448-8451.

(19) Shen, Z.-L.; Goh, K. K. K.; Yang, Y.-S.; Lai, Y.-C.; Wong, C. H. A.; Cheong, H.-L.; Loh, T.-P. Angew. Chem. Int. Ed. 2011, 50, 511-514.

(20) Meng, S.-S.; Lin, L.-R.; Luo, X.; Lv, H.-J.; Zhao, J.-L.; Chan, A. S. C. Green Chem. 2019, 21, 6187-6193.

(21) Haydl, A. M.; Hartwig, J. F. Org. Lett. 2019, 21, 1337-1341.

(22) Oyamada, J.; Kitamura, T. Tetrahedron 2006, 62, 6918-6925.

(23) Campbell, M. W.; Compton, J. S.; Kelly, C. B.; Molander, G. A. J. Am. Chem. Soc. 2019, 141, 20069-20078. 


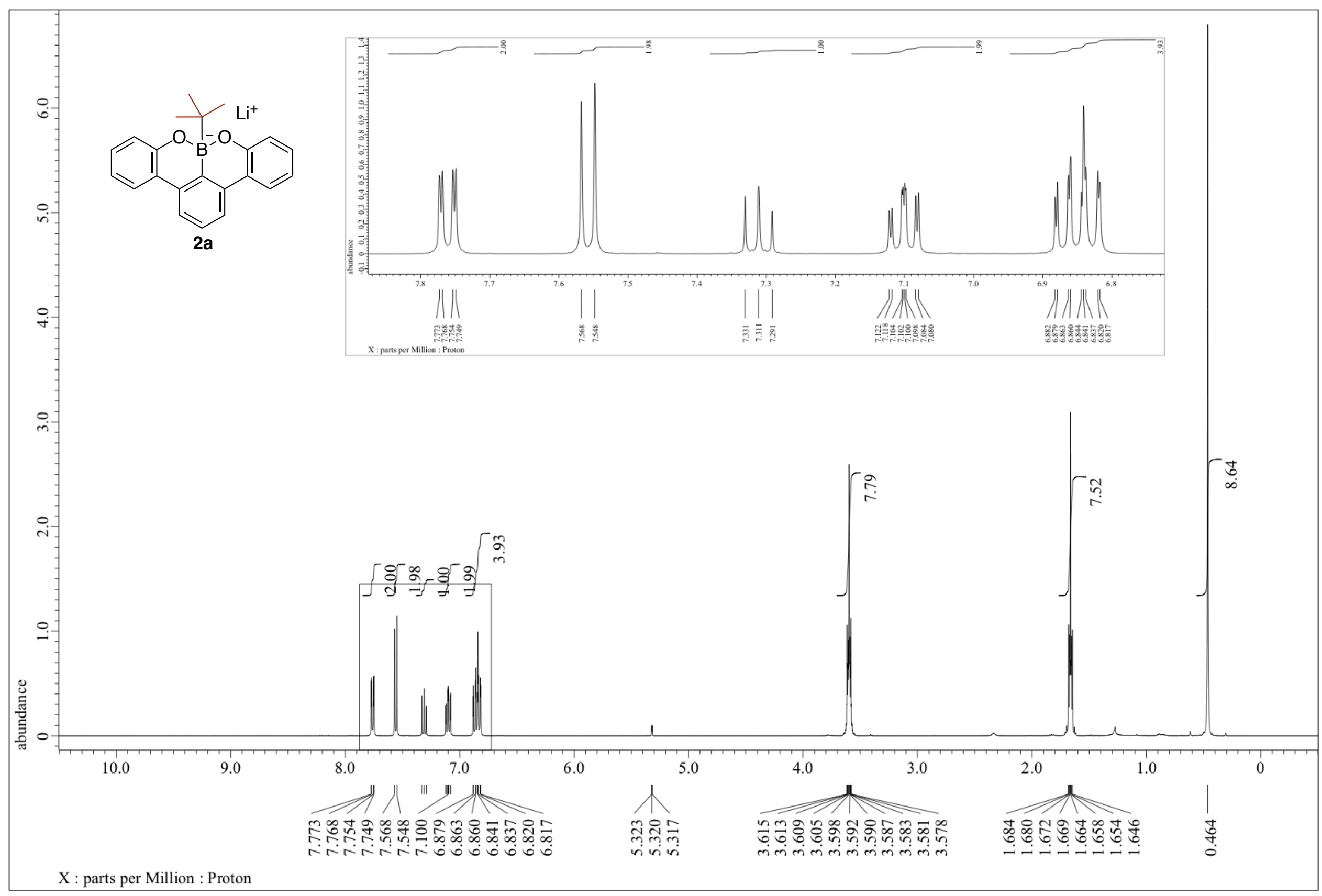




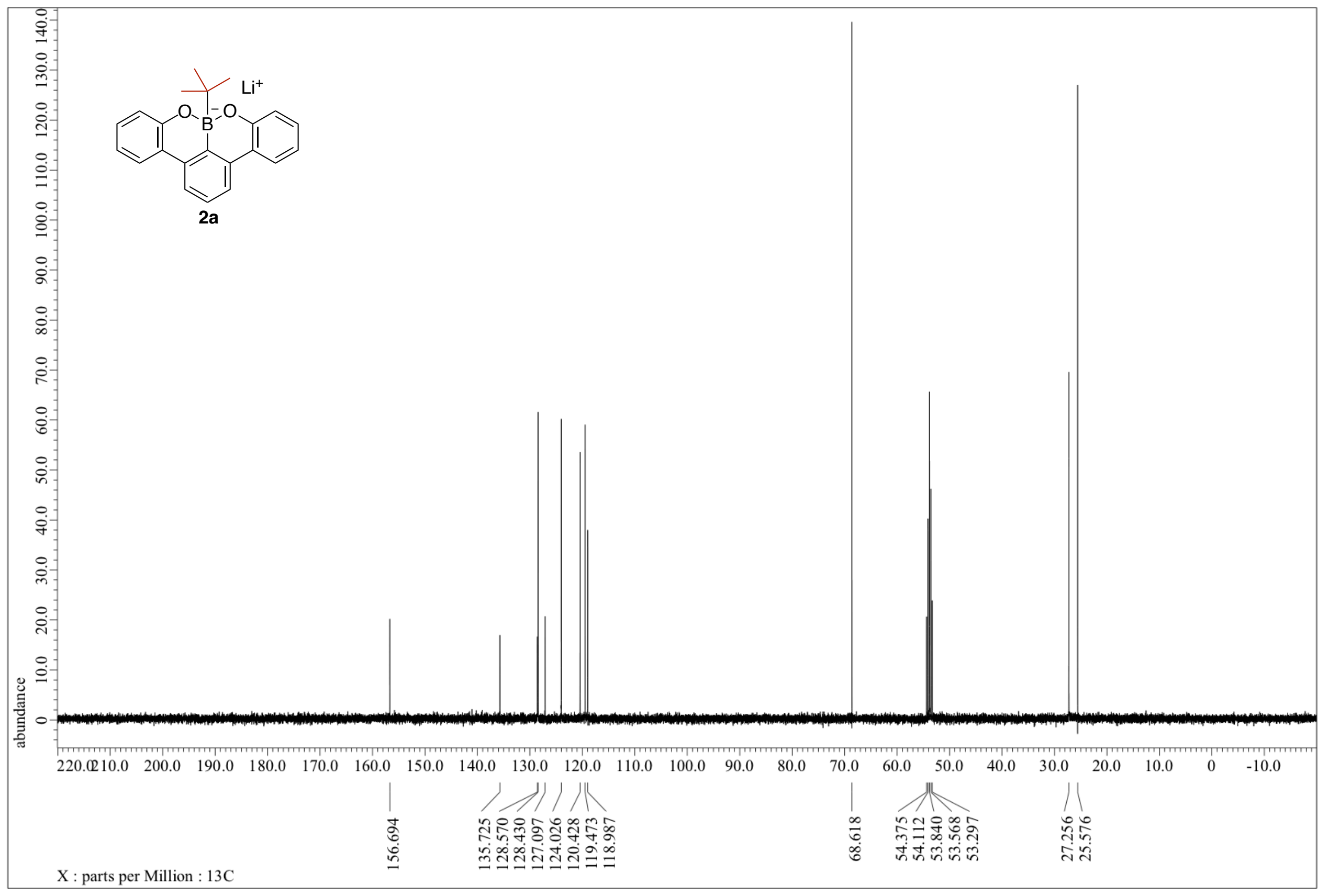




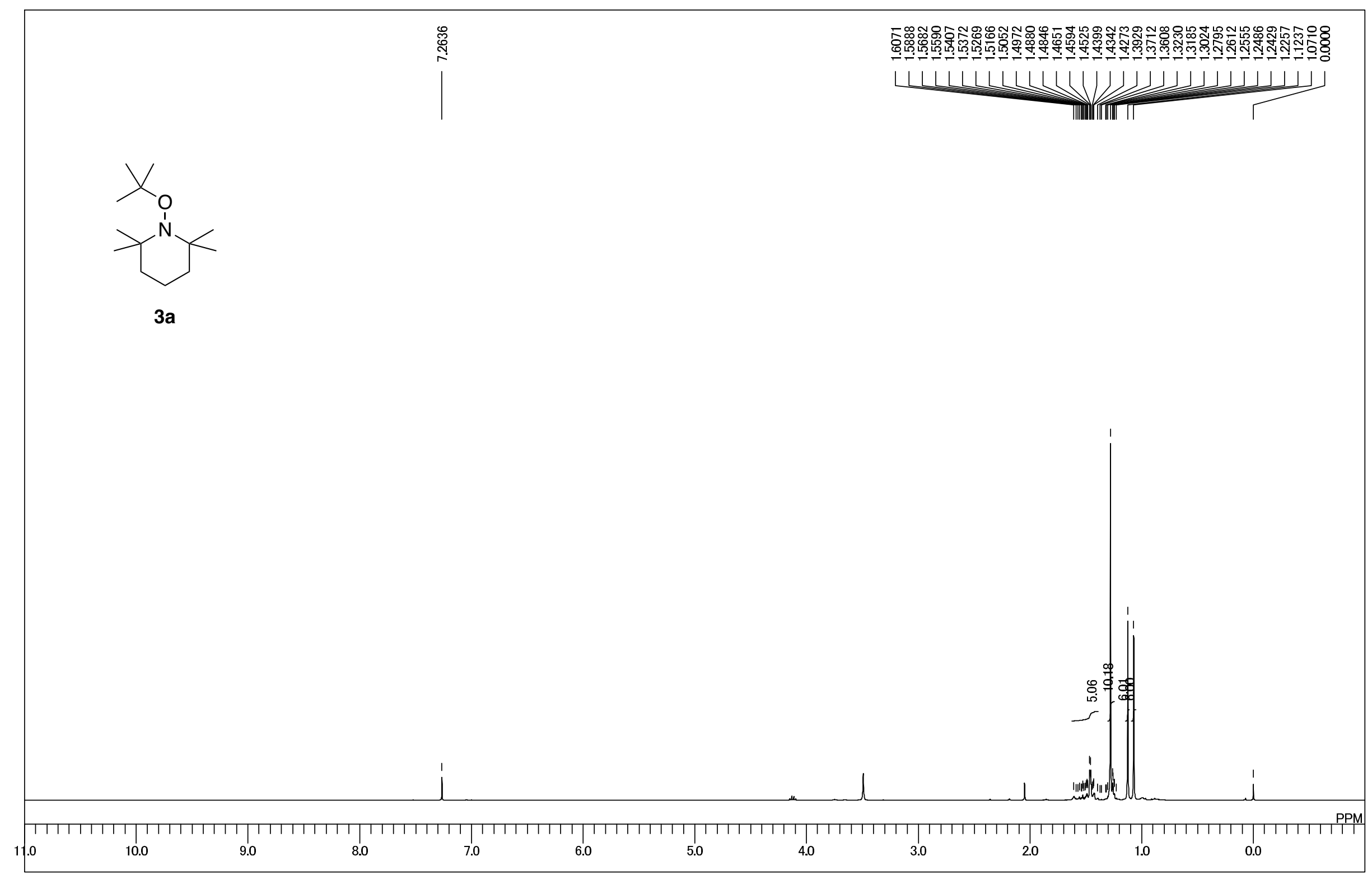




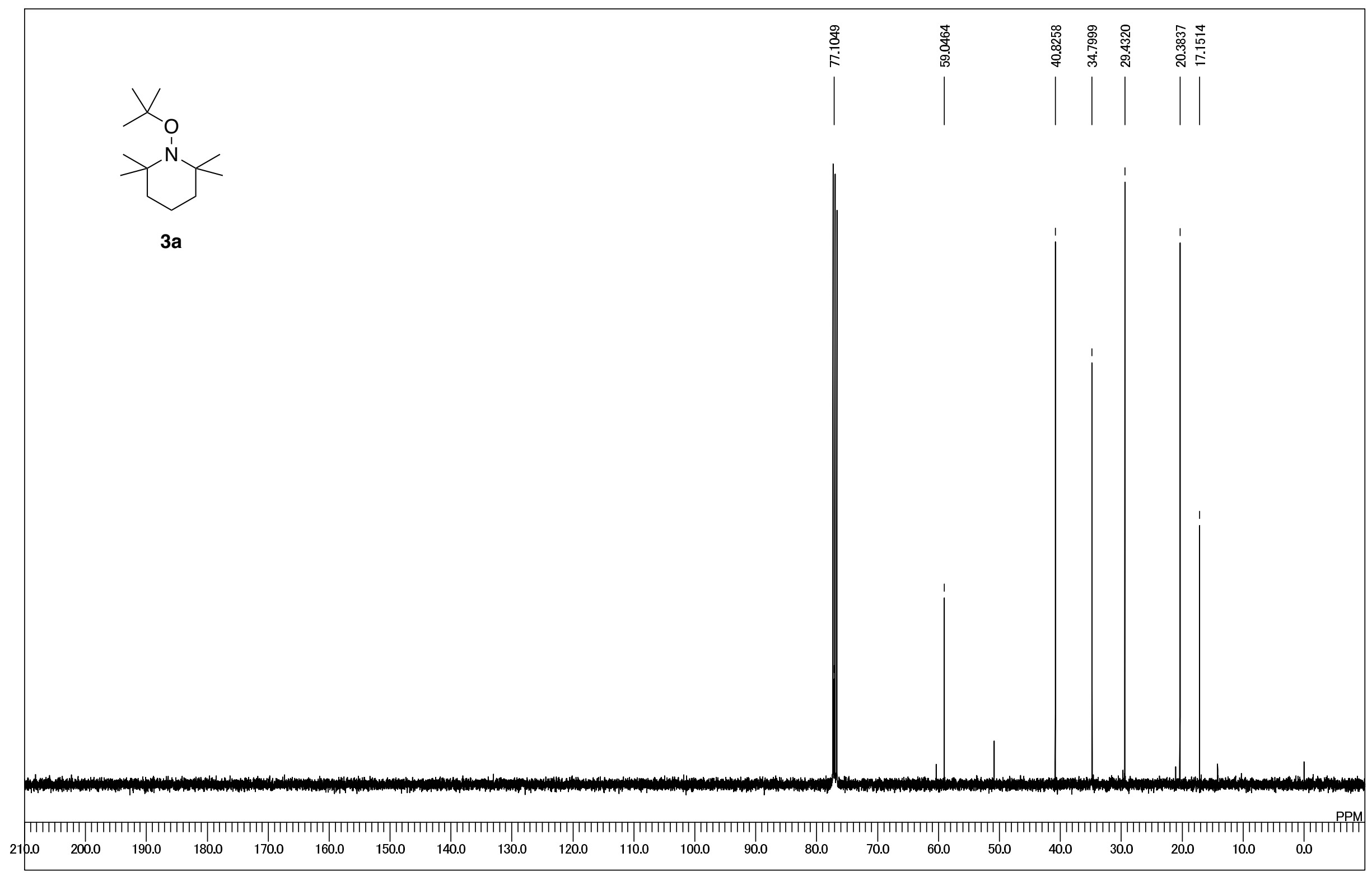




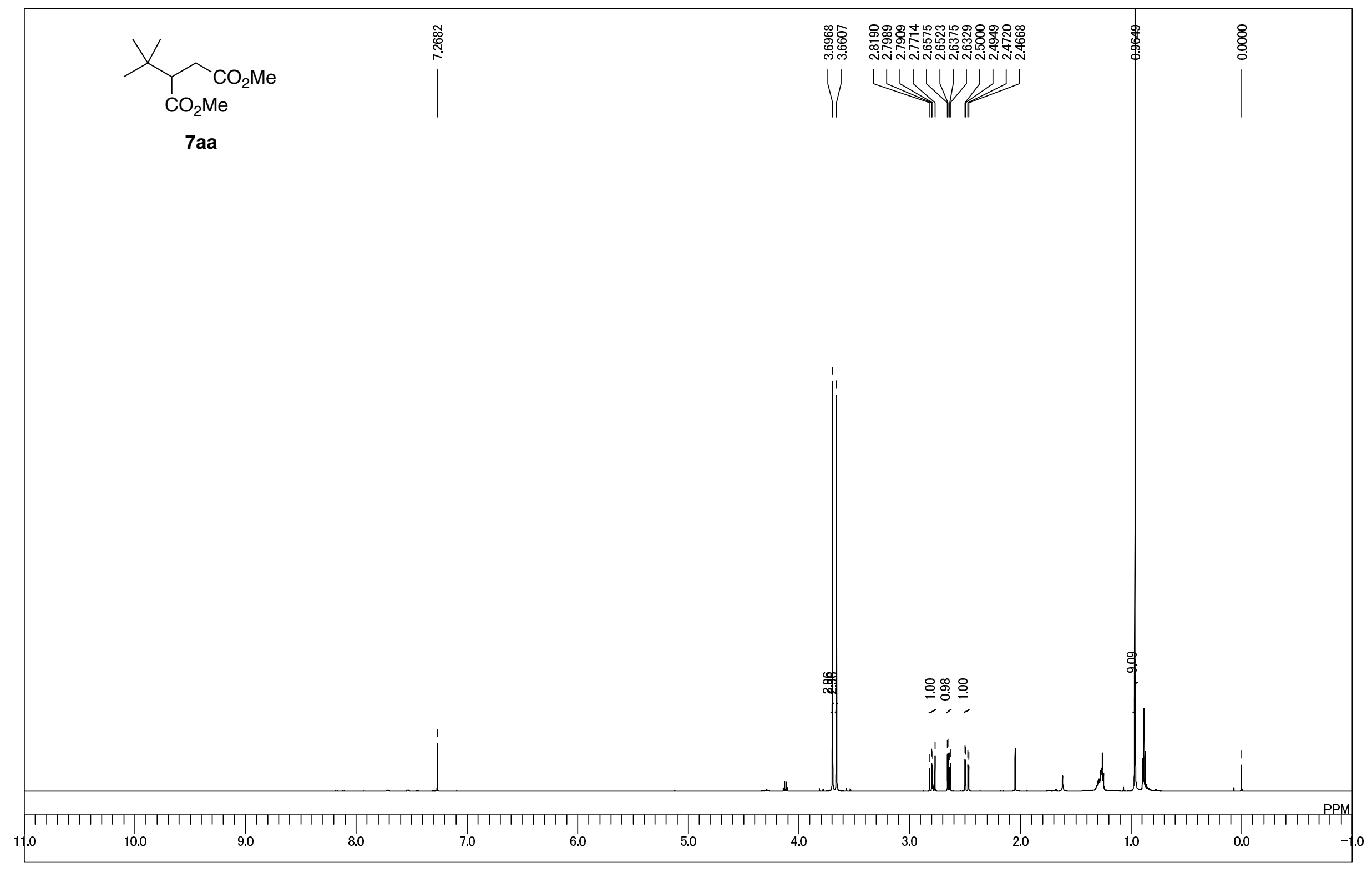




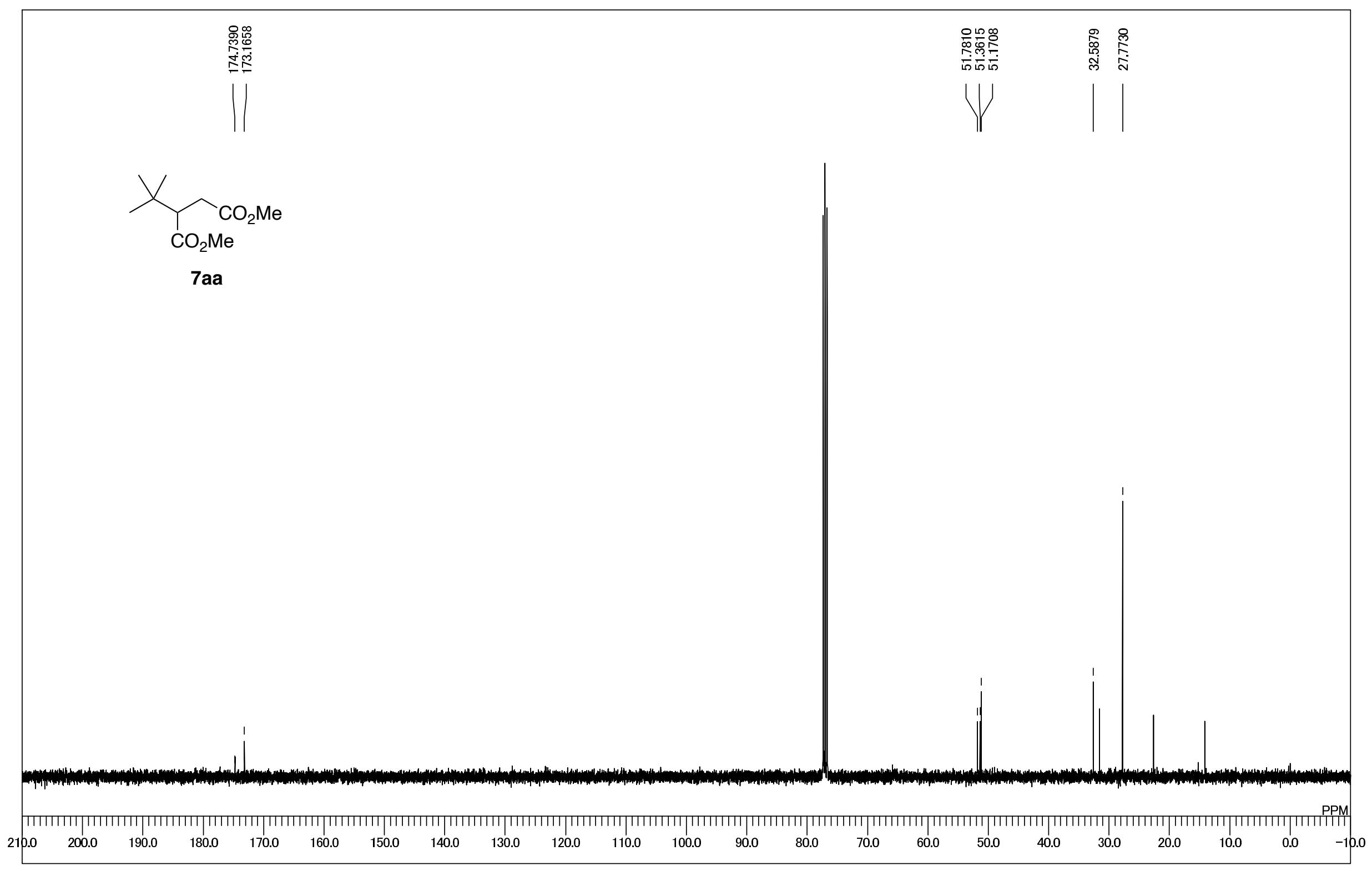




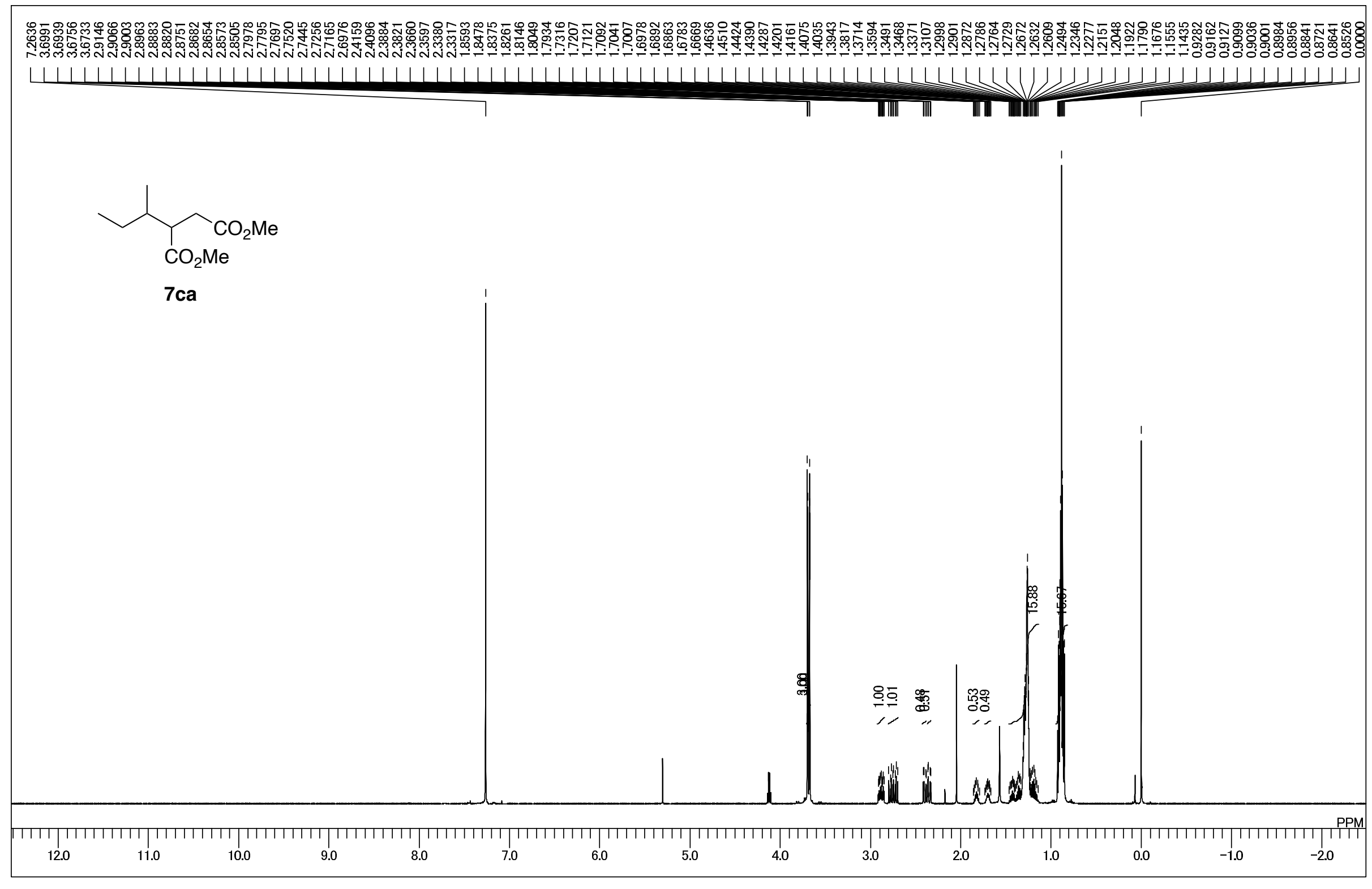




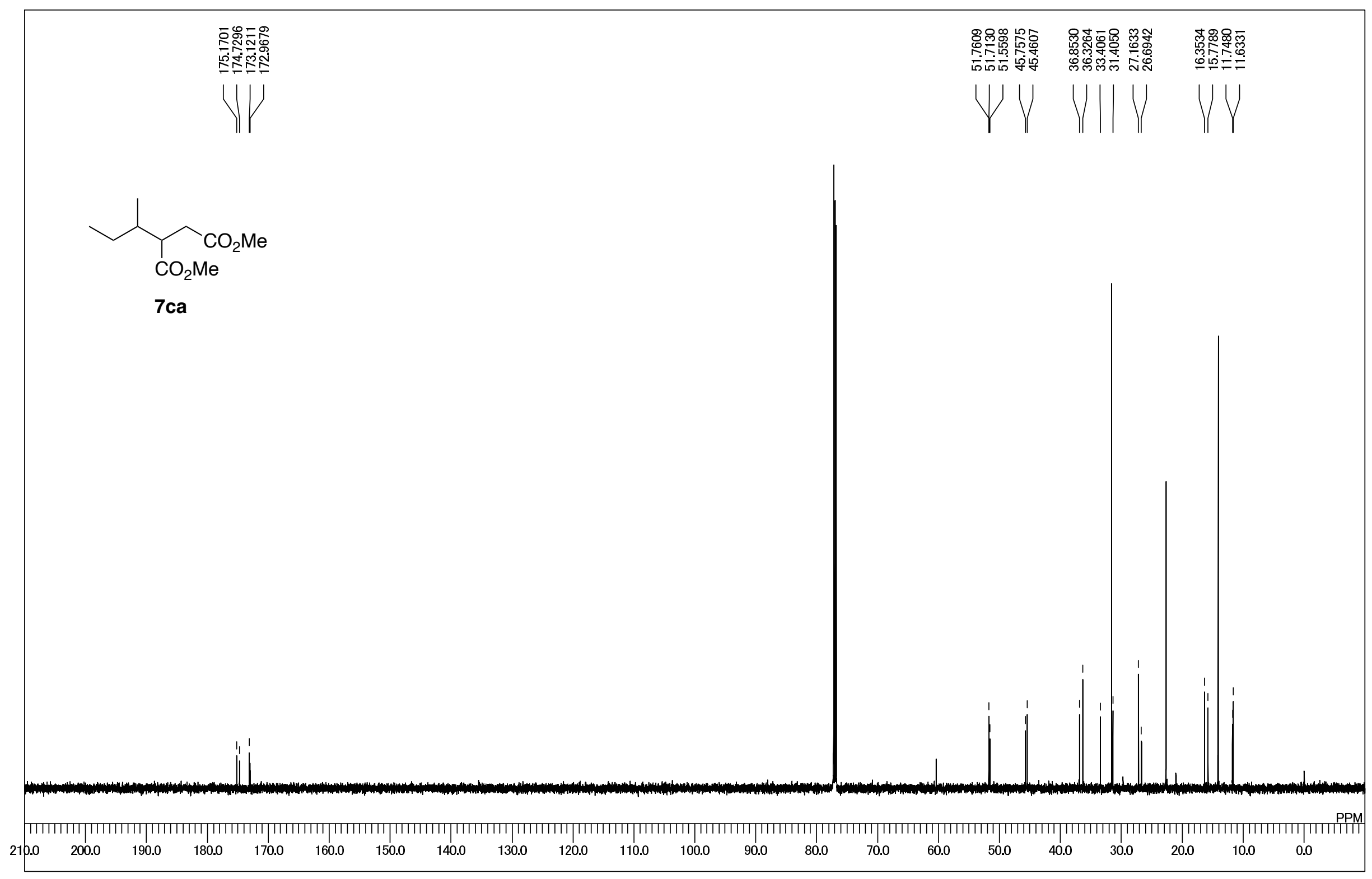




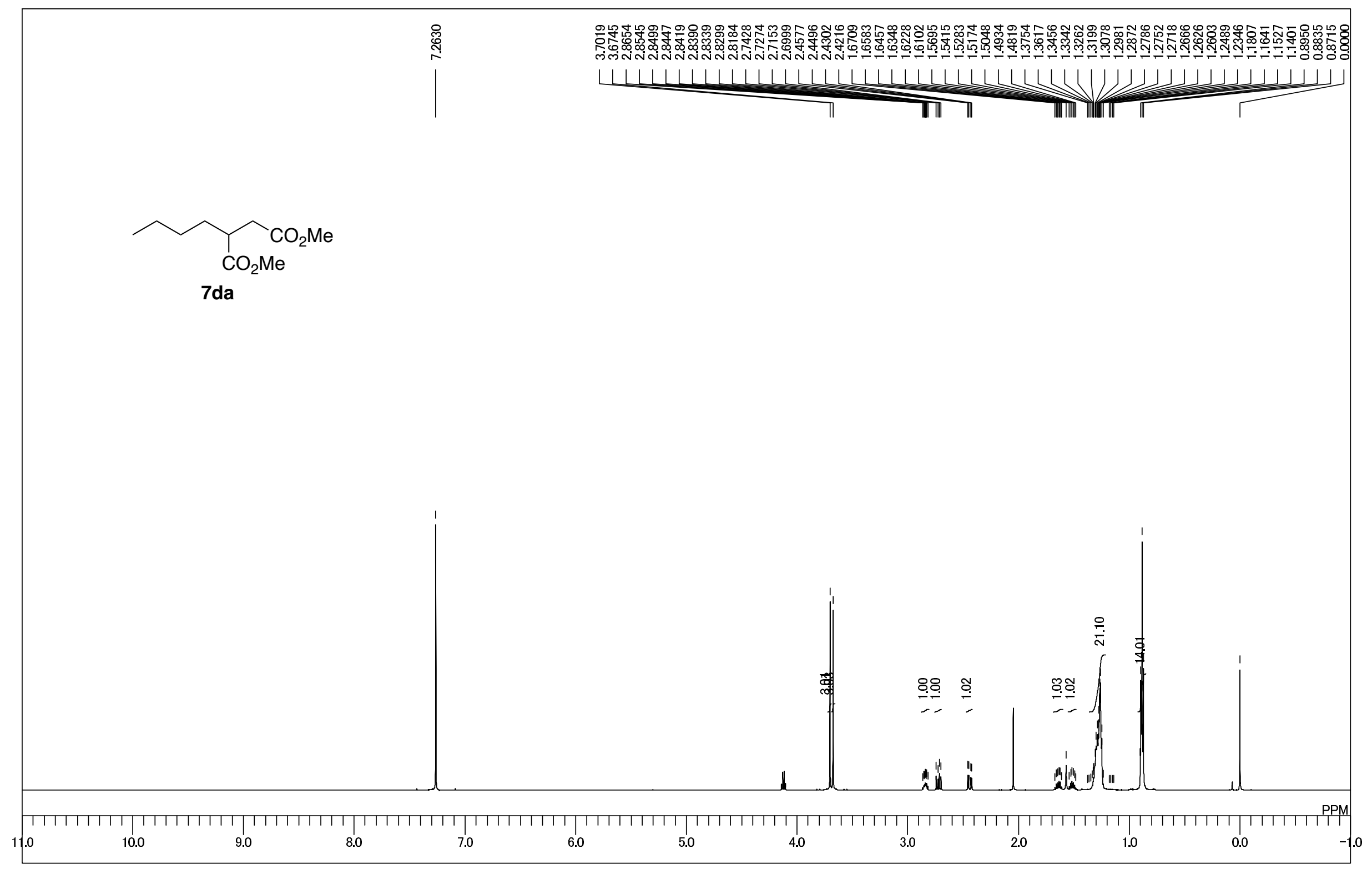




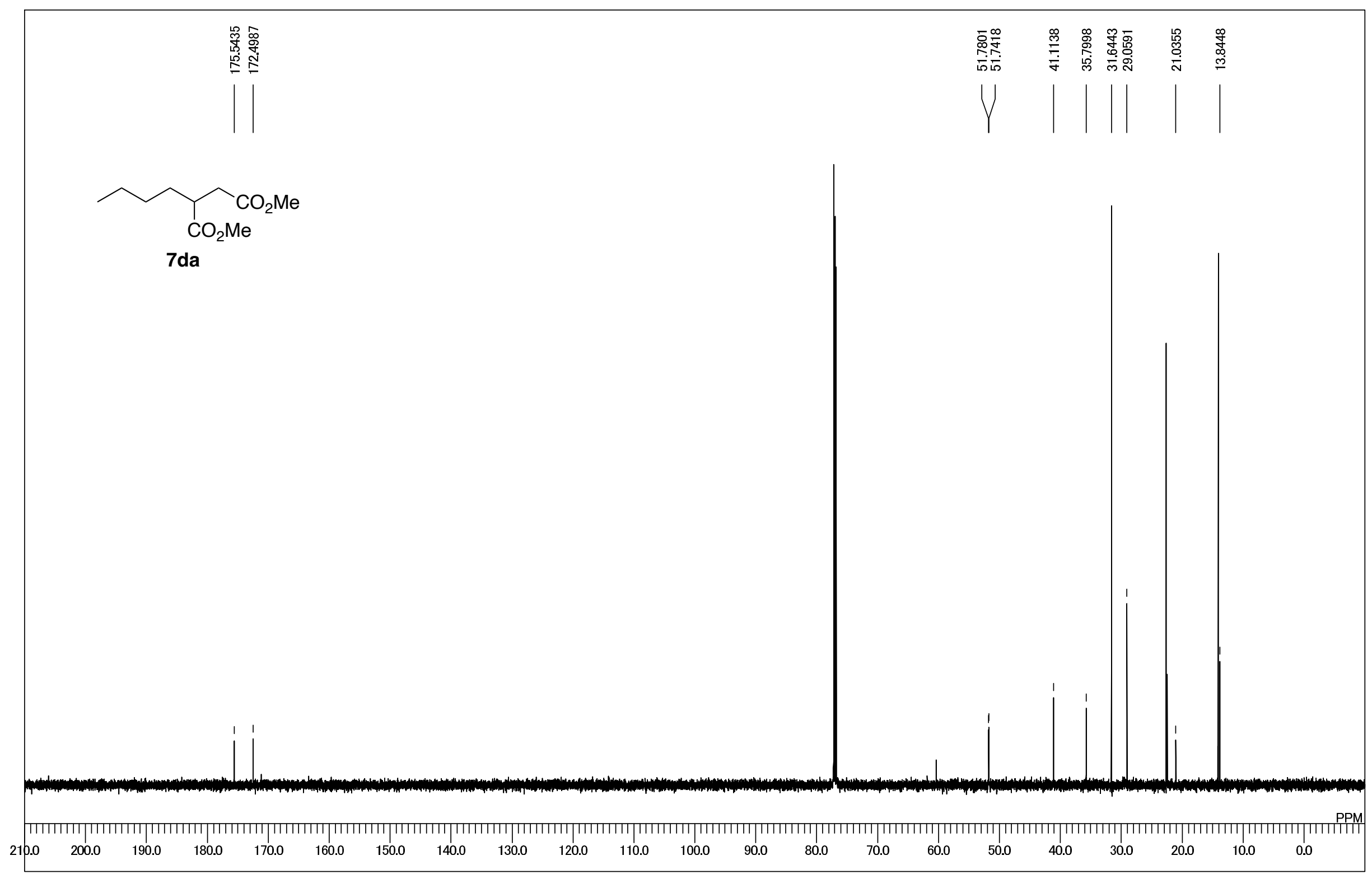




$$
=1
$$




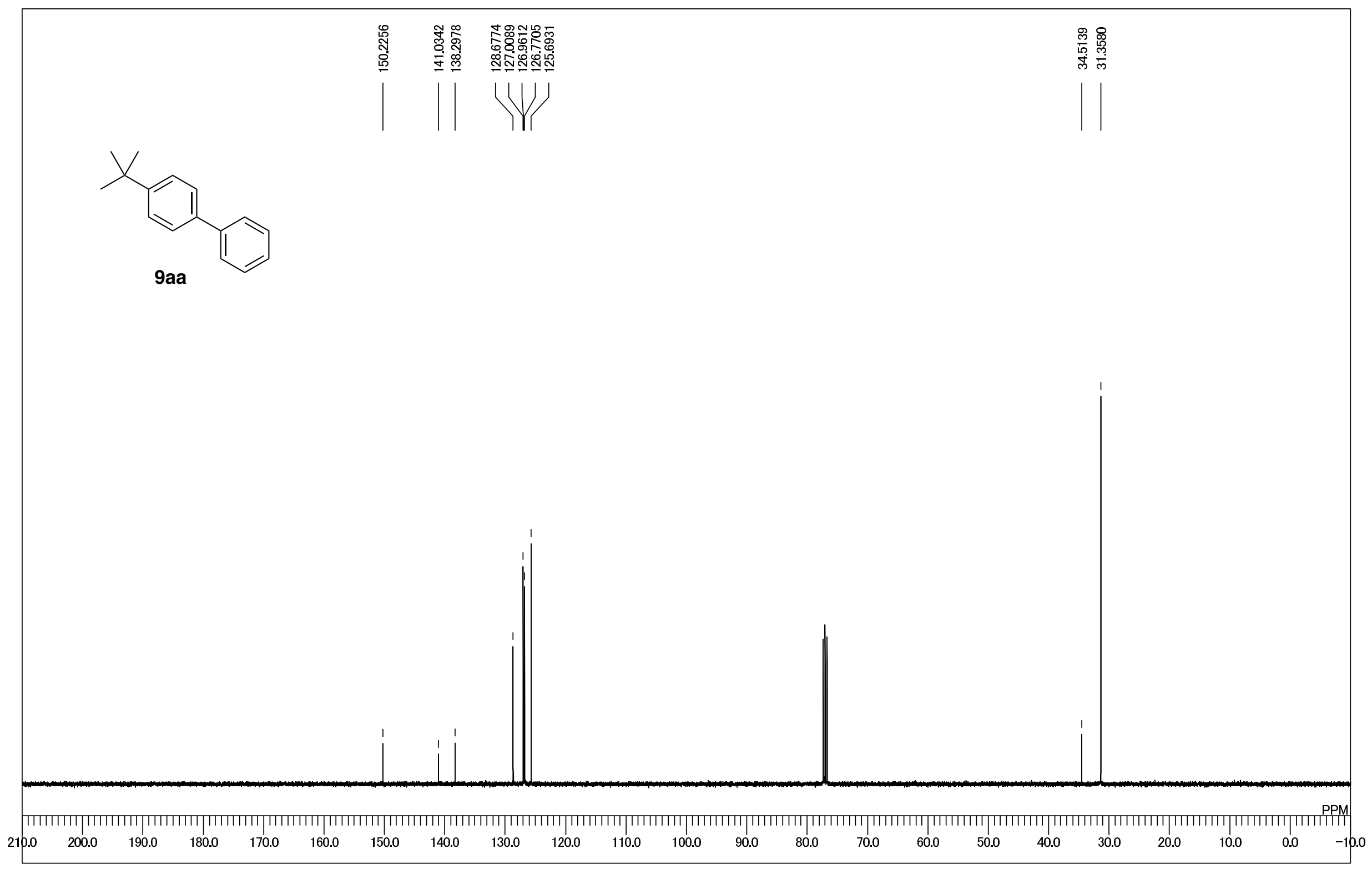




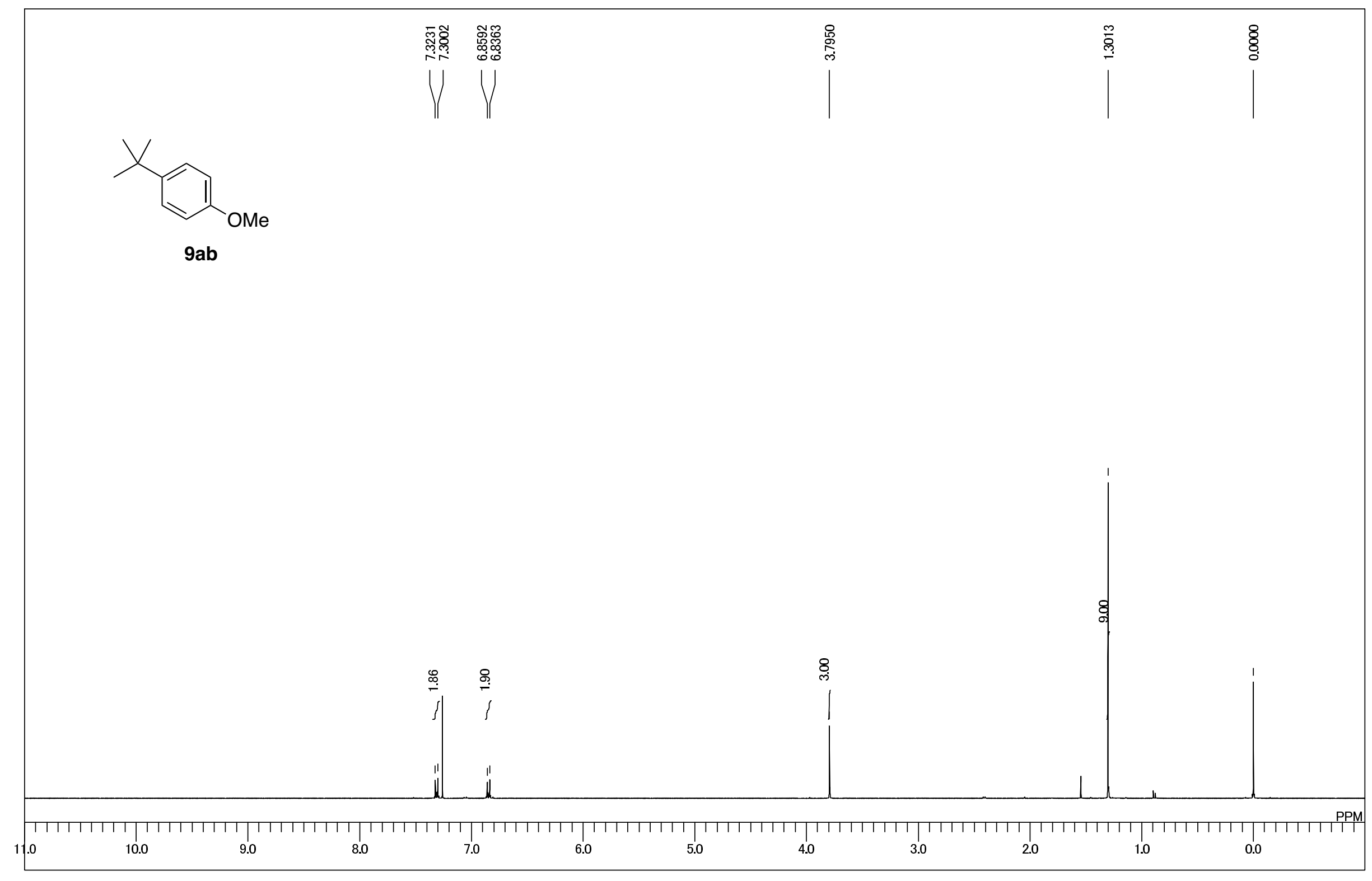




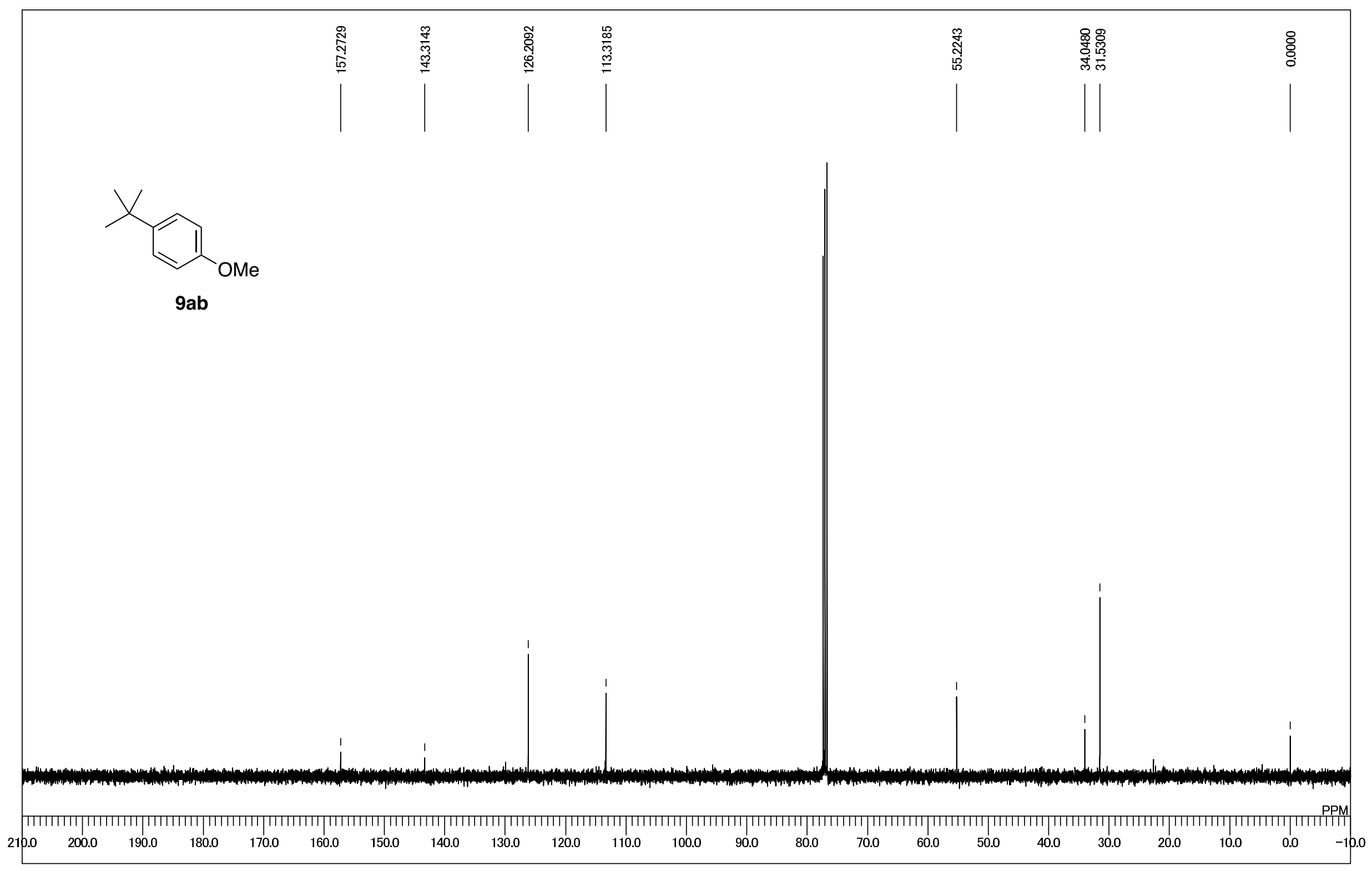




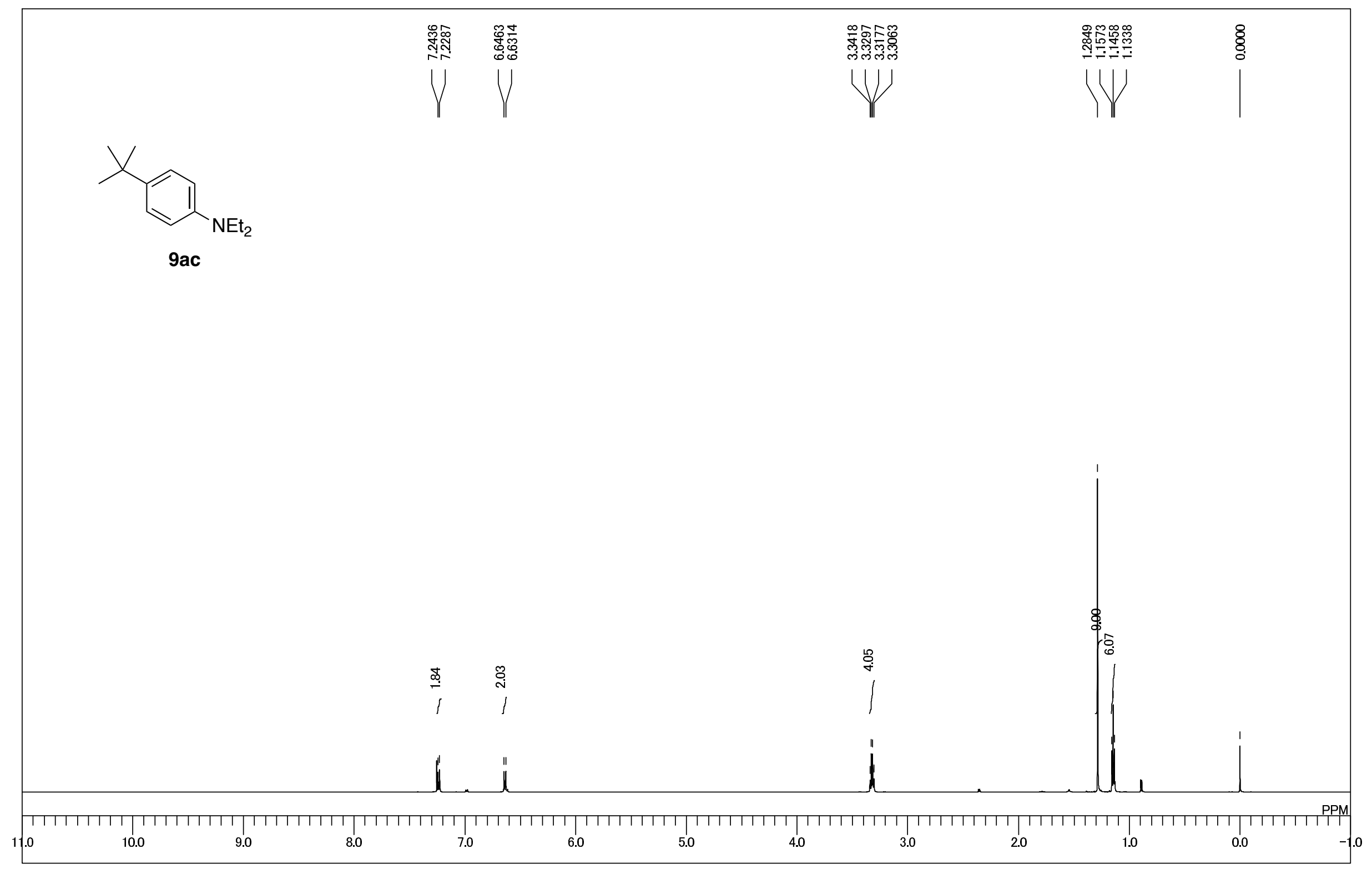




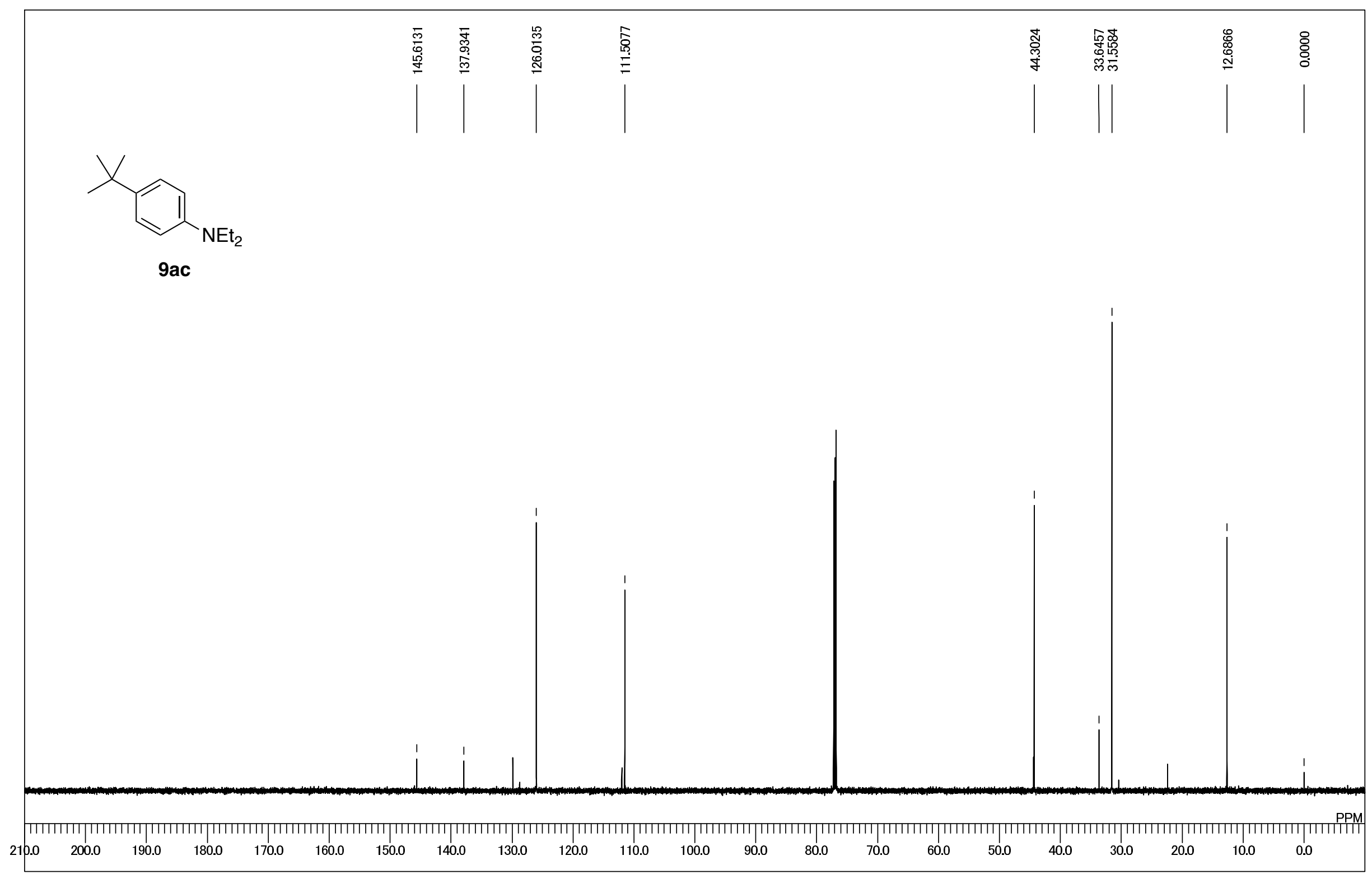




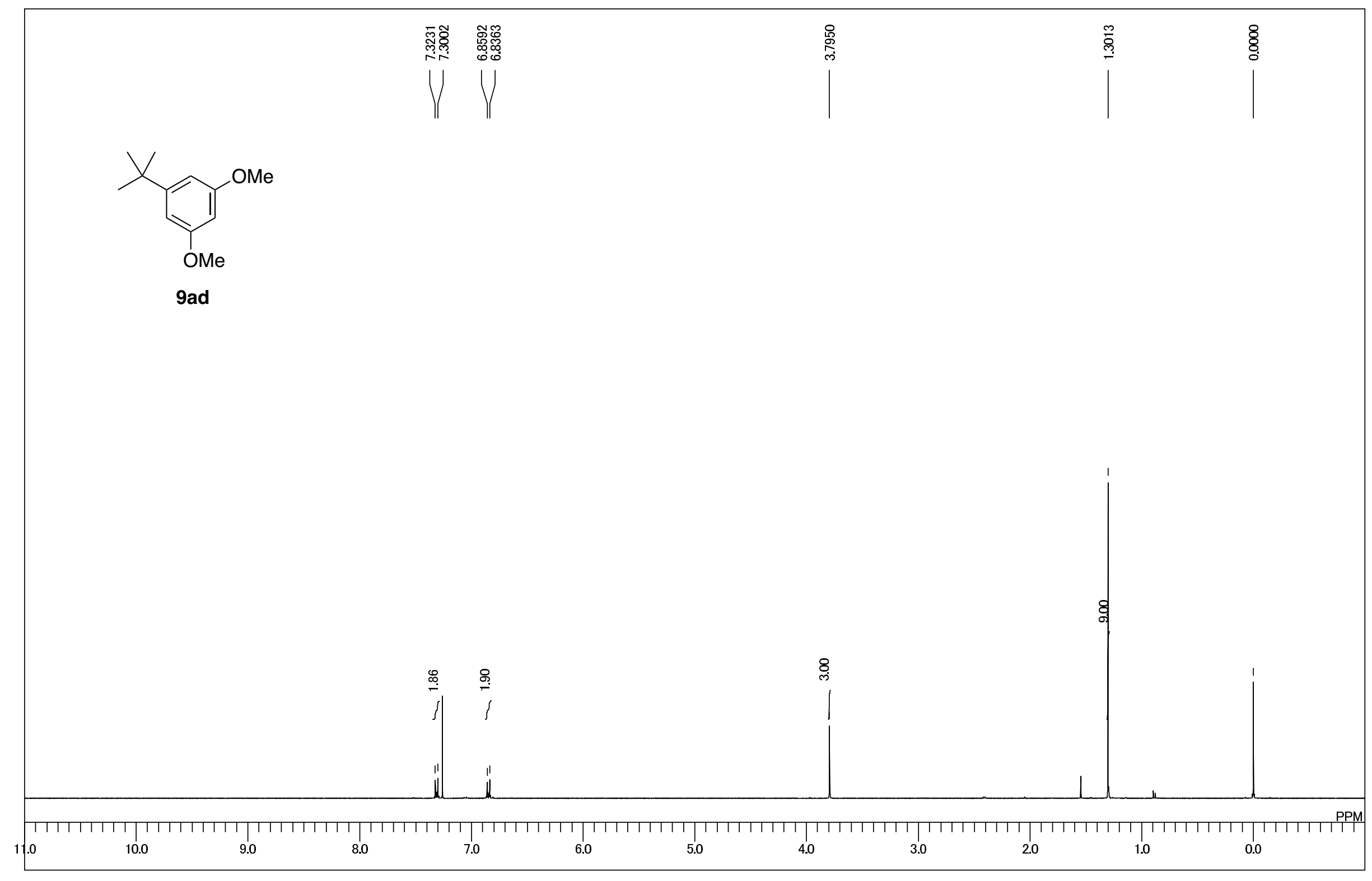




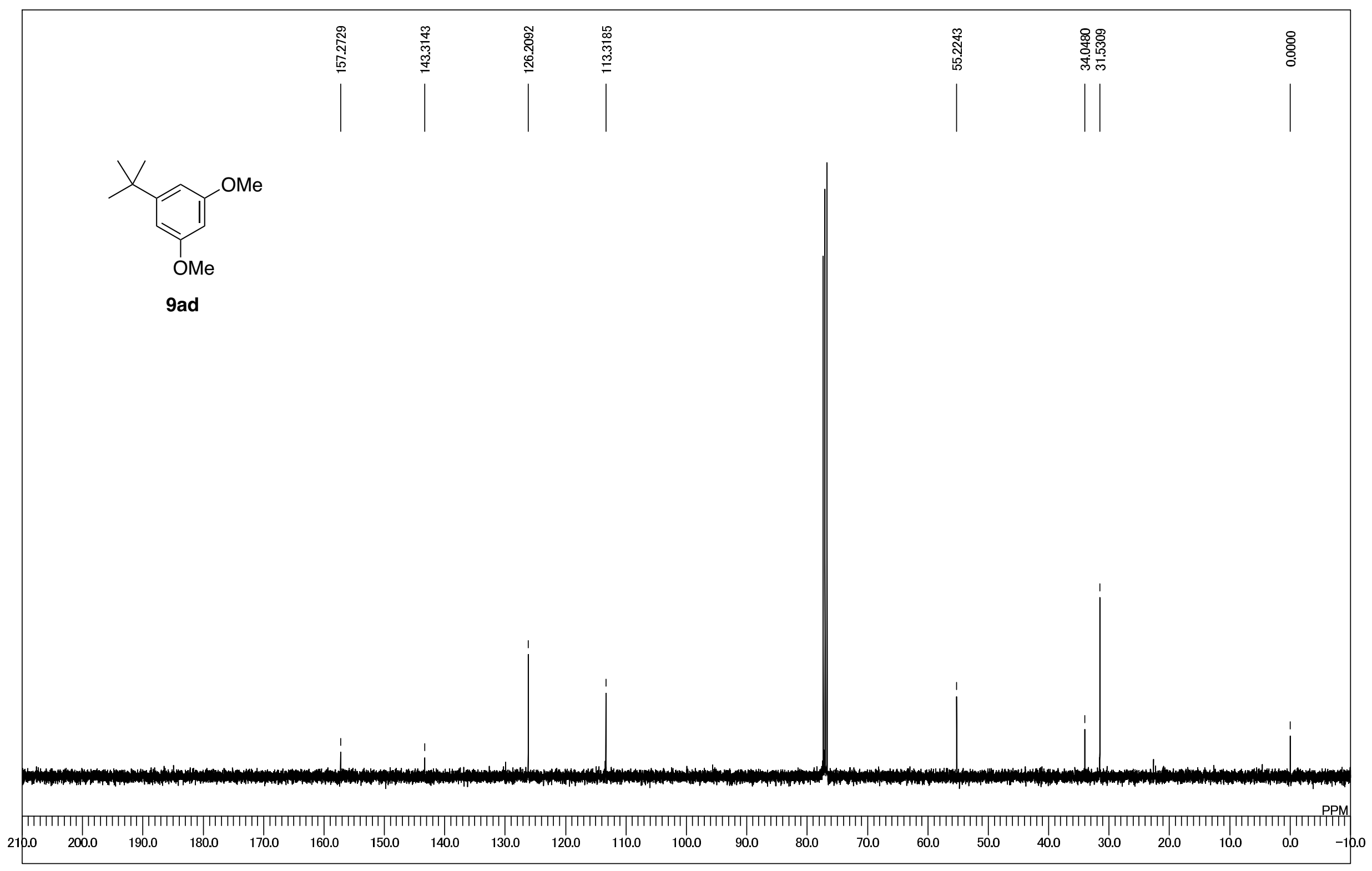




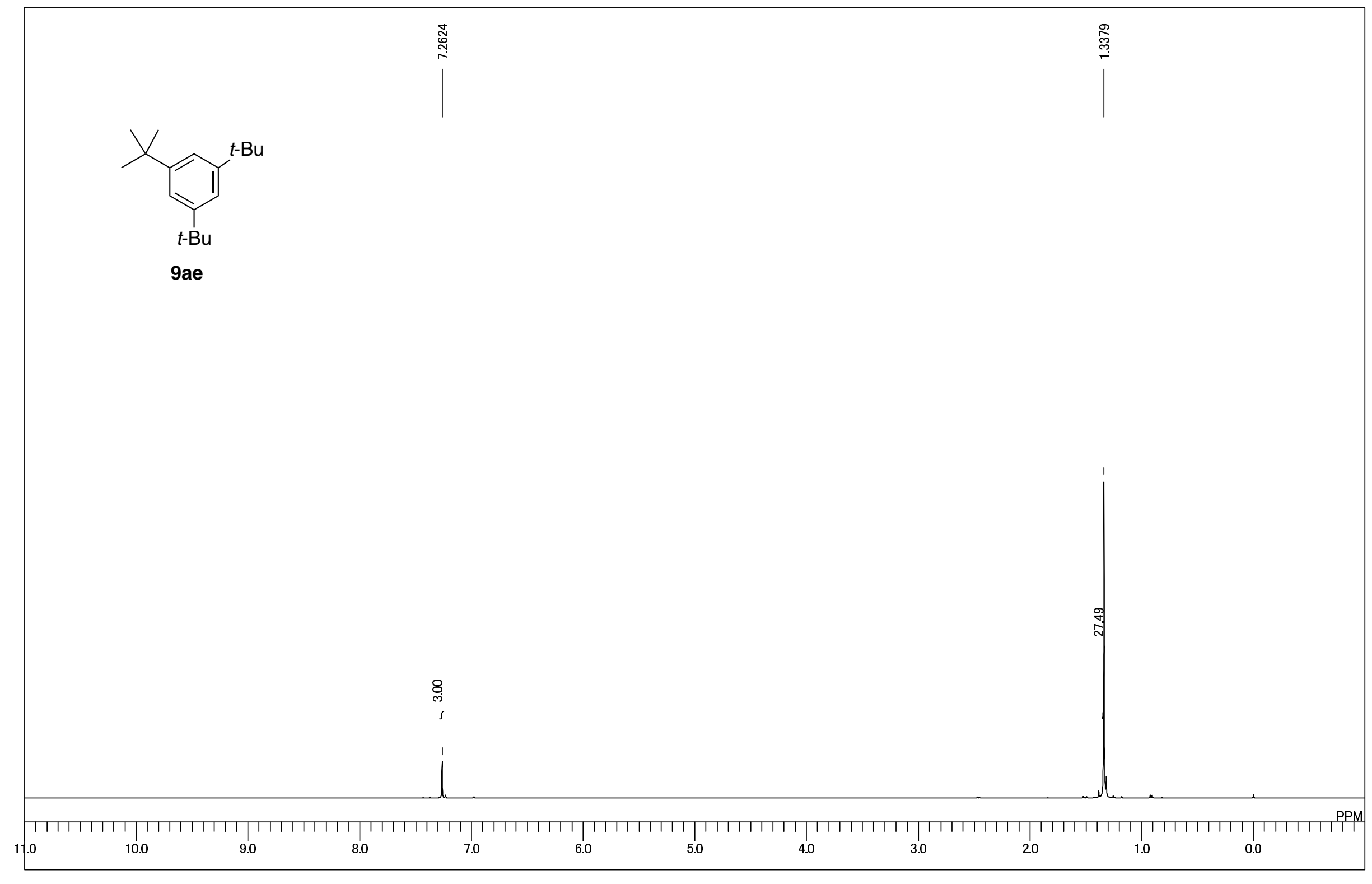




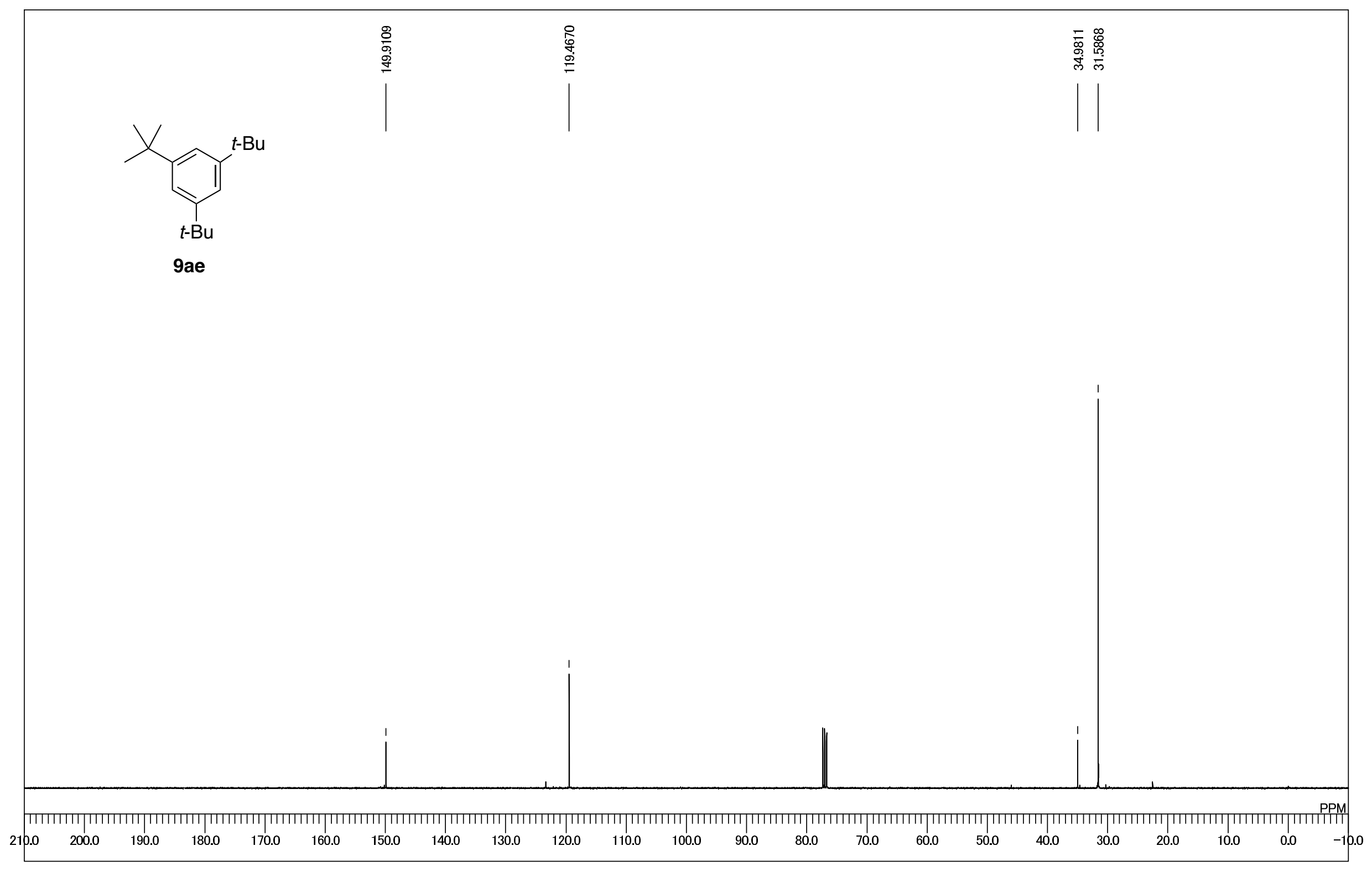




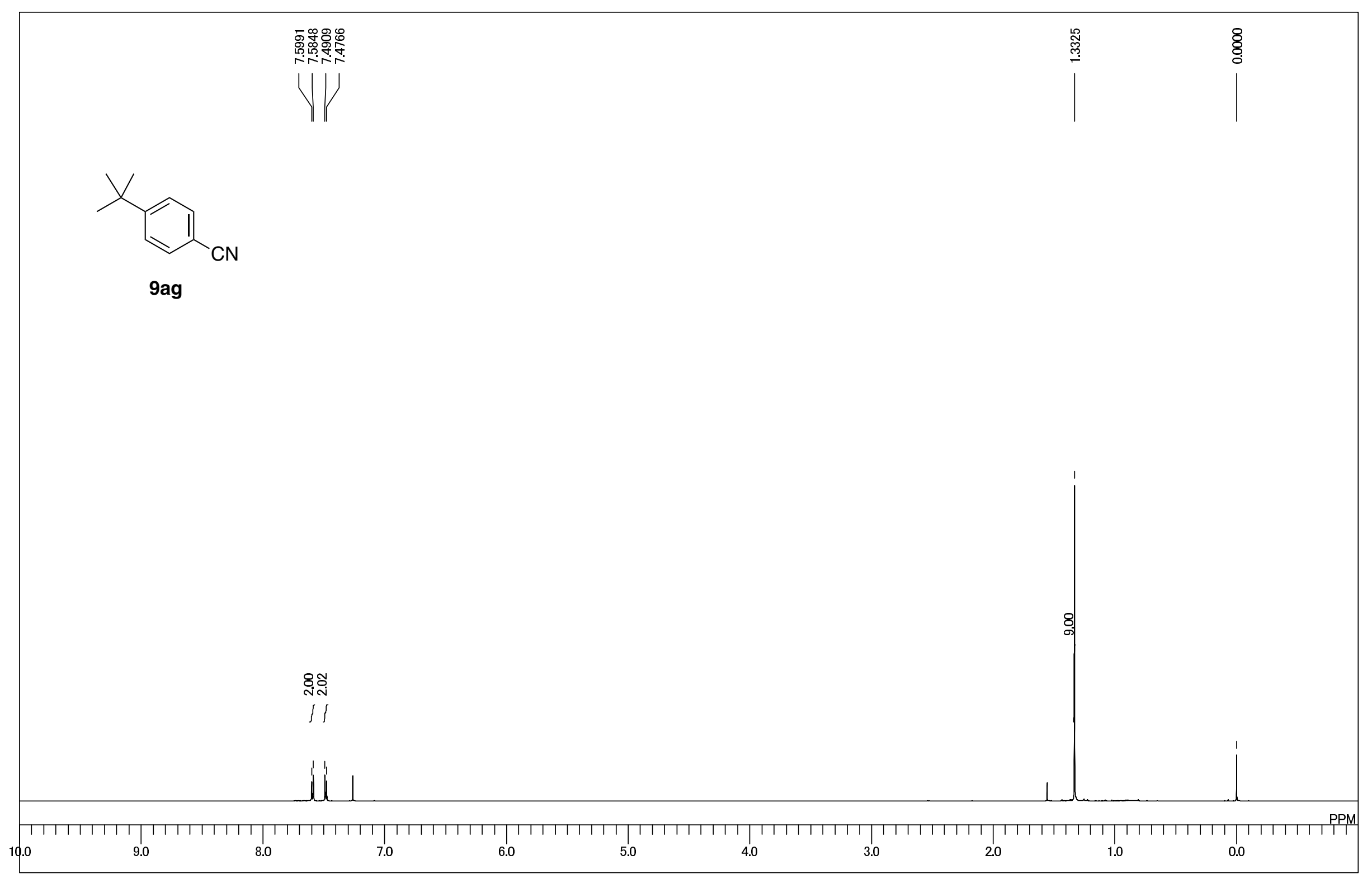

S53 


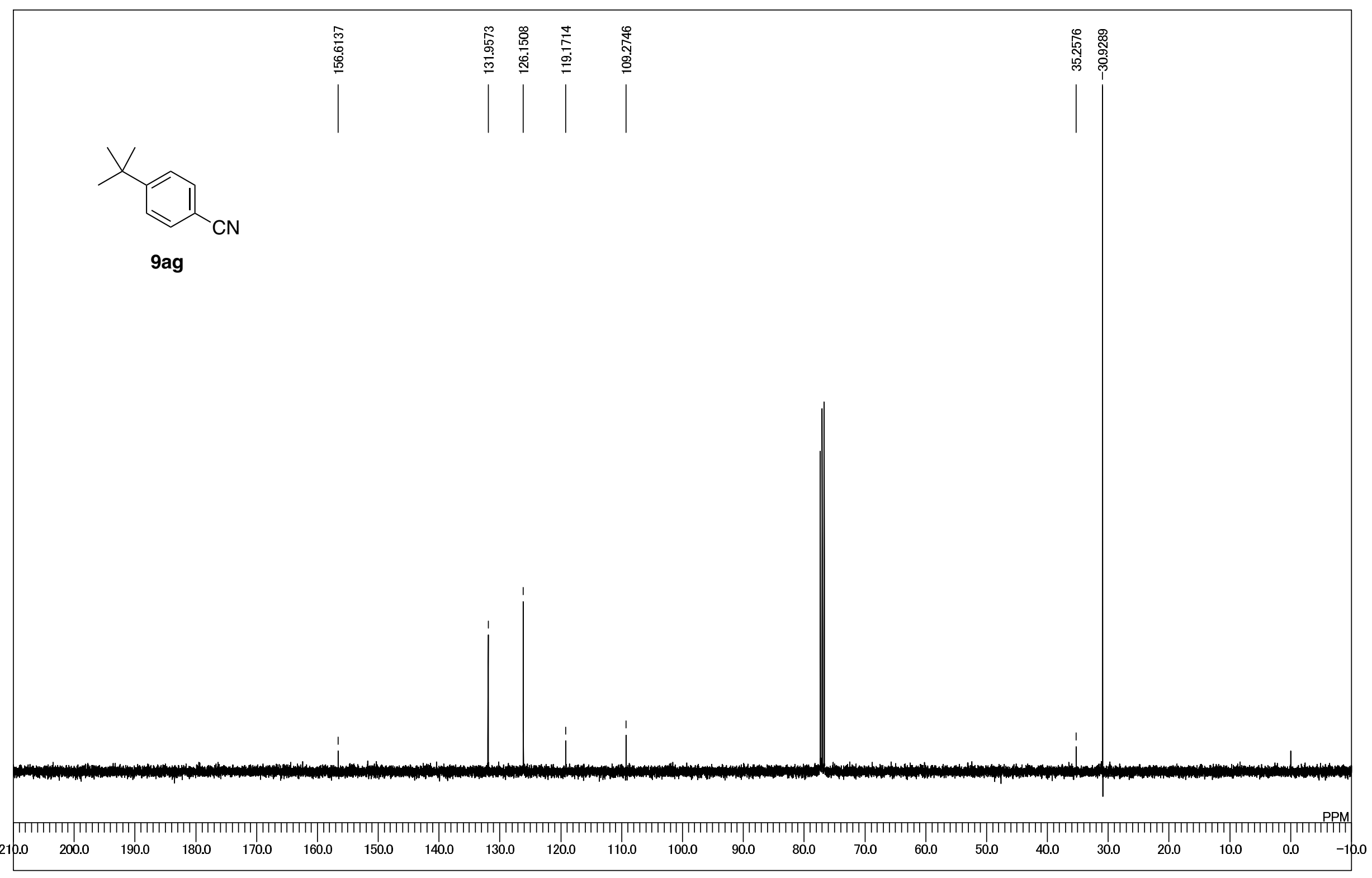




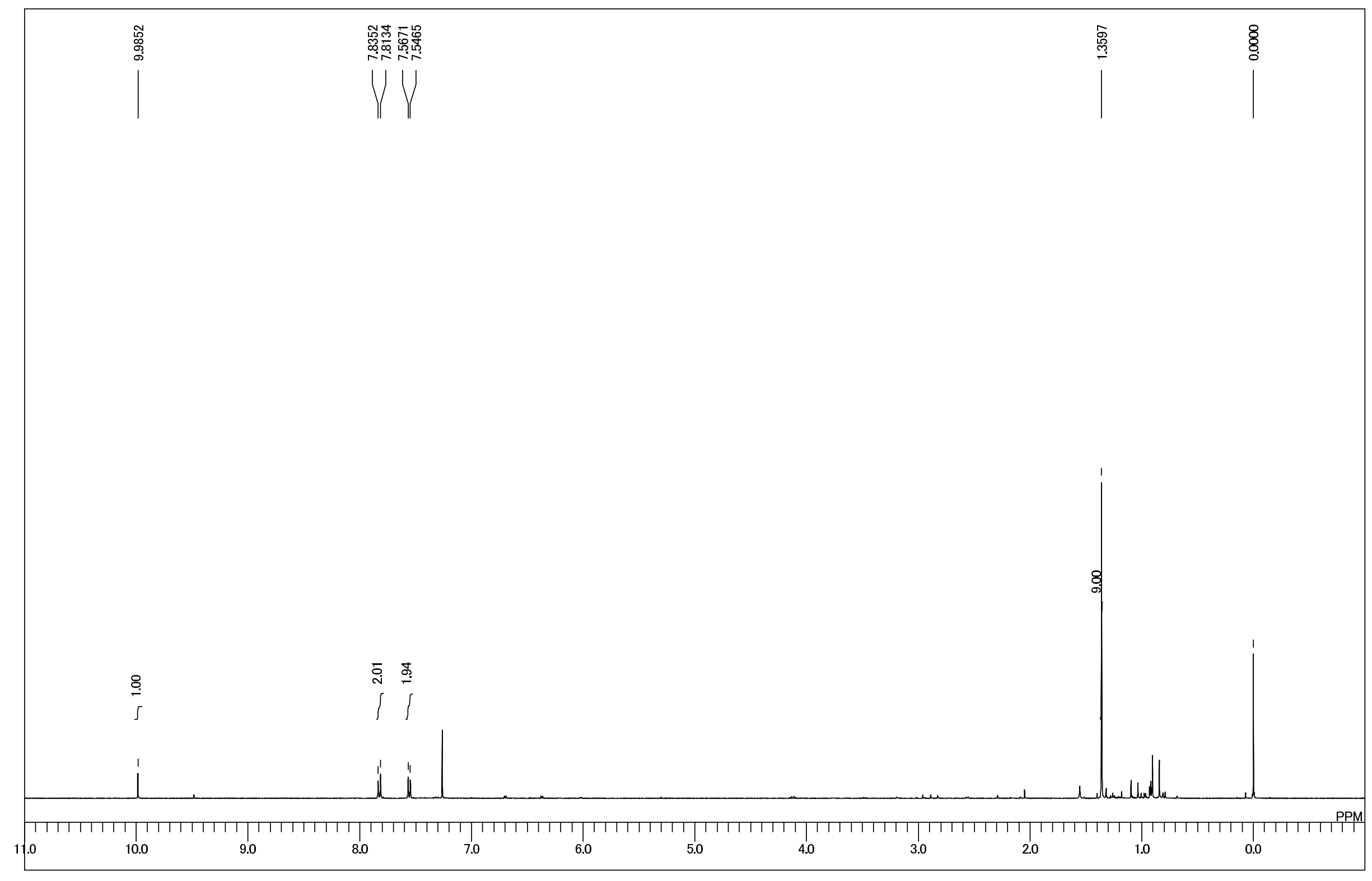




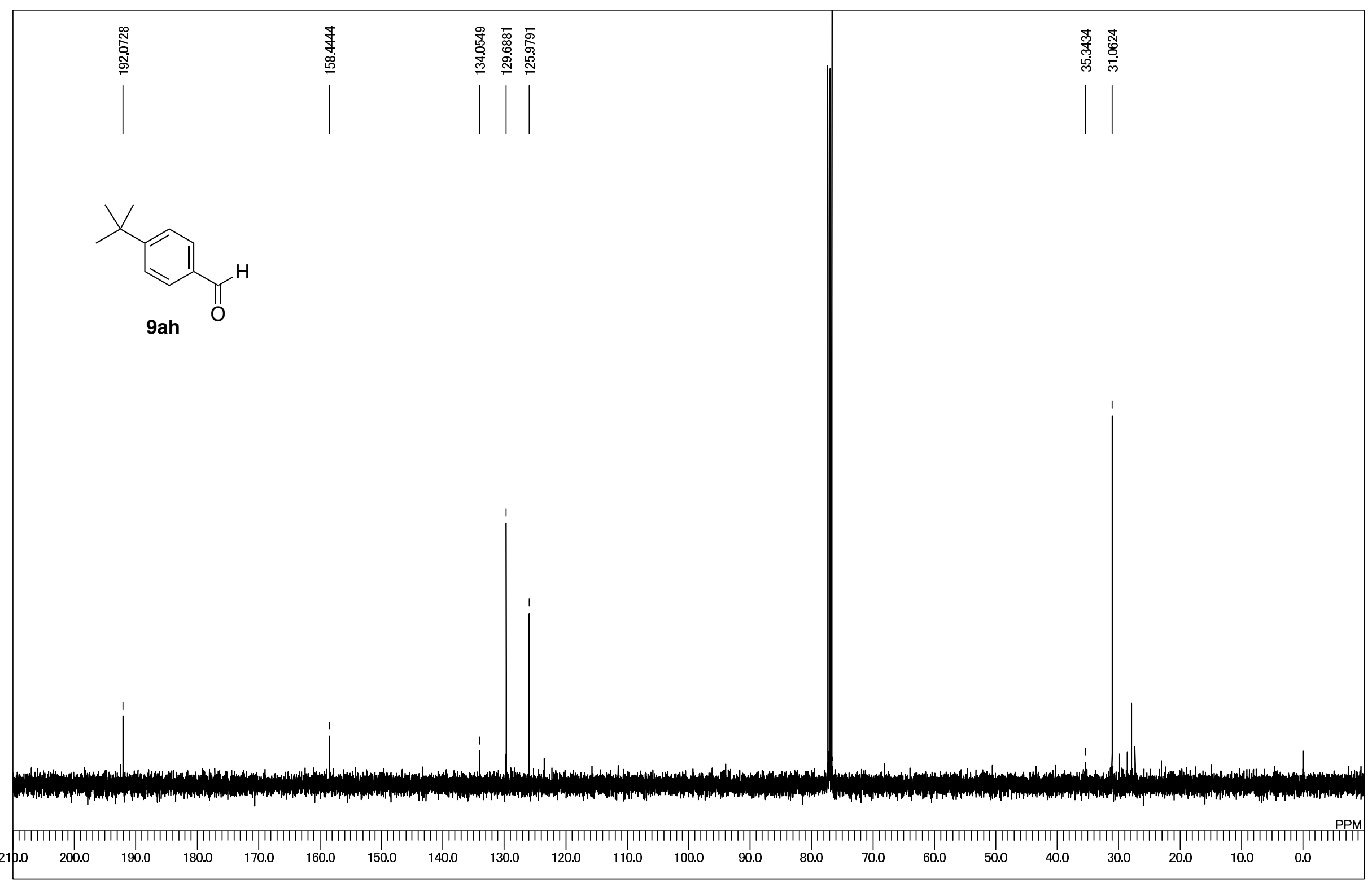




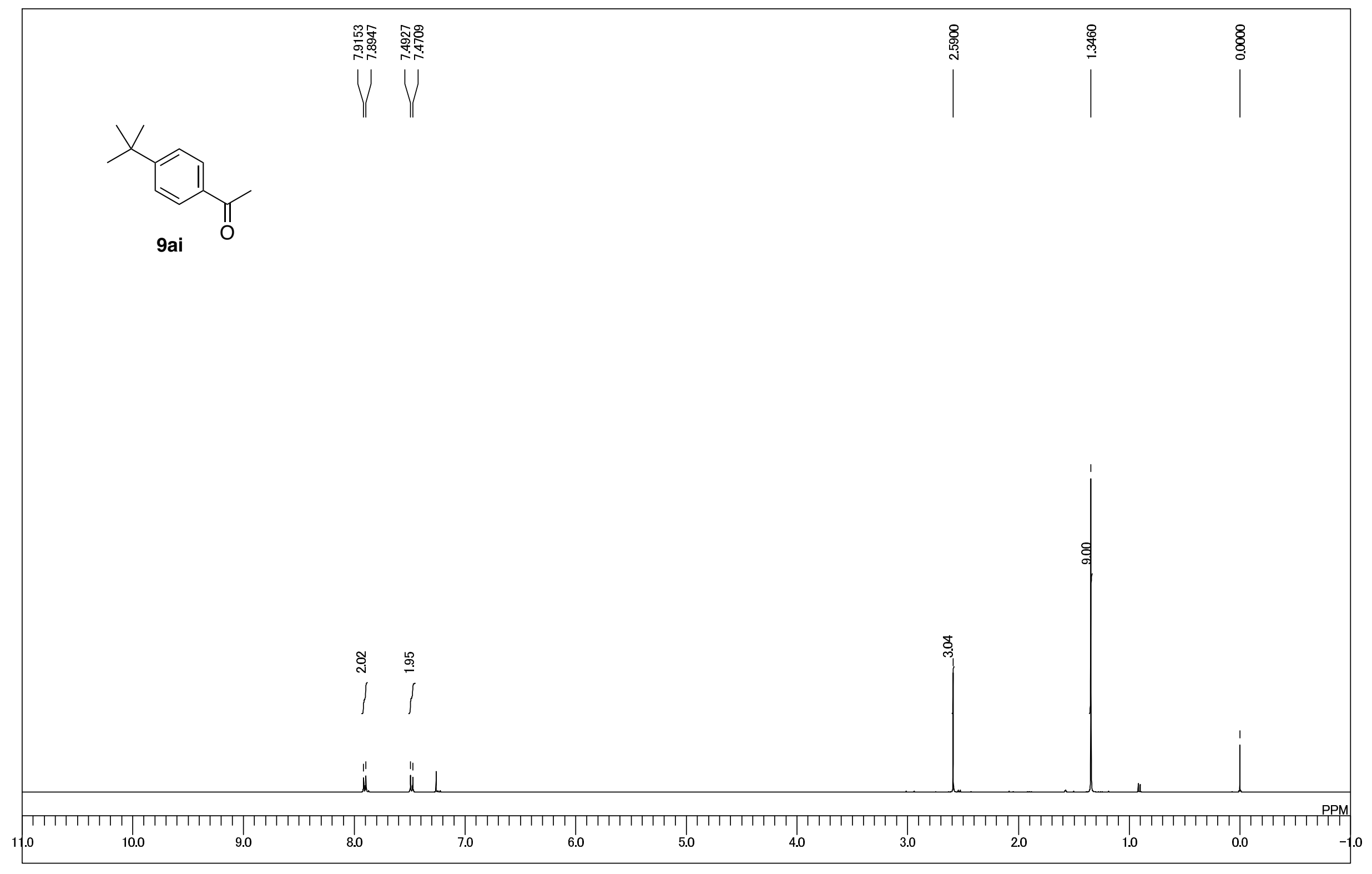




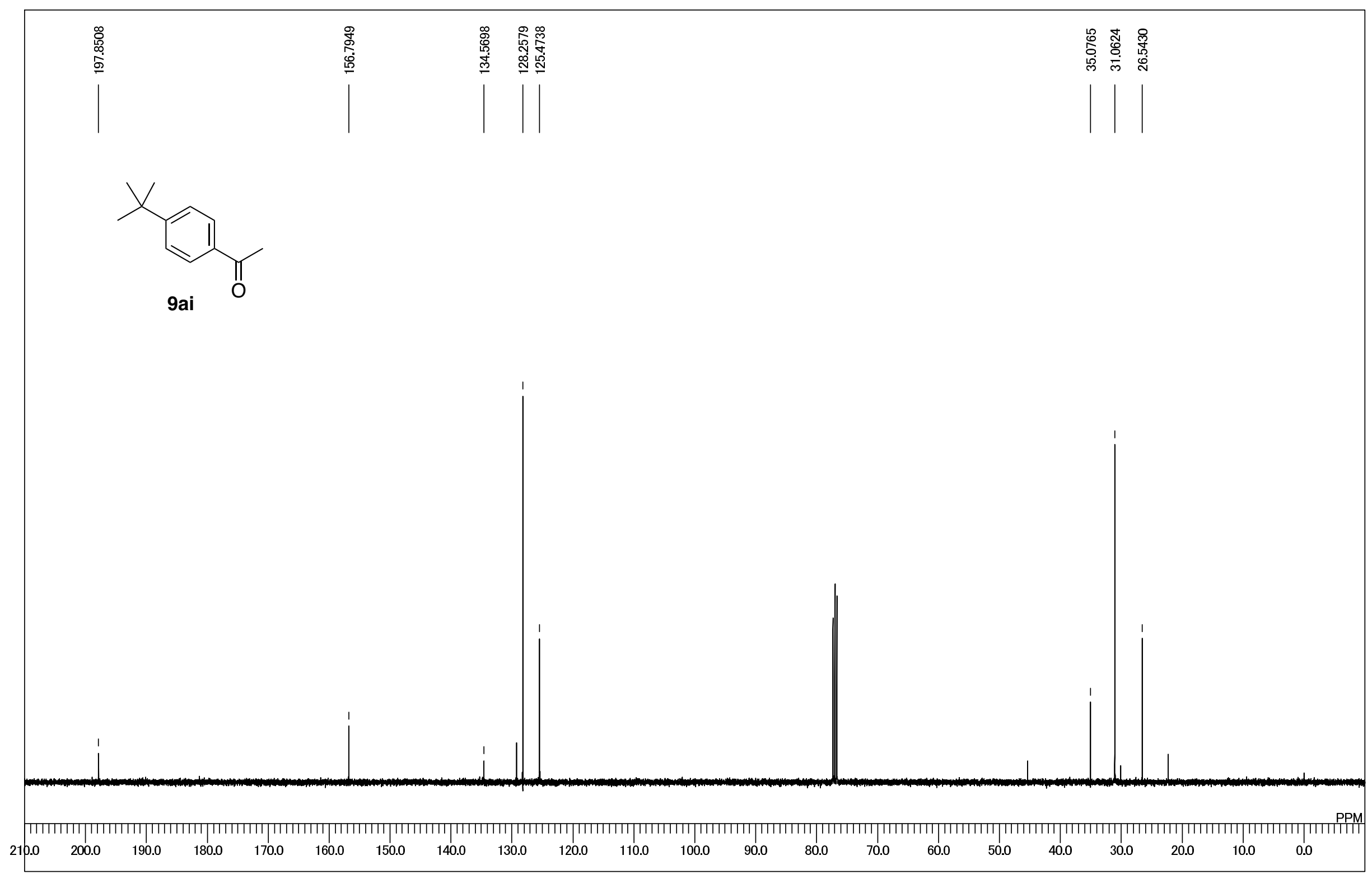




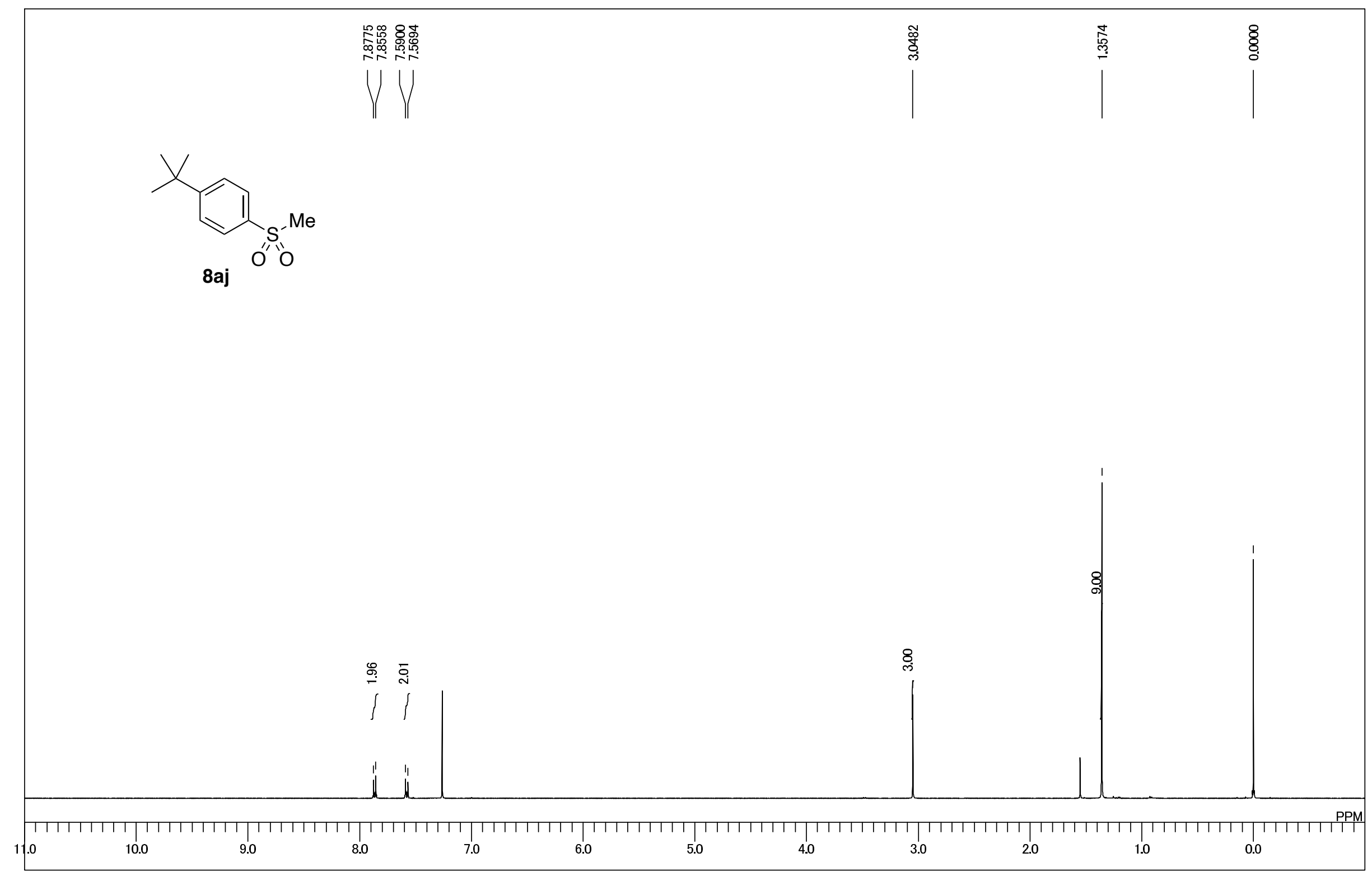




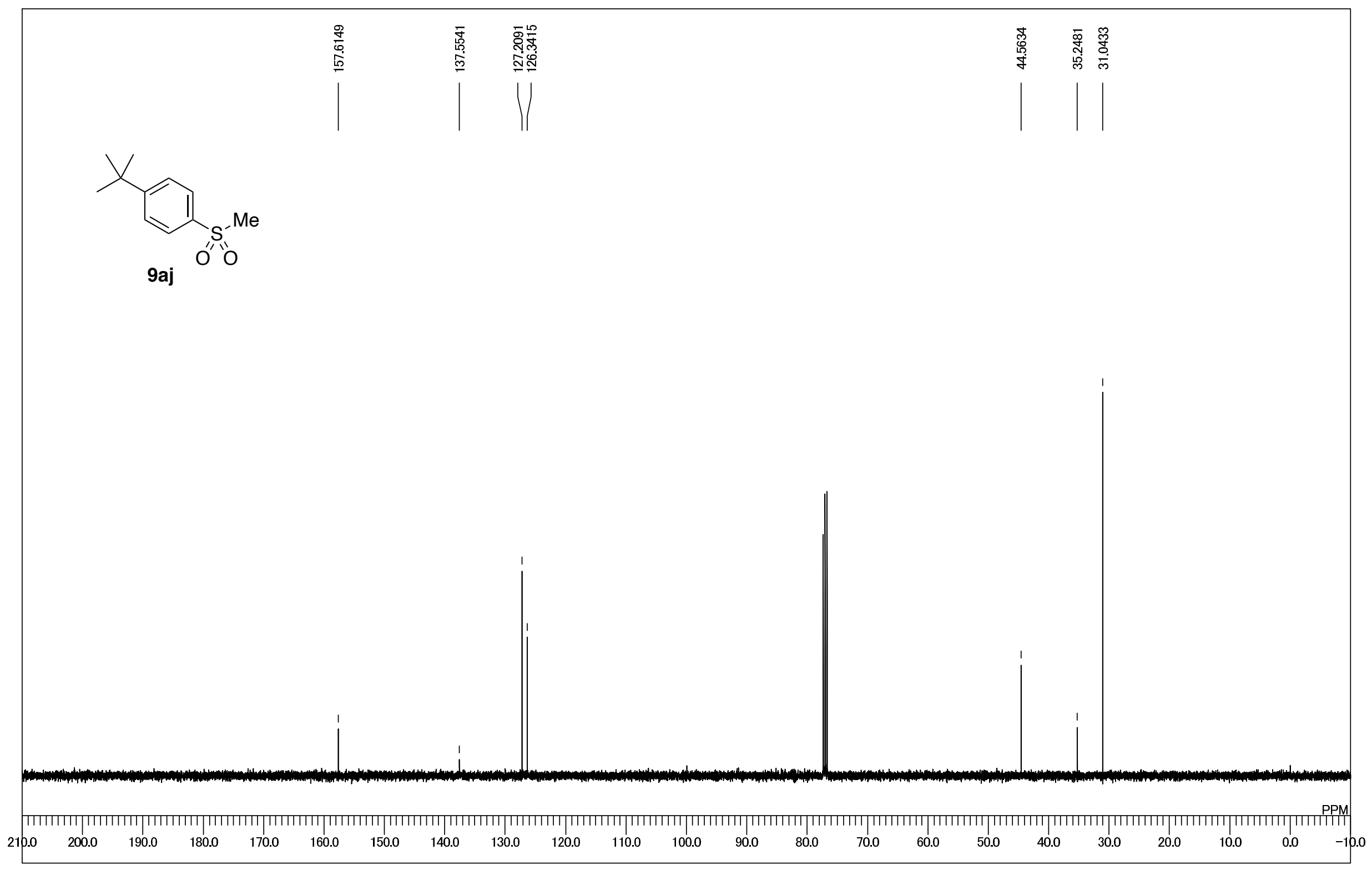




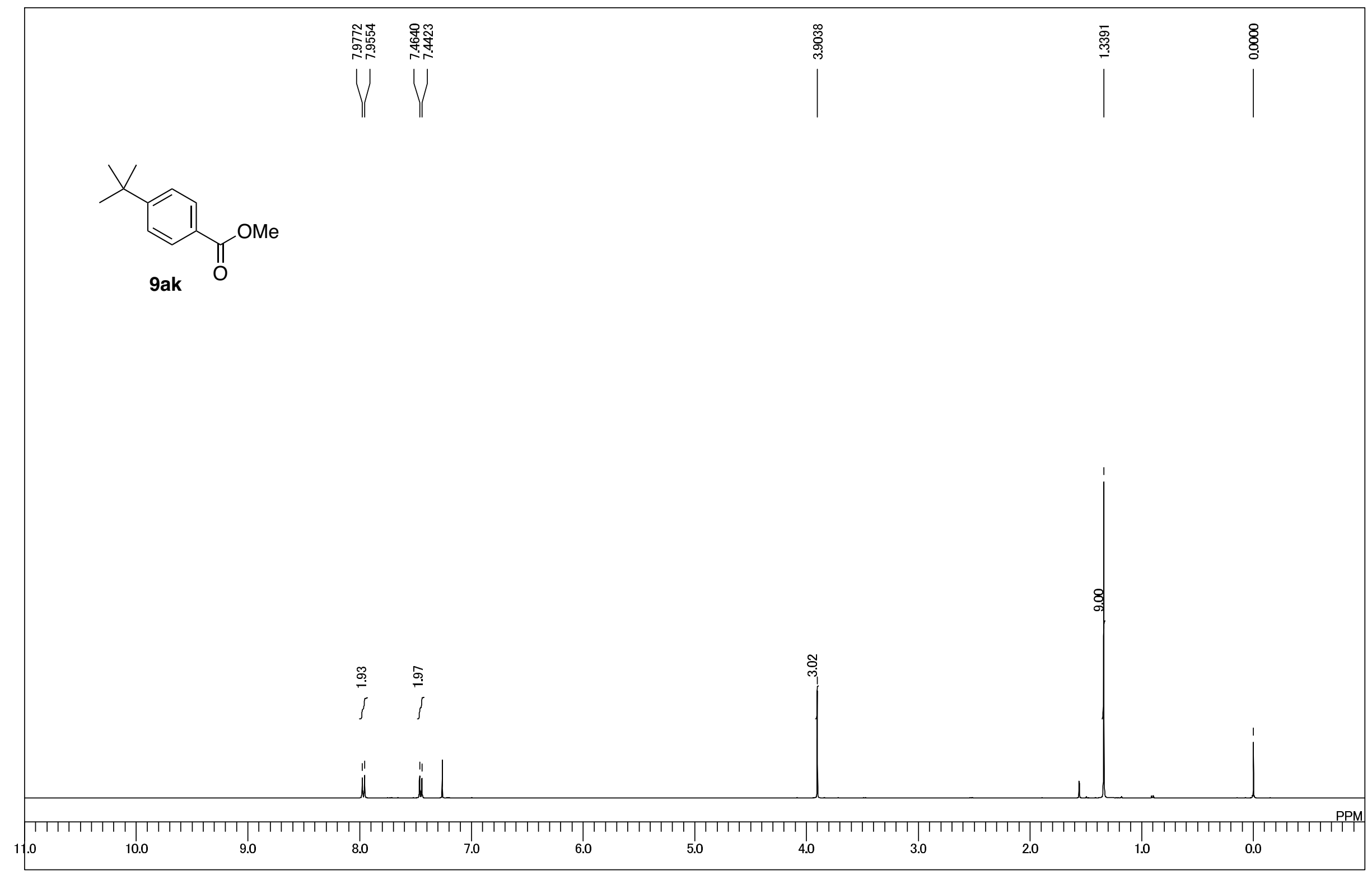




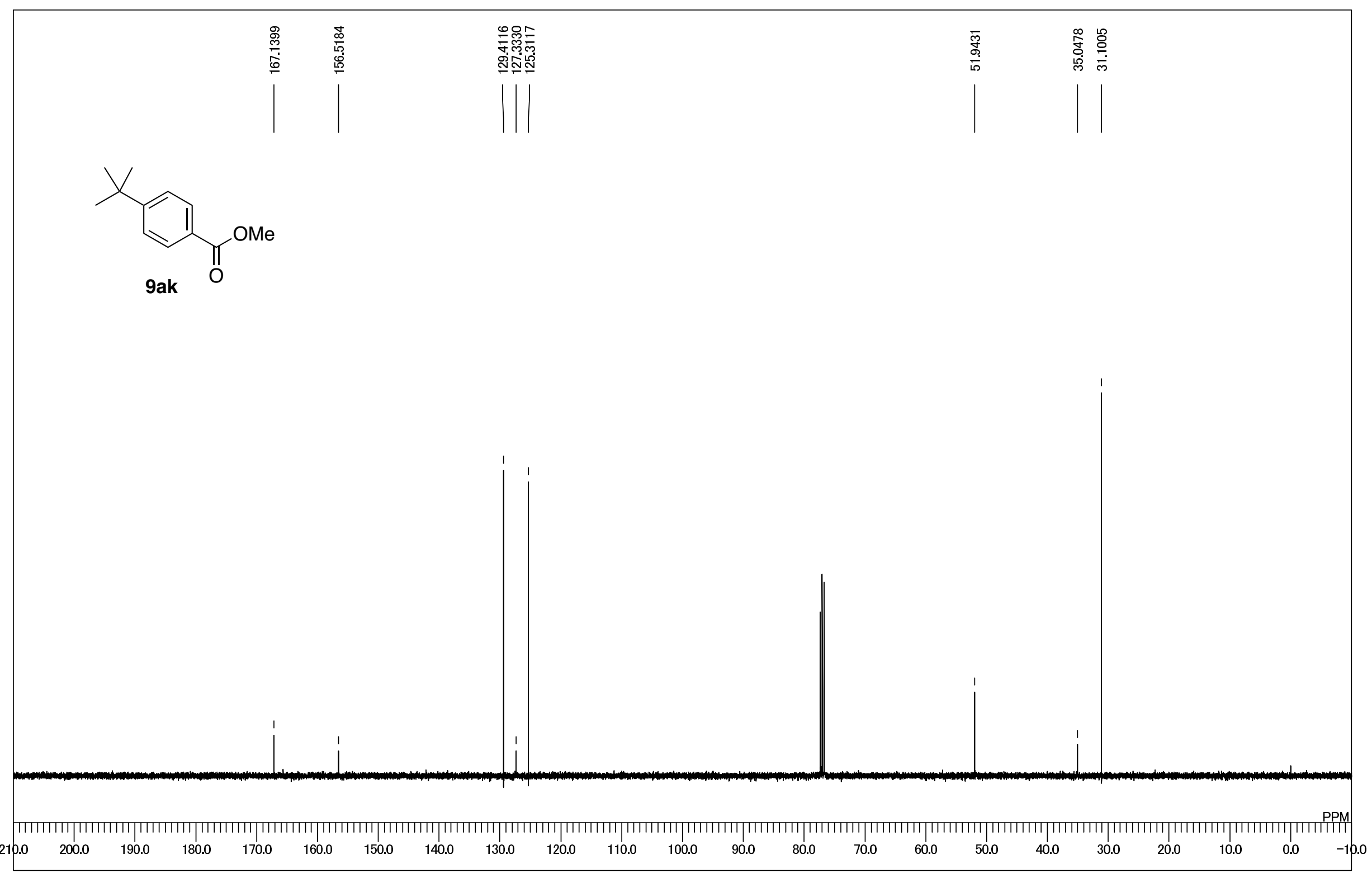




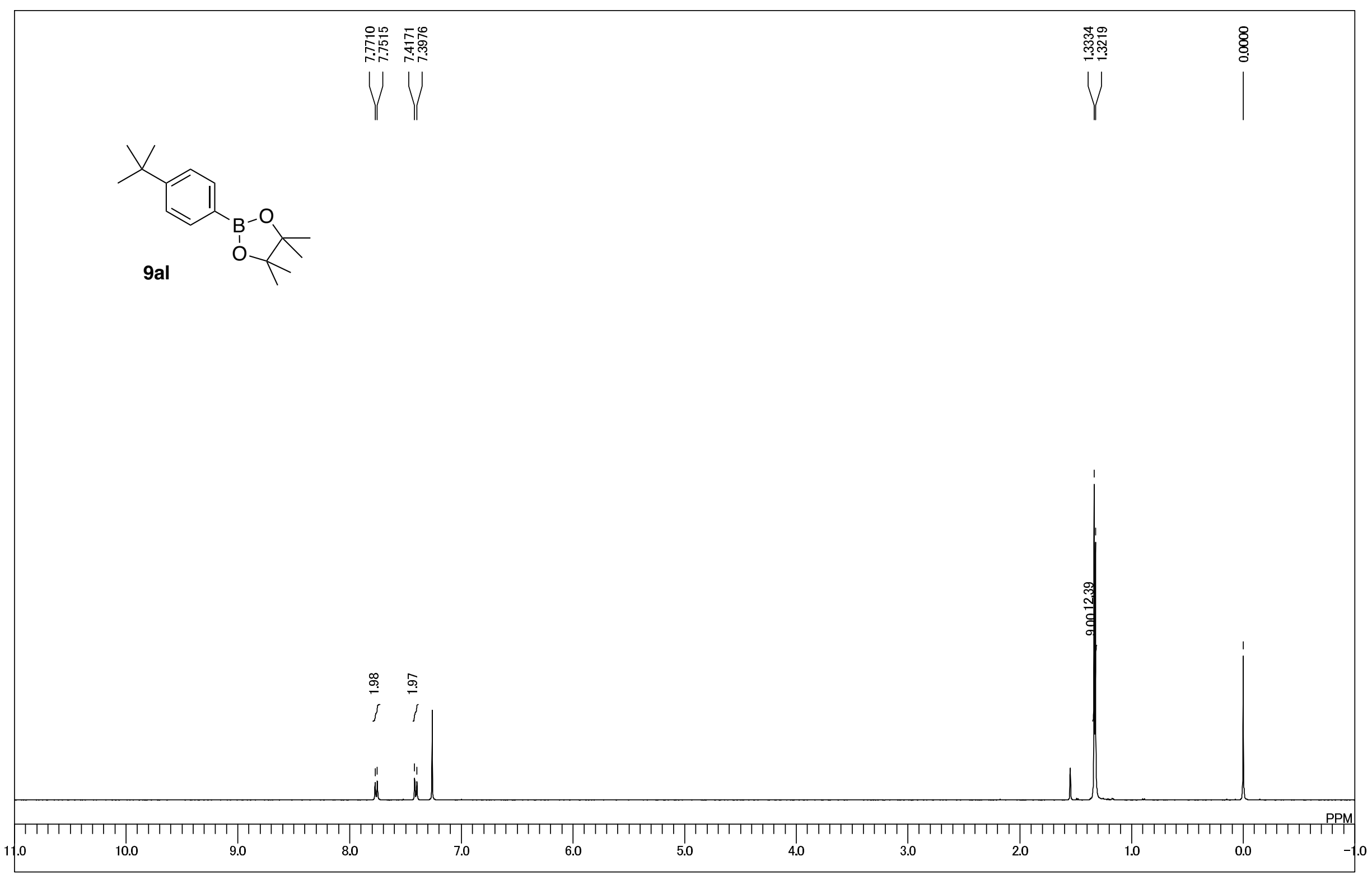

S63 


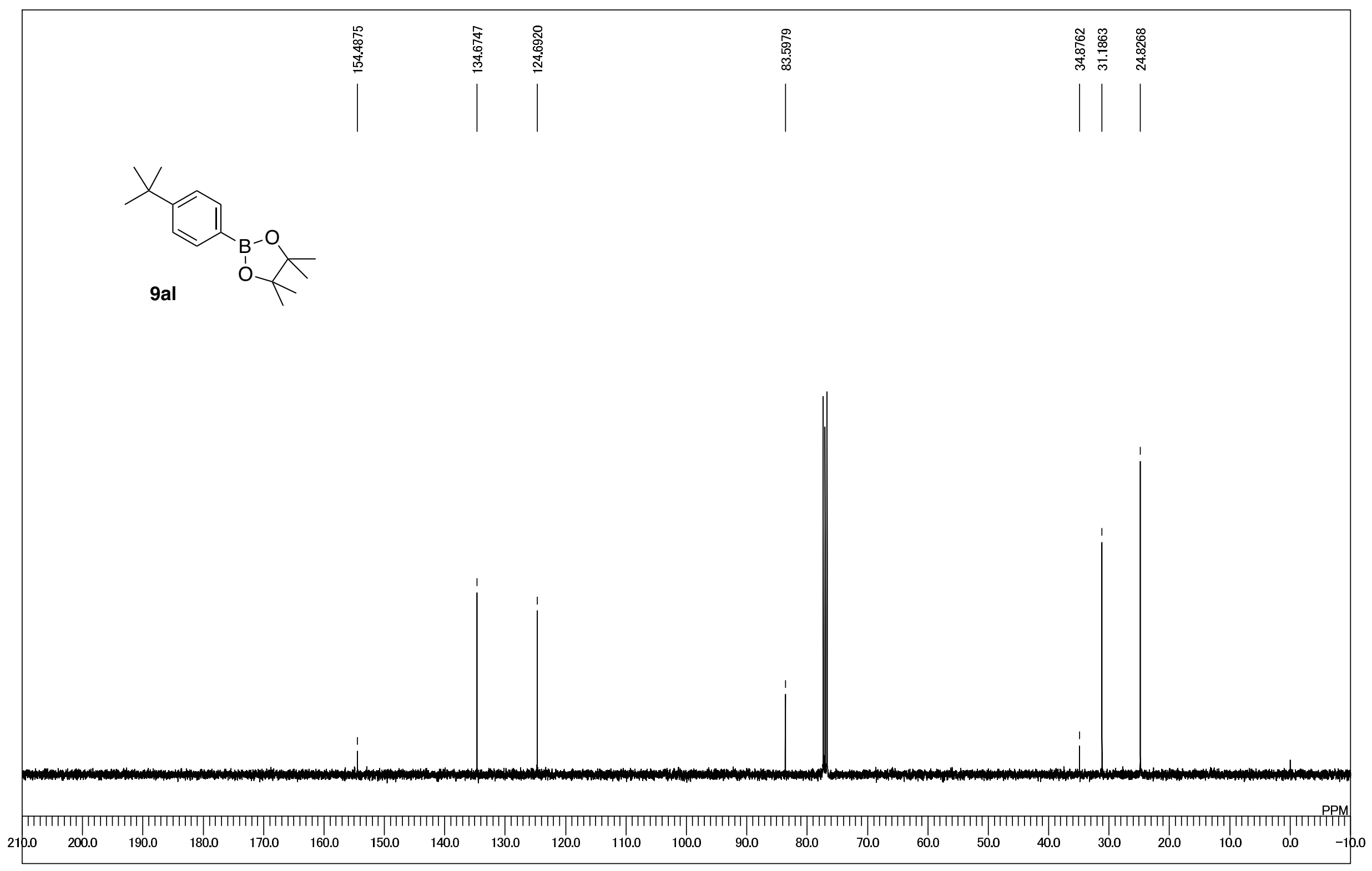




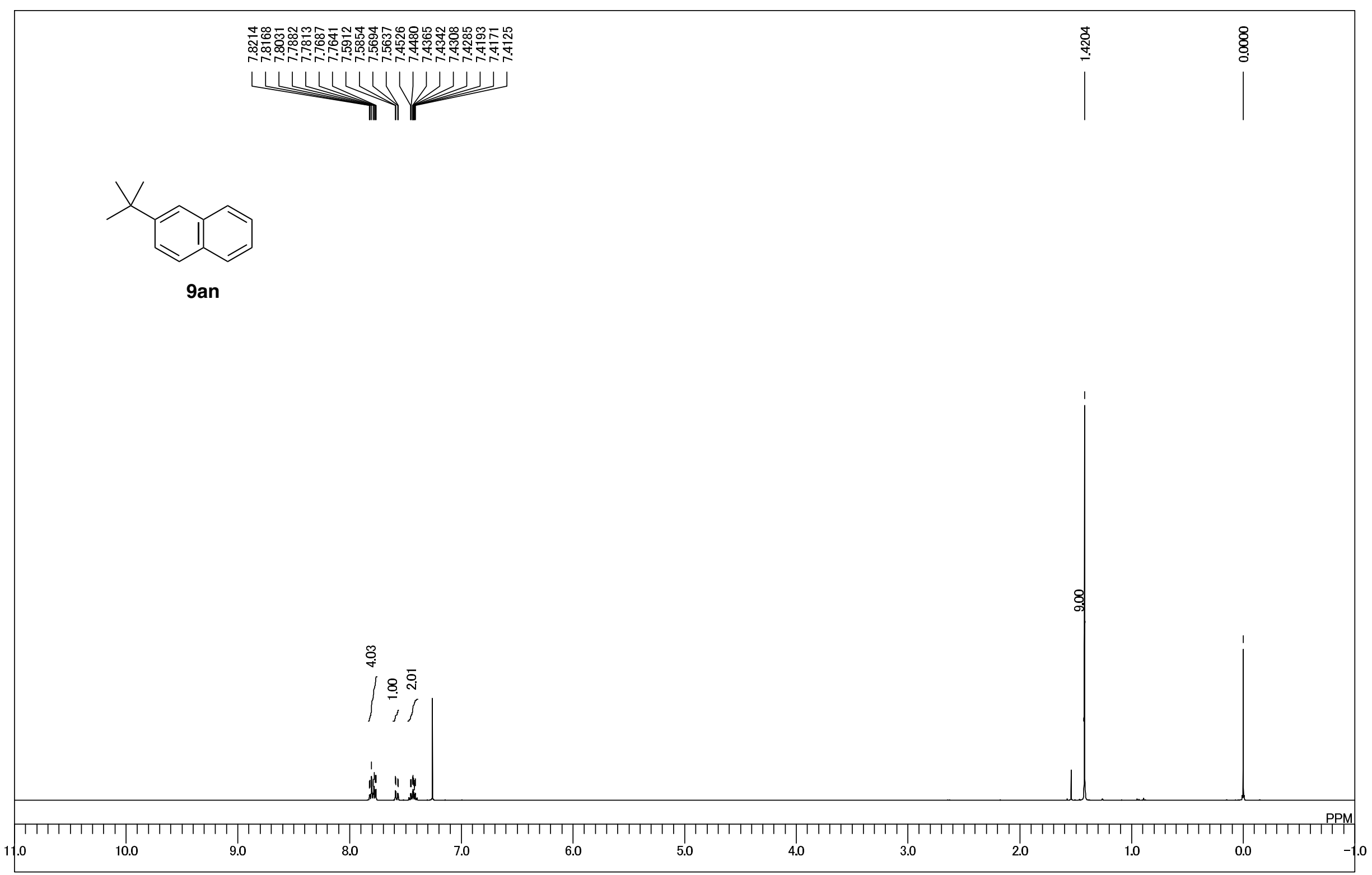

S65 


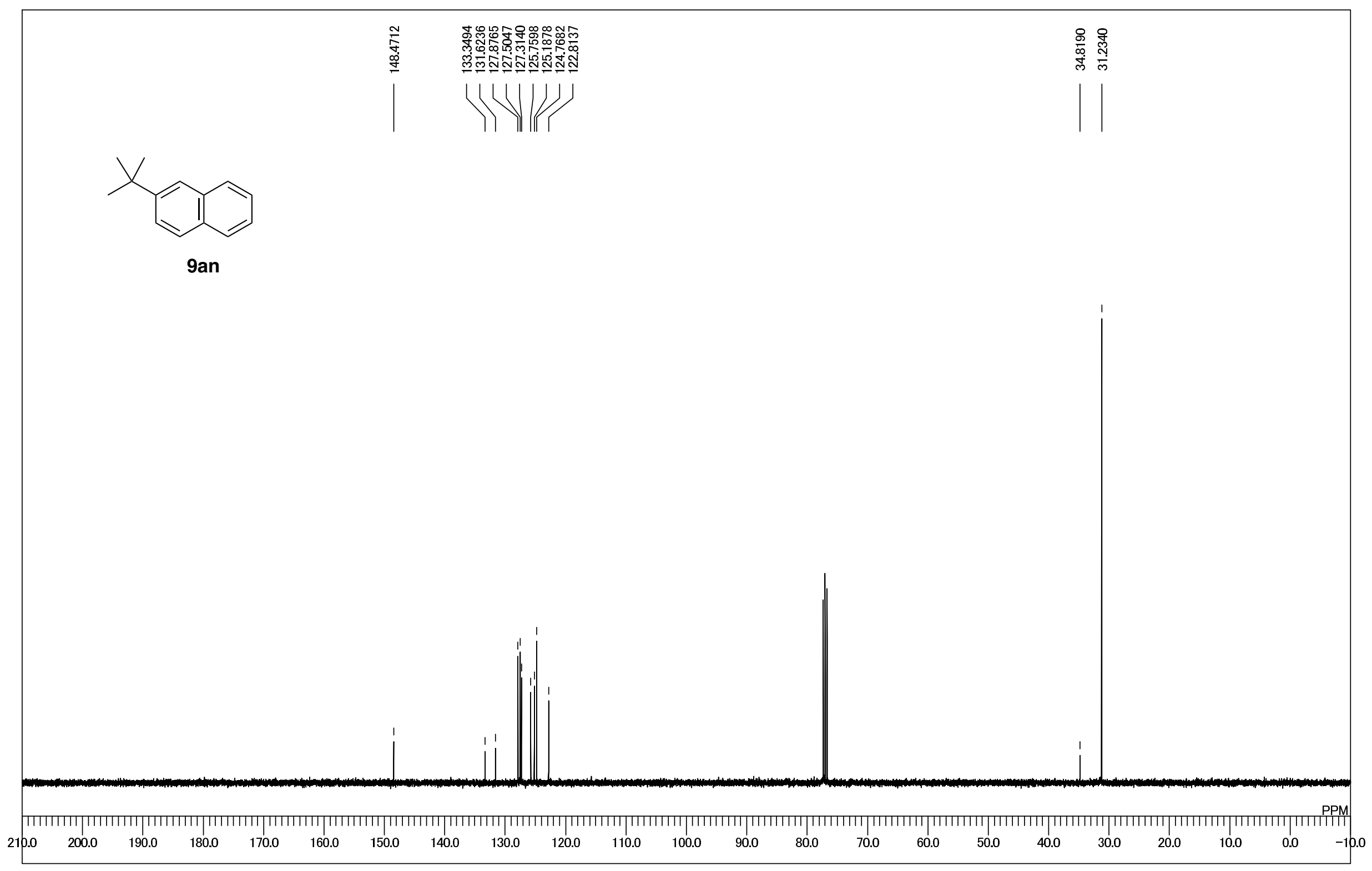




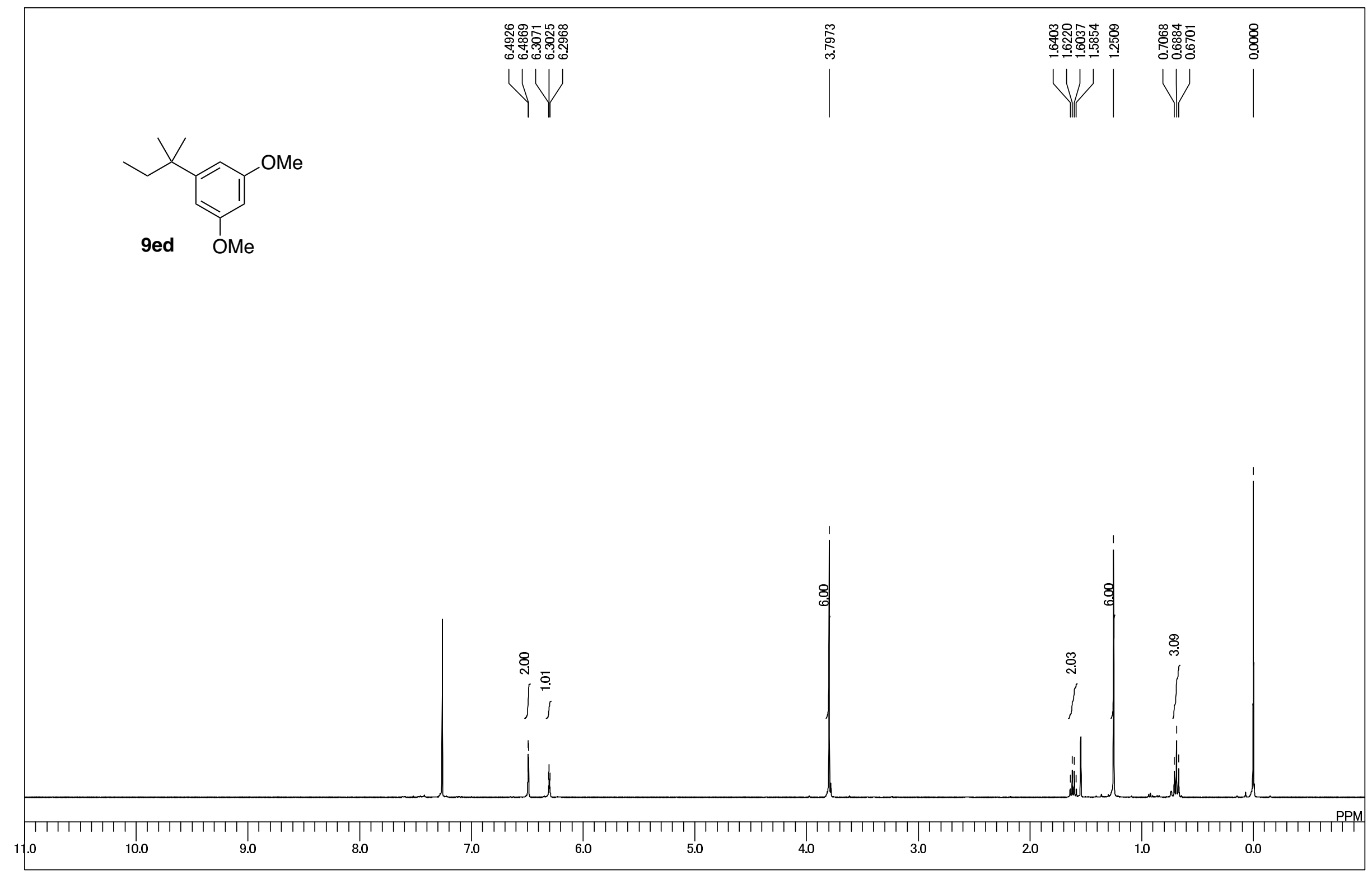




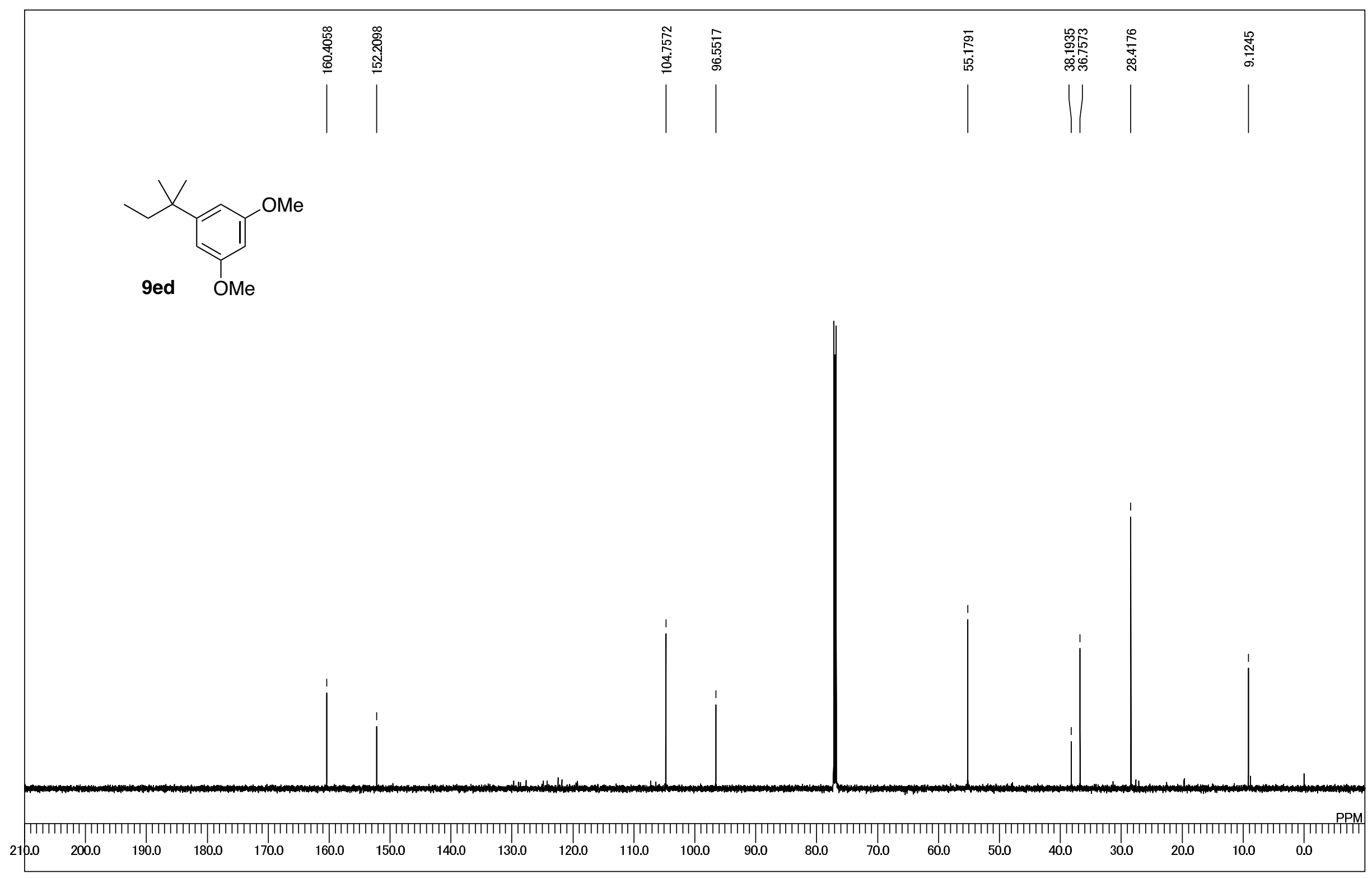




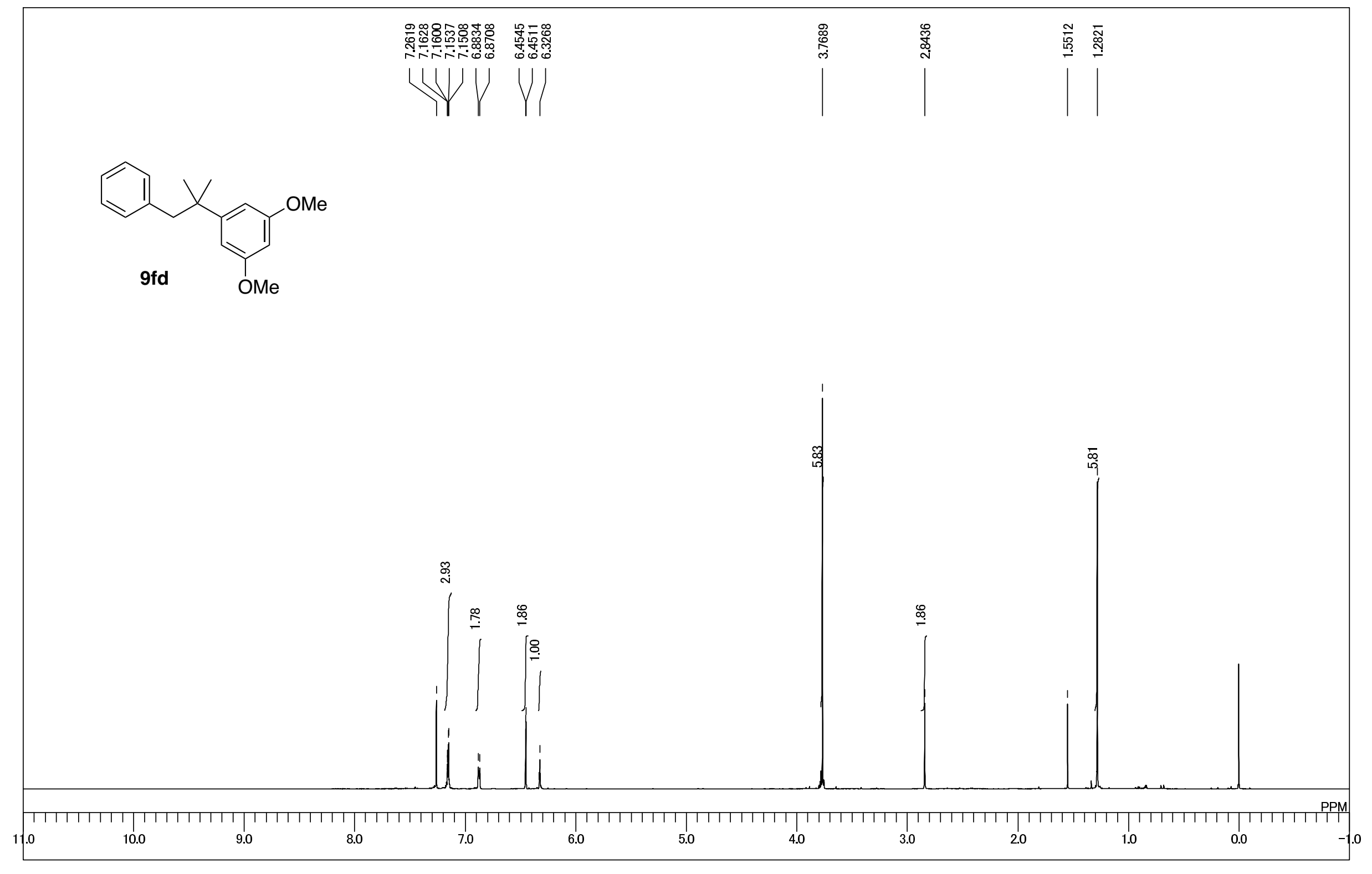




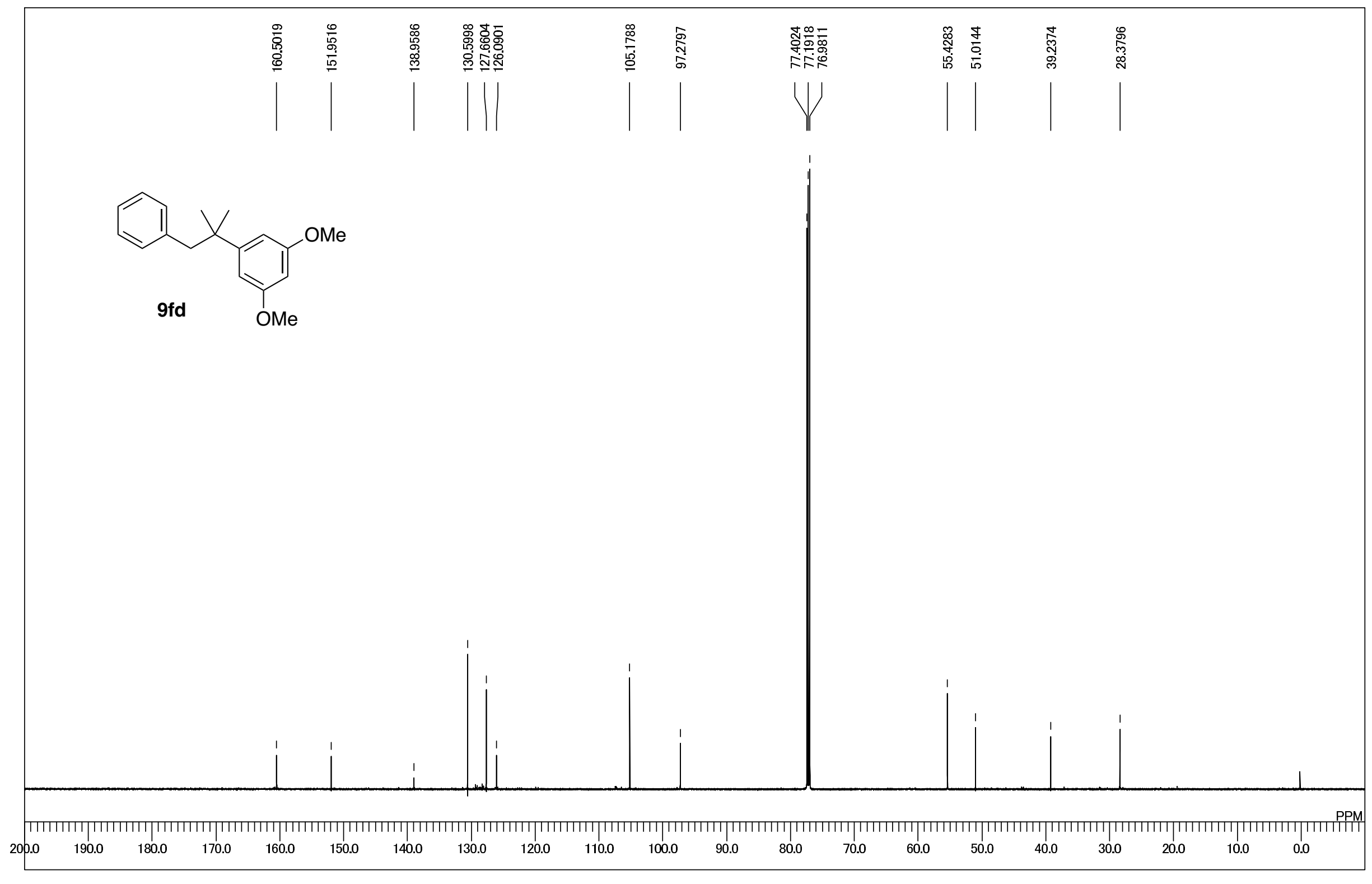




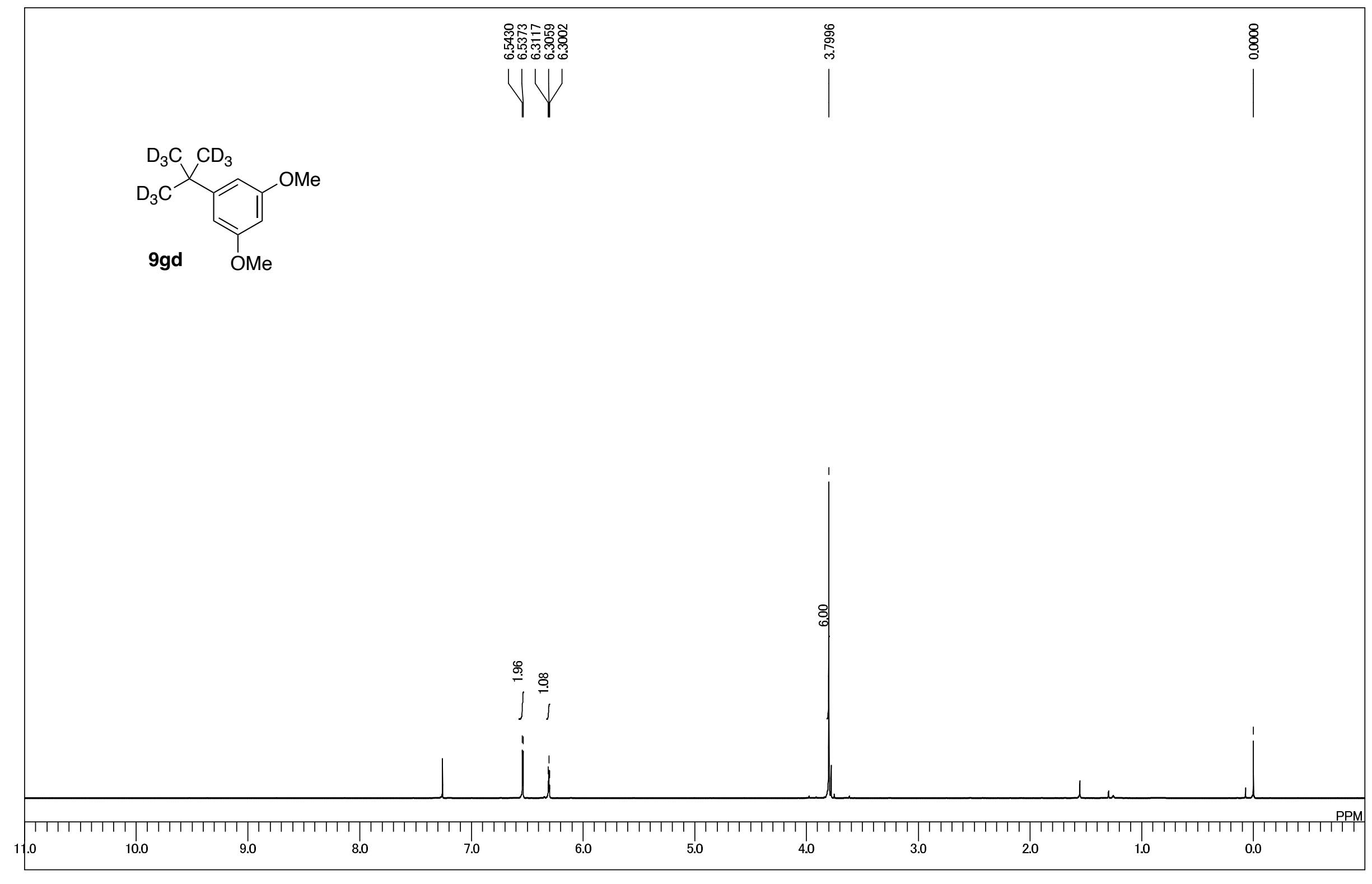




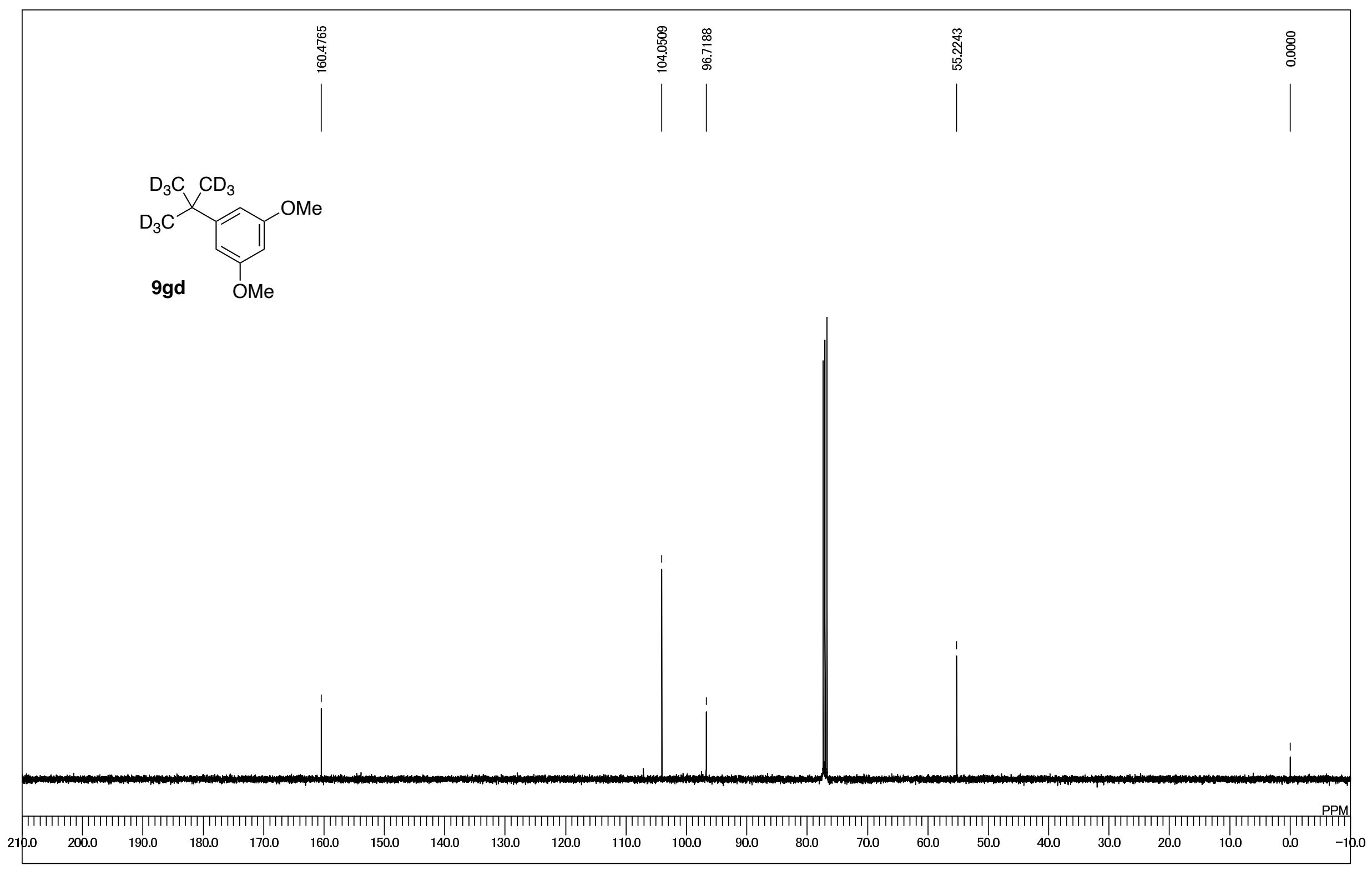




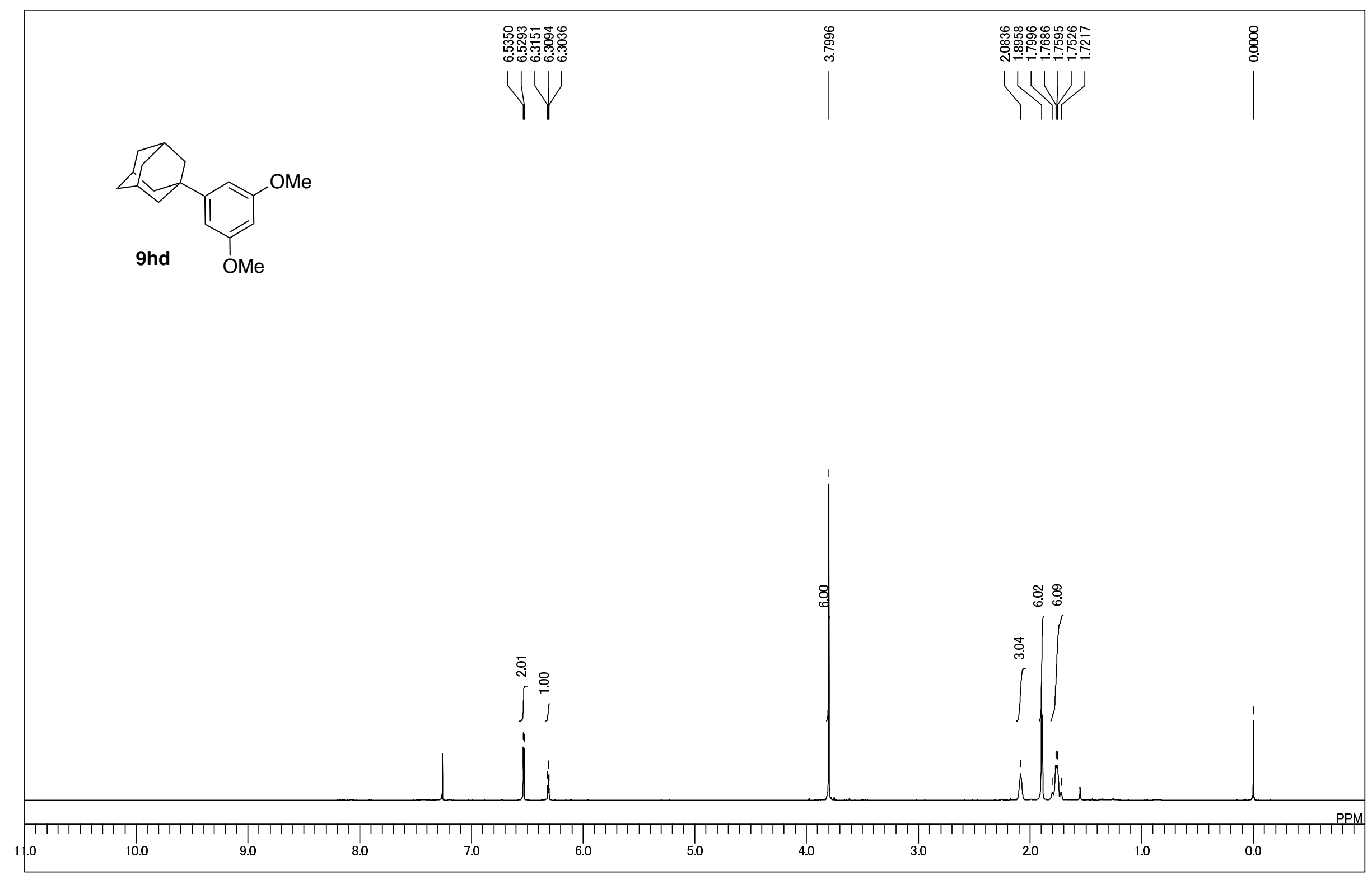




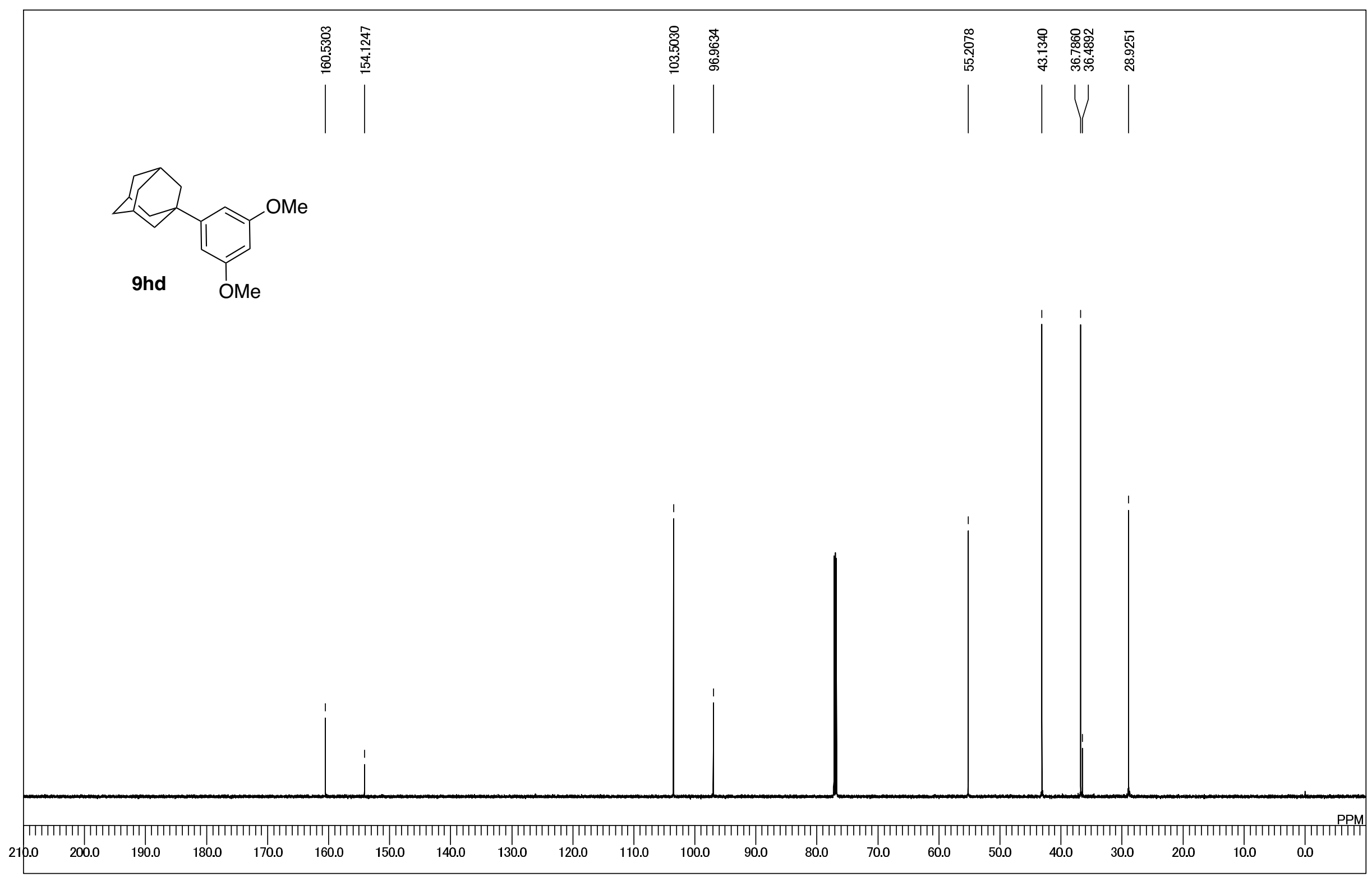




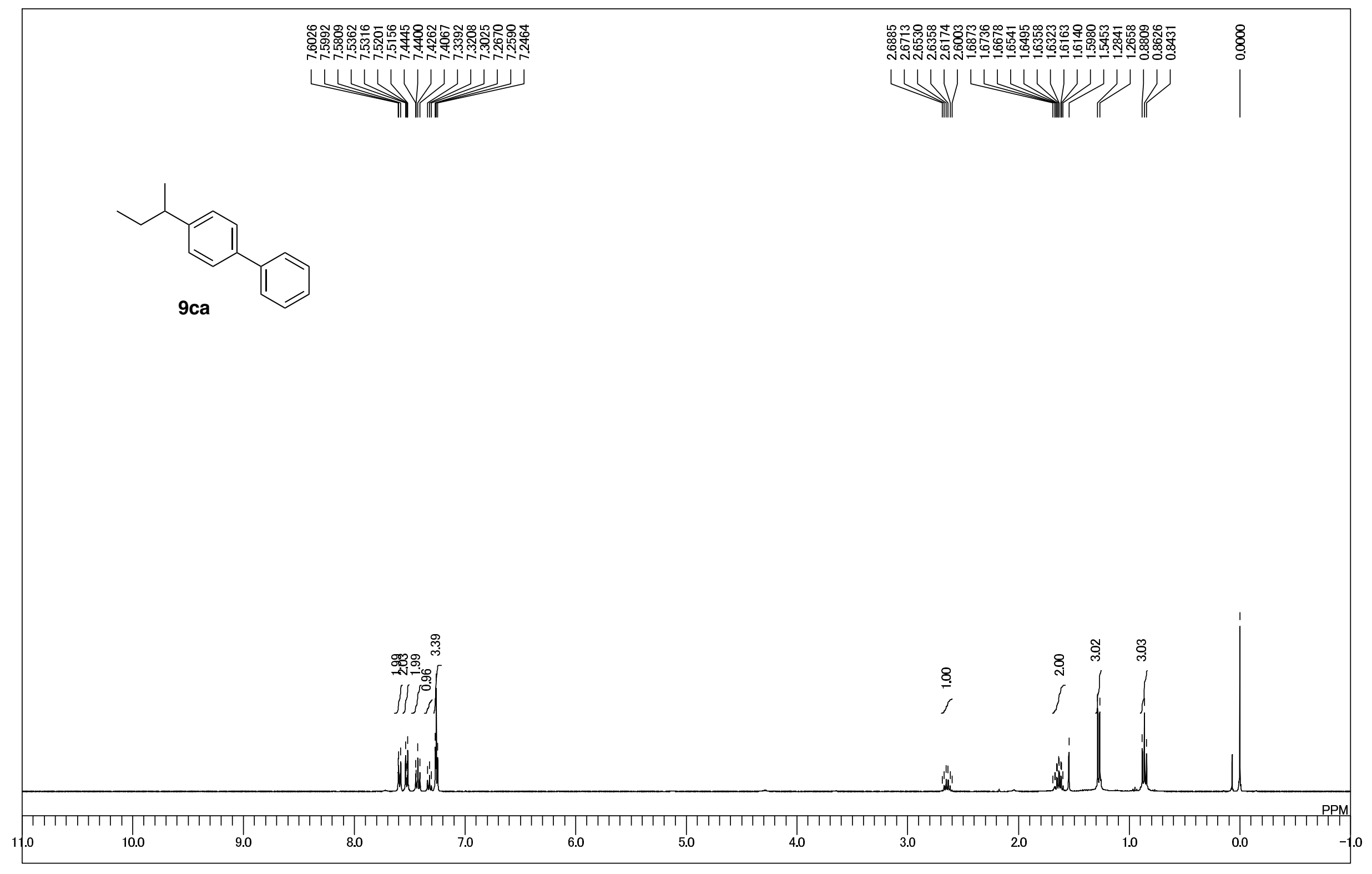




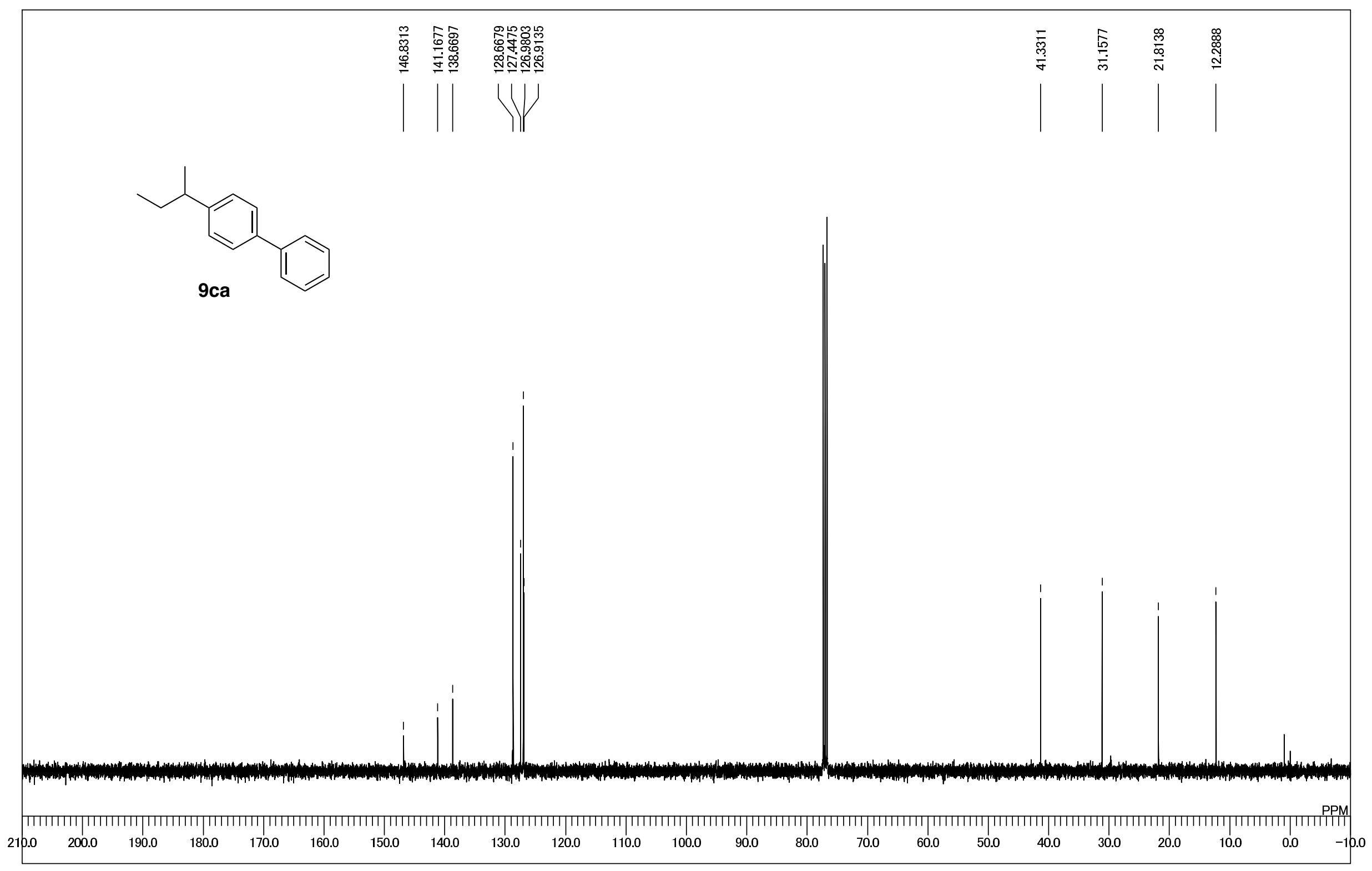




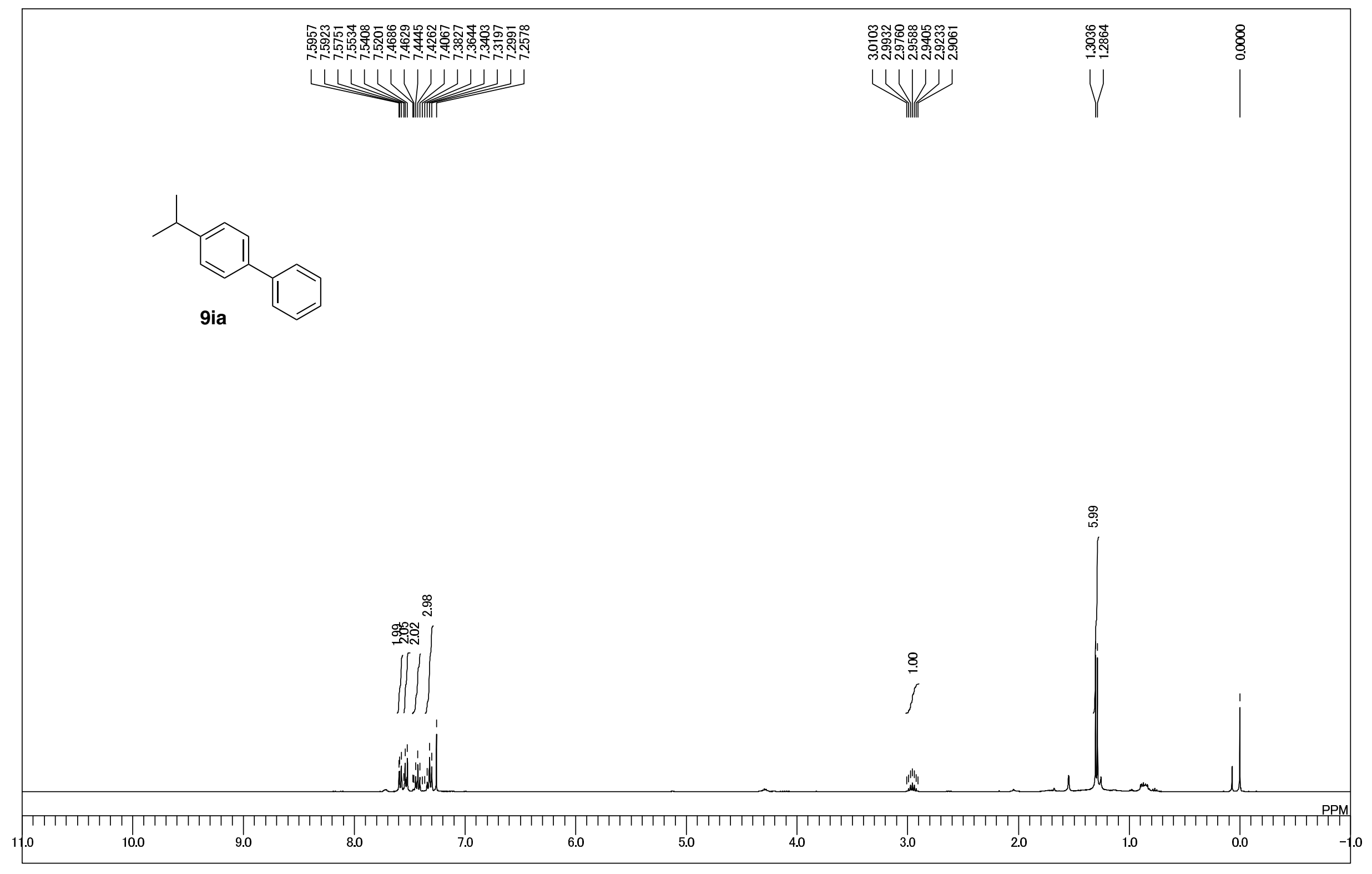




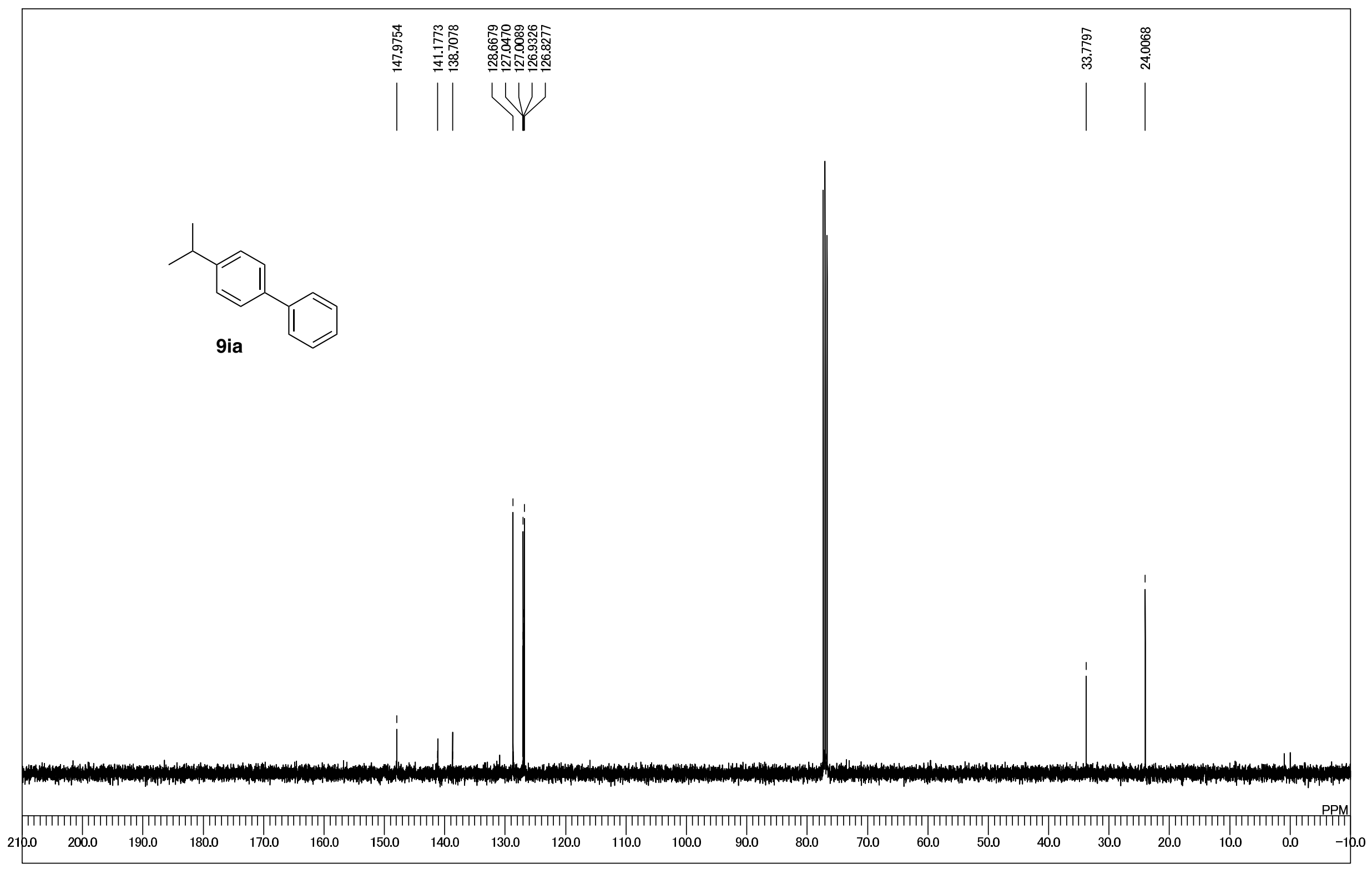




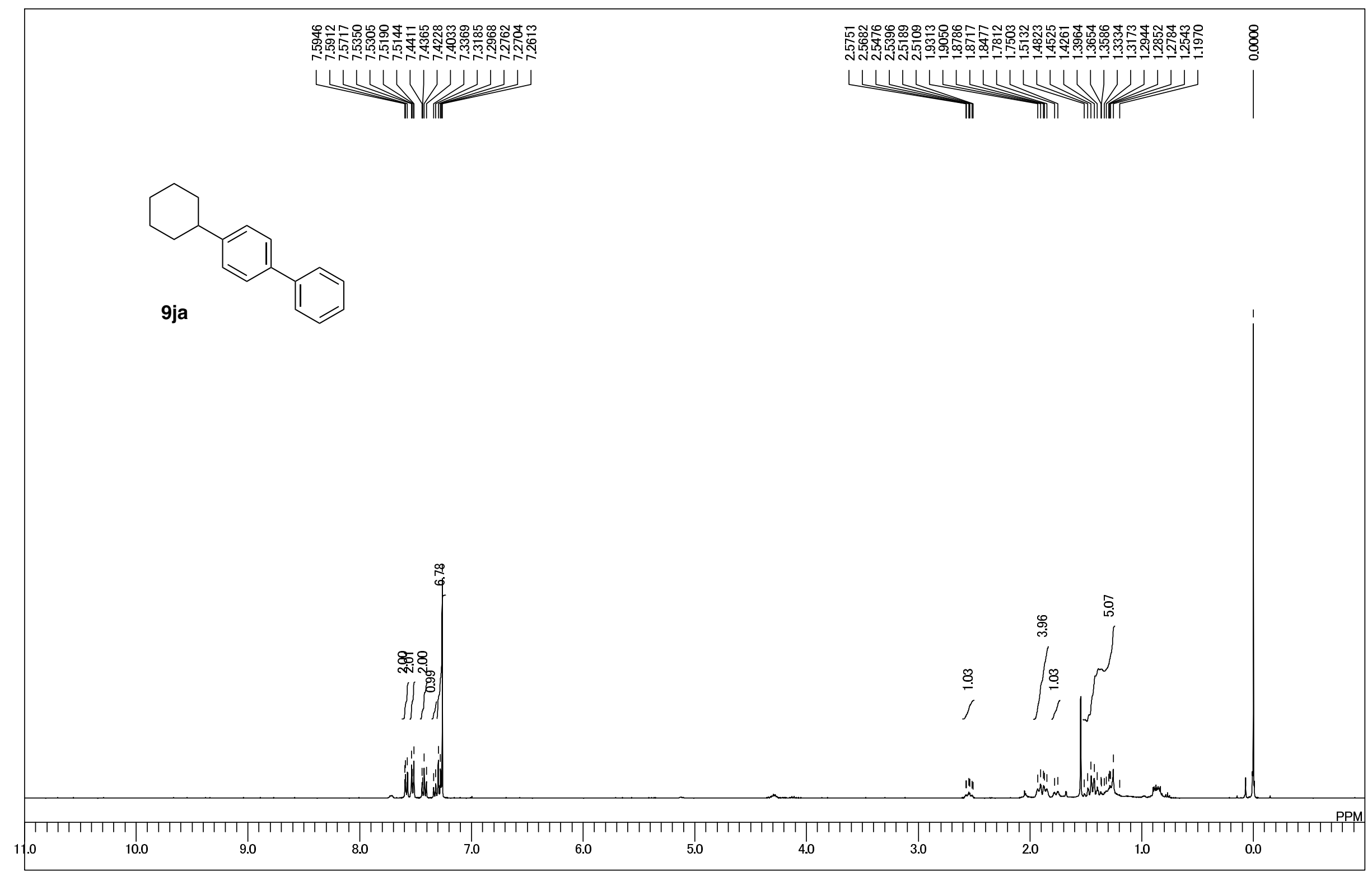




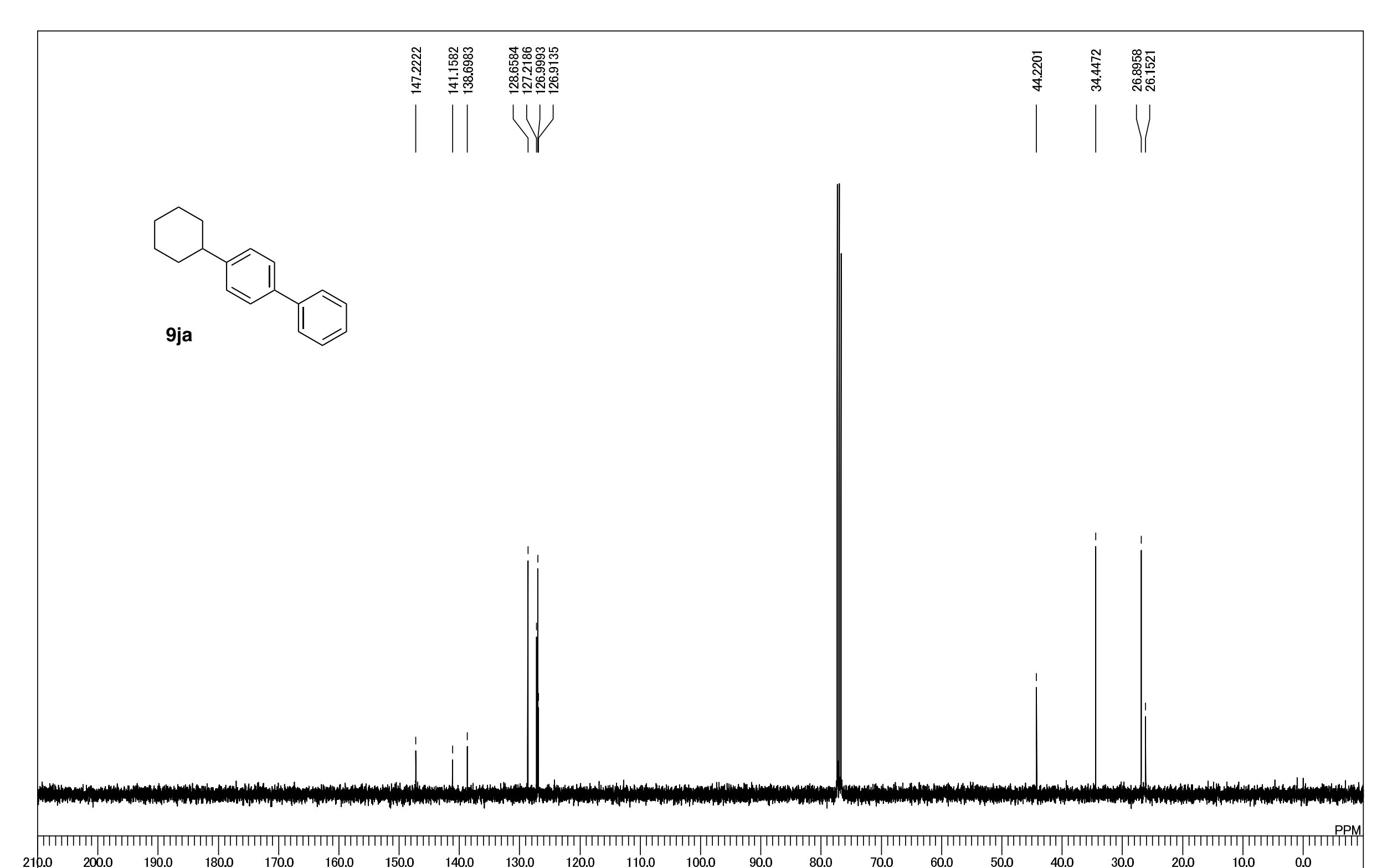




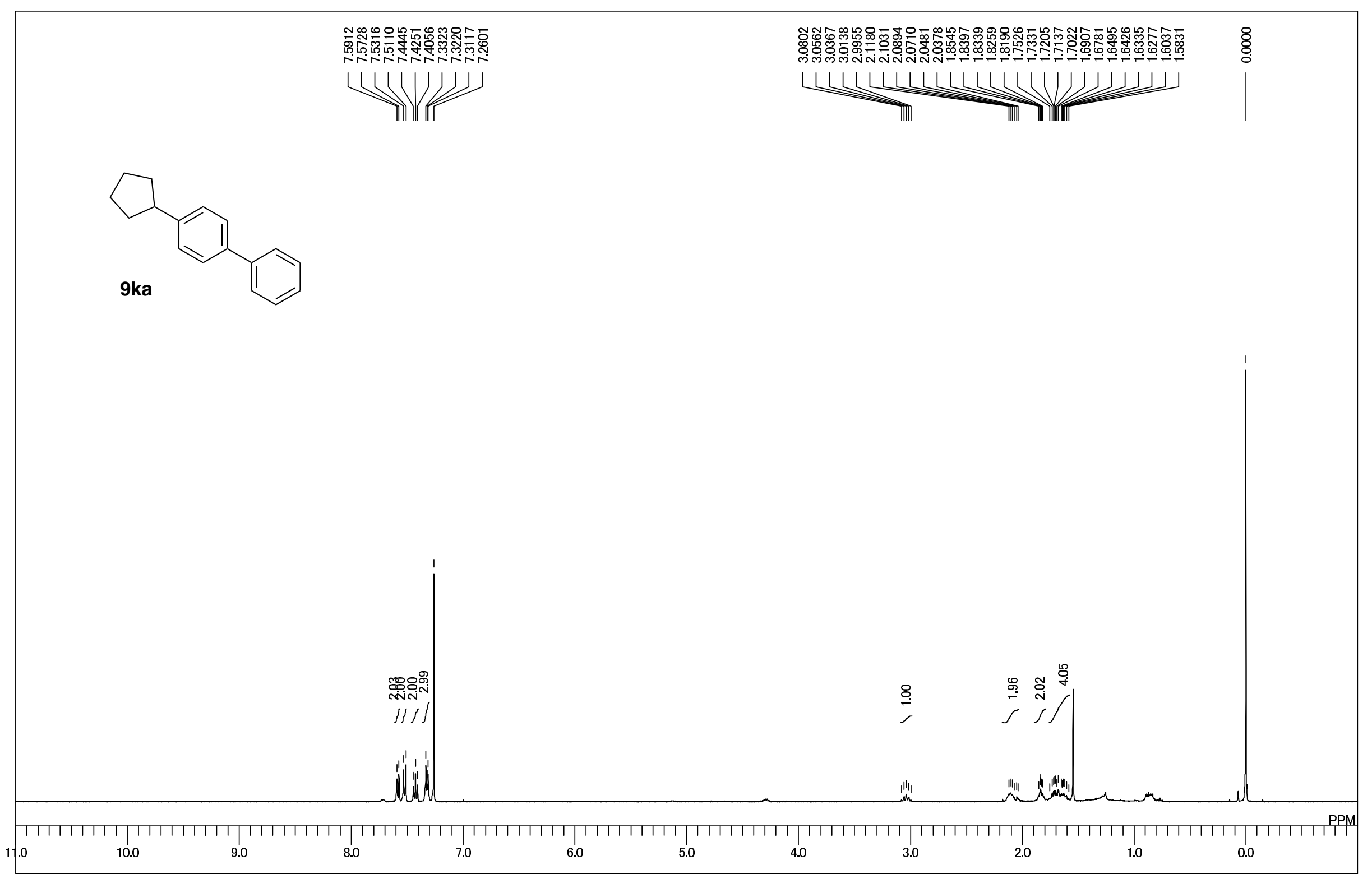

S81 


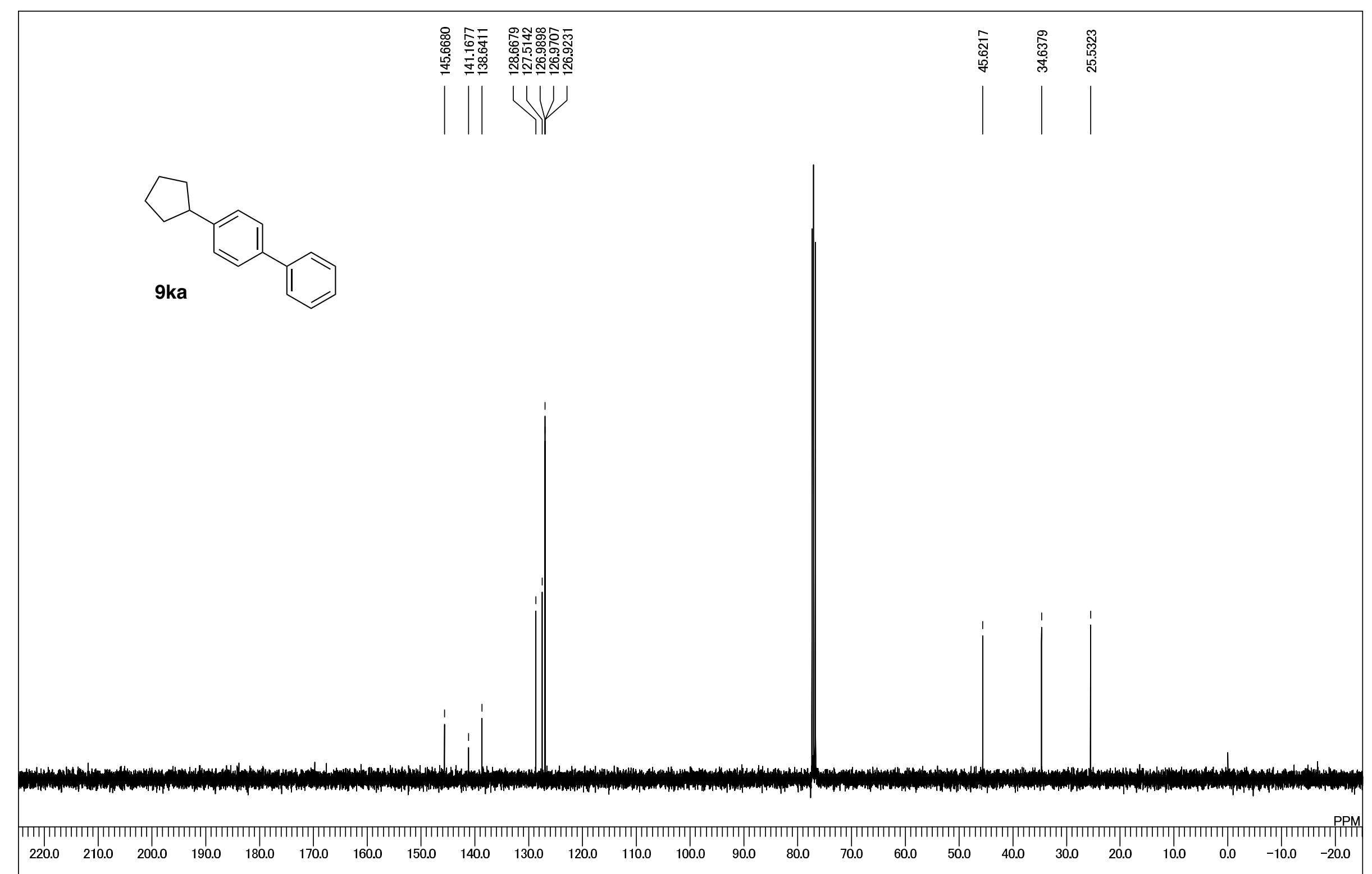

S82 


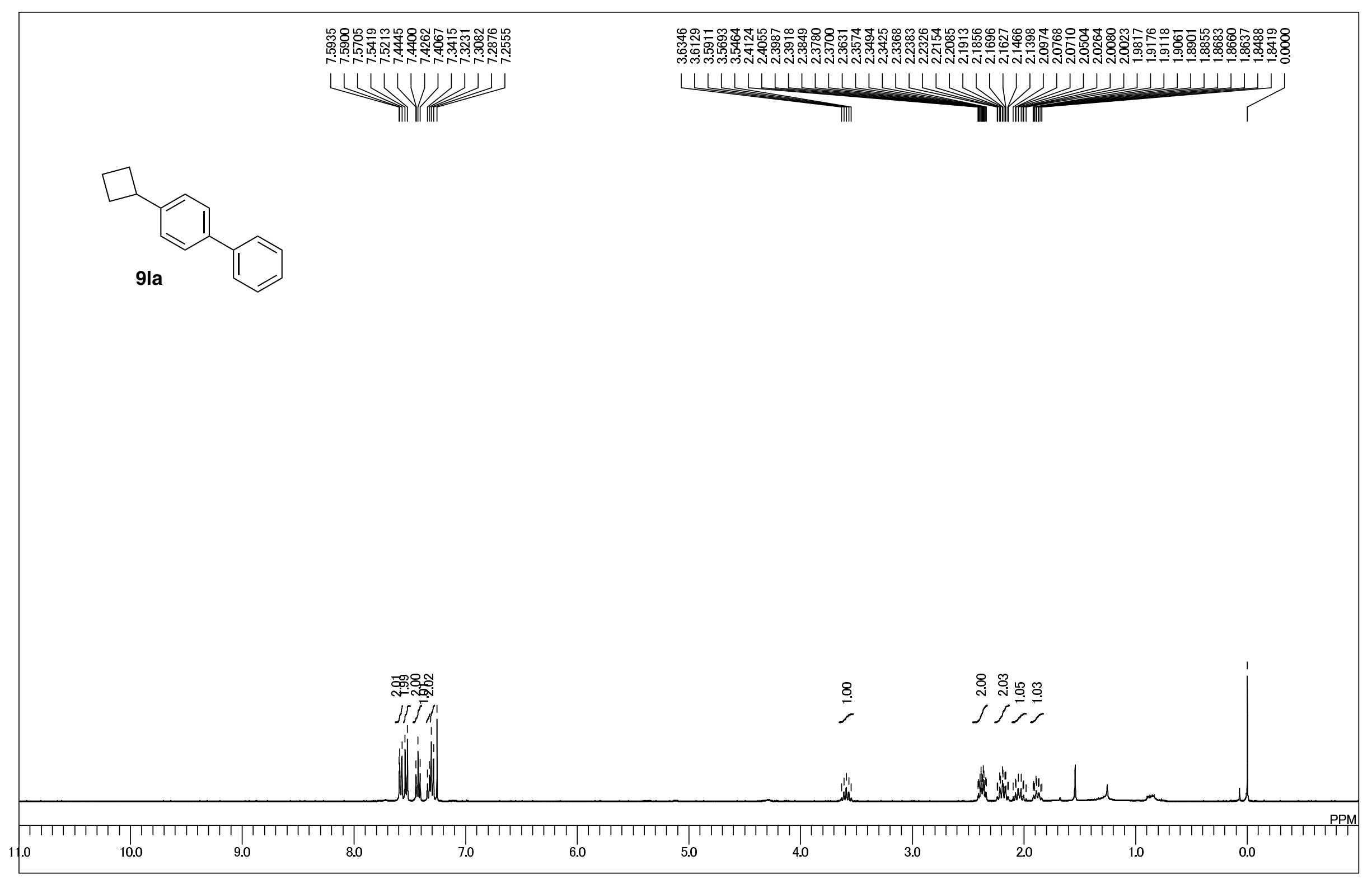

S83 


$$
41
$$




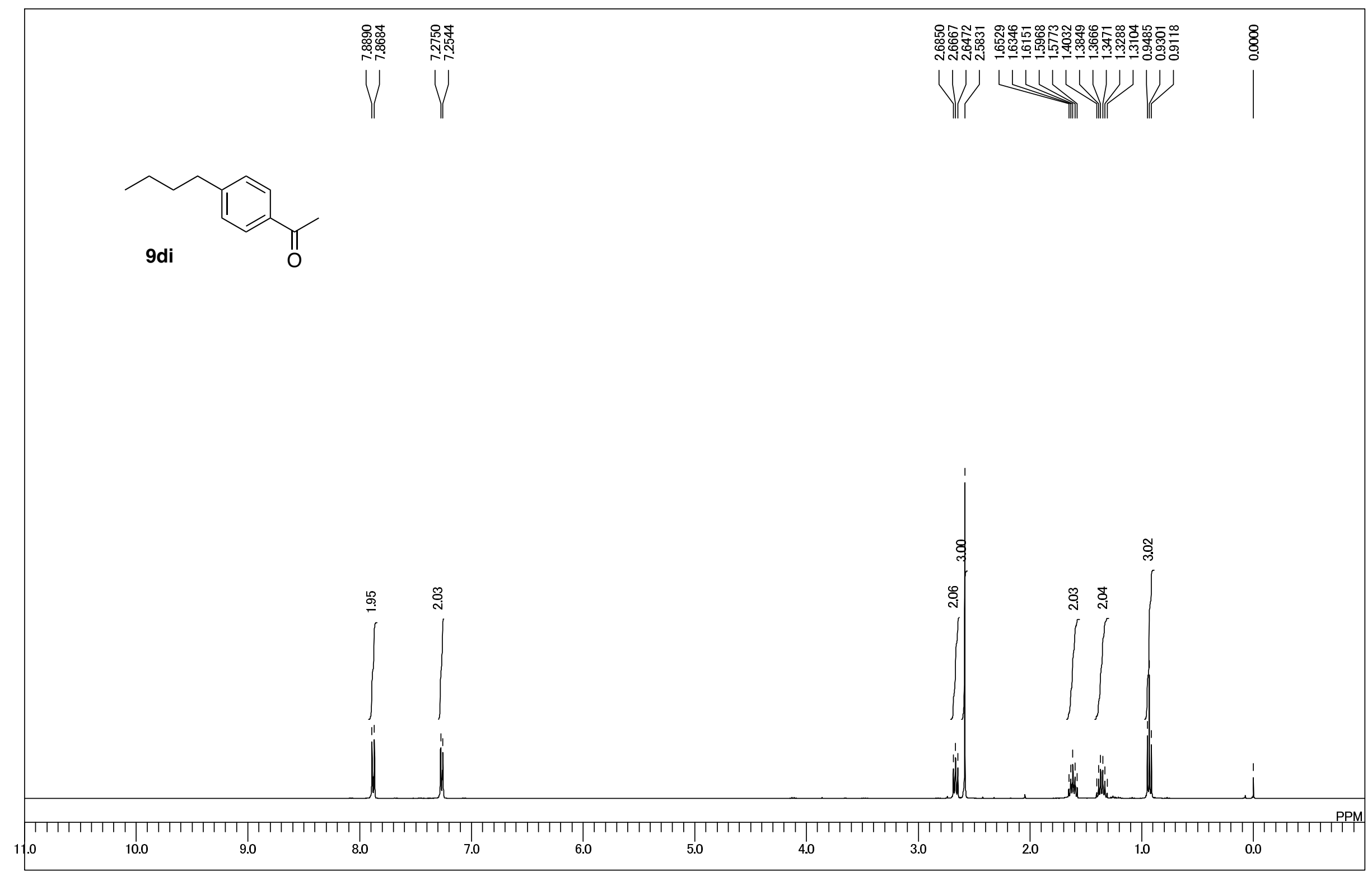




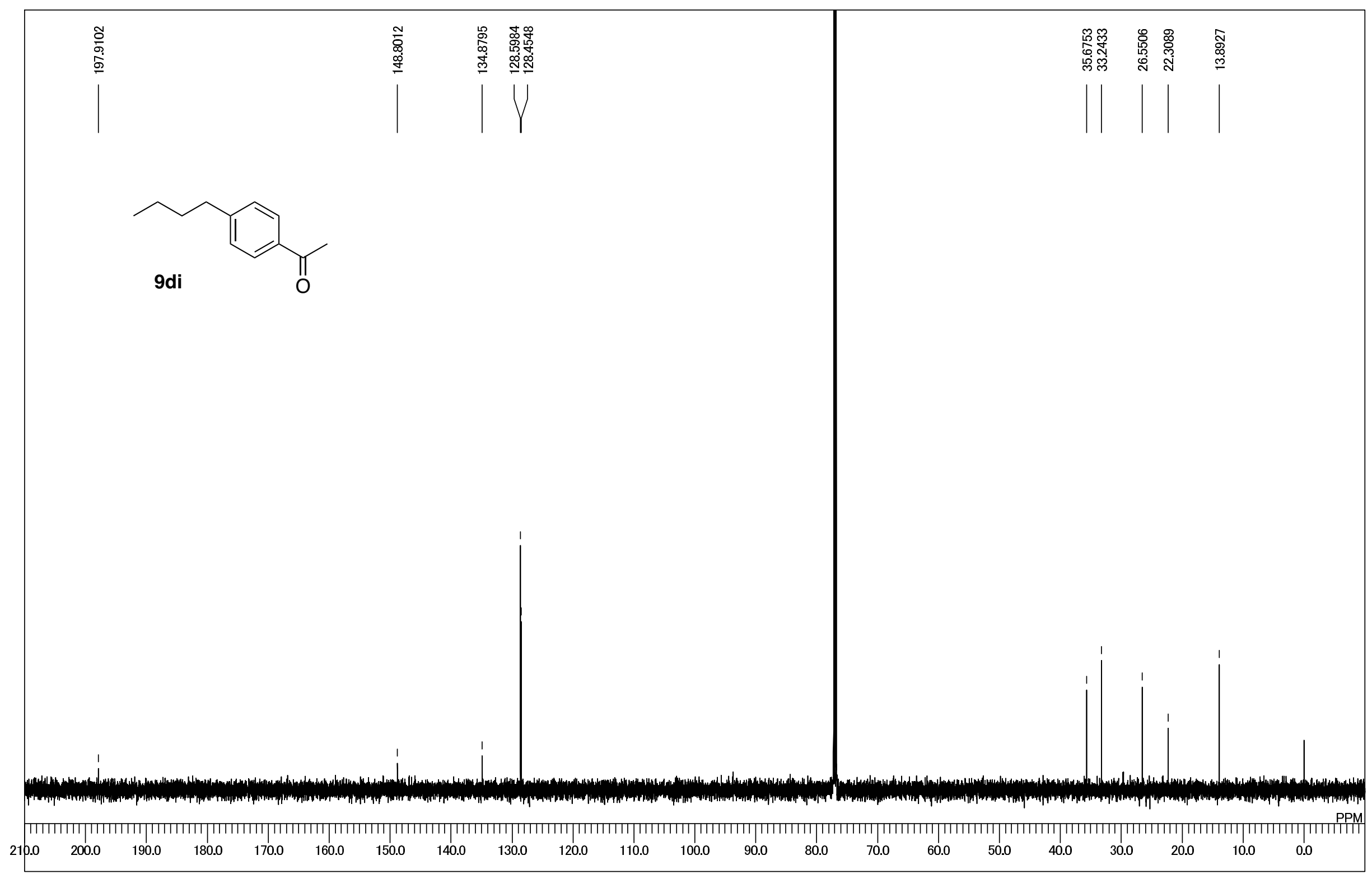




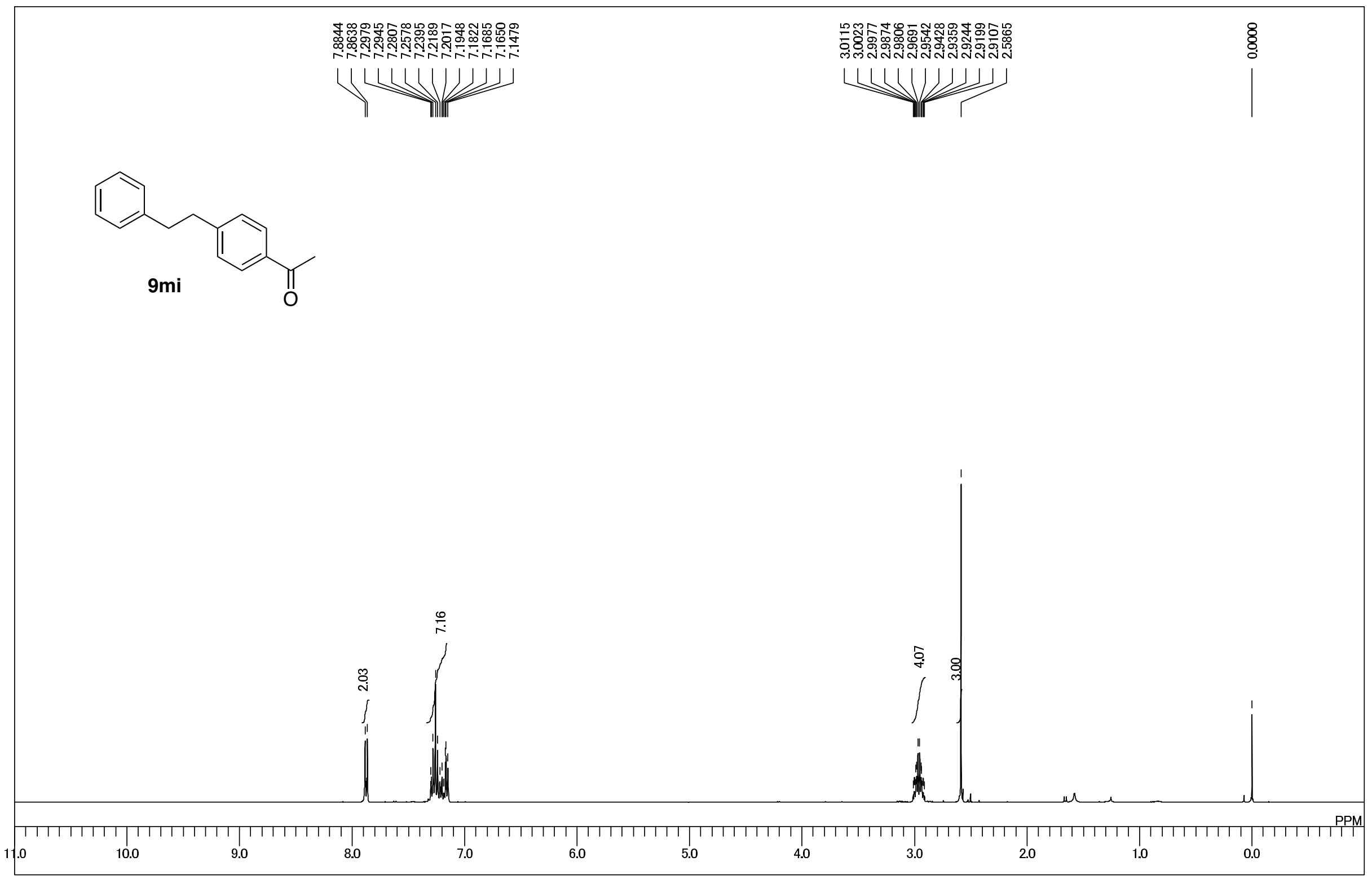

S87 


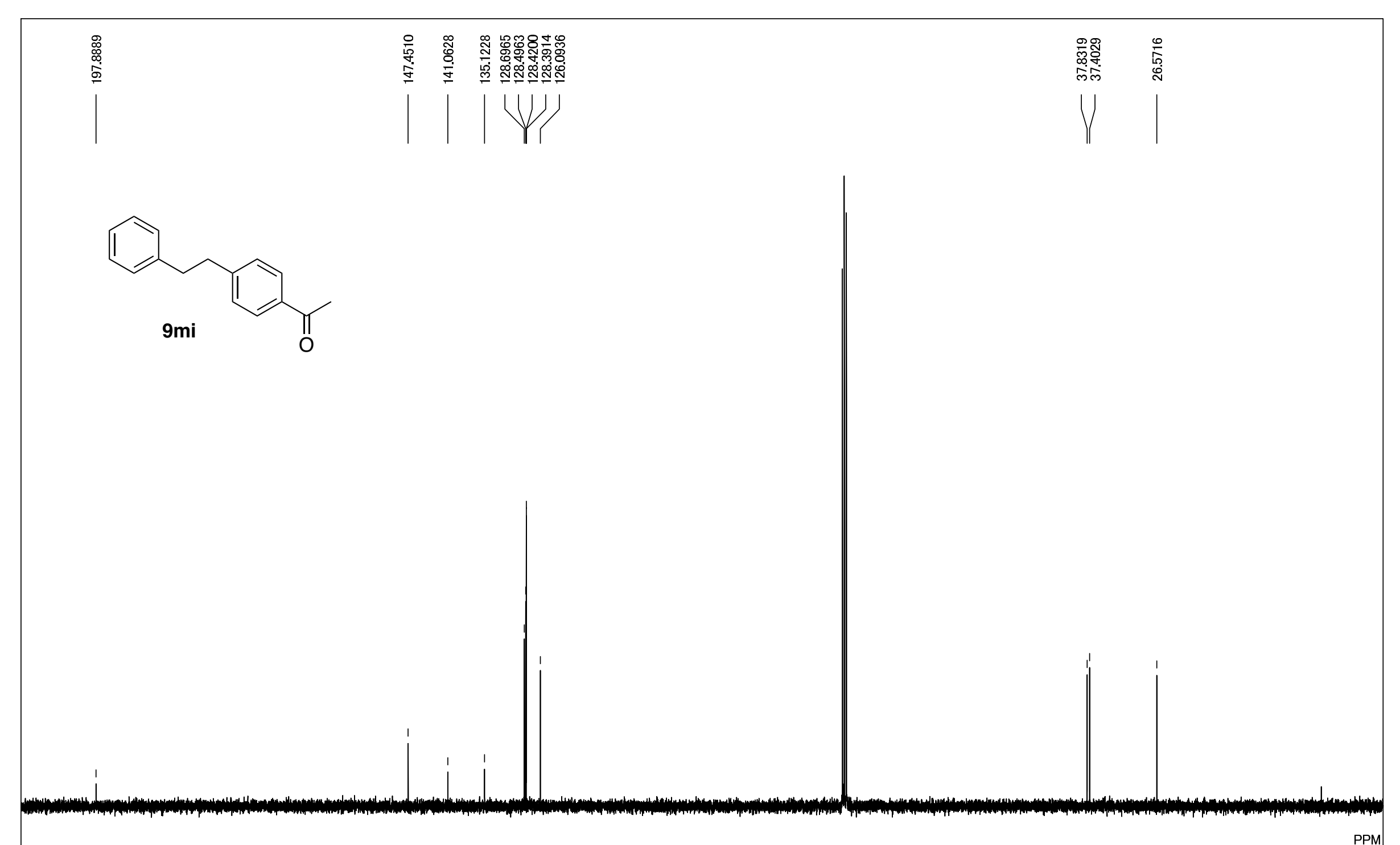




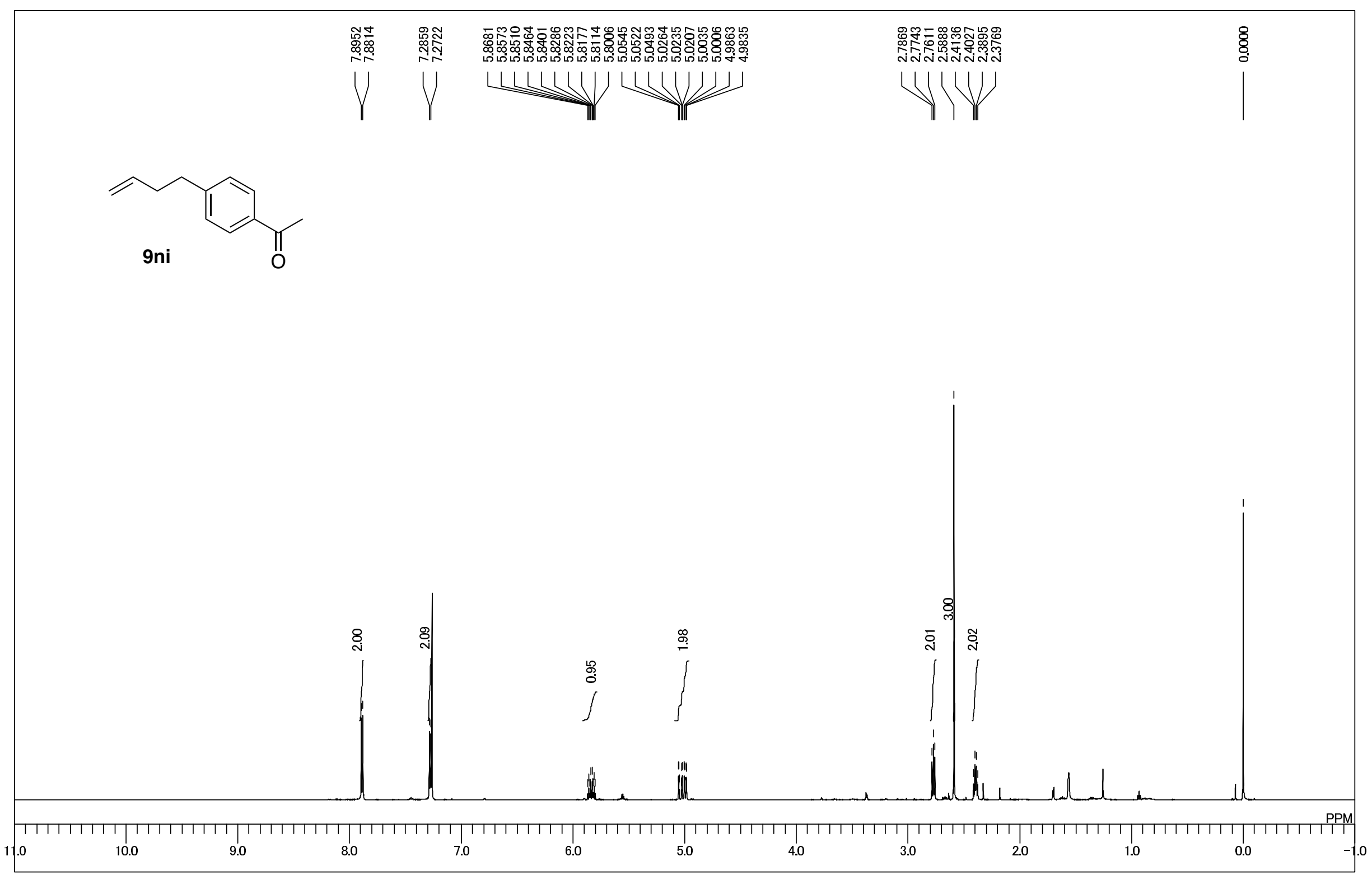

S89 


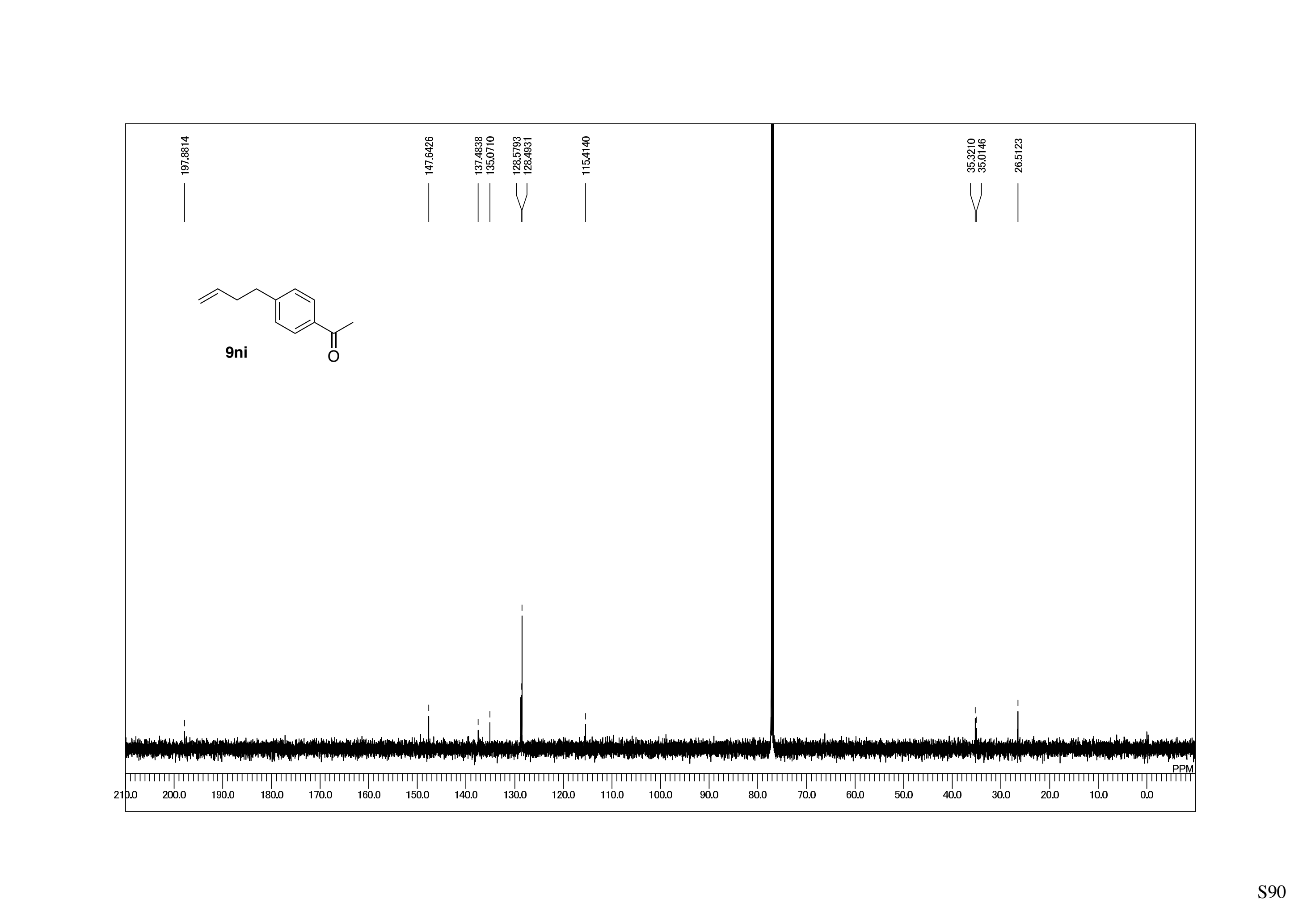




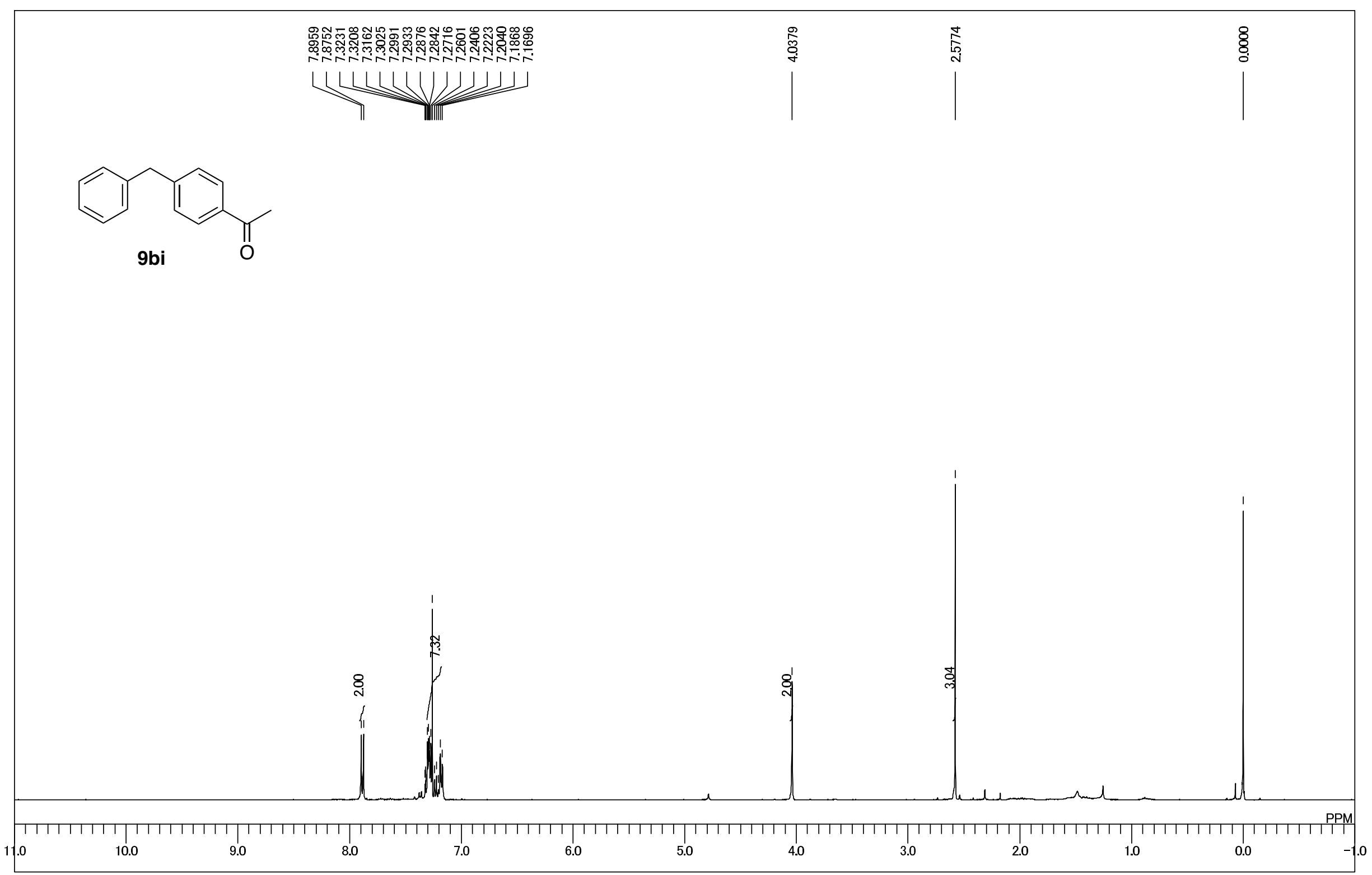

S91 


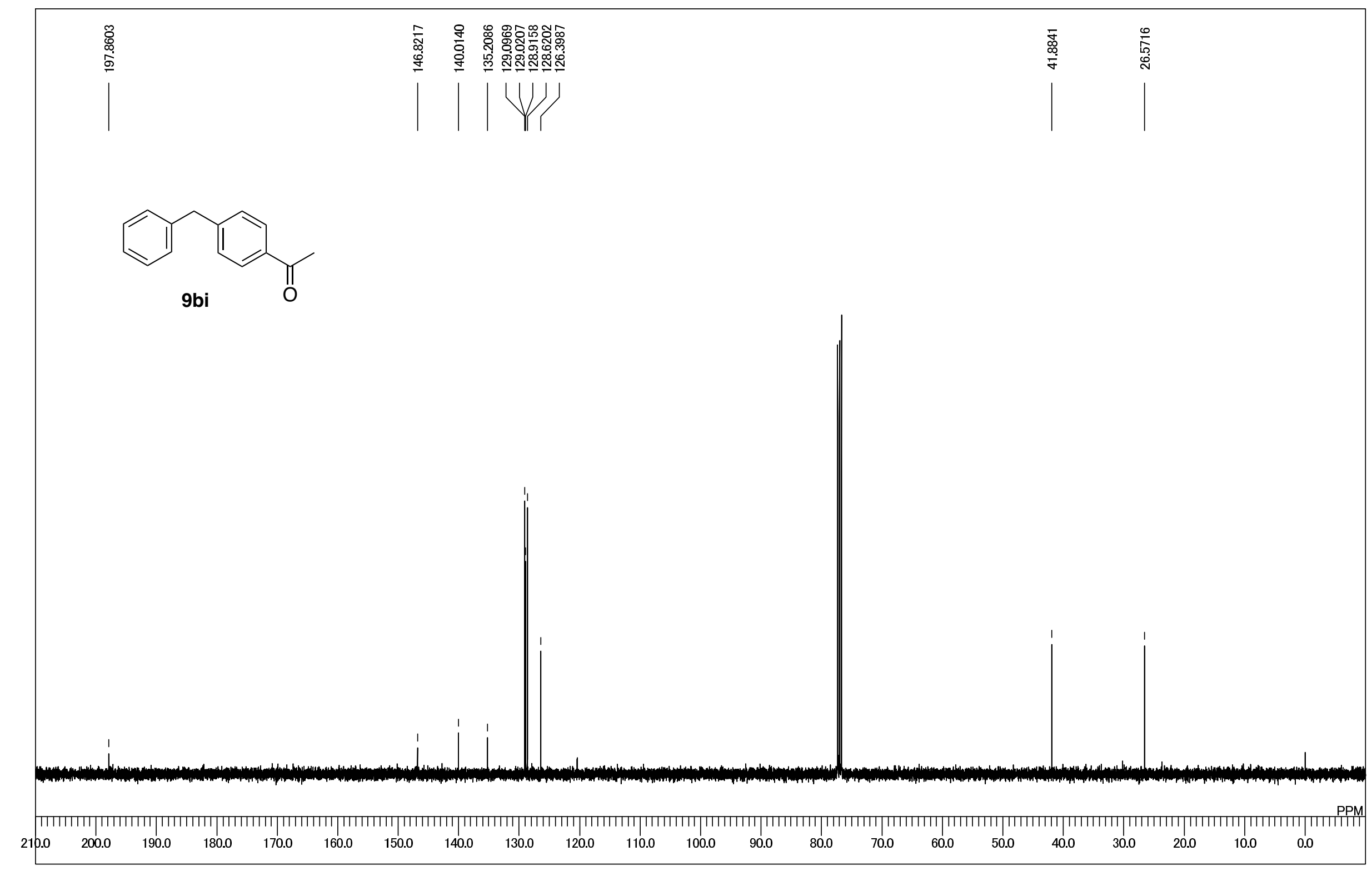

S92 


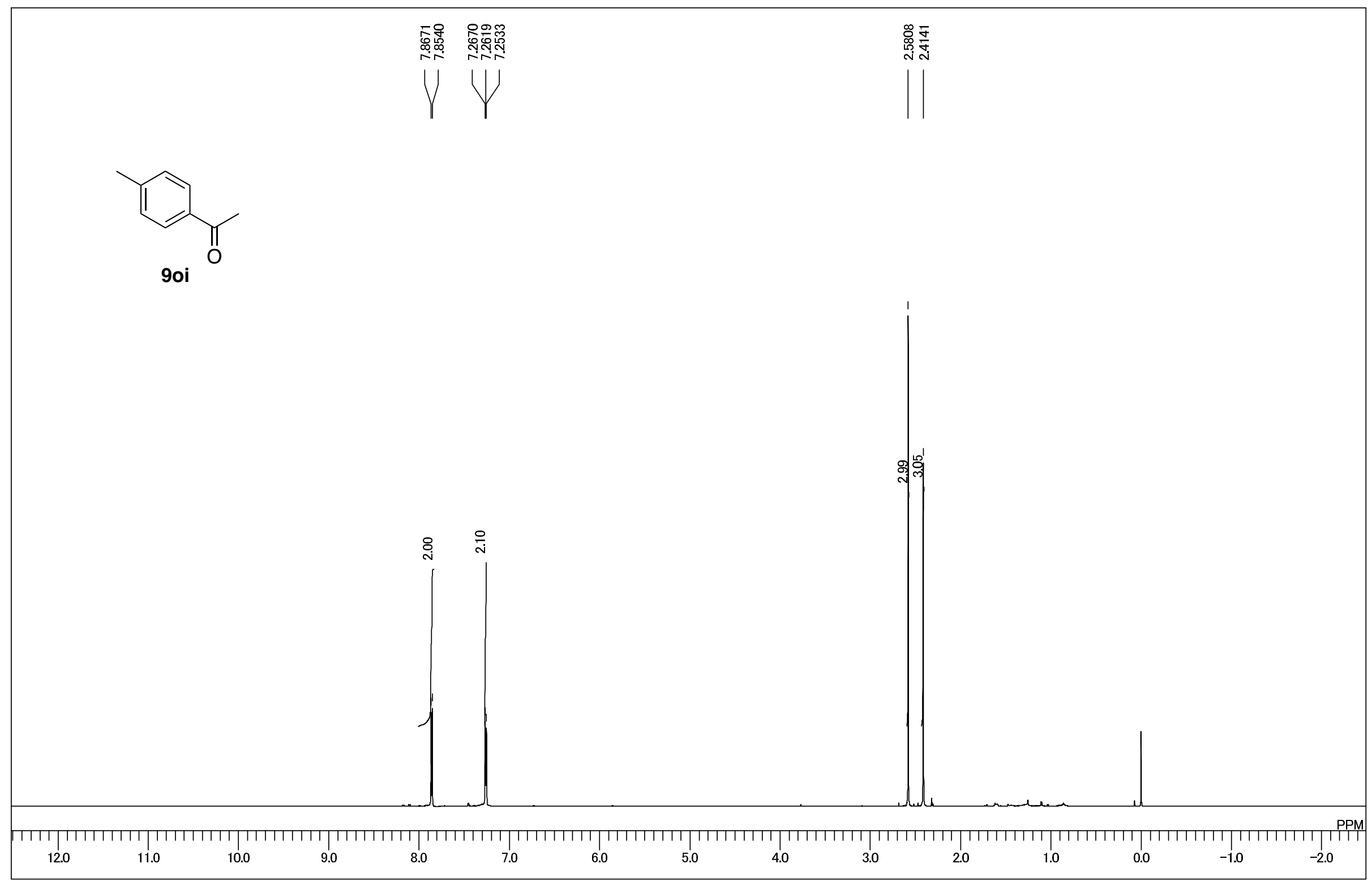




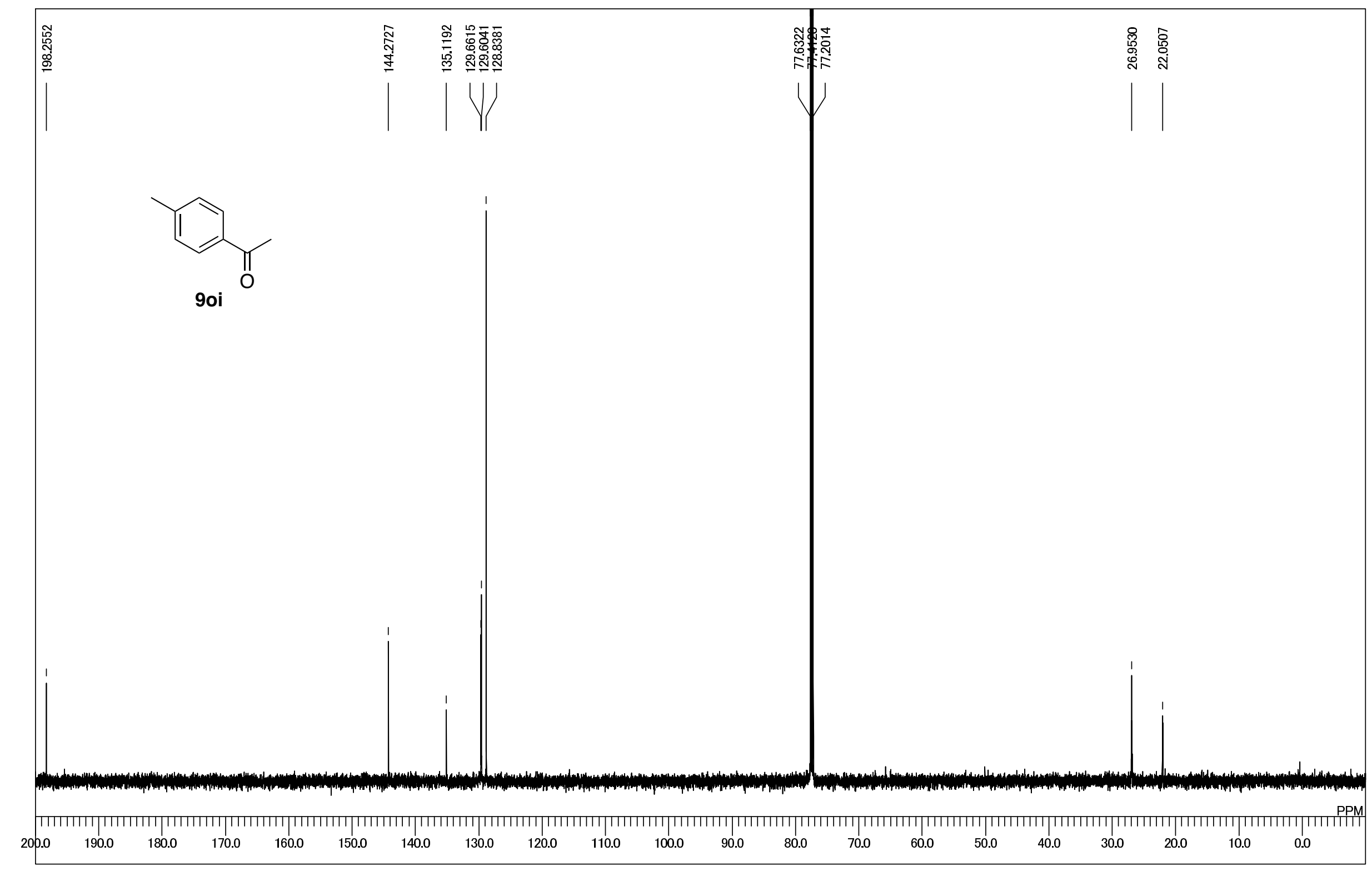

S94 


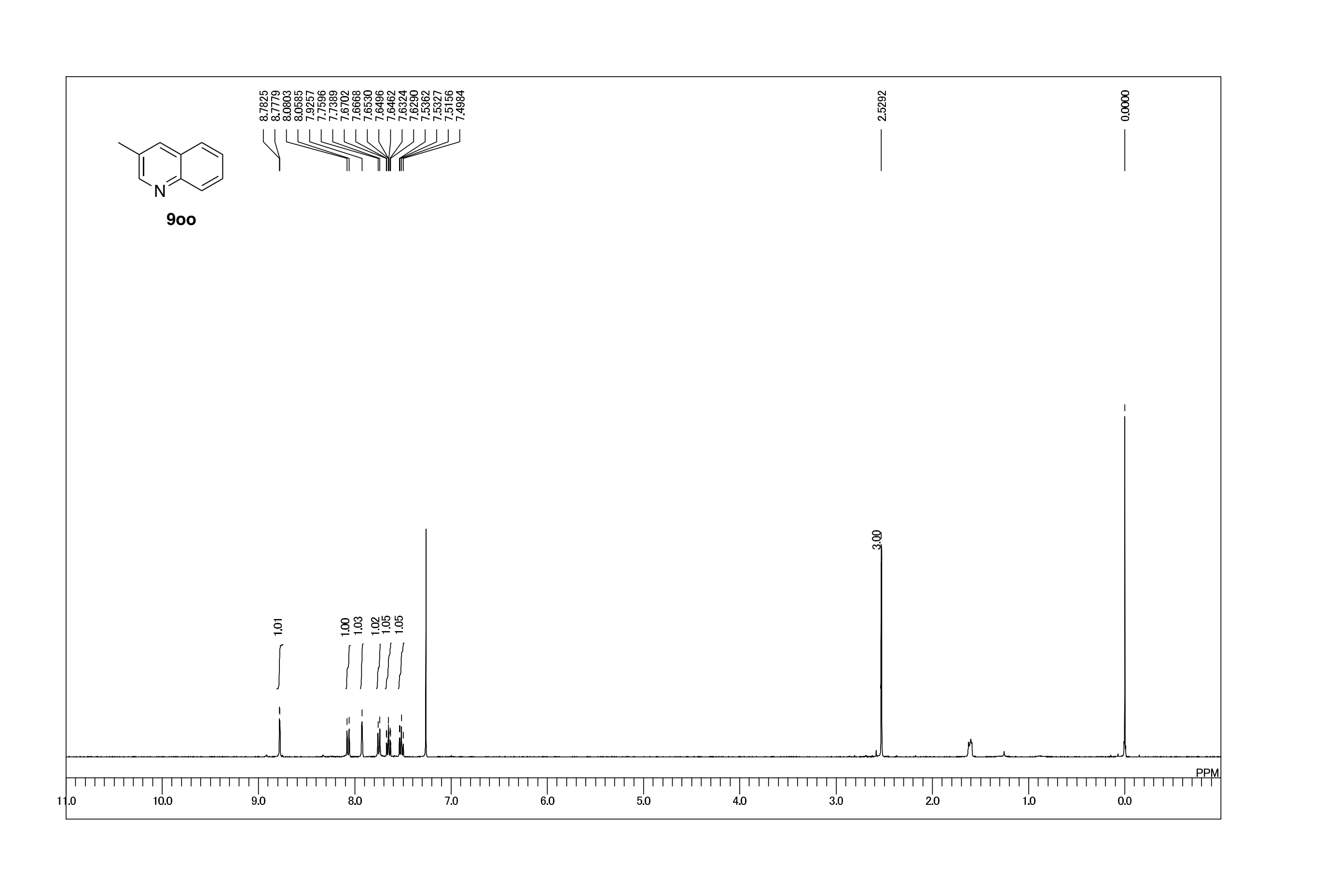




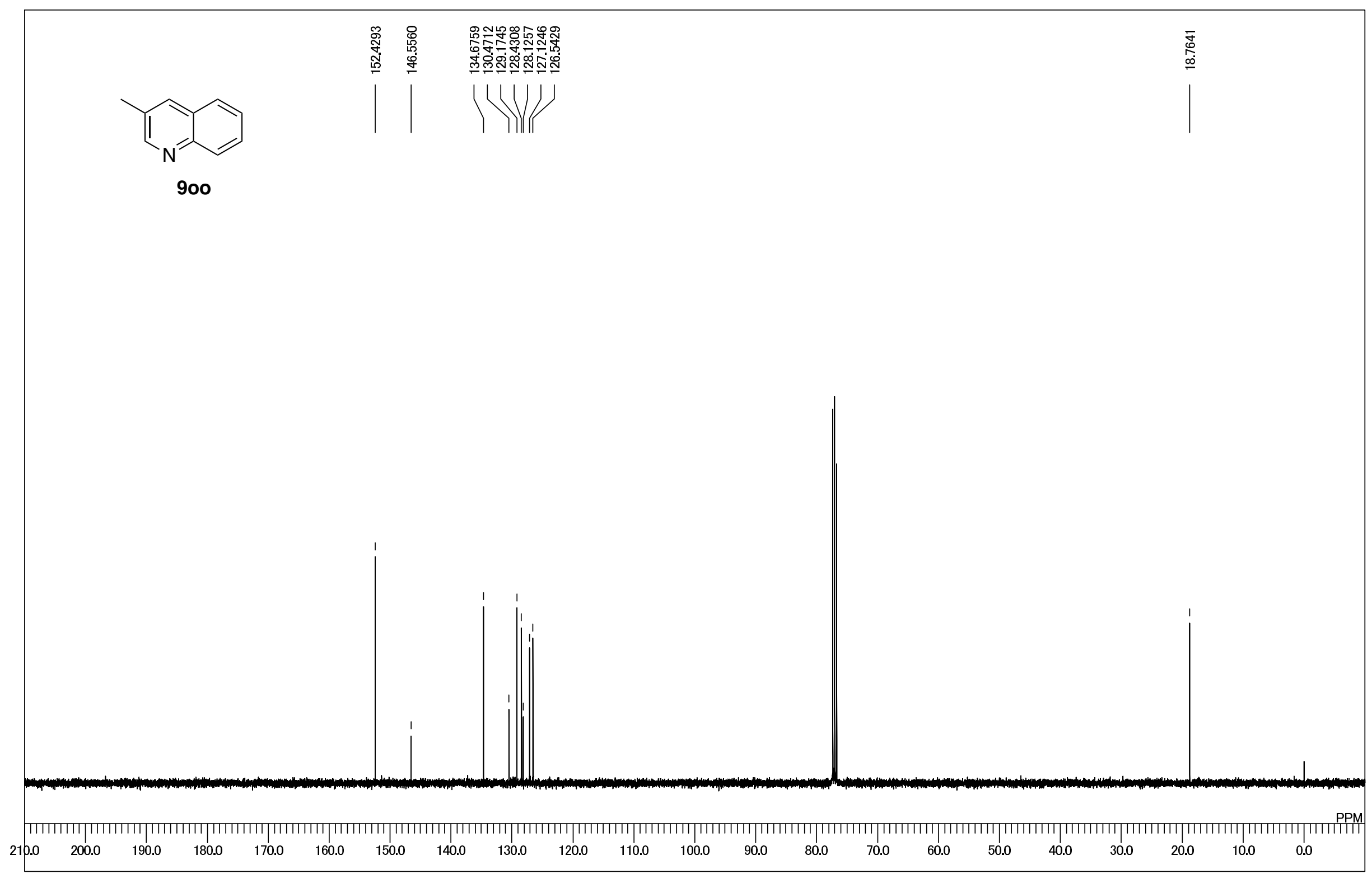




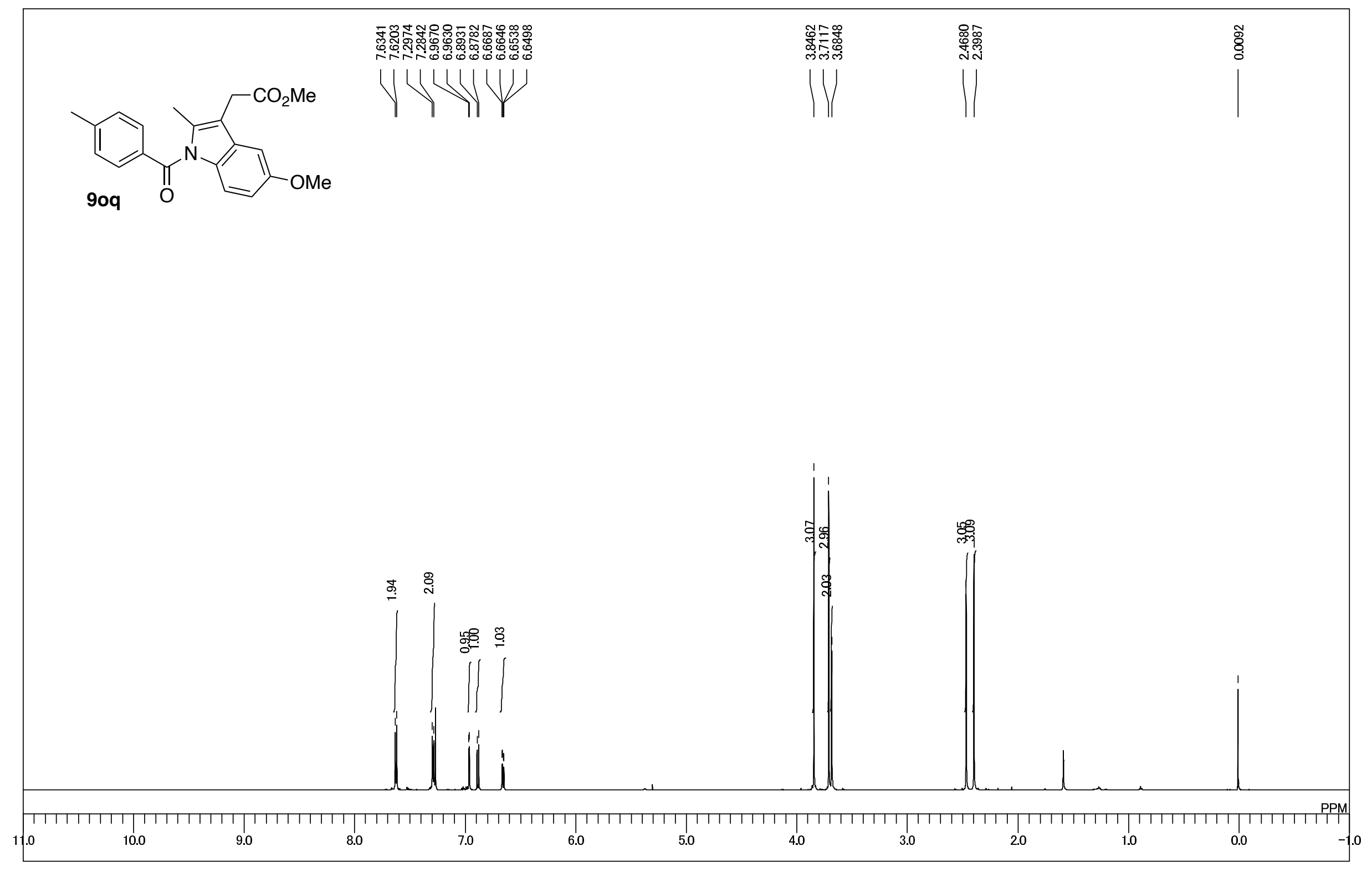




$$
+1
$$




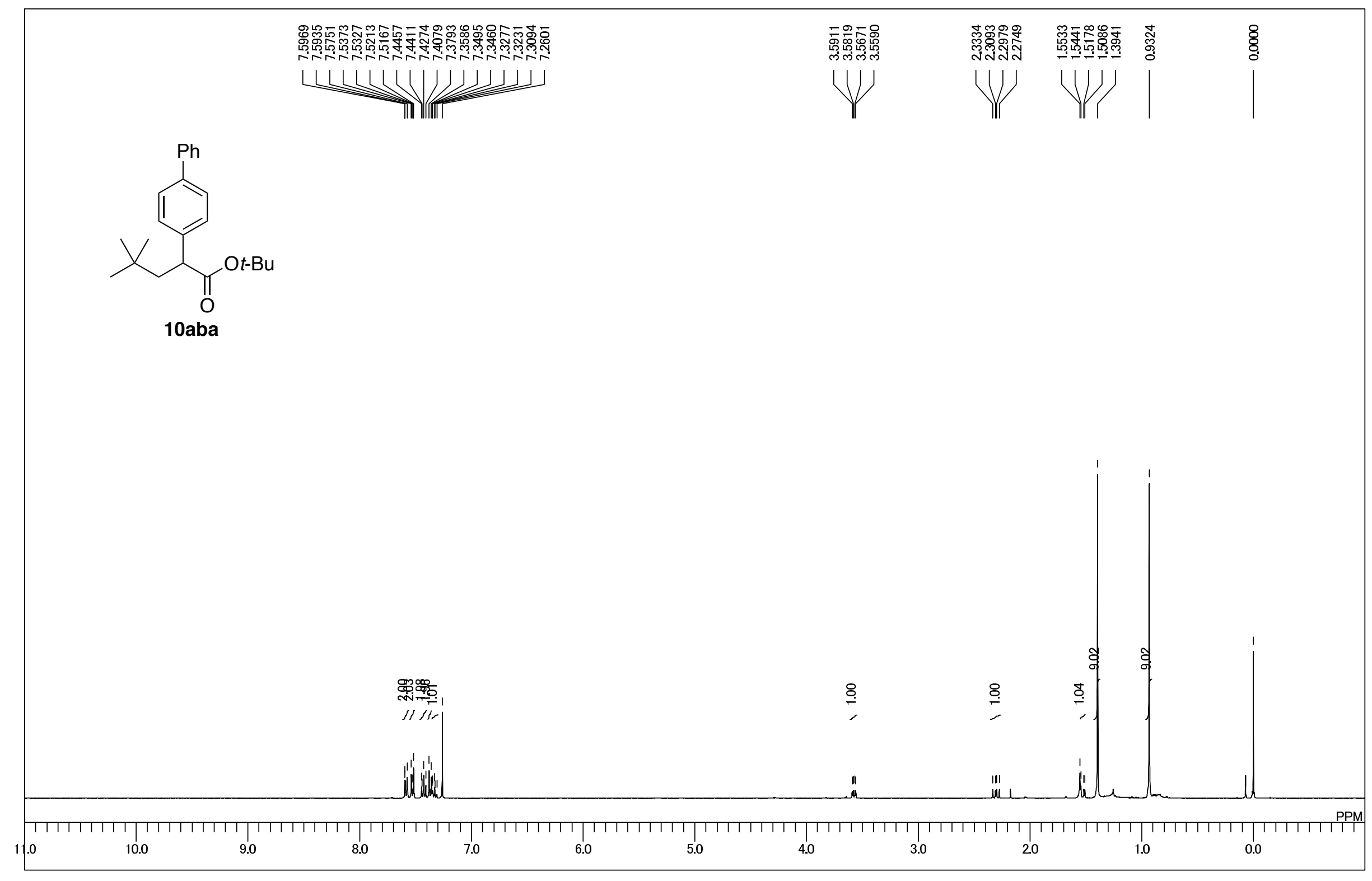




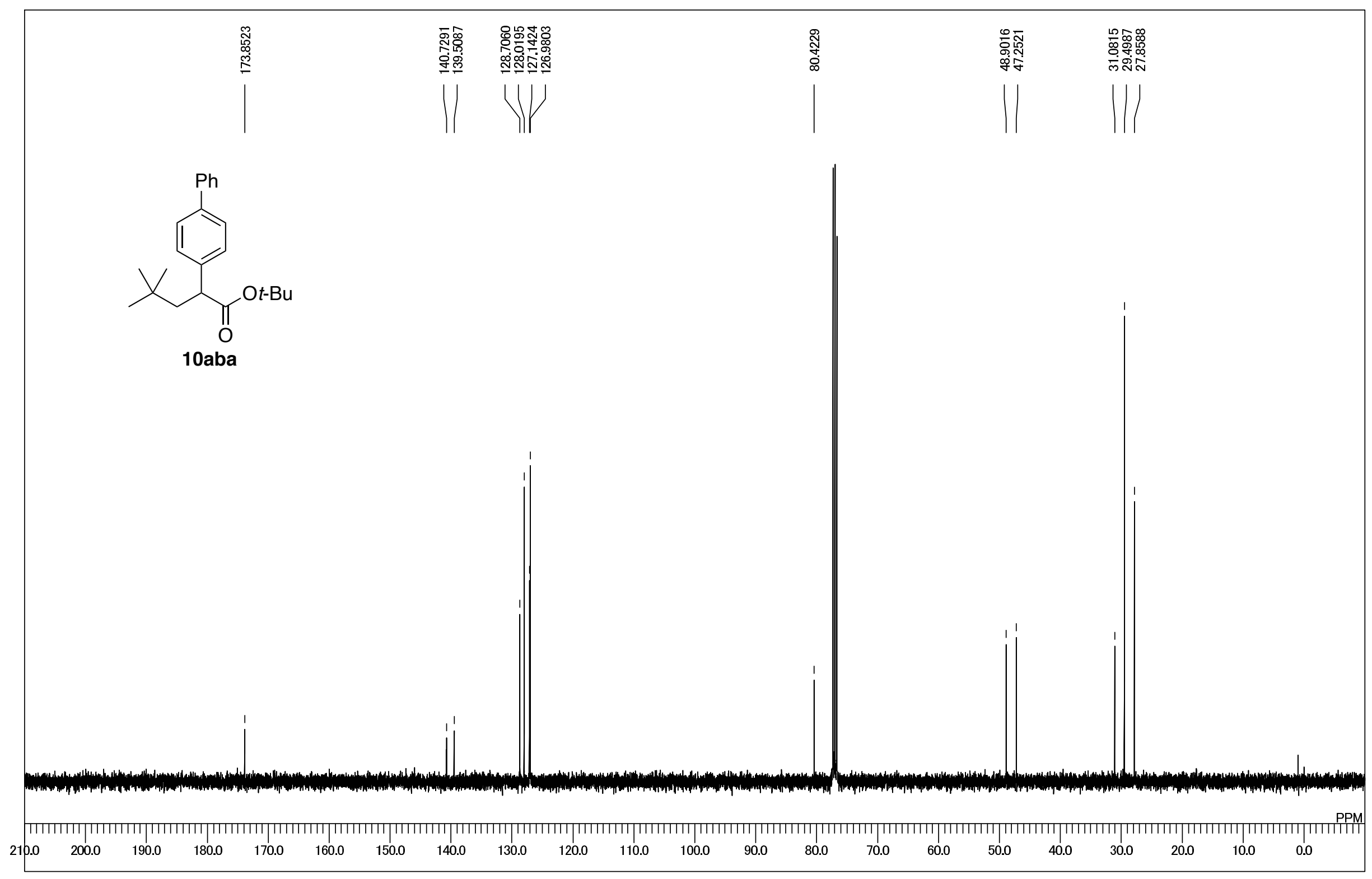




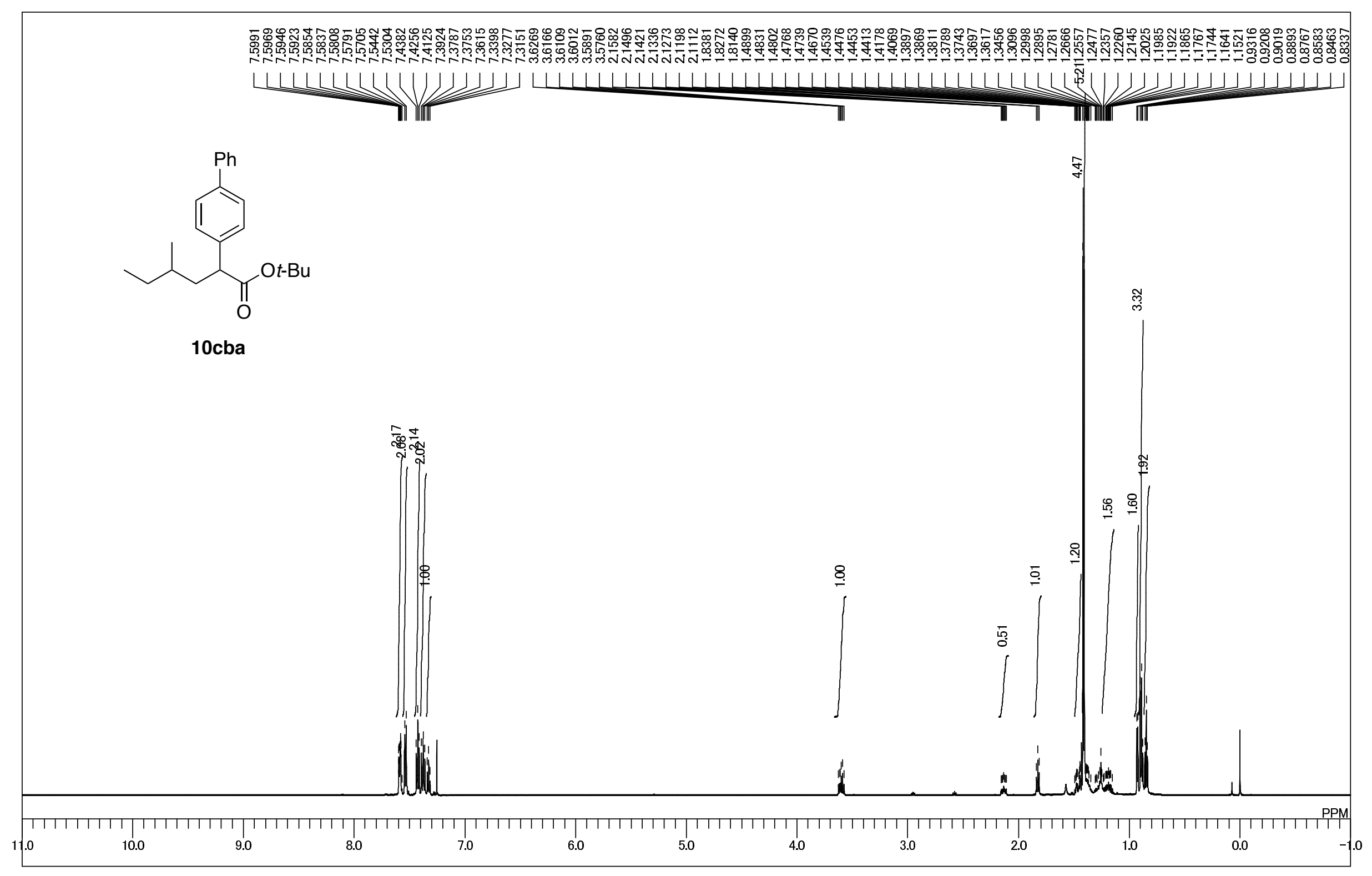




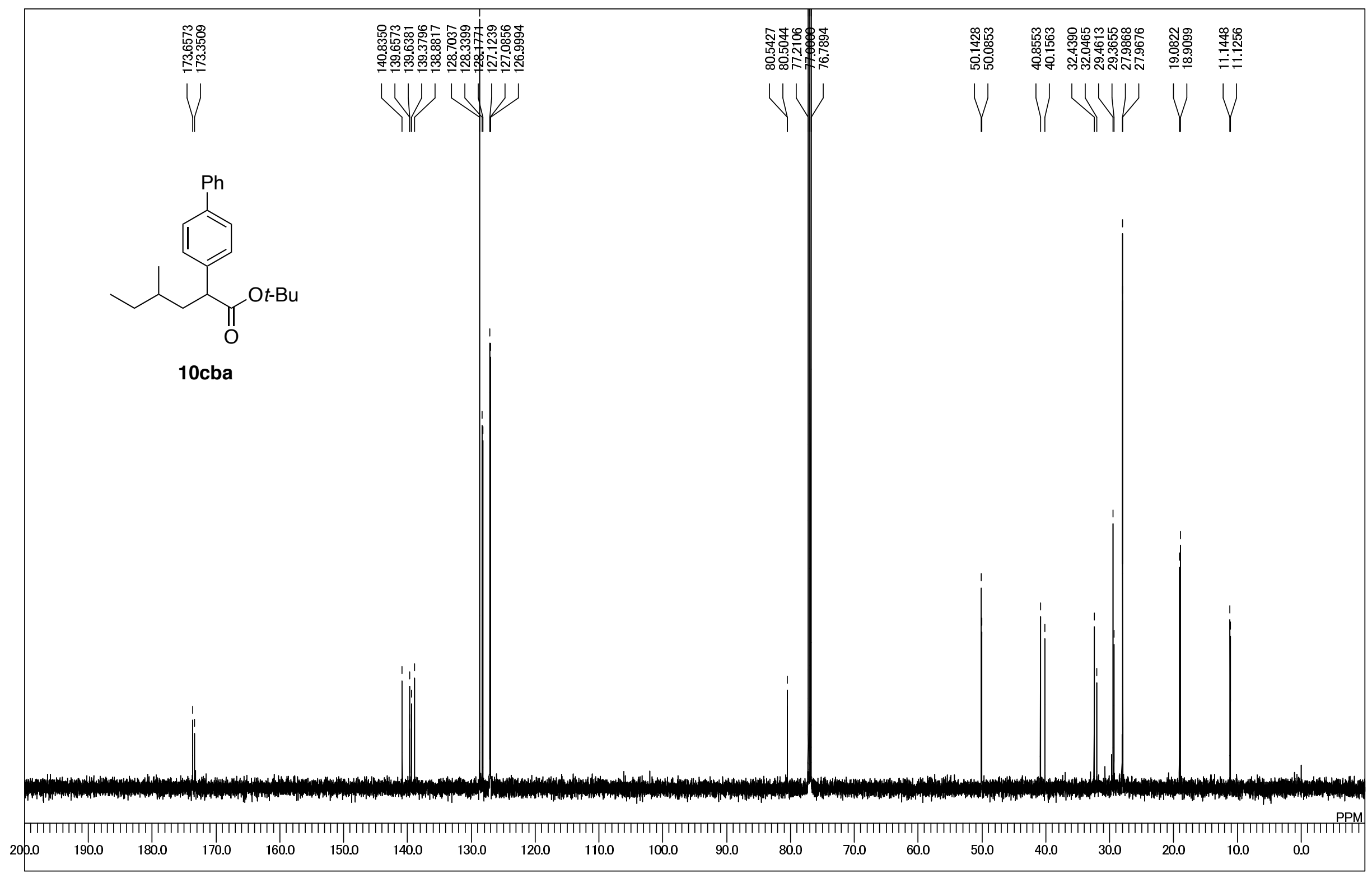




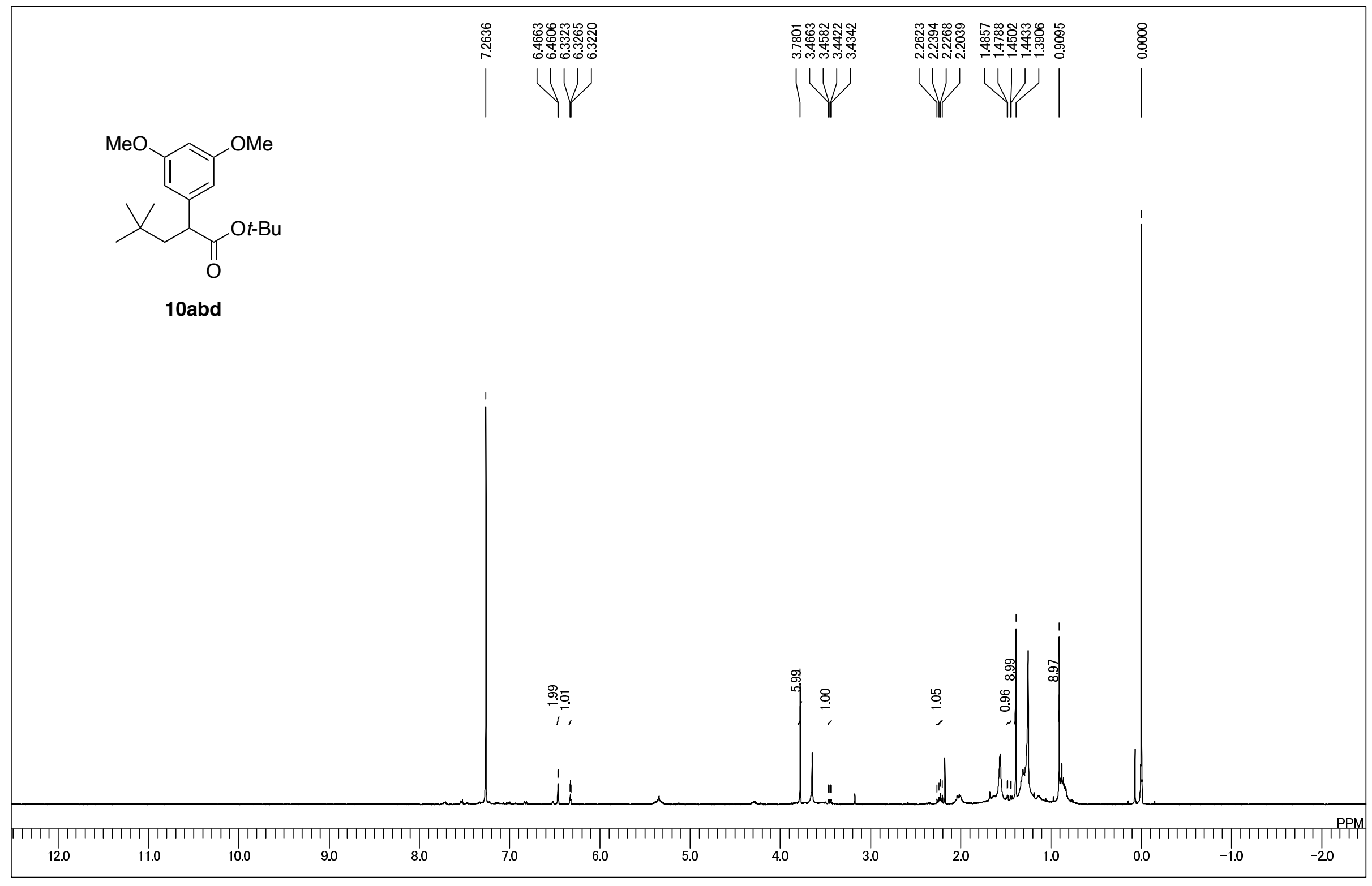




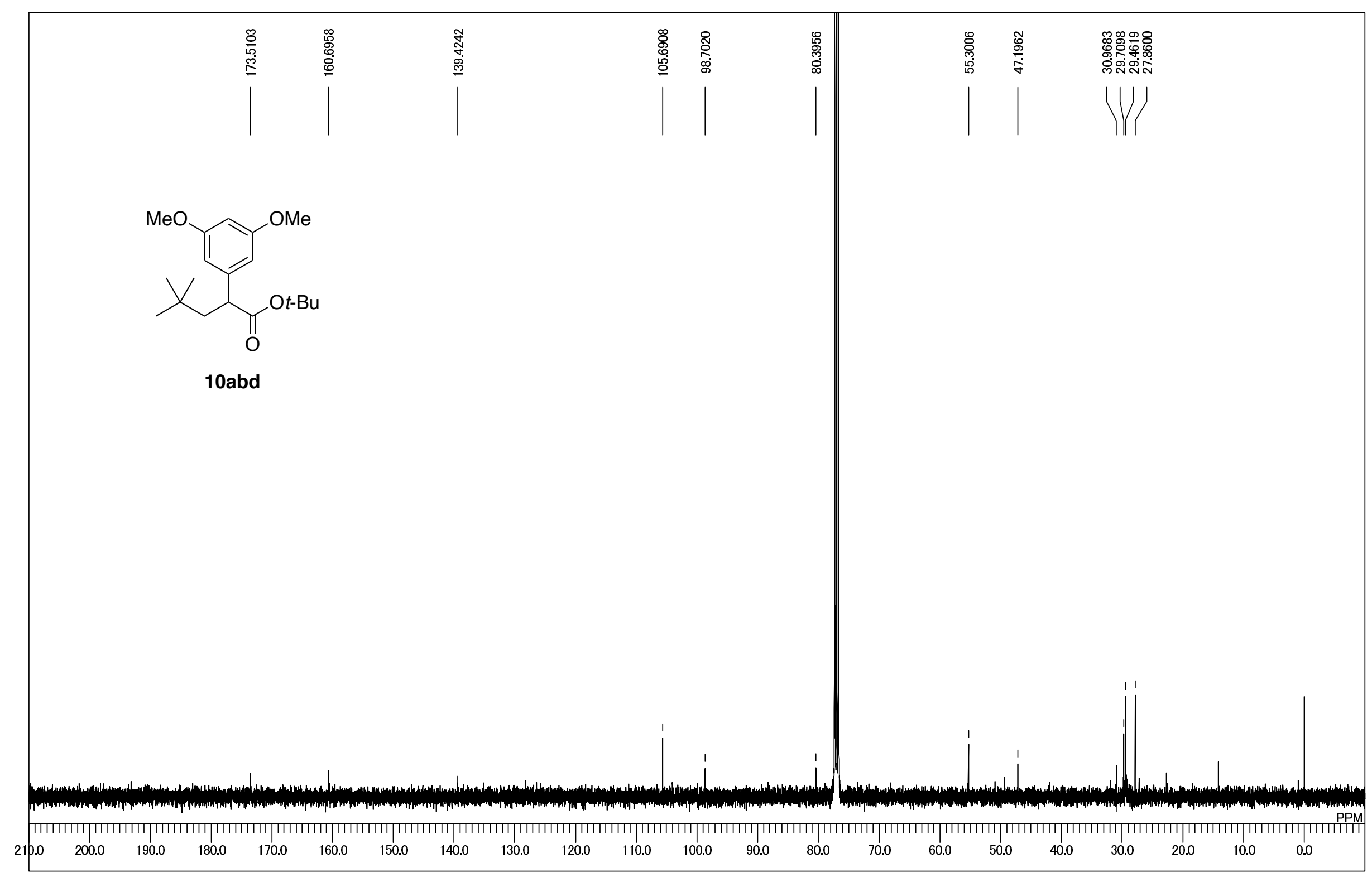




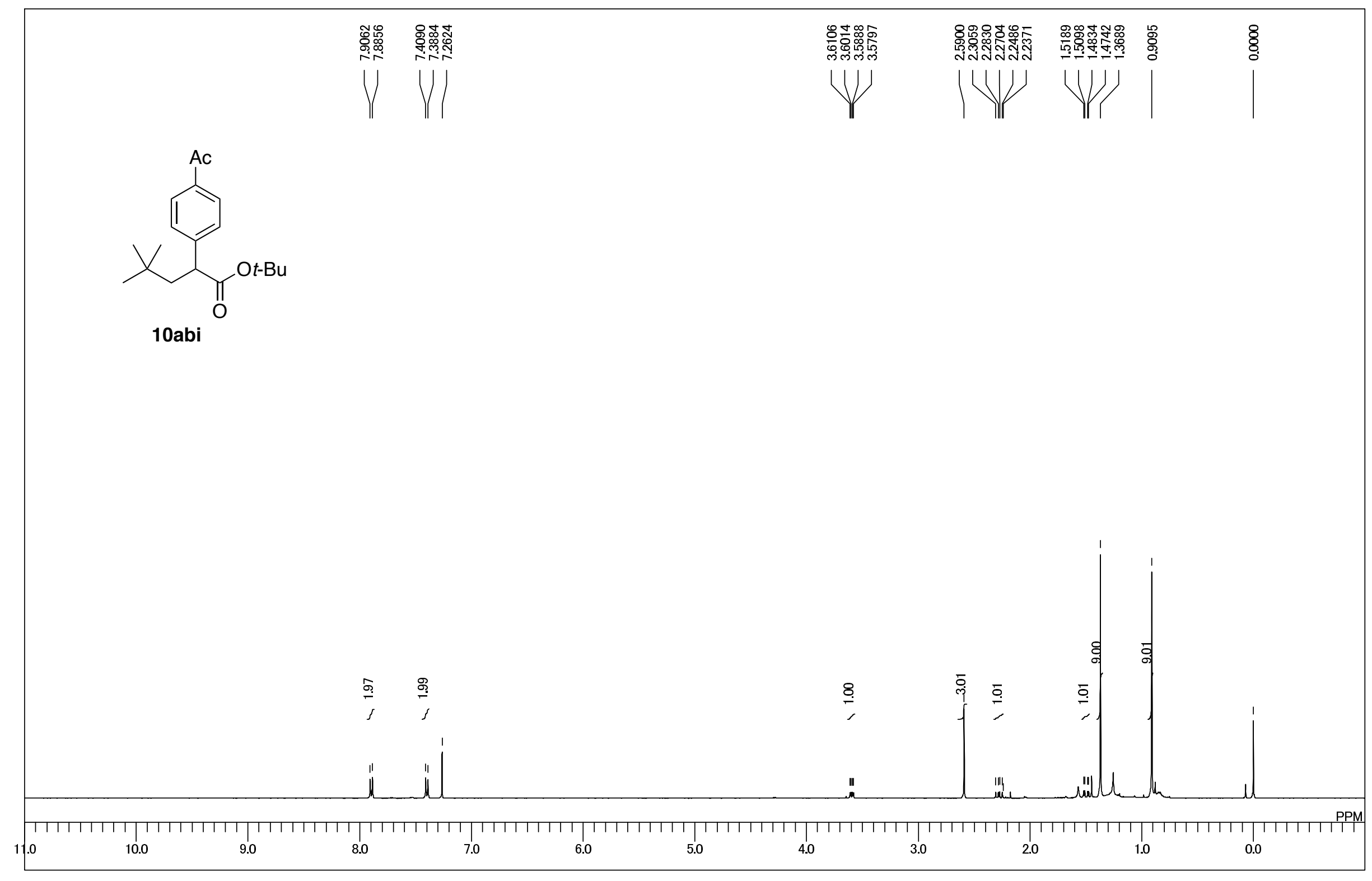




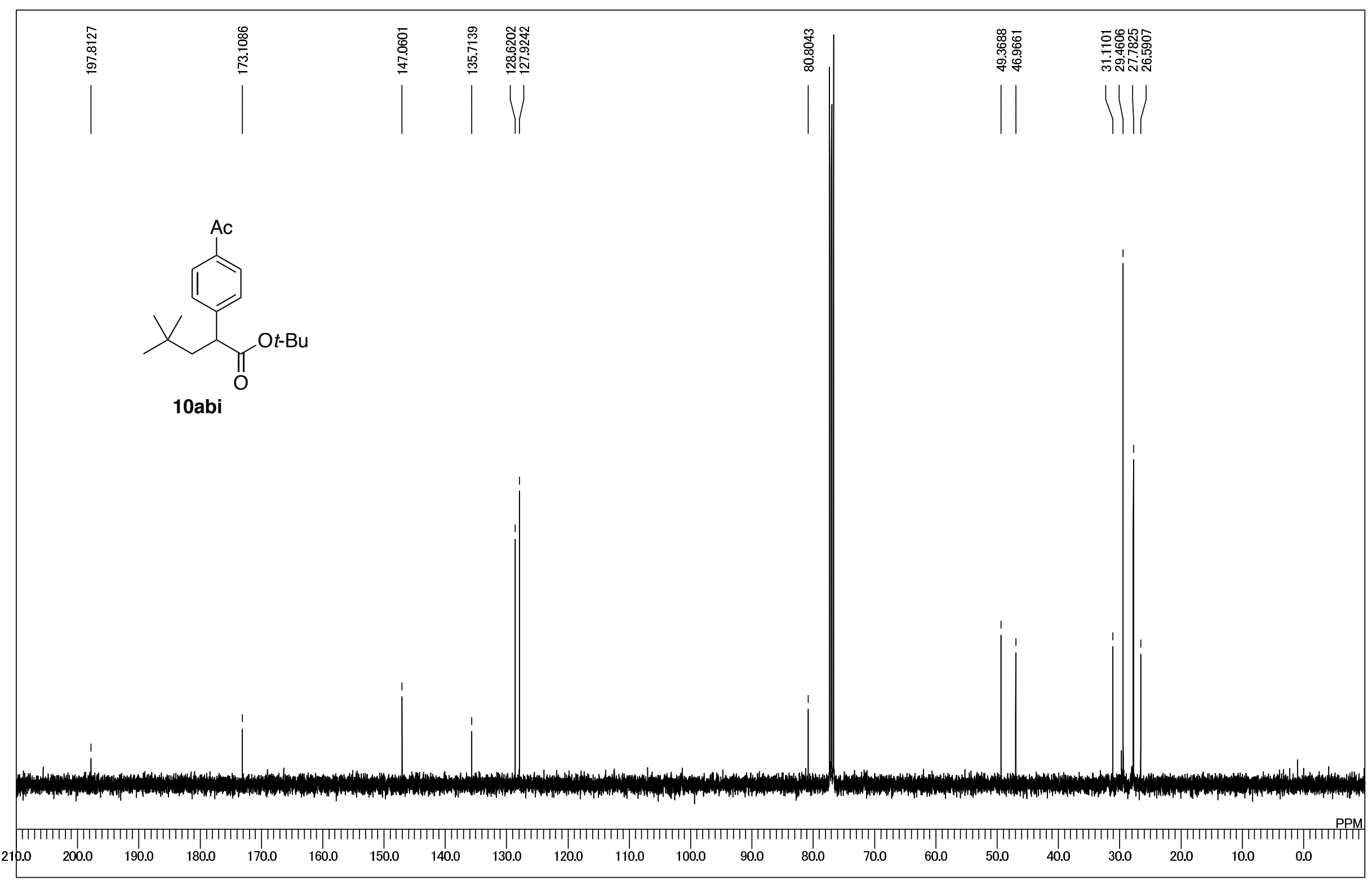




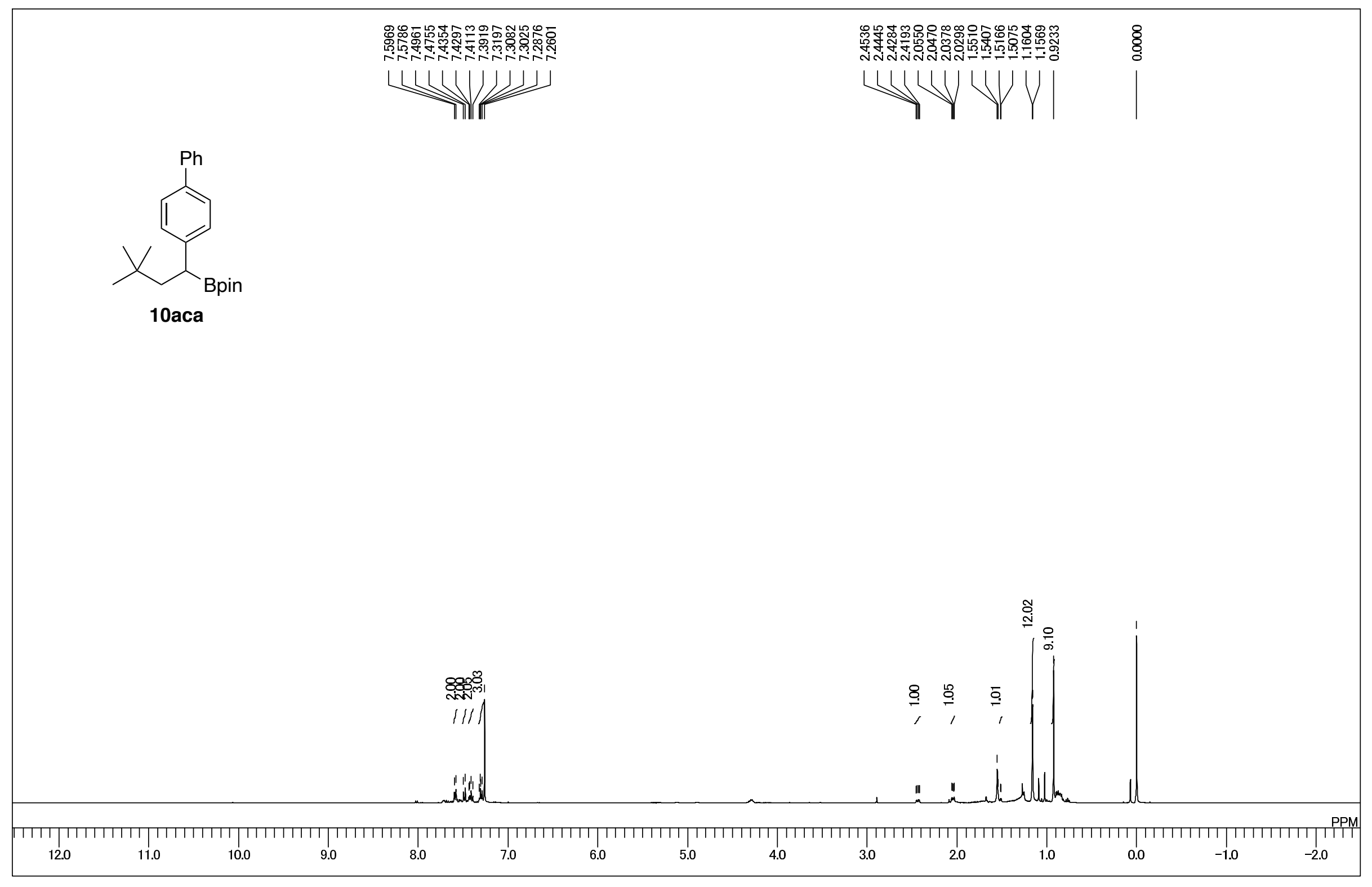




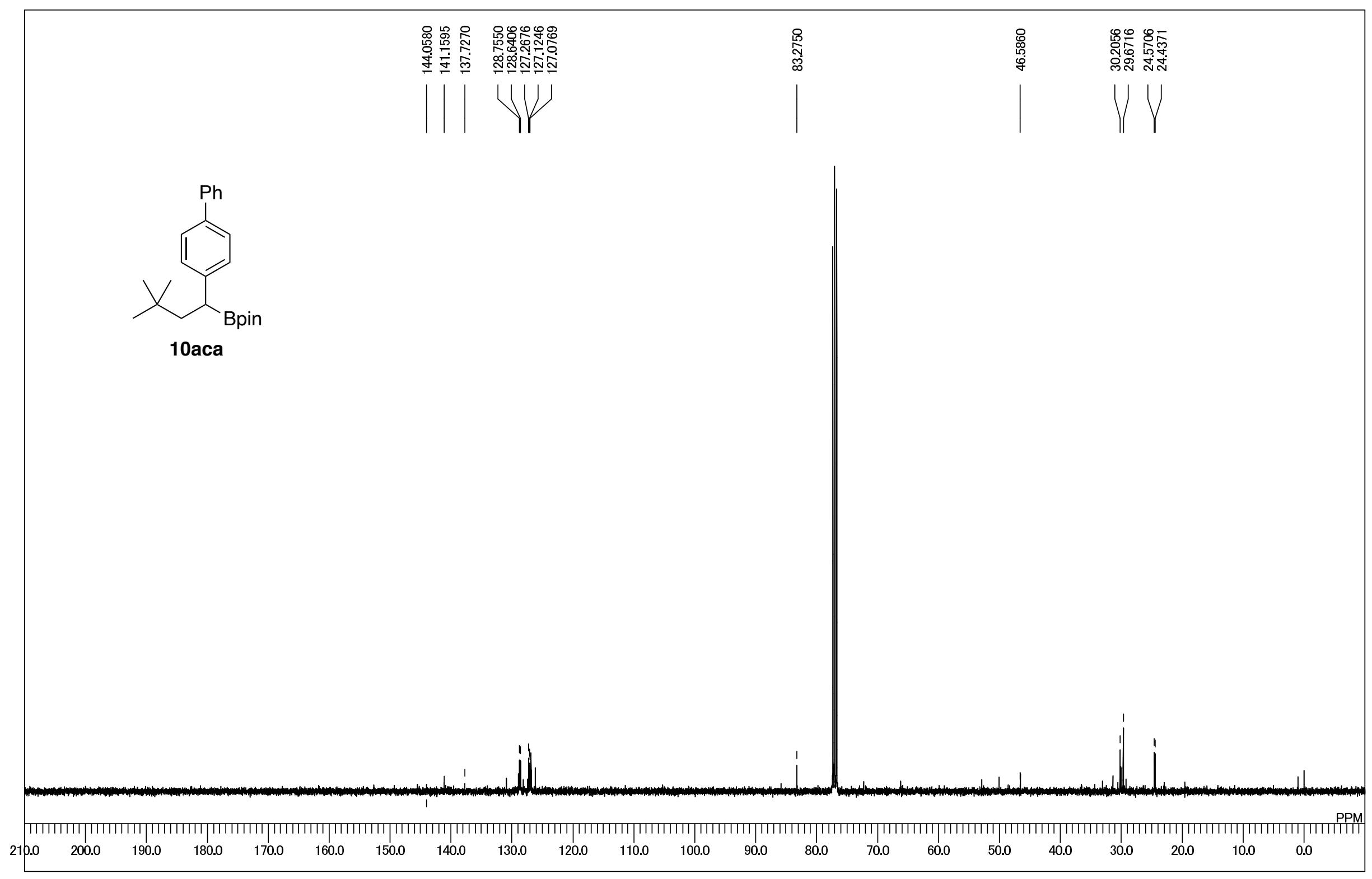




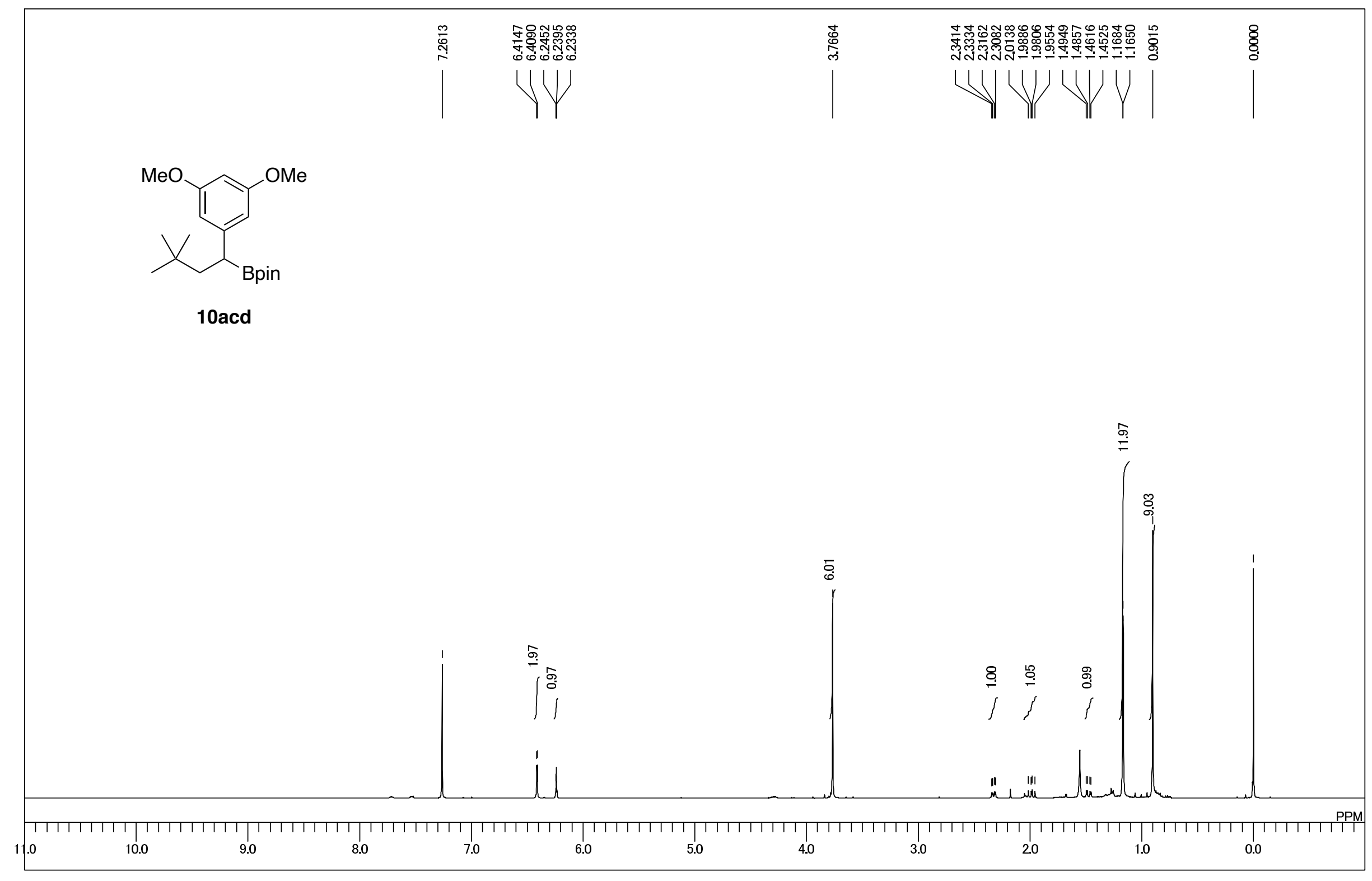




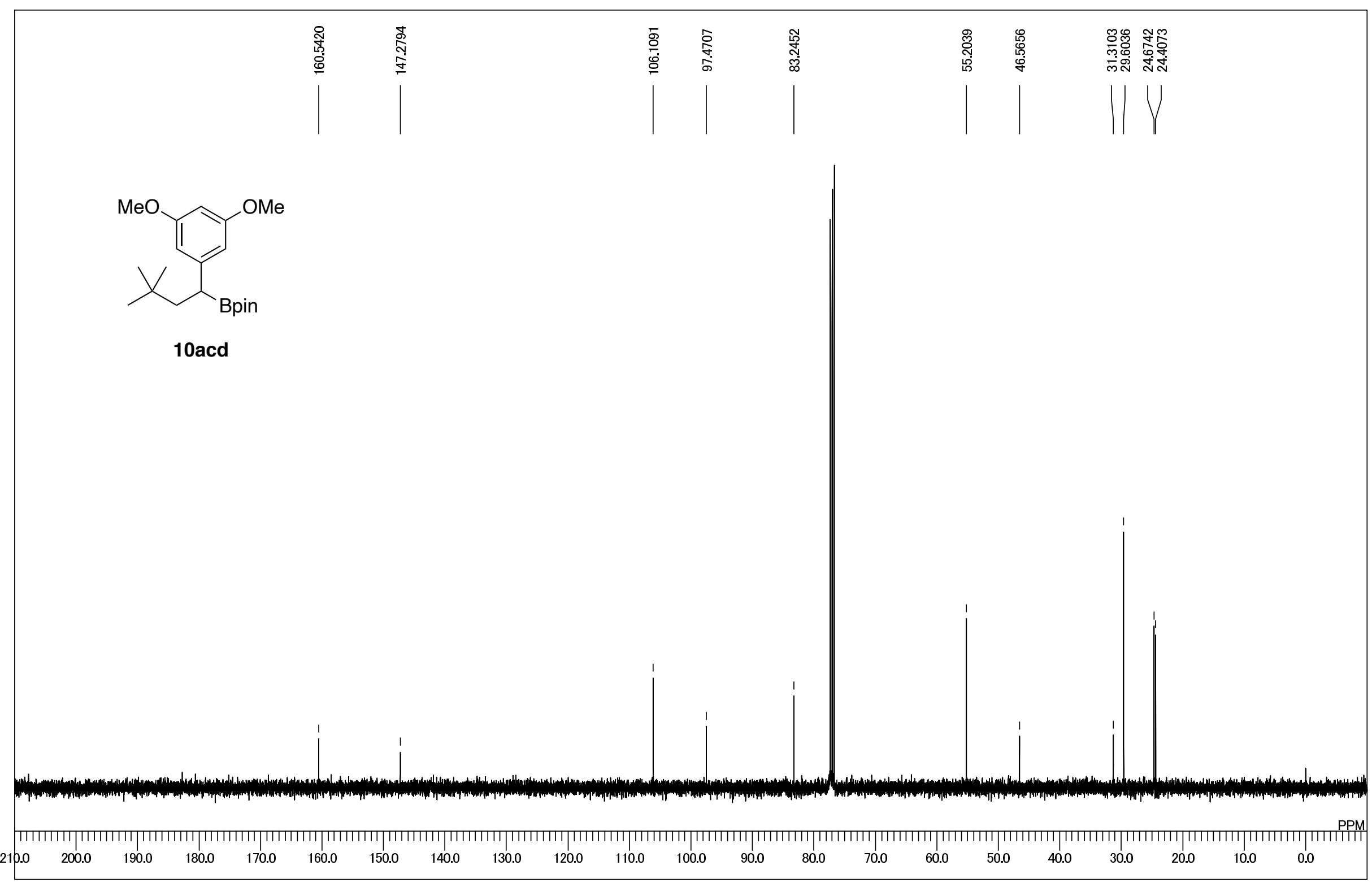




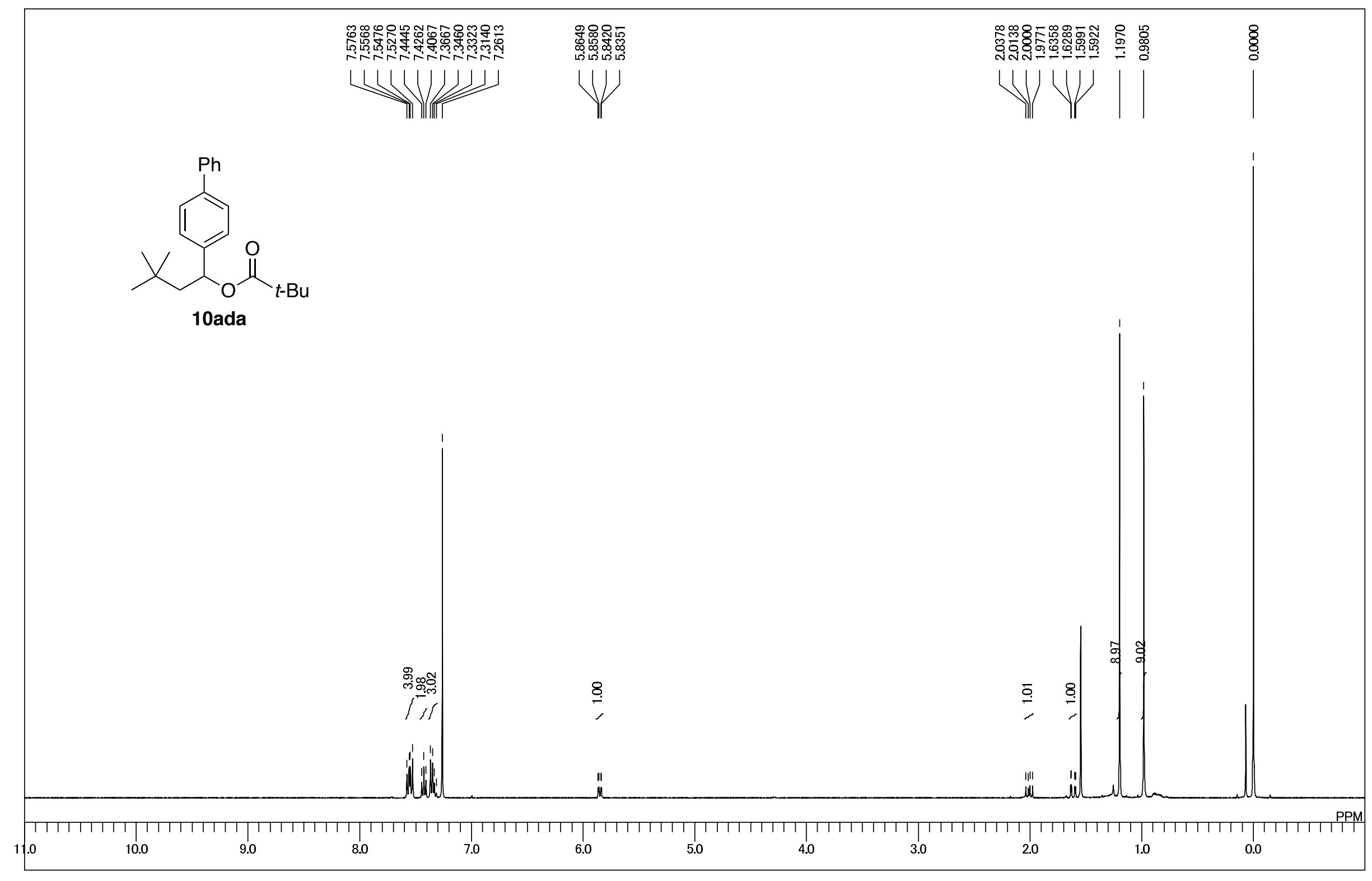




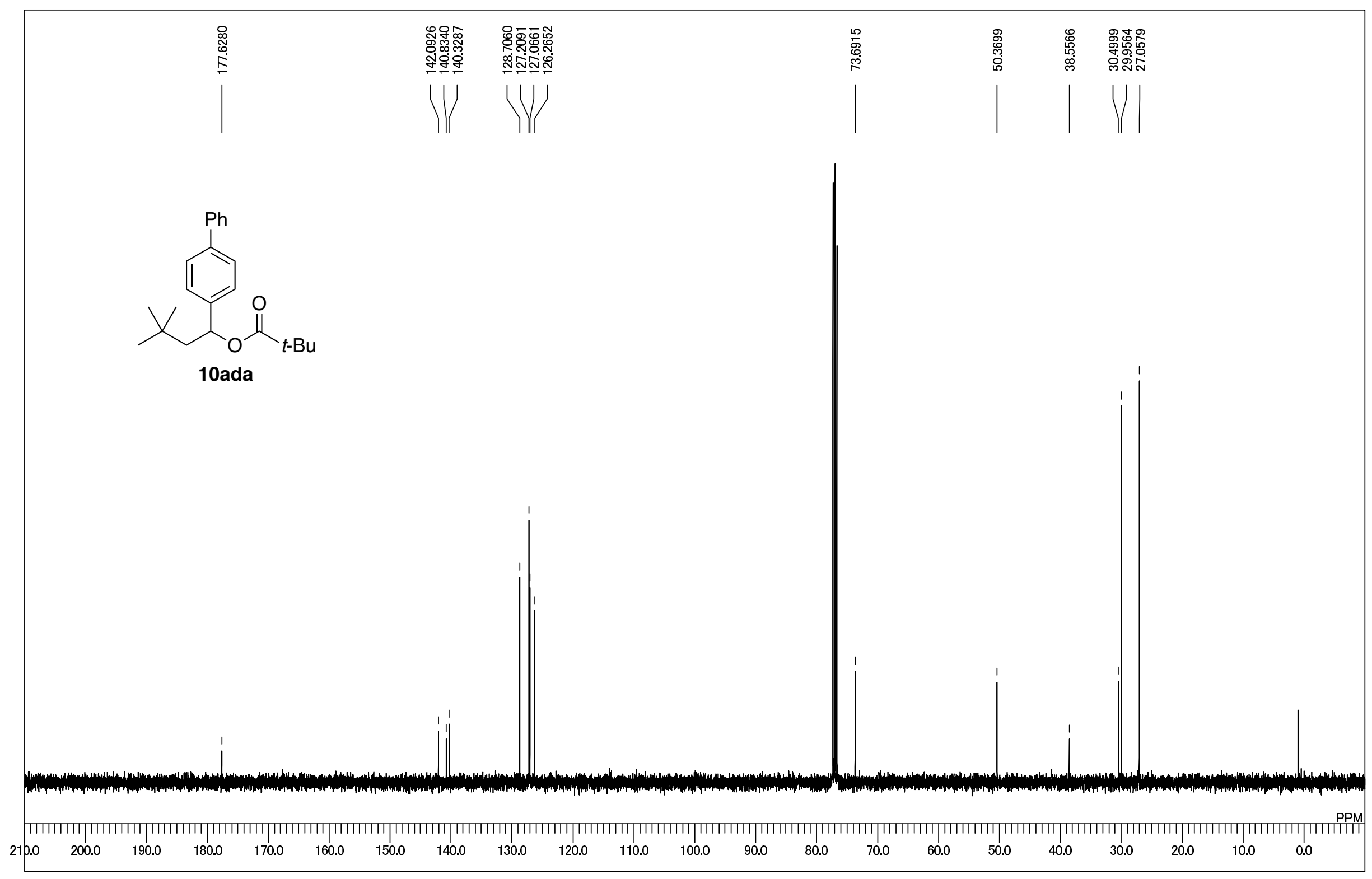




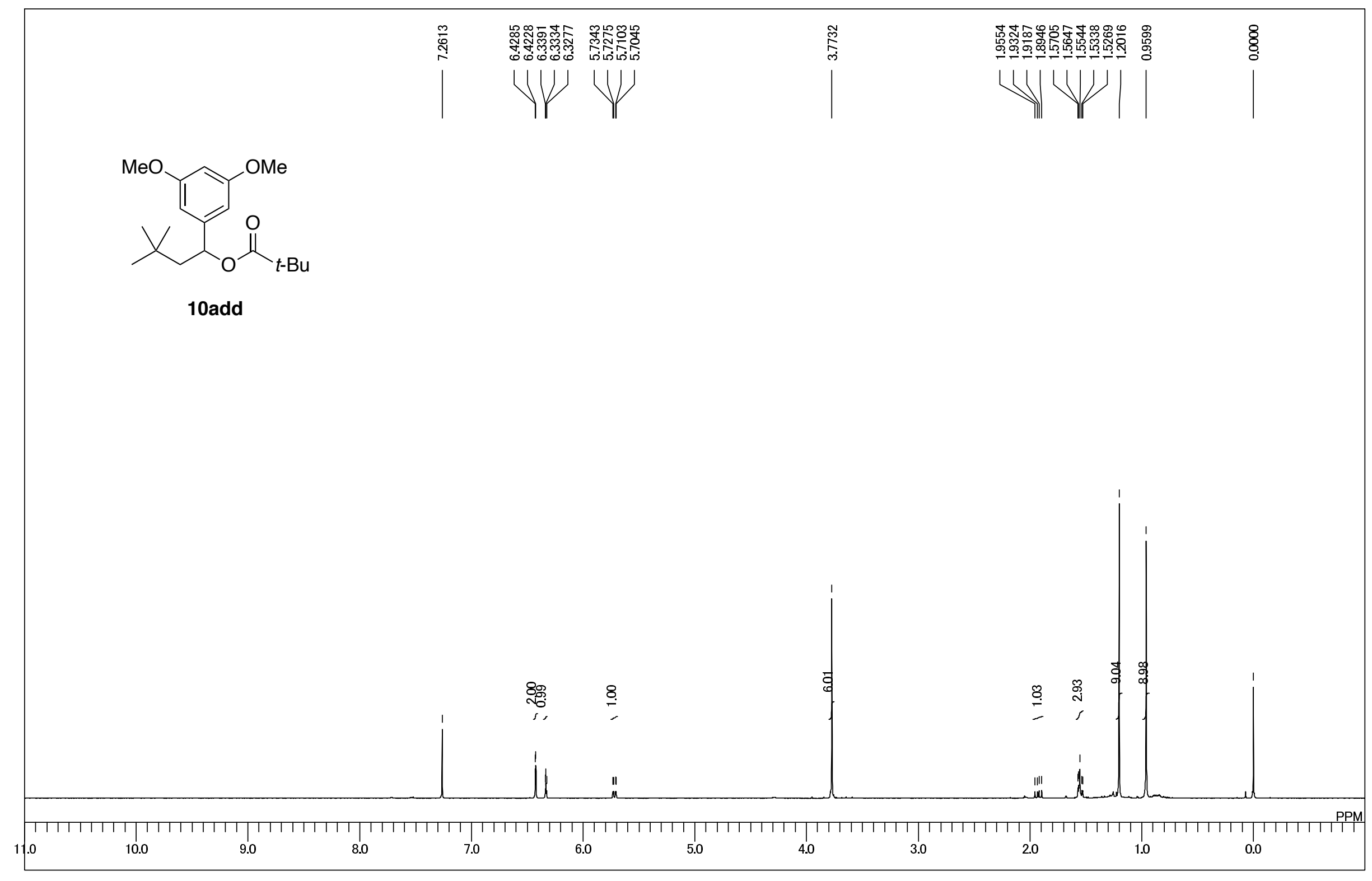




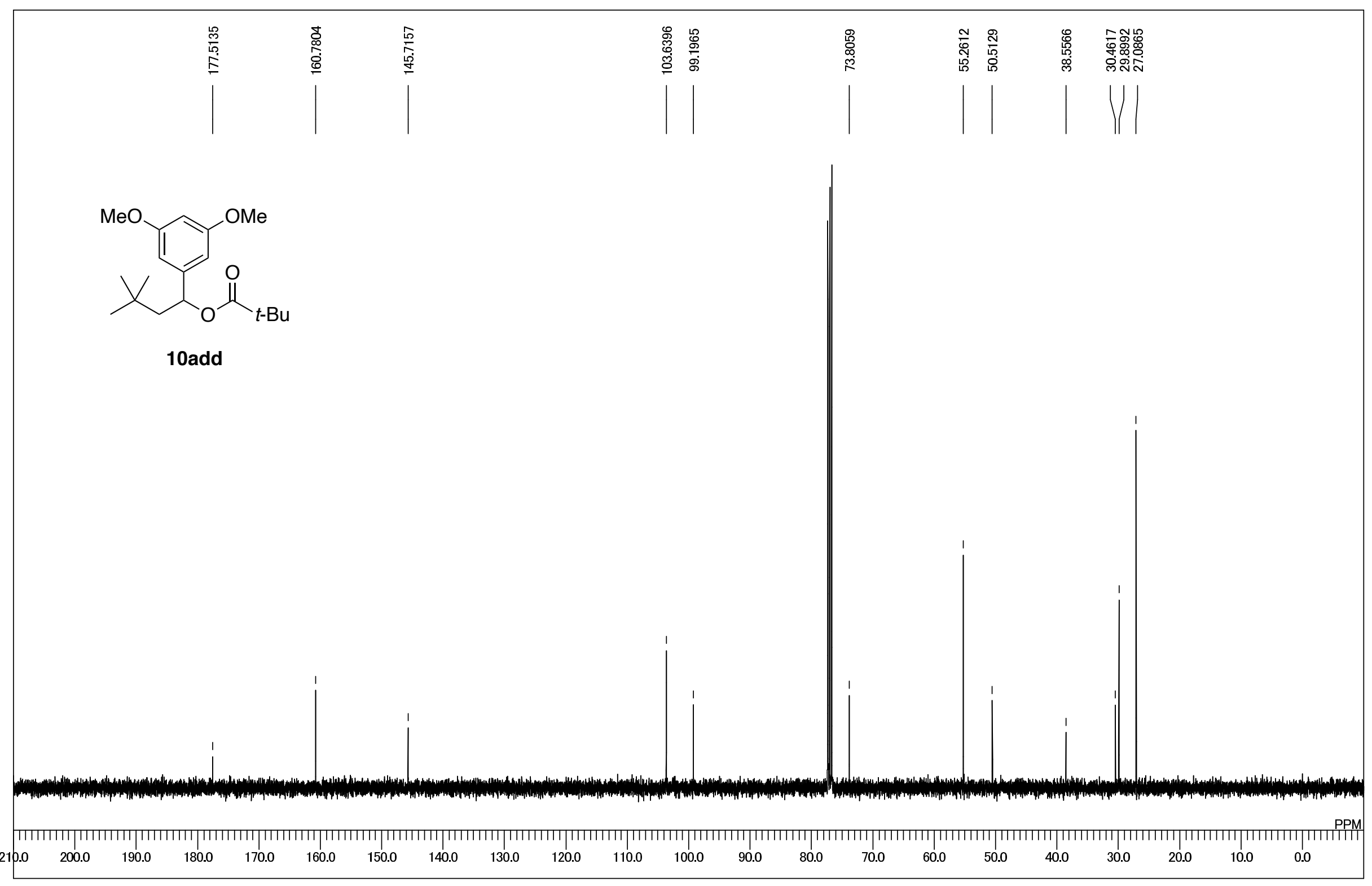




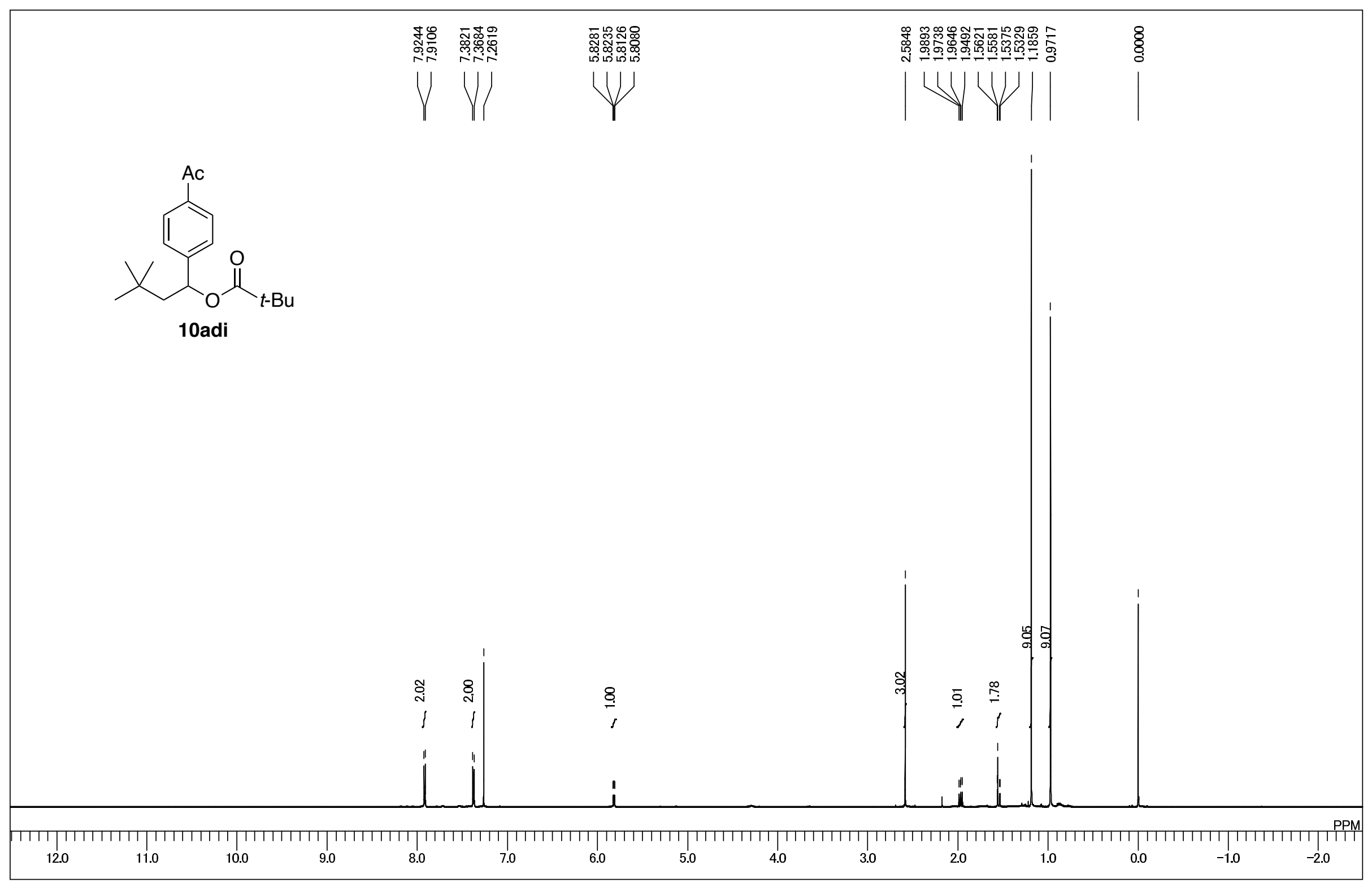




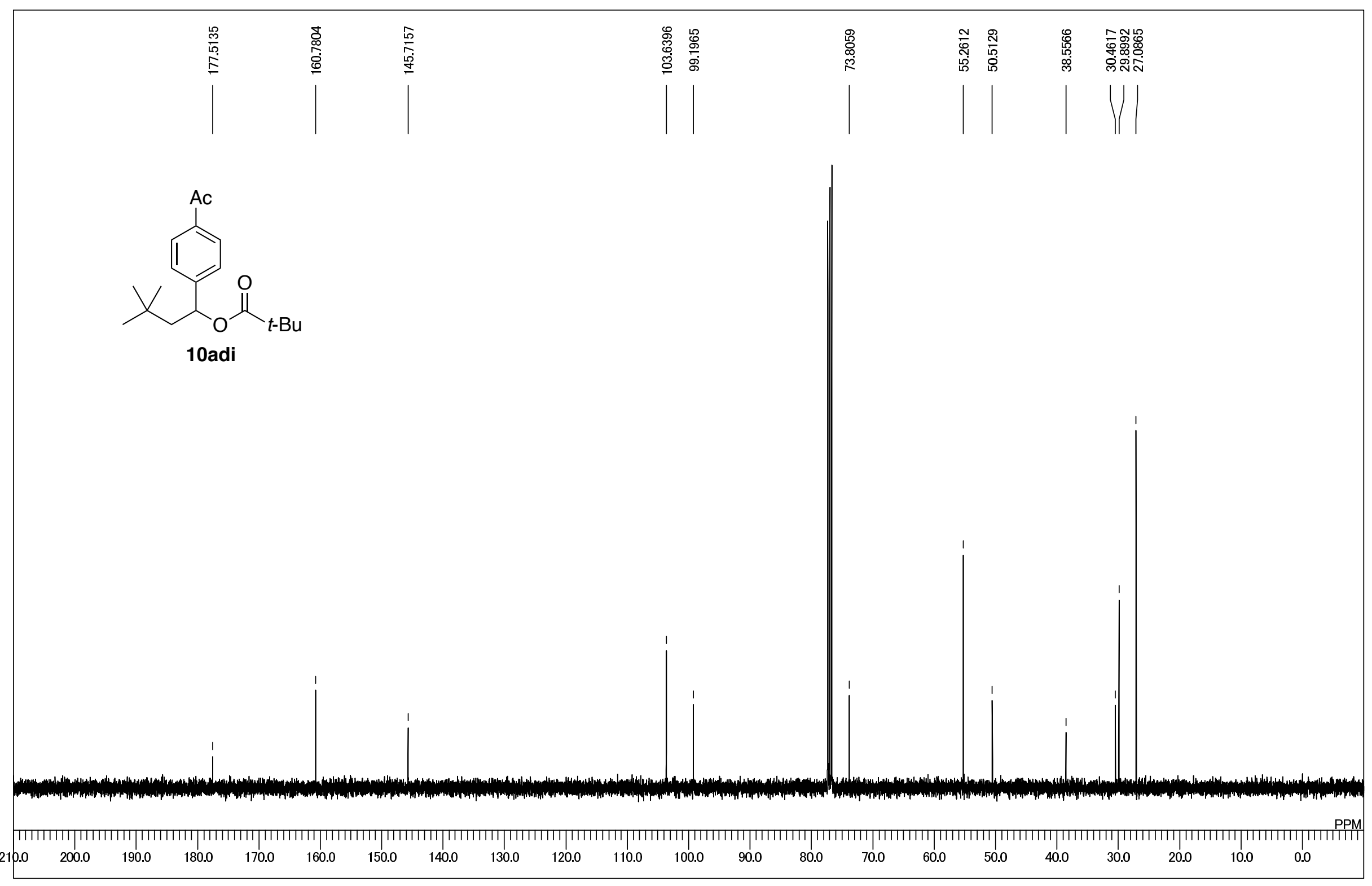




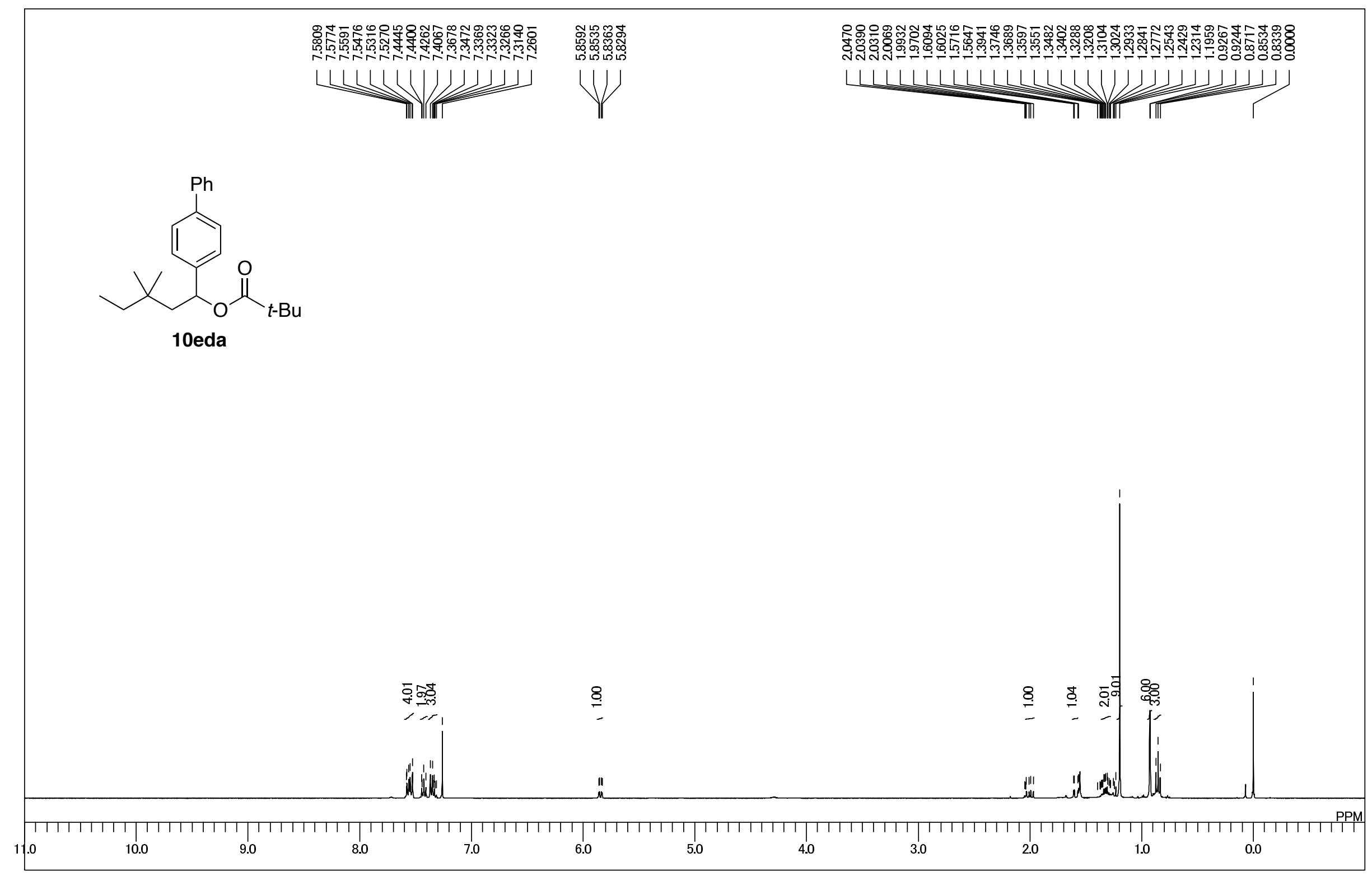




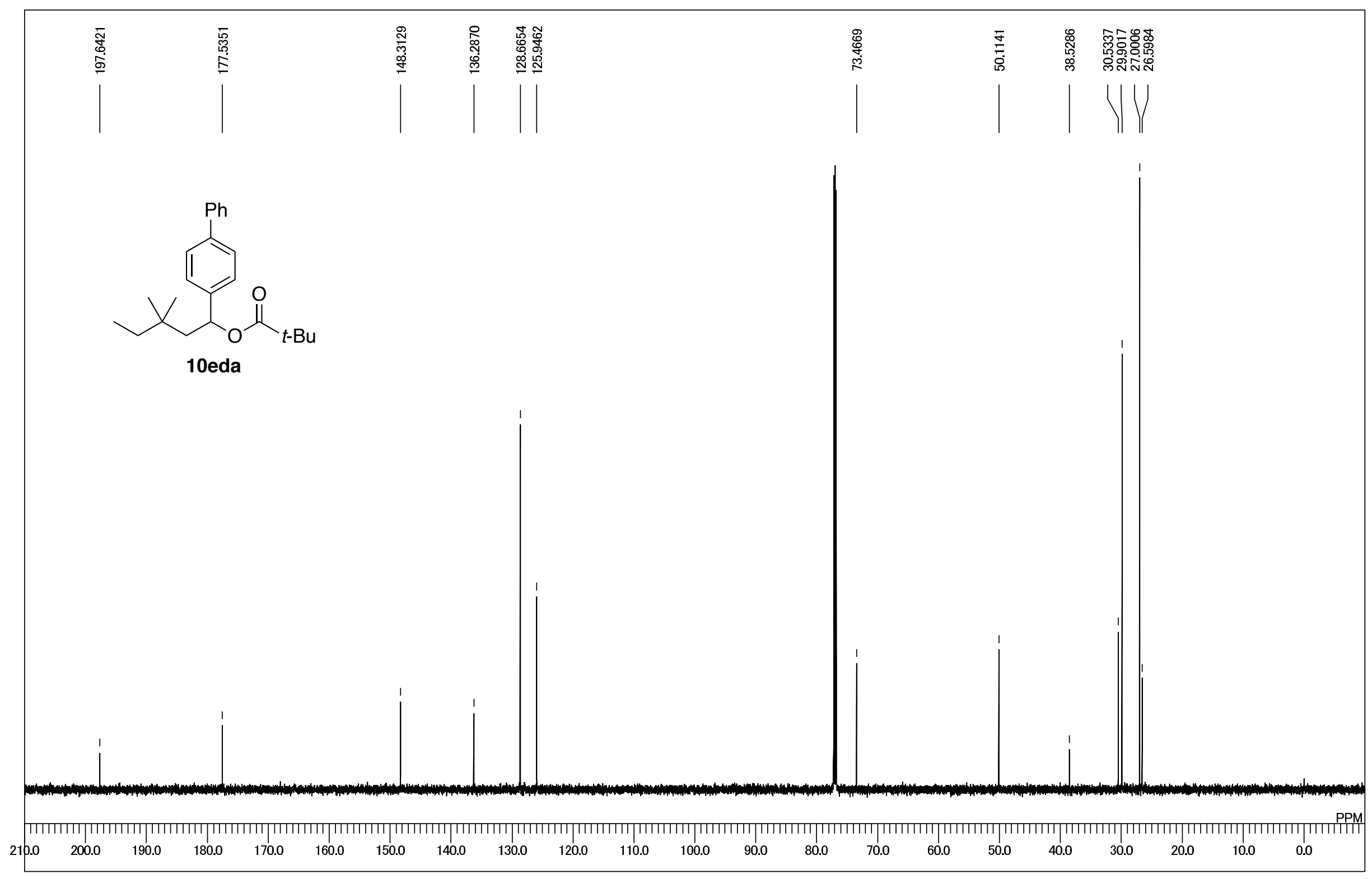

